\title{
Mind the gap : experimental studies on splanchnic hypoperfusion and gastrointestinal integrity loss in man
}

Citation for published version (APA):

van Wijck, K. (2013). Mind the gap : experimental studies on splanchnic hypoperfusion and gastrointestinal integrity loss in man. [Doctoral Thesis, Maastricht University]. Maastricht University. https://doi.org/10.26481/dis.20130704kw

Document status and date:

Published: 01/01/2013

DOI:

10.26481/dis.20130704kw

Document Version:

Publisher's PDF, also known as Version of record

Please check the document version of this publication:

- A submitted manuscript is the version of the article upon submission and before peer-review. There can be important differences between the submitted version and the official published version of record. People interested in the research are advised to contact the author for the final version of the publication, or visit the DOI to the publisher's website.

- The final author version and the galley proof are versions of the publication after peer review.

- The final published version features the final layout of the paper including the volume, issue and page numbers.

Link to publication

\footnotetext{
General rights rights.

- You may freely distribute the URL identifying the publication in the public portal. please follow below link for the End User Agreement:

www.umlib.nl/taverne-license

Take down policy

If you believe that this document breaches copyright please contact us at:

repository@maastrichtuniversity.nl

providing details and we will investigate your claim.
}

Copyright and moral rights for the publications made accessible in the public portal are retained by the authors and/or other copyright owners and it is a condition of accessing publications that users recognise and abide by the legal requirements associated with these

- Users may download and print one copy of any publication from the public portal for the purpose of private study or research.

- You may not further distribute the material or use it for any profit-making activity or commercial gain

If the publication is distributed under the terms of Article $25 \mathrm{fa}$ of the Dutch Copyright Act, indicated by the "Taverne" license above, 


\section{Mind the gap}

Experimental studies on splanchnic hypoperfusion and gastrointestinal integrity loss in man

Kim van Wijck 
(C) copyright Kim van Wijck, Maastricht 2013

Website: www.kimvanwijck.nl

The studies presented in this thesis were performed within the framework of Top Institute Food and Nutrition (TIFN). The studies were performed at the Maastricht University Medical Center+ in collaboration with the Nutrition and Toxicology Research Institute Maastricht (NUTRIM).

Layout: DTP-Studio-Aelberts en Kim vanWijck 


\section{Mind the gap}

Experimental studies on splanchnic hypoperfusion and gastrointestinal integrity loss in man

\section{DISSERTATION}

to obtain the degree of Doctor at the Maastricht University, on the authority of the Prof. dr. L.L.G. Soete, Rector Magnificus, in accordance with the decision of the Board of Deans, to be defended in public on Thursday 4th of July 2013, at 14:00 hours by

Kim van Wijck 


\section{PROMOTORES}

Prof. dr. W.A. Buurman

Prof. dr. C.H.C. Dejong

\section{COPROMOTOR}

Dr. K. Lenaerts

\section{BEOORDELINGSCOMMISSIE}

Prof. dr. P.B. Soeters, voorzitter

Prof. dr. S.D. Anker, Berlin, Germany

Prof. dr. A.P. de Bruïne

Prof. dr. J.J. Kolkman, Groningen

Prof. dr. W.H. Saris 


\section{Contents}

\section{Part I}

Chapter 1

Chapter 2

\section{Part II}

Chapter 3

Chapter 4

Chapter 5

Chapter 6

Part III

Chapter 7

Chapter 8

Chapter 9

Chapter 10

\section{Part IV}

Chapter 11

Chapter 12

Chapter 13

\section{Introduction}

General introduction $\quad 7$

Physiology and pathophysiology of splanchnic hypoperfusion and

intestinal injury during exercise: strategies for evaluation and prevention

\section{Loss of gastrointestinal integrity and permeability analysis}

Early diagnosis of intestinal ischemia using urinary and plasma fatty acid binding proteins

Polyethylene glycol versus dual sugar permeability assay:

is it time to choose?

Novel analytical approach to a multi-sugar whole gut permeability assay 79

Novel multi-sugar assay for site-specific gastrointestinal permeability

analysis

\section{Splanchnic hypoperfusion and loss of gastrointestinal integrity}

Exercise-induced splanchnic hypoperfusion results in gut dysfunction in healthy men

Dietary protein digestion and absorption are impaired during acute post-exercise recovery in young men

Aggravation of exercise-induced intestinal injury by ibuprofen in athletes

Oral citrulline supplementation prior to exercise improves

splanchnic perfusion and microcirculation during and after exercise in athletes

\section{Summary and discussion}

Discussion

English summary

Nederlandse samenvatting

Dankwoord

Curriculum vitae en publicaties

Scientific output

Grands and awards 



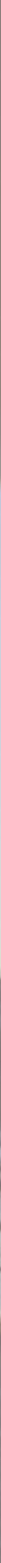

Chapter 1 
Part I - Chapter 1 


\section{INTRODUCTION AND OUTLINE OF THIS THESIS}

The gastrointestinal (GI) tract has two very important functions: (I) the absorption of nutrients and water, and (II) the maintenance of an effective barrier against harmful dietary antigens, digestive enzymes, and commensal and foreign bacterial pathogens and toxins. Many physiological and pathophysiological situations have been reported to compromise the GI system and to disrupt the GI epithelial barrier, posing a considerable threat to the human body. Examples of such situations are intestinal ischemia, celiac disease, pancreatitis, systemic inflammatory response syndrome, heavy physical exercise and the use of specific medication (non-steroid anti-inflammatory drugs, NSAIDs). The diagnosis of barrier integrity loss is hampered by the absence of reliable, non-invasive tests. Diagnostic delay interferes with patient recovery and impedes early therapeutic intervention, leading to higher morbidity and mortality rates.In this chapter, the anatomy and regulation of the GI epithelial barrier will be introduced, including a brief overview of situations associated with GI barrier dysfunction. Chapter 2 will elaborate on one of these situations, namely strenuous physical exercise, and discusses modalities to evaluate exercise-induced loss of GI integrity.

\section{Physical GI barrier}

Two key features are essential to maintain an effective barrier against luminal components: the first is the physical barrier of the GI epithelium, the second is an effective immune response. An uncompromised physical barrier is composed of a continuous epithelial monolayer of enterocytes, which are differentiated cells originating from stem cells present in the crypt. Furthermore, the integrity of the gut epithelial barrier is sustained by tight junctions, adherence junctions, gap junctions, and desmosomes $(33,34)$.

The intestinal epithelium undergoes rapid renewal. Stem cells in the crypt give rise to rapidly amplifying cells, which migrate upwards along the villus axis and differentiate into mature epithelial cells. Upon reaching the villus tip, the mature enterocytes die and are shed into the intestinal lumen following cell detachment, a process that constantly occurs in physiological situations. In addition, epithelial cells affected by infection or topical damage are rapidly exfoliated and replenished by neighboring cells. This epithelial cell turnover helps to expel pathogens and prevents pathological bacterial colonization, translocation, and inflammation $(10,31)$.

The tight junctions are major, highly dynamic complexes that are composed of many different proteins that are anchored in the cell via the actin cytoskeleton (20). The tight junction proteins are divided into intracellular proteins, the zonula occludens $(\mathrm{ZO})$ proteins, and transmembrane proteins such as junctional adhesion molecules (JAM), claudins, and occludins. These proteins tightly act together to form a dynamic fence against foreign antigens and microbes. Adherence junctions, gap junctions, and desmosomes all work together to form apical junctional complexes that interact with neighbouring cells to preserve epithelial architecture, facilitate cell migration during epithelial renewal, and enable intercellular communication $(11,17)$. 
The physical intestinal barrier is further reinforced by the presence of a continuous mucus layer that overlies the intestinal epithelium. The mucus of this layer is produced by goblet cells that are found scattered along the epithelium (32).

\section{Immunological GI barrier}

The main contributors to the immunological barrier within the GI system are the intestinal epithelial cells (IECs), including enterocytes and Paneth cells, and the myeloid and lymphoid cells of the intestinal tract. The IECs are members of the innate immune system, they produce antimicrobial proteins, and are able to sense potentially harmful bacterial products via pattern recognition receptors (PRRs). PRRs recognize specific pathogen-associated molecular patterns (PAMPs), including lipopolysaccharide present on the gram-negative bacterial wall and peptidoglycans mainly on the wall of gram-positive bacterial pathogens (26). If such PAMPs bind to their associated PRRs, signalling cascades are activated, leading to the initiation of an inflammatory response specific to the recognized microbial molecules (18). Paneth cells, located in the crypts of the intestine in between the stem cells, contribute to the immunological barrier of the small intestine by producing and secreting antimicrobial proteins into the intestinal lumen to prevent bacterial translocation and to protect the stem cells (4). Paneth cells also recognize PAMPs present in the lumen, leading to the production and secretion of $\alpha$-defensins (39). In addition, specialized myeloid and lymphoid structures embedded in the lamina propria of the small intestine contain cells that are capable of microbial and antigen sampling from the intestinal lumen (2). Subsequent delivery of the sampled material to the underlying lymphoid tissue results in the generation of an appropriate immune response with activation and recruitment of innate immune cells (2).

Another defense mechanism that prevents bacterial pathogens from breaching the epithelial lining is the presence of approximately $10^{14}$ commensal bacteria within the intestinal tract (3). These commensal bacteria exert an important role in maintaining GI barrier function $(9,19)$. Commensal bacteria stimulate epithelial cell turnover, compete with pathogenic bacteria, and contribute to the development and function of the immune system (3).

\section{Regulation of GI barrier function and GI permeability}

GI barrier function is regulated by a number of processes, including the high turnover of intestinal epithelial cells, the highly dynamic apical junctional complexes that seal the paracellular space in between the epithelial cells, and the interplay with the innate and adaptive immune response.

Intestinal epithelial cell damage leads to loss of cellular integrity, likely resulting in increased transcellular permeability for small solutes across the GI epithelial barrier (21). The junctional complexes between the epithelial cells largely regulate GI permeability. The tight junctions limit solute fluxes across the GI epithelium via the paracellular pathway, and are therefore considered the rate-limiting step in paracellular transepithelial transport (35). Data suggest the existence of at least two, probably independent, paracellular routes across the tight junction $(13,35,41)$. 
The flux of macromolecular solutes occurs via a size-selective 'leak' pathway, whereas small $(<4$ Å radius) solutes permeate through smaller, charge-selective pathways $(13,41)$. Both the sizeselective and charge-selective tight junction pathways are regulated by a variety of physiological and pathophysiological stimuli (35).

Studies have revealed a role for sodium $\left(\mathrm{Na}^{+}\right)$as a physiological regulator of the tight junction barrier $(36,37)$. Active uptake of $\mathrm{Na}^{+}$at the villus apex results in glucose co-transport and absorption of water and small solutes (34). This $\mathrm{Na}^{+}$-induced regulation of tight junction permeability occurs via activation of myosin light chain kinase (MLCK), which leads to phosphorylation of myosin light chain (MLC) in the actomyosin ring that surrounds the junctional complex and to opening of the tight junction, thereby increasing paracellular permeability $(35,37)$.

Immunological stimuli can also increase paracellular permeability via MLCK-induced changes in the tight junctional complex $(35,37)$. In addition, tumor necrosis factor $\alpha$ (TNF- $\alpha$ ), an important mediator of intestinal inflammation, was found to lead to degradation of occludin messenger RNA (mRNA), resulting in depletion of occludin, a transmembrane tight junction protein (43). The latter led to increased intestinal permeability and macromolecular flux via the 'leak' paracellular pathway $(1,43)$.

Another stimulus that has been described to induce structural changes in the tight junction complex is intestinal ischemia (16). After ischemia and reperfusion, changes in MLCK protein levels were suggestive of a phosphorylated MLC-induced epithelial constriction at sites where epithelial cells had lost contact with the basal membrane (16). After prolonged ischemia followed by reperfusion, profound epithelial barrier loss was observed, with loss of tight junction proteins and significant translocation of bacterial endotoxin (15).

In short, the GI epithelium is constantly exposed to luminal content, including antigens, digestive enzymes, and bacterial pathogens that produce harmful toxins. The highly dynamic GI epithelial barrier is tightly regulated to allow only selective transmucosal transport. Loss of GI barrier integrity results in the permeability of the potentially harmful luminal content, and may cause profound intestinal injury and inflammation.

\section{Loss of GI integrity in physiology and pathophysiology}

Loss of integrity occurs in many physiological and pathophysiological situations. The physiological situations associated with GI integrity loss that will be discussed in the current thesis include heavy physical exercise and experimentally induced GI integrity loss after 2-day oral intake of NSAID, both in healthy individuals.

During heavy physical exercise, redistribution of splanchnic blood flow reduces the circulation in the abdominal region, leading to splanchnic hypoperfusion (8). Such hypoperfusion or ischemia gives rise to intestinal mucosal injury due to a lack of oxygen and nutrients, depriving especially the mature enterocytes of resources necessary for survival, since these cells already are in a hypoxic state 
due to the countercurrent circulation of the intestinal villi (7). Injured enterocytes may be rapidly expelled into the GI lumen, after being detached from the basal membrane. While at this stage the epithelial lining of the intestine may be macroscopically intact, functional barrier integrity loss and increased GI permeability are already present $(24,28)$.

The second physiological situation associated with GI integrity loss studied was the intake of NSAIDs. NSAIDs are known to cause GI injury, especially after prolonged use $(6,23)$, but even short-term NSAID consumption was described to increase GI permeability $(6,44)$. These studies on physiological GI integrity loss aimed to clarify the origin and the extent of the epithelial compromise. Furthermore, the aim was to develop accurate measures of GI integrity loss.

Other, more severe situations that have been associated with loss of GI integrity in relatively healthy individuals include psychological stress (22), excessive consumption of alcoholic beverages (30), and oral intake of specific types of food (38). In addition, pathological loss of GI integrity has been described in a wide variety of diseases, including intestinal diseases such as celiac disease (40), inflammatory bowel disease (5), viral infections (27), as well as systemic diseases such as autoimmune disorders (12) and sepsis (14). While many of the phenomena contributing to GI barrier loss remain to be clarified, we consider that splanchnic hypoperfusion and inflammation both play an important role in the development of GI barrier integrity loss in physiological and pathophysiological situations.

Another severe manifestation of mucosal integrity loss is GI ischemia. Ischemia of the GI tract may be the result of an acute occlusion of the celiac artery or splanchnic arteries, or may be the result of a long-term progressive atherosclerotic process in the afferent vessels within the splanchnic area $(25,29,42)$.

\section{Outline of this thesis}

The current thesis deals with two main issues. First of all, we developed and evaluated tools to assess loss of GI integrity. These tools were subsequently used to improve our understanding of GI integrity loss in case of splanchnic hypoperfusion during strenuous physical exercise. Chapter 2 is a review of literature on the development and evaluation of splanchnic hypoperfusion during physical exercise. The role of physiological splanchnic hypoperfusion in the development of epithelial integrity loss and abdominal distress during physical exercise is discussed, an overview is given of the available tools to assess loss of epithelial integrity, and some promising preventive and therapeutic interventions are discussed. In chapter 3, the role of plasma markers intestinal fatty acid binding protein (I-FABP), ileal bile acid binding protein (I-BABP), and liver fatty acid binding protein (L-FABP) to assess intestinal injury in patients with acute intestinal ischemia is described. Chapter 4 describes the evaluation of two permeability tests to analyze loss of GI barrier function. The first test is based on the urinary excretion of three differently sized polyethylene glycol (PEG) probes, whereas the second test uses two differently sized sugars to evaluate small intestinal permeability. The aim of this study was to compare urinary probe excretion 
and accuracy of both tests in healthy volunteers with increased GI permeability after short-term use of NSAID. In chapter 5, the improved analytical work-up of a multi-sugar test to evaluate GI permeability in both urinary and plasma samples is reported. The aim of this study was to expand the application of the sugar-based permeability analysis by adding three sugars for sitespecific whole gut permeability assessment, and to enable permeability analysis in plasma samples. The study described in chapter 6 focusses on the validation of the multi-sugar permeability assay described in chapter 5 , in healthy volunteers in a randomized crossover study. Urinary probe excretion and accuracy of the permeability analysis obtained using the multi-sugar assay were compared with data obtained using the dual sugar lactulose-rhamnose test.

The second part of this thesis elaborates on splanchnic hypoperfusion and its consequences on the GI system, including loss of GI integrity in a physiological situation. The study described in chapter 7 was designed to clarify the effects of splanchnic hypoperfusion during strenuous exercise on the gut, and the relationship between hypoperfusion and loss of GI integrity. In chapter $\mathbf{8}$, the effect of heavy exercise on epithelial integrity and protein uptake in young healthy volunteers is described. Chapter 9 describes a study on the potential role of citrulline to improve splanchnic perfusion and diminish exercise-induced epithelial injury in healthy, young volunteers. The effect of citrulline on exercise-induced changes in splanchnic perfusion and microcirculation were determined using gastric exercise tonometry and sublingual sidestream dark field imaging, respectively. In chapter 10, the effect of NSAIDs on GI epithelial integrity loss in rest and during exercise was studied using both plasma I-FABP levels and sugar-based GI permeability analysis.

Chapter 11 discusses the main issues of this thesis, some of the questions that were raised during the construction of this thesis, and interesting points for future studies are discussed. Chapter 12 provides a brief English and a brief Dutch overview of the main issues that were addressed in this thesis. 


\section{REFERENCES}

1. Al-Sadi R, Khatib K, Guo S, Ye D, Youssef M, and Ma T. Occludin regulates macromolecule flux across the intestinal epithelial tight junction barrier. A $m$ F Physiol Gastrointest Liver Physiol 300: G1054-1064, 2011.

2. Artis D. Epithelial-cell recognition of commensal bacteria and maintenance of immune homeostasis in the gut. Nat Rev Immunol 8: 411-420, 2008.

3. Ashida H, Ogawa M, Kim M, Mimuro H, and Sasakawa C. Bacteria and host interactions in the Gut epithelial barrier. Nat Chem Biol 8: 36-45, 2012.

4. Ayabe T, Satchell DP, Wilson CL, Parks WC, Selsted ME, and Ouellette AJ. Secretion of microbicidal $\alpha$-defensins by intestinal Paneth cells in response to bacteria. Nat Immunol 1: 113-118, 2000.

5. Bjarnason I, O'Morain C, Levi AJ, and Peters TJ. Absorption of 51 chromium-labeled ethylenediaminetetraacetate in inflammatory bowel disease. Gastroenterology 85: 318-322, 1983.

6. Bjarnason I, and Takeuchi K. Intestinal permeability in the pathogenesis of NSAID-induced enteropathy. $\mathcal{F}$ Gastroenterol 44 Suppl 19: 23-29, 2009.

7. Blikslager AT. Life in the Gut without oxygen: adaptive mechanisms and inflammatory bowel disease. Gastroenterology 134: 346-348, 2008.

8. Bradley SE, Childs AW, Combes B, Cournand A, Wade OL, and Wheeler HO. The effect of exercise on the splanchnic blood flow and splanchnic blood volume in normal man. Clin Sci (Lond) 15: 457-463, 1956.

9. Cario E, Gerken G, and Podolsky DK. Toll-like receptor 2 enhances ZO-1-associated intestinal epithelial barrier integrity via protein kinase C. Gastroenterology 127: 224-238, 2004.

10. Chichlowski M, and Hale LP. Bacterial-mucosal interactions in inflammatory bowel disease: an alliance gone bad. Am 7 Physiol Gastrointest Liver Physiol 295: G1139-1149, 2008.

11. Ey B, Eyking A, Gerken G, Podolsky DK, and Cario E. TLR2 mediates gap junctional intercellular communication through connexin-43 in intestinal epithelial barrier injury. F Biol Chem 284: 22332-22343, 2009.

12. Fasano A. Zonulin and its regulation of intestinal barrier function: the biological door to inflammation, autoimmunity, and cancer. Physiological reviews 91: 151-175, 2011.

13. Fihn BM, Sjoqvist A, and Jodal M. Permeability of the rat small intestinal epithelium along the villus-crypt axis: effects of glucose transport. Gastroenterology 119: 1029-1036, 2000.

14. Fink MP. Gastrointestinal mucosal injury in experimental models of shock, trauma, and sepsis. Critical care medicine 19: 627-641, 1991.

15. Grootjans J, Lenaerts K, Derikx JP, Matthijsen RA, de Bruine AP, van Bijnen AA, van Dam RM, Dejong CH, and Buurman WA. Human intestinal ischemia-reperfusion-induced inflammation characterized: experiences from a new translational model. The American journal of pathology 176: 2283-2291, 2010.

16. Grootjans J, Thuijls G, Derikx JP, van Dam RM, Dejong CH, and Buurman WA. Rapid lamina propria retraction and zipper-like constriction of the epithelium preserves the epithelial lining in human small intestine exposed to ischaemia-reperfusion. $\mathcal{F}$ Pathol 224: 411-419, 2011.

17. Harris TJ, and Tepass U. Adherens junctions: from molecules to morphogenesis. Nat Rev Mol Cell Biol 11: 502-514, 2010 .

18. Hisamatsu T, Suzuki M, Reinecker HC, Nadeau WJ, McCormick BA, and Podolsky DK. CARD15/NOD2 functions as an antibacterial factor in human intestinal epithelial cells. Gastroenterology 124: 993-1000, 2003.

19. Hooper LV, and Gordon JI. Commensal host-bacterial relationships in the gut. Science 292: 1115-1118, 2001.

20. Ivanov AI, McCall IC, Parkos CA, and Nusrat A. Role for actin filament turnover and a myosin II motor in cytoskeleton-driven disassembly of the epithelial apical junctional complex. Molecular Biology of the Cell 15: 2639-2651, 2004.

21. John LJ, Fromm M, and Schulzke JD. Epithelial barriers in intestinal inflammation. Antioxid Redox Signal 15: 1255-1270, 2011.

22. Konturek PC, Brzozowski T, and Konturek SJ. Stress and the gut: pathophysiology, clinical consequences, diagnostic approach and treatment options. F Physiol Pharmacol 62: 591-599, 2011.

23. Maiden L, Thjodleifsson B, Seigal A, Bjarnason, II, Scott D, Birgisson S, and Bjarnason I. Long-term effects of nonsteroidal anti-inflammatory drugs and cyclooxygenase- 2 selective agents on the small bowel: a cross-sectional capsule enteroscopy study. Clin Gastroenterol Hepatol 5: 1040-1045, 2007.

24. Marchbank T, Davison G, Oakes JR, Ghatei MA, Patterson M, Moyer MP, and Playford RJ. The nutriceutical bovine colostrum truncates the increase in Gut permeability caused by heavy exercise in athletes. Am F Physiol Gastrointest Liver Physiol 300: G477-484, 2011.

25. Mensink PB, Moons LM, and Kuipers EJ. Chronic gastrointestinal ischaemia: shifting paradigms. Gut 60: $722-$ 737,2011

26. Meylan E, Tschopp J, and Karin M. Intracellular pattern recognition receptors in the host response. Nature 442: 39-44, 2006.

27. Noone C, Menzies IS, Banatvala JE, and Scopes JW. Intestinal permeability and lactose hydrolysis in human rotaviral gastroenteritis assessed simultaneously by non-invasive differential sugar permeation. European journal of clinical investigation 16: 217-225, 1986. 
28. Oktedalen O, Lunde OC, Opstad PK, Aabakken L, and Kvernebo K. Changes in the gastrointestinal mucosa after long-distance running. Scand F Gastroenterol 27: 270-274, 1992.

29. Oldenburg WA, Lau LL, Rodenberg TJ, Edmonds HJ, and Burger CD. Acute mesenteric ischemia: a clinical review. Archives of internal medicine 164: 1054-1062, 2004.

30. Purohit V, Bode JC, Bode C, Brenner DA, Choudhry MA, Hamilton F, Kang YJ, Keshavarzian A, Rao R, Sartor RB, Swanson C, and Turner JR. Alcohol, intestinal bacterial growth, intestinal permeability to endotoxin, and medical consequences: summary of a symposium. Alcohol 42: 349-361, 2008.

31. Radtke F, and Clevers H. Self-renewal and cancer of the gut: two sides of a coin. Science 307: 1904-1909, 2005.

32. Sansonetti PJ. War and peace at mucosal surfaces. Nat Rev Immunol 4: 953-964, 2004.

33. Schwarz BT, Wang F, Shen L, Clayburgh DR, Su L, Wang Y, Fu YX, and Turner JR. LIGHT signals directly to intestinal epithelia to cause barrier dysfunction via cytoskeletal and endocytic mechanisms. Gastroenterology 132: 2383-2394, 2007.

34. Shen L, Weber CR, Raleigh DR, Yu D, and Turner JR. Tight junction pore and leak pathways: a dynamic duo. Annu Rev Physiol 73: 283-309, 2011.

35. Turner JR. Intestinal mucosal barrier function in health and disease. Nature Rev Immunol 9: 799-809, 2009.

36. Turner JR, and Madara JL. Physiological regulation of intestinal epithelial tight junctions as a consequence of $\mathrm{Na}(+)$-coupled nutrient transport. Gastroenterology 109: 1391-1396, 1995.

37. Turner JR, Rill BK, Carlson SL, Carnes D, Kerner R, Mrsny RJ, and Madara JL. Physiological regulation of epithelial tight junctions is associated with myosin light-chain phosphorylation. Am f Physiol 273: C1378-1385, 1997.

38. Ulluwishewa D, Anderson RC, McNabb WC, Moughan PJ, Wells JM, and Roy NC. Regulation of tight junction permeability by intestinal bacteria and dietary components. F Nutr 141: 769-776, 2011.

39. Vaishnava S, Behrendt CL, Ismail AS, Eckmann L, and Hooper LV. Paneth cells directly sense Gut commensals and maintain homeostasis at the intestinal host-microbial interface. Proc Natl Acad Sci U S A 105: 20858-20863, 2008.

40. van Elburg RM, Uil JJ, Mulder CJ, and Heymans HS. Intestinal permeability in patients with coeliac disease and relatives of patients with coeliac disease. Gut 34: 354-357, 1993.

41. Van Itallie CM, Holmes J, Bridges A, Gookin JL, Coccaro MR, Proctor W, Colegio OR, and Anderson JM. The density of small tight junction pores varies among cell types and is increased by expression of claudin-2. $\mathcal{F}$ Cell Sci 121: 298-305, 2008.

42.van Noord D, Sana A, Moons LM, Pattynama PM, Verhagen HJ, Kuipers EJ, and Mensink PB. Combining radiological imaging and gastrointestinal tonometry: a minimal invasive and useful approach for the workup of chronic gastrointestinal ischemia. European journal of gastroenterology $\mathcal{E}$ hepatology 2013.

43. Ye D, Guo S, Al-Sadi R, and Ma TY. MicroRNA regulation of intestinal epithelial tight junction permeability. Gastroenterology 141: 1323-1333, 2011.

44. Zeino Z, Sisson G, and Bjarnason I. Adverse effects of drugs on small intestine and colon. Best Pract Res Clin Gastroenterol 24: 133-141, 2010. 


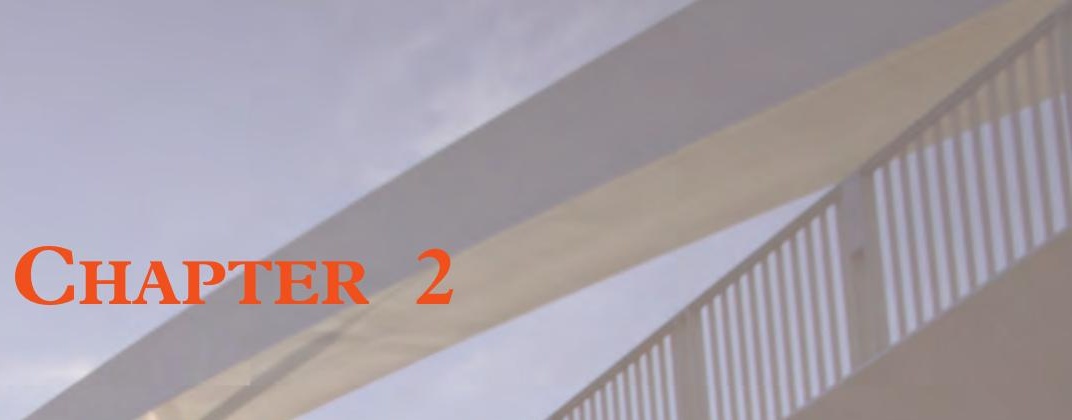

Physiology and pathophysiology of splanchnic hypoperfusion and intestinal injury during exercise: strategies for evaluation and prevention
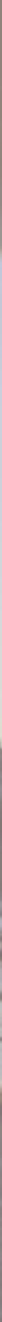


\section{ABSTRACT}

Physical exercise places high demands on the adaptive capacity of the human body. Strenuous physical performance increases the blood supply to active muscles, cardiopulmonary system, and skin to meet the altered demands for oxygen and nutrients. The redistribution of blood flow, necessary for such an increased blood supply to the periphery, significantly reduces blood flow to the gut, leading to splanchnic hypoperfusion and gastrointestinal (GI) compromise. A compromised GI system can have a negative impact on exercise performance and subsequent post-exercise recovery due to abdominal distress and impairments in the uptake of fluid, electrolytes, and nutrients. In addition, strenuous physical exercise leads to loss of epithelial integrity, which may give rise to increased intestinal permeability with bacterial translocation and inflammation. Ultimately, these effects can deteriorate post-exercise recovery and disrupt exercise training routine.

This review provides an overview on the recent advances in our understanding of GI physiology and pathophysiology in relation to strenuous exercise. Various approaches to determine the impact of exercise on the individual athlete's GI tract will be discussed. In addition, we will elaborate on several promising components that could be exploited for preventive interventions. 


\section{INTRODUCTION}

The gastrointestinal (GI) system plays an important role in the athlete's ability to perform strenuous exercise (24). Proper GI function enables endurance performance and promotes early recovery. In contrast, a compromised GI system will likely impair performance and subsequent recovery, and may give rise to serious GI problems, such as nausea, vomiting, angine abdominale and bloody diarrhea $(102,125)$. Such symptoms, manifestations of a compromised GI system, are experienced by 25 to $70 \%$ of endurance athletes $(102,125)$. Among the reported deleterious manifestations of strenuous exercise are mucosal erosions and ischemic colitis, both observed after long distance running $(4,21,25,54,89,95)$. While the etiology of exercise-induced GI distress is multifactorial, GI ischemia is often acknowledged as the main pathophysiological mechanism for the emergence of such distress $(29,89,124,125)$.

This review provides an overview on the current understanding of GI physiology and pathophysiology during and after exercise. Strategies will be discussed that may be used by athletes to determine the impact of exercise on the GI tract. Ultimately, we will provide insight into some promising resources that may be used to prevent exercise-induced abdominal distress.

\section{Gastrointestinal perfusion}

The arterial blood supply of the GI tract consists of three main afferent routes: the celiac trunk, and the superior and inferior mesenteric artery (81). These three main arteries ramify into three dense vascular plexus, the serosal, submucosal and mucosal plexus, of which the latter shoots the microcirculatory capillary network that irrigates the intestinal mucosa. Drainage of the splanchnic area is covered by the superior mesenteric vein, inferior mesenteric vein, and the portal vein that return the venous blood to the heart via the liver and subsequently the inferior vena cava (81). The splanchnic vasculature constitutes a system with a remarkable capacity to adapt to physiological stressors promoting vasodilatation or constriction via regulation of the mesenteric vascular resistance by neuroendocrine, humoral, and paracrine mechanisms (79). During strenuous physical activity or exercise, the release of norepinephrine near the $\alpha$-adrenoreceptors of the sympathetic nervous system induces splanchnic vasoconstriction, thereby increasing total splanchnic vascular resistance $(41,81,141)$. The blood is rapidly redistributed from the splanchnic area to be used for the perfusion of tissues with increased activity during exercise, such as heart, lungs, active muscle and skin (Figure 1) $(98,109)$.

Although Doppler ultrasound and contrast extraction studies in the nineteen fifties had already revealed a vast reduction in hepatosplanchnic blood flow during exercise $(14,111)$, it was the technique of gastric tonometry that revealed that strenuous physical exercise results in splanchnic hypoperfusion and mucosal acidosis in healthy individuals $(92,98)$. Tonometry is a functional 


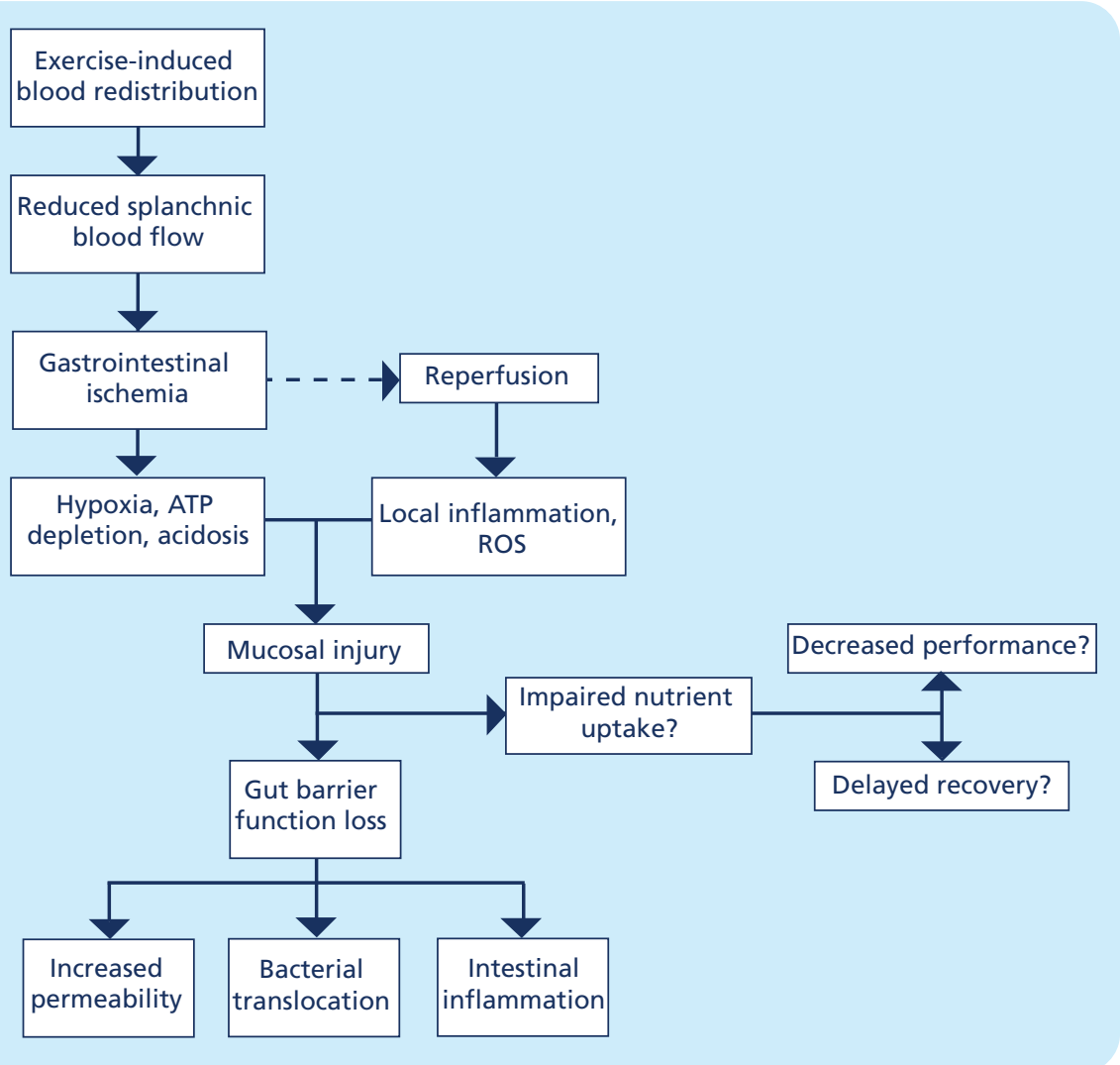

Figure 1: Schematic overview of the processes proposed to play a role in the development of exercise-induced gastrointestinal injury

Strenuous physical exercise leads to redistribution of blood, shunting blood away from the splanchnic area, thereby significantly reducing splanchnic blood flow. The ensuing ischemia leads to tissue hypoxia, local depletion of adenosinetriphosphate (ATP), and acidosis. After exercise, when splanchnic circulation is restored, reperfusion injury may develop, characterized by local inflammation and formation of reactive oxygen species (ROS). Both ischemia and reperfusion sequelae lead to mucosal damage and Gut wall integrity loss, which is accompanied by increased permeability, bacterial translocation and intestinal inflammation.

It remains to be clarified whether the exercise-induced mucosal injury impairs nutrient uptake, potentially decreasing athletic performance and delaying post-exercise recovery.

test that relies on the principle that inadequate tissue perfusion and tissue hypoxia lead to mucosal accumulation of carbon dioxide $\left(\mathrm{CO}_{2}\right)$. The exact working mechanism of gastric tonometry during exercise is depicted in Figure 2. In short, the accumulated $\mathrm{CO}_{2}$ increases the gastric $\mathrm{CO}_{2}$ tonus $\left(\mathrm{pCO}_{2}\right)$, which is measured by the tonometer (67), and the gap between gastric and systemic $\mathrm{pCO}_{2}$ reflects the adequacy of splanchnic perfusion. Gastric exercise tonometry revealed a rapid onset of splanchnic hypoperfusion, with the most pronounced changes in splanchnic perfusion occurring in the first ten minutes of strenuous exercise (135) (Figure 3A), indicating a rapid response of the splanchnic vascular bed. In addition, a rapid restoration of splanchnic 


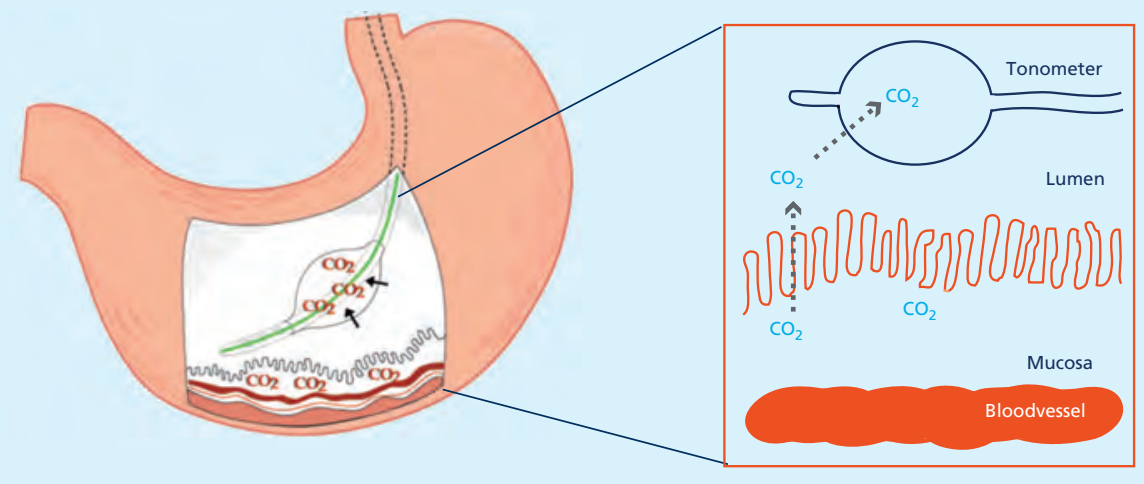

Figure 2: Working mechanism of gastric tonometry

Gastric tonometry relies on the principle that inadequate perfusion and tissue hypoxia lead to mucosal accumulation of $\mathrm{CO}_{2}$ due to anaerobic metabolism and decreased transport of $\mathrm{CO}_{2}$ via local blood vessels. The accumulated $\mathrm{CO}_{2}$ diffuses into the stomach, where it increases the $\mathrm{CO}_{2}$ tonus $\left(\mathrm{pCO}_{2}\right)$, which is detected via the balloon of the tonometry catheter connected to an extracorporeal tonometer. For accurate interpretation of the tonometry data, adequate suppression of gastric acid production is necessary to prevent buffering of the $\mathrm{CO}_{2}$ by $\mathrm{H}^{+}$, and arterial or arterialized blood samples should be collected to enable determination of the gap $\mathrm{pCO}_{2}$ (i.e. gastric-arterial $\mathrm{pCO}_{2}$ ). Arterialised blood samples can be obtained from an intravenous catheter inserted in a dorsal hand vein of a hand placed in a hot box in which the air is heated to $\sim 60^{\circ}$ Celsius.

Adjusted (with permission) from: Ackland et al, Critical Care 2000.

blood flow was observed in the first ten minutes of post-exercise recovery, suggesting rapid reperfusion of the splanchnic area (135) (Figure 1 and $3 A$ ).

Tonometry also demonstrated that the severity of splanchnic hypoperfusion was proportional to exercise intensity as assessed by increasing blood lactate levels (124), without a critical reduction in splanchnic perfusion during submaximal exercise in healthy individuals $(98,124)$. The majority of symptomatic athletes however, already developed splanchnic hypoperfusion during submaximal exercise (124), suggesting vascular hyperresponsiveness to exercise. In addition, early studies have pointed out that there is a clear relationship between the reduction in splanchnic blood flow and relative workload (23), suggesting that regular training has an impact on the sensitivity of the splanchnic vascular responsiveness to exercise. However, data comparing trained and matched untrained subjects are required to verify whether this is true.

Age has also been suggested to affect blood flow redistribution during exercise, since the vascular system in elderly may be less responsive to catecholamines (68), while atherosclerotic plaques and vascular stenosis associated with aging of the vascular system may also play a role. Reductions in splanchnic blood flow were indeed reported to be less pronounced in elderly (mean age 64 years) compared with their younger counterparts, who were closely matched with respect to maximal oxygen uptake capacity, when exercise intensities exceeded $60 \%$ of peak oxygen consumption (65). These observations corroborate the higher frequency of exercise-induced abdominal complaints in young athletes that may be hypoperfusion-related $(41,125)$, although the latter may of 


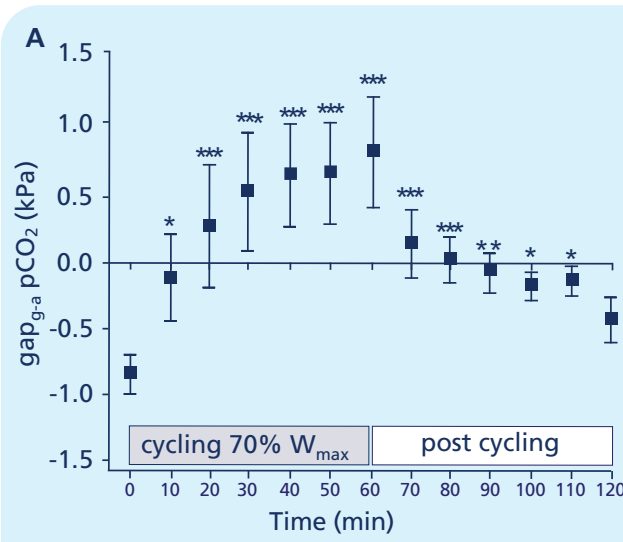

B

C
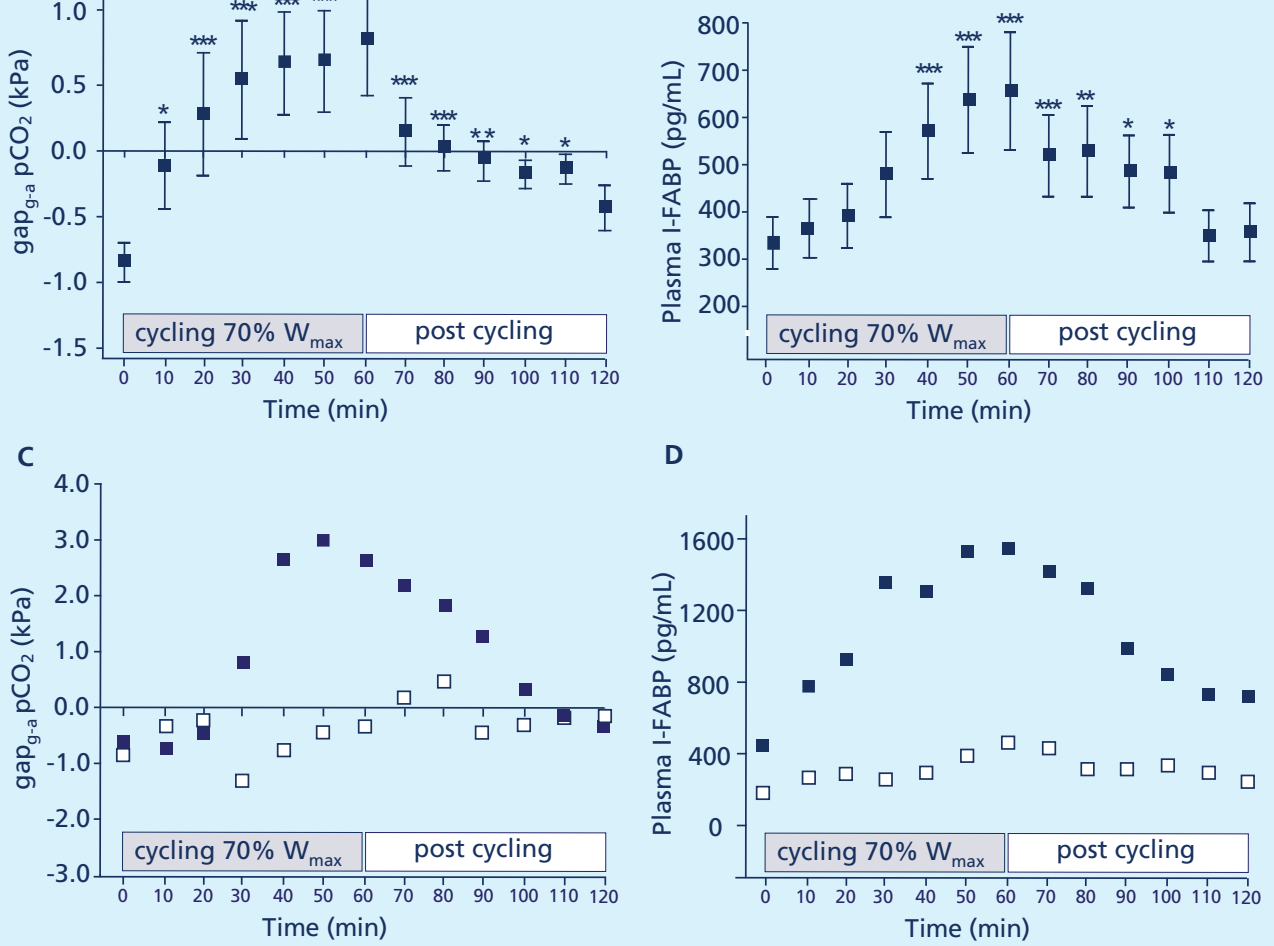

D

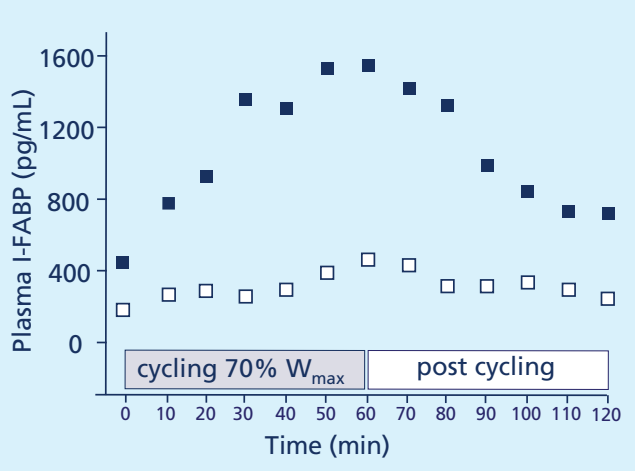

匹 individual A $\square$ individual B

Figure 3: Physical exercise results in splanchnic hypoperfusion and intestinal cell damage.

A) Gastric tonometry shows decreased splanchnic perfusion during and after cycling. B) Plasma I-FABP levels reflect the development of intestinal epithelial cell damage during and after cycling. C) Gastric tonometry shows two different splanchnic profiles of two individuals during and after exercise. D) Plasma I-FABP levels of the same two individuals reveal intestinal cell damage individual A (closed symbols), while individual B (open symbols) has no apparent intestinal damage during exercise, reflected by maintenance of baseline I-FABP levels. Data in A and $B$ represent mean and SEM. Different from baseline $(t=0)\left({ }^{\star} \mathrm{p}<0.01,{ }^{\star \star} \mathrm{p}<0.001,{ }^{\star \star \star} \mathrm{p}<0.0001\right)$. Adjusted from: Van Wijck et al, PLoS 2011

course also be related to the fact that in general, absolute workloads of young athletes are substantially higher than for the elderly, leading to more profound circulatory changes. Another explanation for the less extensive splanchnic hypoperfusion observed in the elderly is their reduced muscle mass.

In conclusion, exercise leads to redistribution of blood away from the splanchnic area, resulting in intestinal hypoperfusion and rapid reperfusion, which may contribute to GI distress in symptomatic athletes. 


\section{GI function: exercise-induced loss of epithelial integrity and barrier function}

Decreased oxygenation of the intestinal epithelium may lead to damage at the intestinal villus tips (Figure 1) (30, 49). In line, exercise-induced hypoperfusion leads to loss of cellular integrity in the intestine of young, healthy individuals during strenuous exercise, which is reflected by significantly increased plasma levels of intestinal fatty acid binding protein (I-FABP) (Figure 3B) (135). I-FABP is a small, cytosolic protein present in mature enterocytes at the upper half of the small intestinal villi, and is rapidly released into the circulation upon cellular injury (30, $101,128)$. The susceptibility of the mature enterocyte population to low-flow states and ischemic events is explained by the counter-current exchange mechanism of the villus $(10,11)$ that creates a constant low oxygen, hyperosmolar environment at the villus tip (51).

Reduced splanchnic perfusion deteriorates the physiological low oxygen state, thereby further depriving the enterocytes from oxygen and denosinetriphosphate (ATP), leading to epithelial cell damage, and loss of epithelial integrity (Figure 1) (30,94). In line, profound intestinal damage presenting as mucosal erosions with GI bleeding was observed during endoscopy in athletes after strenuous endurance running $(21,95)$. GI bleeding was also reported by other authors, after obtaining positive faecal occult blood tests (FOBT) from otherwise healthy athletes after long-distance running $(8,90,133)$. Ultimately, profound intestinal bleeding can lead to exercise-induced haemorrhagic colitis requiring surgery, a condition particularly described in marathon runners $(107,116)$.

Exercise-induced intestinal injury may be primarily the result of hypoperfusion or the result of a combination of ischemia with subsequent reperfusion, since restoration of the splanchnic blood flow can further compromise epithelial integrity due to oxidative stress and inflammation (Figure 1) $(36,49)$. Loss of epithelial integrity is associated with increased GI permeability, bacterial translocation, and intestinal inflammation (Figure 1) $(7,36)$. Such barrier integrity loss includes disruption of the tight junctions interconnecting the intestinal epithelial cells, either by alteration of the epithelial cytoskeleton or by loss of tight junction proteins $(59,126,127)$. Increased permeability and bacterial translocation may occur (7), and tight junction proteins are rapidly redistributed to quickly restore an effective intestinal barrier (77).

Increased intestinal permeability after strenuous exercise was confirmed by increased urinary excretion of orally ingested permeability probes such as ${ }^{51} \mathrm{Chromium}$ ethylene diamine tetraacetic acid $\left({ }^{51} \mathrm{Cr}\right.$ EDTA) (95) and sugar permeability probes $(76,99)$. Sugar-based permeability analysis relies on the permeation of large and small sugar probes through GI mucosa, which provides a specific index of GI permeability (9). An increase in this permeability index was also observed in plasma samples of young athletes, reflecting a transient increase in GI permeability after 1 hour of vigorous cycling, indicating disruption of the GI barrier (135). While healthy individuals are well able to cope with a transient increase in GI permeability, in case of prolonged, vigorous exercise, more profound increases in intestinal permeability lead to translocation of luminal endotoxins, resulting in endotoxaemia $(13,64)$. 
Another important line of defense in the small intestine that is worth mentioning in the setting of intestinal ischemia is the immunological barrier formed by the Paneth cells. These cells, located in the crypts of the intestine in between the stem cells, continuously produce and secrete antimicrobial proteins into the intestinal lumen to prevent bacterial translocation. In addition, they directly sense and respond to bacterial threats $(1,130)$. Recent studies have shown that intestinal ischemia-reperfusion in the human intestine is associated with Paneth cell apoptosis, and loss of these cells was indeed associated with increased bacterial translocation and inflammation (48). Although never investigated in the setting of physical activity or exercise, it is tempting to speculate on the involvement of Paneth cell dysfunction in the intestine of endurance athletes. During prolonged, vigorous exercise, splanchnic hypoperfusion may induce Paneth cell dysfunction, thereby possibly contributing to intestinal barrier function loss.

Loss of epithelial integrity, increased GI permeability and bacterial translocation induce a strong inflammatory response, which is characterized by complement activation, production and release of cytokines, endothelial activation and local neutrophil influx in the intestinal mucosa (30). Indeed, circulating levels of mannose-binding lectin (MBL), a player of innate immunity and an important initiating complement component (31), increased after 1 hour of vigorous cycling in healthy, young athletes (unpublished data), suggesting activation of the complement system. Furthermore, exercise led to elevated cytokine levels, and an increase in endothelium-derived factors and acute phase proteins $(17,100)$. While these inflammatory markers may originate from skeletal muscle (17, 100), increased faecal levels of neutrophil-derived calprotectin and bacterial translocation suggest an additional role for the intestinal mucosa as a contributor to the inflammatory response after strenuous exercise $(13,64,69,135)$.

In short, splanchnic hypoperfusion during exercise leads to loss of intestinal epithelial integrity, which may be accompanied by intestinal blood loss. Additionally, strenuous exercise results in loss of intestinal barrier integrity, reflected by increased permeability, bacterial translocation and intestinal inflammation (Figure 1).

\section{GI function: exercise-induced changes in digestion and absorption}

The consequences of vigorous exercise on GI function are diverse and many aspects remain to be clarified. For example, the effect of exercise on gastric emptying is very heterogeneous. While exercise at the individual's maximum capacity has been reported to delay gastric emptying in some studies $(16,38)$, the majority of studies fail to report any major changes in gastric emptying, even during more vigorous physical exercise $(43,121,132)$. Based on the current literature, it seems fair to conclude that gastric emptying is not limiting the speed of nutritional uptake during exercise when appropriate measures are taken regarding the volume, composition, temperature, and osmolality of the ingested drinks $(42,110,114)$. 
After gastric emptying, the luminal content enters the small intestine, the principal site of nutrient digestion and absorption. The latter is highly important with respect to digestive tolerance during exercise and determines the rate of nutrient supply to the active muscles (15). It is tempting to assume that GI function is reduced during vigorous physical exercise, and several authors have prompted that decreases in splanchnic perfusion and oxygen supply up to $80 \%$ reduce nutrient digestion and absorption $(15,29,132)$. In line, several studies have demonstrated that while glucose absorption is unaffected by exercise at low and moderate intensity levels, it is decreased during strenuous physical exercise $(71,132)$. Yet corroborative evidence supporting the theory that splanchnic hypoperfusion accounts for this decrease in absorption is still lacking. In addition to a putative effect of hypoperfusion on metabolic function, mucosal injury and local inflammation may exert negative metabolic effects during exercise. On the other hand, specific nutritional strategies may be able to preserve sufficient splanchnic flow during and after exercise. The latter contributes to the maintenance of a positive energy balance necessary to ensure optimal metabolic recovery and to promote optimal muscular regeneration processes (120).

Other factors that may contribute to decreased GI metabolic function are altered neurohormonal levels, altered secretory activities and disturbances in the electrolyte and acid-base balance (91). Last of all, inadequate fluid intake during strenuous exercise in a hot environment, leading to hypohydration of $3 \%$ of body weight did not interfere with digestion and absorption (112), but may impair intestinal barrier maintenance $(69,70)$.

In short, based on the available data, we consider that if strenuous exercise is prolonged, the need for energy substitution increases, while the absorptive capacity of the gut may decrease.

\section{Individual predisposition}

There is large heterogeneity in the response of the GI system to exercise. Splanchnic hypoperfusion during exercise ranges from mild circulatory changes to profound GI ischemia (Figure $3 A$ and $3 C$ ). In line, the consequences of hypoperfusion within the GI tract, i.e. epithelial injury and changes in GI permeability and epithelial barrier function, greatly differ between individuals (Figure $3 B$ and 3D). Ultimately, the presence and nature of abdominal symptoms experienced by athletes vary from mild, exercise-related discomfort to severe ischemic colitis and diarrhoea (88). A number of factors contributing to the pathophysiology of exercise-related abdominal distress have been identified, such as age, female sex, medication, dehydration, food intake, a hot, humid environment, type of exercise, exercise duration and exercise intensity $(29,41,102$, 125). Another factor that may contribute to the differences in the hypoperfusion sequelae is genetic background; e.g. previously, carriers of a homozygous MBL2 genotype were reported to have significantly lower levels of plasma I-FABP after intestinal ischemia (80), indicating that MBL-deficient individuals may be protected against ischemia/reperfusion-induced intestinal epithelial injury. An unfavourable combination of factors places the individual at risk of developing GI problems. 
Exercise-induced GI ischemia has been reported to occur in $50 \%$ of symptomatic athletes at submaximal exercise intensities, while asymptomatic athletes do not display intestinal ischemia during exercise at that level of intensity (124).

Importantly, specific underlying pathology may also give rise to GI problems during or shortly after physical exercise, and exercise-related symptoms may represent an early sign of such pathology. Vascular conditions known to cause exercise-related complaints can be classified as non-occlusive or occlusive disease, affecting single or multiple vessels. The main cause of single artery stenosis is celiac artery compression, in which extrinsic compression of the celiac artery by the median arcuate ligament of the diaphragm hampers the inflow of blood $(82,119)$. Critically reduced perfusion through the celiac artery is thought to produce upper abdominal pain in a similar fashion as exercise leads to ischemia-associated abdominal discomfort. This also explains why the pain in celiac artery compression syndrome (CACS) is typically experienced postprandially and during exercise, since a combination of ischemic factors leads to a greater perfusion mismatch within the splanchnic area. While the prevalence of CACS in the general population is still unclear, Mensink et al. found a prevalence of $13.4 \%$ in 320 patients with suspected chronic GI ischemia (82). Other conditions such as splanchnic atherosclerosis may also cause non-occlusive mesenteric ischemia (NOMI), classically leading to postprandial abdominal pain and exercise-induced symptoms. In addition, patients with compromised cardiac and/or pulmonary function, e.g. chronic heart failure or chronic obstructive pulmonary disease (COPD) are suggested to be at risk of developing splanchnic hypoperfusion and the associated intestinal injury, barrier dysfunction and inflammation (113). Since intestinal inflammation may contribute to the state of chronic inflammation that is often seen in these patients, it is essential to avoid an excessive splanchnic response. On the other hand, regular physical exercise in these chronic patients is of important clinical relevance, since it helps to maintain muscle mass and strength, improves cardiovascular fitness, and increases quality of life.

\section{Evaluation of splanchnic perfusion in athletes}

The currently available clinical tools that may be used to identify whether disproportional splanchnic hypoperfusion or ischemia is present during exercise are tonometry and techniques to evaluate the intestinal microcirculation. An overview of the available tools is depicted in Table 1.

Exercise tonometry enables detection of GI ischemia in all symptomatic athletes during 10 min of maximal exercise (plasma lactate $>5.5 \mathrm{mmol} / \mathrm{L}$ ). About $50 \%$ of these symptomatic athletes also showed signs of GI ischemia during $10 \mathrm{~min}$ of exercise at submaximal workload $(3<$ lactate $<5.5 \mathrm{mmol} / \mathrm{L})(124)$. Criteria for positive tonometry outcomes generally differ between centres. Otte et al. considered tonometry to be positive in symptomatic athletes in case of increased luminal $\mathrm{pCO}_{2}$ compared with baseline values, in combination with a gap (gastric-arterial, g-a $)$ $\mathrm{pCO}_{2}>0.8 \mathrm{kPa}$, or for jejunal tonometry gap $($ jejunal-arterial, j-a $) \mathrm{pCO}_{2}>1.4 \mathrm{kPa}(97)$.

Ter Steege et al. used the same criteria to identify ischemia during exercise tonometry, and found $\operatorname{gap}\left(\mathrm{g}_{\mathrm{a}}\right) \mathrm{pCO}_{2}$ levels of 1.1 and $0.5 \mathrm{kPa}$ during 10 minutes of submaximal exercise in 
Table 1: Tools to assess exercise-induced splanchnic hypoperfusion and its sequelae in athletes

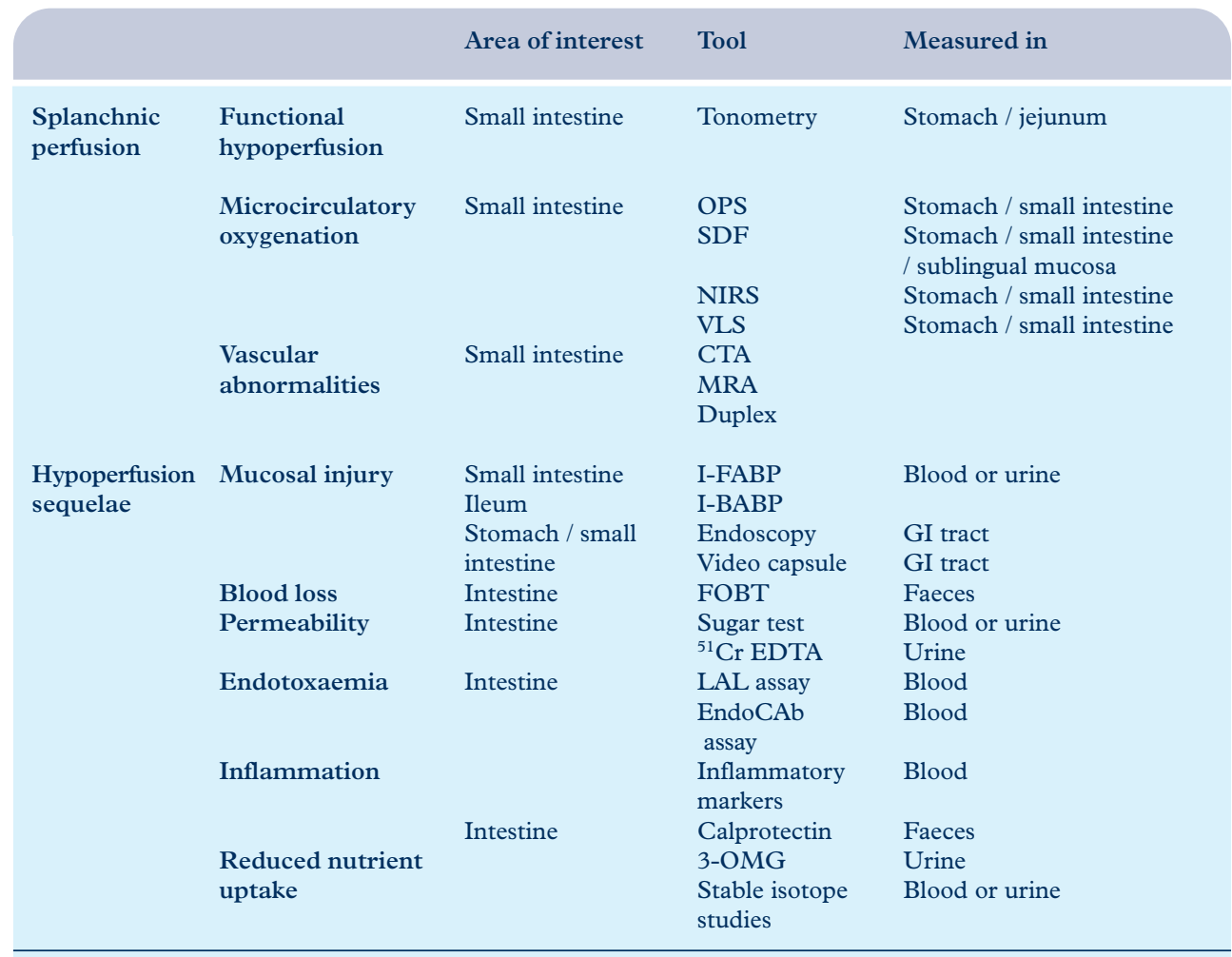

OPS = orhogonal polarization spectral imaging, SDF = sidestream dark field imaging, NIRS = near-infrared spectroscopy, VLS = visible light spectroscopy. CTA = computed tomographic angiography, $\mathrm{MRA}=$ magnetic resonance angiography, I-FABP = intestinal fatty acid binding protein, I-BABP = ileal bile acid binding protein, FOBT = faecal occult blood test, ${ }^{51} \mathrm{Cr}$ EDTA $={ }^{51} \mathrm{Chromium}$ ethylene diamine tetra-acetic acid, LAL assay $=$ limulus amoebocyte lysate assay, EndoCAb assay = endotoxin core antibodies assay, 3-OMG = 3-O-methyl-glucose

symptomatic and asymptomatic athletes, respectively, and 2.5 and $1.7 \mathrm{kPa}$ during 10 minutes exercise increasing to maximum intensity (124). We observed increased gap $\mathrm{p}_{(\mathrm{g}-\mathrm{a})} \mathrm{pCO}_{2}$ levels from -0.85 in rest to $0.85 \mathrm{kPa}$ after 1 hour of cycling at $70 \%$ of the individual athlete's maximal workload capacity $\left(\mathrm{W}_{\text {max }}\right.$ ) without major abdominal complaints (Figure 3A) (135). An alternative way to evaluate exercise-induced ischemia may be to consider the increase in $\operatorname{gap}(\mathrm{g}-\mathrm{a})$ $\mathrm{pCO}_{2}$ from baseline, since physiological baseline differences are common, and cut off points for ischemia may also be individually determined. We propose to include markers of ischemiarelated epithelial injury and GI compromise such as I-FABP and permeability ratios, respectively, to improve the diagnosis of exercise-induced ischemia. The diagnosis of CACS is largely similar to the multidisciplinary work-up of GI ischemia, including gastric exercise tonometry and visualisation of the stenosis, and is described in detail by Mensink et al (82). 
Novel techniques introduced to evaluate the intestinal microcirculation are orthogonal polarization spectral (OPS) imaging $(44,47)$, side stream dark field (SDF) imaging (58), near-infrared spectroscopy (NIRS) and visible light spectroscopy (VLS). These techniques provide information on the mucosal oxygen saturation of haemoglobin in red blood cells of the intestinal microcirculation by emission and detection of backscattered light during endoscopy $(5,81,134)$. This provides a direct measurement of mucosal microcirculatory saturation and simultaneous macroscopic evaluation of the mucosa during upper endoscopy in clinical settings. Analysis of the sublingual microcirculation using the same techniques provides an indirect, less invasive alternative that may prove useful for athletes to monitor the effects of training on GI function. Measurements must be performed before and directly after cessation of exercise, since movement of the diagnostic probe during exercise interferes with the quality of microcirculatory imaging. Data on the use of these techniques in athletes are not yet available. Studies in patients suspected of chronic GI ischemia are promising (134), and sublingual microcirculation measurements were reported to correlate closely with measurements of the intestinal microcirculation in animal studies (139).

If vascular abnormalities or vascular occlusion are considered to explain existing abdominal symptoms that present either at rest or during exercise, imaging of the abdominal vasculature should also be obtained. For this purpose, computed tomography angiography (CTA), classic or magnetic resonance angiography (MRA), or transcutaneous abdominal duplex ultrasound may be used. The advantages and disadvantages of these techniques are beyond the scope of this review, but a useful, comprehensive overview of these techniques with respect to chronic GI ischemia is provided by Mensink et al. (81). Only duplex ultrasound scanning may be used during exercise to establish reduced intestinal perfusion, but reproducibility of this technique in healthy, well-trained athletes is rather poor (103).

Finally, if other serious pathology is suspected that may induce or aggravate abdominal complaints during or after exercise, like inflammatory bowel disease or a Helicobacter pylori infection, endoscopy (in combination with biopsy and/or microcirculatory analysis as described earlier) may be indicated. Video capsule endoscopy provides a non-invasive, elegant alternative for pathology that can be observed macroscopically (22). It remains to be determined if capsule endoscopy can contribute to the assessment of exercise-induced intestinal compromise and whether its use is safe during exercise.

In summary, evaluation of splanchnic perfusion during and after exercise is possible using the functional gastric tonometry test, which could be combined with analysis of circulating I-FABP levels. Furthermore, novel techniques are available to evaluate intestinal microcirculation, and imaging of the splanchnic vasculature may be useful to exclude vascular abnormalities. 


\section{Evaluation of compromised epithelial integrity in athletes}

Circulating plasma I-FABP levels, determined by an enzyme-linked immunosorbent assay (ELISA), can be used during and after exercise to obtain information on intestinal injury (128, 135). In healthy, trained individuals, plasma I-FABP levels doubled from approximately 300 to over $600 \mathrm{pg} / \mathrm{mL}$ during one hour of cycling at $70 \% \mathrm{~W}_{\max }$ (135). In addition, plasma I-FABP levels were found to correlate with exercise-induced splanchnic hypoperfusion determined 20 minutes earlier, emphasizing the suitability of I-FABP as a marker of ischemia-related intestinal injury in athletes (135). Cut off points for differentiation of normal and abnormal circulating I-FABP levels depend on the sensitivity of the test that is used. Ideally, the athlete's baseline I-FABP level is determined (at rest) and subsequent levels measured during exercise should be expressed accordingly. The change in I-FABP levels (i.e. the delta) likely represents a more reliable measure to reflect GI homeostasis during exercise and subsequent post-exercise recovery. The development of a reliable quick-test to evaluate circulating I-FABP levels in capillary blood obtained from a fingertip or ear lobe would greatly increase the applicability of I-FABP as marker of ischemia-related injury. In addition to the analysis of plasma and urinary I-FABP levels for early detection of exercise-induced intestinal injury, intestinal bile-acid binding protein (I-BABP) may be used to provide site-specific information on the ileum (135).

In addition, plasma citrulline level before and after exercise may be determined by high-performance liquid chromatography (HPLC) as a marker of remaining enterocyte mass (27). Similar to I-FABP, the delta of such plasma levels may be used as a parameter of intestinal integrity. Alternatively, the citrulline generation test has been suggested to assess enterocyte function, and may be used athletes during exercise and post-exercise recovery. The citrulline generation test is based on the oral ingestion of a bolus ( $20 \mathrm{~g})$ of alanine-glutamine, and determination of plasma citrulline levels that may change in relation to villous atrophy (104). It remains to be determined whether these tests are useful to assess in exercise-related epithelial injury.

Occult GI blood loss is another feature of exercise-induced intestinal compromise $(8,133)$, and therefore, FOBT may also be used to determine intestinal integrity during and/or immediately after exercise. The most common commercially available FOBT is the Hemoccult test (Beckman Coulter Inc. CA, U.S.A.). A 'positive' test result is obtained if the tested stool sample contains over 2-4 $\mathrm{mL}$ of blood per $100 \mathrm{~g}$ faeces, due to any process that may induce intestinal bleeding, ranging from physical exercise to colorectal carcinoma (129). To avoid false positive results, it is important that subjects adhere to dietary recommendations such as avoiding intake of red meat, raw, peroxide-like fruits and vegetables or vitamin C supplements, may generate false positive outcomes (129). However, it should be noted that the latest FOBTs no longer require such dietary protocols.

Furthermore, among the tests that may be used to assess the impact of exercise on the GI system are tests that aim to assess gut barrier loss. An elegant test to determine the gut barrier function is the sugar-based permeability test, in which the individual drinks a test solution com- 
posed of small $(<200 \mathrm{kD})$ and large $(>200 \mathrm{kD})$ sugar probes. Preferably, the test drink is ingested during, or just after exercise. Urinary and/or plasma concentrations are obtained using HPLC in combination with mass spectometry (HPLC-MS) (136). The urinary excretion of single sugars and the ratio of large and small sugars can be used to assess GI permeability. The advantage of plasma-based permeability analysis is the ability to detect smaller and transient permeability changes that may not be found on urinary analysis (135). The timing of urinary collections and the selection of the sugar probes depend on the specific area of interest. If small intestinal permeability is to be tested, urine should be collected over $0-3$ or $0-5$ hours, using the sugar probes lactulose and L-rhamnose. In contrast, for large intestinal permeability 3-24 or 524 hour urinary collections are used, in combination with two sugar probes such as sucralose and erythritol that are non-degradable by bacteria in the colon (9). A classic alternative for intestinal permeability assessment is the use of ${ }^{51} \mathrm{Cr}$ EDTA) (95), but since this technique is dependent on renal function, which may be changed during strenuous exercise, and because of its radioactivity (32), its use for exercise-induced changes is not recommended.

In addition to permeability tests, loss of intestinal barrier function after strenuous exercise may be determined by analysis of circulating endotoxin levels (64). High levels indicate that the GI mucosa was unable to preserve an effective barrier function, resulting in bacterial translocation from the gut lumen to the circulation. Techniques that may be used to determine whether translocation has occurred are the Limulus Amoebocyte Lysate (LAL) assays, quantifying endotoxin levels in plasma samples, and a test that measures the concentration of endotoxin core antibodies (EndoCAb), with reduced antibody levels reflecting antibody consumption by increased circulating endotoxin. Both assays require sampling prior to exercise and post-exercise to detect changes in endotoxin or antibody levels, respectively. However, both assays may be influenced by confounding factors such as previous endotoxin exposure, and fungi or polynucleotides that may produce false positive results $(50,64)$.

Loss of epithelial integrity in the gut is also reflected by the secretion of inflammatory markers. While determination of circulatory C-reactive protein, white blood cell counts, and neutrophil products may be useful to estimate the whole body response to exercise, no gut-specific information can be derived from such measurements, since other tissues such as muscles are also known to produce pro-inflammatory cytokines during exercise (100). Mucosal injury triggers neutrophils and other inflammatory cells to migrate towards the site of injury, where the neutrophils are activated and release their antimicrobial peptides $(26,33,34,37)$. Hence, more specific information on intestinal inflammation may be obtained from quantification of faecal levels of peptides such as calprotectin, a $36 \mathrm{kD}$-protein with antimicrobial and immunomodulating ability $(37,122,123)$.

Changes in the uptake of nutrients, both during strenuous exercise and in the acute recovery period, remain to be clarified. Active sugar uptake during exercise may be analyzed using 3-O-methyl-glucose (3-OMG), e.g. during permeability analysis (94). In addition, stable isotope 
studies may help to reveal changes in nutrient uptake and possible correlations with exerciseinduced intestinal compromise. The latter enable the use of dietary products present in an average every day diet, thereby providing safe and very relevant information on digestion and absorption of these products (35).

The main features of compromised epithelial integrity during and after exercise are small intestinal injury and increased permeability, which may be determined by analysis of plasma IFABP levels and permeability analysis. An overview of the available tools to assess intestinal compromise in athletes is presented in Table 1.

\section{Future perspectives: evaluation and preventative solutions}

\section{Evaluation of the splanchnic response to exercise in asymptomatic athletes}

Usually, only symptomatic athletes who are limited by GI symptoms during exercise seek help to identify the origin of their symptoms and consequently may adjust their training and feeding strategies based on recommendations provided by the gastroenterologist. However, others may also benefit from increasing their knowledge of the capacities and limitations of the GI system, especially professional athletes and individuals with compromised cardiovascular and/or pulmonary capacity. Individualized information on GI function may be used to modulate the exercise training program, improve feeding strategy, and prevent adverse effects on GI level. Splanchnic responses to exercise may be different in women compared to men (53). Therefore, individualized information could also be used to design training strategies and recommendations for nutritional strategies specifically for female athletes (53).

\section{Solutions to prevent intestinal compromise during exercise}

\section{Training strategies}

Better monitoring of the GI system during training may improve gut function and diminish abdominal discomfort during exercise in symptomatic athletes if training strategies are adapted to GI capacity (124). One of the training adaptations may be to reduce exercise intensity, either temporary or for a longer period of time depending on the individual's outcome, and to maintain heart rates below the level at which symptoms generally seem to occur. It will be interesting to find out whether the application of such a strategy in symptomatic athletes will bring elevated IFABP levels back to physiological levels. If the latter is true, exercise intensity may be adjusted according to the level of I-FABP, and if levels rise above the individual's predetermined threshold, training intensity may be reduced. It may also be possible to precondition or train the gut to withstand prolonged episodes of physical exercise. If the latter is true, athletes may benefit by gradually increasing exercise intensity during a single training session, creating opportunity to establish an equilibrium that fits the altered physiological situation. Studies that include data on specific 
adaptations of the gut to training show that gastric emptying and intestinal transit are increased in highly active individuals compared with less active people (18), and that individuals that practice the intake of food and fluids in training sessions experience significantly less GI distress during competition (125), suggesting that adaptation of the GI tract to physical exercise is possible.

\section{Feeding strategies}

Optimization of feeding strategies reduces abdominal distress in symptomatic athletes $(60,124)$. In addition to a well-balanced every day diet, it is recommended to avoid large meals and the intake of nutritional products that are not easily digested in the 2-3 hours prior to strenuous physical exercise, such as foods with a high fibre, protein, or fat content $(63,110)$. The reason for this may be that postprandially, splanchnic blood flow increases significantly (142). The ingestion of these specific foods is associated with larger decreases in splanchnic vascular resistance compared with easily digestible foods $(66,87)$, which may result in more pronounced splanchnic vasodilatation and increased splanchnic flow. In fact, the ingestion of a liquid test meal $(390 \mathrm{kcal}, 21.6 \mathrm{~g}$ protein, $15.4 \mathrm{~g}$ fat, $41.3 \mathrm{~g}$ carbohydrate ( $\mathrm{CHO})$ ) during the first 10 minutes of a 15 -minute exercise bout on a treadmill at $20 \%$ incline was reported to increase splanchnic blood flow (109), suggesting that oral intake may overrule the exercise-induced decrease in splanchnic circulation. However, the moderate intensity level in this study may explain why exercise-induced hypoperfusion did not overrule the increase in blood flow induced by this large food bolus. Future studies are needed to clarify whether the ingestion of food also interferes with exercise-induced vasoconstriction at higher intensity levels and whether a mismatch in blood flow gives rise to abdominal distress. Currently, athletes are recommended to use small amounts of easily digestible foods in the hours before and during exercise while maintaining euhydration (29). During exercise, $\mathrm{CHO}$ ingestion is recommended, since it improves endurance capacity and performance $(60,61)$. In highly active athletes, the combination of glucose and fructose may be beneficial since it resulted in higher CHO oxidation rates than the ingestion of a single $\mathrm{CHO}$ (62), attenuating the depletion of endogenous energy stores during exercise and stimulating repletion of these stores during acute post-exercise recovery. However, care should be taken to avoid the intake of hyperosmolar fluids, since these have been associated with abdominal distress and hyperosmolar diarrhea during exercise (110).

Although the intake of high-fat products is not recommended in the 2-3 hours prior to and during strenuous physical exercise, lipid-enriched nutrition has been described to attenuate intestinal inflammation, bacterial translocation, and intestinal injury following intestinal hypoperfusion (28). These effects have been assigned to the activation of the autonomic nervous system that increases the splanchnic blood flow via activation of cholecystokinine receptors $(28,118)$. In line, lipid-enriched nutrition ingested in the post-exercise period may improve GI function and reduce the flu-like condition associated with endotoxaemia after strenuous physical exercise by improving post-exercise splanchnic flow.

Nutritional strategies prior to, during and post-exercise should always be tailored to the indi- 
vidual athlete, and trial and error during training sessions will yield the most effective strategy to be used during competition (61). Oral intake should be practiced in training since athletes unaccustomed to ingest fluids or foods during exercise experience significantly more GI complaints upon such intake during a competitive run (125). In addition, the athlete's personal preference for food and fluids should not be neglected as it is a strong determinant for the athlete's tolerance to nutrition during exercise.

\section{Strategies to improve splanchnic perfusion}

Strenuous physical exercise is typically associated with significant splanchnic hypoperfusion, which can be severe enough to impair the structural and functional integrity of the GI tract. In case of significant vascular abnormalities or stenosis due to CACS, revascularization treatment may be required to improve splanchnic perfusion (81). The majority of individuals with exercise-induced splanchnic hypoperfusion will not need revascularization. Numerous less invasive measures are available to improve splanchnic hypoperfusion in the absence of vascular abnormalities. Adequate hydration contributes to splanchnic perfusion maintenance by avoiding systemic hypovolemia. Since there is considerable variability in fluid loss between individuals, customized fluid replacement is recommended to maintain adequate hydration during exercise (115). In addition, specific agents may be used to enhance splanchnic perfusion and improve GI homeostasis during exercise. As stated previously, the splanchnic microvasculature plays a fundamental role in GI homeostasis, and provides an additional target for enhancement of splanchnic flow. One of the important endogenous players that significantly contribute to the vasodilator responses within the intestinal microcirculation is nitric oxide (NO) (52). Interventions that increase the intestinal availability of NO may improve splanchnic perfusion.

Normally, intestinal microvessels vasodilate in response to acetylcholine via an NO-dependent mechanism $(52,138)$. NO synthase (NOS) oxidizes an $\mathrm{N}$-atom of L-arginine to produce L-citrulline and NO. To enable this arginine oxidation, NOS requires the cosubstrate NADPH (nicotinamide adenine dinucleotide phosphate-oxidase), $\mathrm{O}_{2}$, and a number of cofactors (78). Stimulation via acetylcholine increases the cytosolic calcium levels and activates endothelial NOS (eNOS), leading to NO production (78). Of the three identified NOS isoforms, eNOS is reported to be responsible for vascular regulation, particularly in the splanchnic area (78). Regulation of eNOS occurs via guanylyl cyclases (GC), which are transmembrane receptors with an intracellular domain that becomes enzymatically active upon stimulation. The way of activation is dependent on the type of GC, and activation via $\mathrm{NO}$ is one of the possibilities. NO-mediated vasodilatation occurs when the binding of NO to GC induces structural changes in GC. Cyclic guanosine monophosphate (cGMP) production in endothelial cells increases, resulting in intracellular signalling to initiate relaxation of the vascular smooth muscles, promoting local vasodilatation (78).

There are a number of options to upregulate intestinal NO availability that may be of value for athletes experiencing abdominal distress associated with splanchnic hypoperfusion. The two 
Table 2: Promising supplementation strategies to improve splanchnic perfusion in athletes

\begin{tabular}{|c|c|c|c|c|}
\hline Strategy & Potential advantages & Concerns & $\begin{array}{l}\text { Study } \\
\text { population }\end{array}$ & References \\
\hline $\begin{array}{l}\text { (Alanyl) } \\
\text { Glutamine }\end{array}$ & $\begin{array}{l}\text { - Increased NO availability may } \\
\text { improve GI perfusion } \\
\text { - Attenuated intestinal ischemic injury } \\
\text { - Decreased infectious comorbidity }\end{array}$ & & $\begin{array}{l}\text { Animals } \\
\text { Multi trauma } \\
\text { patients }\end{array}$ & $\begin{array}{l}\text { Mondello (86) } \\
\text { Houdijk ( } 56\end{array}$ \\
\hline Arginine & $\begin{array}{l}\text { Increased NO synthesis improves } \\
\text { GI blood flow }\end{array}$ & $\begin{array}{l}\text { High doses } \\
\text { induce GI } \\
\text { distress } \\
\text { (including } \\
\text { nausea, } \\
\text { vomiting, } \\
\text { diarrhea) }\end{array}$ & Athletes & Grimble (46) \\
\hline & $\begin{array}{l}\text { Enhanced tolerance to high-intensity } \\
\text { exercise } \\
\text { - Reduced systolic BP }\end{array}$ & & Athletes & Bailey (3) \\
\hline Citrulline & $\begin{array}{l}\text { Increased arginine pool for } \\
\text { NO synthesis } \\
\text { - Improves nitrogen balance }\end{array}$ & & Animals & Osowska (96) \\
\hline Nitrite & $\begin{array}{l}\text { - Increased NO availability improves } \\
\text { GI perfusion } \\
\text { - Cytoprotective } \\
\text { - Decreased ROS formation }\end{array}$ & & $\begin{array}{l}\text { Animals } \\
\text { Animals }\end{array}$ & $\begin{array}{l}\text { Petersson (105) } \\
\text { Shiva (117) }\end{array}$ \\
\hline Nitrate & $\begin{array}{l}\text { - Increased NO availability improves } \\
\text { GI perfusion } \\
\text { - Cytoprotective } \\
\text { - Decreased ROS formation } \\
\text { - Impranced tolerance to heavy exercise } \\
\end{array}$ & & $\begin{array}{l}\text { Animals } \\
\text { Animals } \\
\text { Athletes }\end{array}$ & $\begin{array}{l}\text { Petersson (105) } \\
\text { Shiva (117) } \\
\text { Cermak (20) } \\
\text { Vanhatalo (137) } \\
\text { Bailey (2) }\end{array}$ \\
\hline
\end{tabular}

BP, blood pressure - ROS, reactive oxygen species - NO, nitric oxide - GI, gastrointestinal.

experimental interventions that will be discussed in this review are NOS-dependent (glutaminearginine-citrulline) and NOS-independent (nitrate-nitrite) supplementation. An overview of these potentially useful strategies is given in Table 2. It is important to bear in mind that while these interventions may improve splanchnic perfusion, it is possible that this will lead to hypoperfusion of other tissues such as the active muscles, which may deteriorate athletic performance. Additionally, since most of these strategies are still in the experimental phase, it remains to be determined whether the use of the described supplements by athletes is safe. Future studies are warranted to clarify these issues. 


\section{Glutamine, arginine and citrulline supplementation}

Glutamine plays an important role in the arginine-NO pathway, as it can be converted in the intestine into citrulline, the precursor of arginine (131). Arginine is the sole natural precursor for the synthesis of NO (55), and can be converted to NO and citrulline in the intestine or metabolized in the liver into urea and ornithine by arginase. Citrulline is synthesized and released into the circulation, from which it is taken up by the kidneys, and converted back into arginine for production of $\mathrm{NO}$ and other amino acids (84). The supplementation of glutamine in patients resulted in enhanced citrulline uptake and higher arginine levels $(56,72)$. However, the outcome and positive effects on arginine production depend on the route of administration and the molecular form of the supplemented glutamine (140). Parenteral glutamine supplementation resulted in higher plasma arginine levels compared to enteral supplementation (72). Unfortunately, nutritional enrichment with glutamine is complicated due to the relative aqueous instability (140). A promising alternative is supplementation of the dipeptide form, alanyl-glutamine, a stable alternative in aqueous solutions with the same positive effects on arginine plasma availability (57). Glutamine supplementation showed no adverse events even in high dosage (93), making the latter a promising alternative to enhance the arginine-NO production in the splanchnic vascular bed during abdominal distress associated with splanchnic hypoperfusion.

To improve arginine availability for NO synthesis, oral arginine supplementation was suggested, but controversy exists on the results of arginine supplementation (12). Arginine supplementation has been described to result in elevated NO synthesis in the hepatosplanchnic area in human and animal studies supplementation $(12,19,106)$. Blood flow in the porcine portal vein increased (106), while systemic cardiovascular variables such as mean arterial pressure and cardiac index did not deteriorate $(73,106)$. In fact, a single $500-\mathrm{mL}$ drink containing $6 \mathrm{~g}$ of arginine significantly reduced the $\mathrm{O}_{2}$ cost of the athletes during moderate-intensity exercise, and enhances high-intensity exercise tolerance (3), suggesting improved cardiopulmonary function. On the other hand, inability to increase porcine mesenteric blood flow by enteral supplementation of arginine has also been reported (108). Furthermore, high doses of arginine have been reported to cause osmotic diarrhoea exercise (46). Therefore, it can be concluded that the effect of arginine supplementation on the splanchnic vascular bed in man, especially in athletes, remains to be clarified.

Citrulline, derived from glutamine conversion, can also be converted to arginine and used for NO synthesis after entering the systemic circulation. Furthermore, it is not subjected to extensive hepatic metabolisation like arginine, making it a promising exogenous source of NO, possibly enabling local vasodilatation and improved splanchnic blood flow. Comparing oral citrulline to oral arginine supplementation, oral citrulline intake is more efficient in increasing body arginine levels (96) and has a higher bioavailability (85). Furthermore, high doses of citrulline do not cause osmotic diarrhoea, whereas arginine does $(46,75)$. Citrulline has been demonstrated to increase arginine availability for NO production (96), but whether the latter will improve splanchnic perfusion and reduce GI distress in athletes remains to be clarified. 


\section{Nitrate and nitrite supplementation}

An alternative, NOS-independent pathway to increase NO availability for vasodilatation is to generate $\mathrm{NO}$ is via nitrate and nitrite, which involves the reduction of ingested nitrate to nitrite and NO, particularly in acidic or hypoxic conditions. Nitrate is normally ingested via food and drinking water, and via the ingestion of mainly green leafy vegetables such as lettuce, spinach, as well as beetroot $(74,137)$. After ingestion much of the nitrate is reduced to nitrite by commensal bacteria in the saliva of the oral cavity, while the remaining nitrate enters the enterosalivary pathway after absorption in the upper GI tract. The nitrite formed by the bacteria, is reduced to NO upon entering the acidic environment of the stomach, a process that is enhanced by the presence of vitamin C and polyphenols (75). A small part of the ingested nitrate and nitrite is absorbed in the small intestine and converted to NO in blood and tissue under physiological acidic and hypoxic conditions (75).

The potency of NO to produce vasodilatation is enhanced under conditions of hypoxia or metabolic stress such as strenuous physical exercise $(75,83)$. Nitrate and nitrite should preferably be supplemented per os, for example as a vegetable juice, since such a vegetable juice also contains vitamin $\mathrm{C}$ necessary for the reduction of nitrite to $\mathrm{NO}$ and maybe even more important, it is the physiological route of nutrient ingestion. Animal studies have demonstrated that both nitrate and nitrite, administered orally and endoluminally, respectively, increased gastric mucosal blood flow (105). Supplementation of nitrate and nitrite was also demonstrated to act cytoprotective and decrease formation of reactive oxygen species (117). And recently, dietary nitrate supplementation (ca. 8 to $11 \mathrm{mmol}$ nitrate in beetroot juice as a single dose or for 6 consecutive days) enhanced tolerance to moderate to high-intensity exercise $(20,137)$, and improved time-trial performance (20). All together, these data suggest that nitrate ingestion during or immediately after exercise can stimulate splanchnic perfusion, which may reduce intestinal injury and prevent loss of intestinal barrier function triggered by hypoperfusion during exercise. As such, intake of dietary nitrate could prevent exercise-related GI problems.

\section{Avoidance of medication that may compromise the GI system}

It is of utmost importance to increase the awareness of athletes and trainers towards the deleterious effects of drugs that may compromise the GI system. High numbers of athletes have been reported to use analgesics to relieve existing or anticipated pain (45). Whereas the use of specific analgesics may paradoxically aggravate GI distress and cause significant small intestinal injury especially during exercise (unpublished data). The use of non-selective non-steroidal anti-inflammatory drugs (NSAIDs), has been associated with a 3 to 5 fold increased risk of upper gastrointestinal complications, mucosal bleeding, or perforation compared to no medication (39). Data indicate that these NSAID-induced complications are the result of two detrimental mechanisms: inhibition of cyclo-oxygenase (COX)-1 reduces blood flow in the upper GI region, whereas inhibition of COX-2 promotes adherence of neutrophils to the vascular endothelium (40). Further- 
more, NSAIDs may interfere with NO production and inhibit the formation of nitrate via regulation of the NFKB pathway (78), thereby further compromising GI homeostasis during exercise-induced hypoperfusion. Last of all, studies have demonstrated that if active (chronic) intestinal inflammation is present, NSAID-induced COX inhibition may result in vasoconstriction $(6,52)$. We consider that the latter in combination with exercise-induced splanchnic hypoperfusion may account for the exacerbation of intestinal injury in athletes using NSAIDs.

\section{Future perspectives}

Although our knowledge on the role of GI function in relation to exercise has greatly improved over the past decades, future studies are warranted. One of the main issues to be clarified is the significance of exercise-induced hypoperfusion and GI integrity loss for digestive and absorptive processes. Consequently, the tools described previously should be further developed to enable easy, non-invasive monitoring of the intestinal response to exercise by athletes.

Future studies will reveal whether it is feasible to obtain individualized information on GI physiology during and upon cessation of exercise and to use this information to improve training and nutritional strategies to match cardiovascular, musculoskeletal and GI capacities, without significantly compromising of any of these systems. Such individualized information may help symptomatic athletes to increase understanding of the nature of their symptoms, and may also assist professional athletes and patients with compromised cardiopulmonary function to improve athletic performance.

Despite the large number of GI symptoms known to occur during exercise, athletes and trainers pay little attention to improving GI function. Future studies may provide clarification on the potential preventative and therapeutic interventions that may be used for this purpose.

\section{CONCLUSIONS}

The extensive plasticity of the gut is generally sufficient to meet the requirements imposed by strenuous physical exercise. However, redistribution of blood away from the splanchnic bed towards active muscle tissue, the cardiopulmonary system, and skin can strongly affect the GI system negatively. Exercise-induced splanchnic hypoperfusion and the subsequent loss of epithelial integrity may lead to the onset of disturbing GI symptoms. However, even in the absence of such symptoms, athletes may want to consider adjusting training and nutritional strategies in terms of the type, amount, and timing, to meet nutritional needs while taking into account existing GI compromise caused by prolonged exercise-induced splanchnic hypoperfusion. 


\section{REFERENCES}

1. Ayabe T, Satchell DP, Wilson CL, Parks WC, Selsted ME, and Ouellette AJ. Secretion of microbicidal $\alpha$-defensins by intestinal Paneth cells in response to bacteria. Nat Immunol 1: 113-118, 2000.

2. Bailey SJ, Winyard P, Vanhatalo A, Blackwell JR, Dimenna FJ, Wilkerson DP, Tarr J, Benjamin N, and Jones AM. Dietary nitrate supplementation reduces the $\mathrm{O}_{2}$ cost of low-intensity exercise and enhances tolerance to highintensity exercise in humans. F Appl Physiol 107: 1144-1155, 2009.

3. Bailey SJ, Winyard PG, Vanhatalo A, Blackwell JR, DiMenna FJ, Wilkerson DP, and Jones AM. Acute L-arginine supplementation reduces the $\mathrm{O} 2$ cost of moderate-intensity exercise and enhances high-intensity exercise tolerance. F Appl Physiol 109: 1394-1403, 2010.

4. Beaumont AC, and Teare JP. Subtotal colectomy following marathon running in a female patient. $\mathcal{F} R$ Soc Med 84: 439-440, 1991.

5. Benaron DA, Parachikov IH, Cheong WF, Friedland S, Rubinsky BE, Otten DM, Liu FW, Levinson CJ, Murphy AL, Price JW, TalmiY, Weersing JP, Duckworth JL, Horchner UB, and Kermit EL. Design of a visible-light spectroscopy clinical tissue oximeter. F Biomed Opt 10: 44005, 2005.

6. Berg DJ, Zhang J, Weinstock JV, Ismail HF, Earle KA, Alila H, Pamukcu R, Moore S, and Lynch RG. Rapid development of colitis in NSAID-treated IL-10-deficient mice. Gastroenterology 123: 1527-1542, 2002.

7. Berkes J, Viswanathan VK, Savkovic SD, and Hecht G. Intestinal epithelial responses to enteric pathogens: effects on the tight junction barrier, ion transport, and inflammation. Gut 52: 439-451, 2003.

8. Bi L, and Triadafilopoulos G. Exercise and gastrointestinal function and disease: an evidence-based review of risks and benefits. Clin Gastroenterol Hepatol 1: 345-355, 2003.

9. Bjarnason I, MacPherson A, and Hollander D. Intestinal permeability: an overview. Gastroenterology 108: 1566$1581,1995$.

10. Blikslager AT. Life in the Gut without oxygen: adaptive mechanisms and inflammatory bowel disease. Gastroenterology 134: 346-348, 2008.

11. Blikslager AT, Moeser AJ, Gookin JL, Jones SL, and Odle J. Restoration of barrier function in injured intestinal mucosa. Physiol Rev 87: 545-564, 2007.

12. Bode-Boger SM, Boger RH, Galland A, Tsikas D, and Frolich JC. L-arginine-induced vasodilation in healthy humans: pharmacokinetic-pharmacodynamic relationship. Br f Clin Pharmacol 46: 489-497, 1998.

13. Bosenberg AT, Brock-Utne JG, Gaffin SL, Wells MT, and Blake GT. Strenuous exercise causes systemic endotoxemia. F Appl Physiol 65: 106-108, 1988.

14. Bradley SE, Childs AW, Combes B, Cournand A, Wade OL, and Wheeler HO. The effect of exercise on the splanchnic blood flow and splanchnic blood volume in normal man. Clin Sci (Lond) l 15: 457-463, 1956.

15. Brouns F, Nieuwenhoven M, Jeukendrup A, and Marken Lichtenbelt W. Functional foods and food supplements for athletes: from myths to benefit claims substantiation through the study of selected biomarkers. Br $\mathcal{F}$ Nutr 88 Suppl 2: S177-186, 2002.

16. Brown BP, Ketelaar MA, Schulze-Delrieu K, Abu-Yousef MM, and Brown CK. Strenuous exercise decreases motility and cross-sectional area of human gastric antrum. A study using ultrasound. Digestive diseases and sciences 39: 940-945, 1994.

17. Camus G, Deby-Dupont G, Deby C, Juchmes-Ferir A, Pincemail J, and Lamy M. Inflammatory response to strenuous muscular exercise in man. Mediators Inflamm 2: 335-342, 1993.

18. Carrio I, Estorch M, Serra-Grima R, Ginjaume M, Notivol R, Calabuig R, and Vilardell F. Gastric emptying in marathon runners. Gut 30: 152-155, 1989.

19. Castillo L, Chapman TE, Yu YM, Ajami A, Burke JF, and Young VR. Dietary arginine uptake by the splanchnic region in adult humans. Am F Physiol 265: E532-539, 1993.

20. Cermak NM, Gibala MJ, and van Loon LJ. Nitrate Supplementation's Improvement of 10-km Time-Trial Performance in Trained Cyclists. Int F Sport Nutr Exerc Metab 22: 64-71, 2012.

21. Choi SC, Choi SJ, Kim JA, Kim TH, Nah YH, Yazaki E, and Evans DF. The role of gastrointestinal endoscopy in long-distance runners with gastrointestinal symptoms. European journal of gastroenterology \& hepatology 13: 1089-1094, 2001.

22. Ciuti G, Menciassi A, and Dario P. Capsule endoscopy: from current achievements to open challenges. IEEE Rev Biomed Eng 4: 59-72, 2011.

23. Clausen JP. Effect of physical training on cardiovascular adjustments to exercise in man. Physiological reviews 57: 779-815, 1977.

24. Coggan AR, and Swanson SC. Nutritional manipulations before and during endurance exercise: effects on performance. Medicine and science in sports and exercise 24: S331-335, 1992.

25. Cooper BT, Douglas SA, Firth LA, Hannagan JA, and Chadwick VS. Erosive gastritis and gastrointestinal bleeding in a female runner. Prevention of the bleeding and healing of the gastritis with $\mathrm{H} 2$-receptor antagonists. Gastroenterology 92: 2019-2023, 1987. 
26. Costa F, Mumolo MG, Bellini M, Romano MR, Ceccarelli L, Arpe P, Sterpi C, Marchi S, and Maltinti G. Role of faecal calprotectin as non-invasive marker of intestinal inflammation. Dig Liver Dis 35: 642-647, 2003.

27. Crenn P, Vahedi K, Lavergne-Slove A, Cynober L, Matuchansky C, and Messing B. Plasma citrulline: A marker of enterocyte mass in villous atrophy-associated small bowel disease. Gastroenterology 124: 1210-1219, 2003.

28. de Haan JJ, Lubbers T, Hadfoune M, Luyer MD, Dejong CH, Buurman WA, and Greve JW. Postshock intervention with high-lipid enteral nutrition reduces inflammation and tissue damage. Annals of surgery 248: 842848, 2008.

29. de Oliveira EP, and Burini RC. Food-dependent, exercise-induced gastrointestinal distress. J Int Soc Sports Nutr 8: $12,2011$.

30. Derikx JP, Matthijsen RA, de Bruine AP, van Bijnen AA, Heineman E, van Dam RM, Dejong CH, and Buurman WA. Rapid reversal of human intestinal ischemia-reperfusion induced damage by shedding of injured enterocytes and reepithelialisation. PLoS ONE 3: e3428, 2008.

31. Dommett RM, Klein N, and Turner MW. Mannose-binding lectin in innate immunity: past, present and future. Tissue Antigens 68: 193-209, 2006.

32. Elia M, Behrens R, Northrop C, Wraight P, and Neale G. Evaluation of mannitol, lactulose and 51Cr-labelled ethylenediaminetetra-acetate as markers of intestinal permeability in man. Clin Sci (Lond) l 73: 197-204, 1987.

33. Fagerhol MK. Calprotectin, a faecal marker of organic gastrointestinal abnormality. Lancet 356: 1783-1784, 2000.

34. Fagerhol MK, Nielsen HG, Vetlesen A, Sandvik K, and Lyberg T. Increase in plasma calprotectin during longdistance running. Scandinavian journal of clinical and laboratory investigation 65: 211-220, 2005.

35. Fielding B. Tracing the fate of dietary fatty acids: metabolic studies of postprandial lipaemia in human subjects. The Proceedings of the Nutrition Society 70: 342-350, 2011.

36. Fink MP DR. Epithelial barrier dysfunction: a unifying theme to explain the pathogenesis of multiple organ dysfunction at the cellular level. Critical Care Clinic 21: 177-196, 2005.

37. Foell D, Wittkowski H, and Roth J. Monitoring disease activity by stool analyses: from occult blood to molecular markers of intestinal inflammation and damage. Gut 58: 859-868, 2009.

38. Fordtran JS, and Saltin B. Gastric emptying and intestinal absorption during prolonged severe exercise. $\mathcal{F} A p p l$ Physiol 23: 331-335, 1967.

39. Gabriel SE, Jaakkimainen L, and Bombardier C. Risk for serious gastrointestinal complications related to use of nonsteroidal anti-inflammatory drugs. A meta-analysis. Ann Intern Med 115: 787-796, 1991.

40. Garcia Rodriguez LA, and Barreales Tolosa L. Risk of upper gastrointestinal complications among users of traditional NSAIDs and COXIBs in the general population. Gastroenterology 132: 498-506, 2007.

41. Gil SM, Yazaki E, and Evans DF. Aetiology of running-related gastrointestinal dysfunction. How far is the finishing line? Sports medicine (Auckland, NZ 26: 365-378, 1998.

42. Gisolfi CV. Is the GI System Built For Exercise? News Physiol Sci 15: 114-119, 2000.

43. Gisolfi CV, Spranger KJ, Summers RW, Schedl HP, and Bleiler TL. Effects of cycle exercise on intestinal absorption in humans. F Appl Physiol 71: 2518-2527, 1991.

44. Goedhart PT, Khalilzada M, Bezemer R, Merza J, and Ince C. Sidestream Dark Field (SDF) imaging: a novel stroboscopic LED ring-based imaging modality for clinical assessment of the microcirculation. Opt Express 15 : 15101-15114, 2007.

45. Gorski T, Cadore EL, Pinto SS, da Silva EM, Correa CS, Beltrami FG, and Kruel LF. Use of NSAIDs in triathletes: prevalence, level of awareness and reasons for use. Br F Sports Med 45: 85-90, 2011.

46. Grimble GK. Adverse gastrointestinal effects of arginine and related amino acids. $\mathcal{F}$ Nutr 137: 1693S-1701S, 2007.

47. GronerW, Winkelman JW, Harris AG, Ince C, Bouma GJ, Messmer K, and Nadeau RG. Orthogonal polarization spectral imaging: a new method for study of the microcirculation. Nat Med 5: 1209-1212, 1999.

48. Grootjans J, Hodin CM, de Haan JJ, Derikx JP, Rouschop KM, Verheyen FK, van Dam RM, Dejong CH, Buurman WA, and Lenaerts K. Level of activation of the unfolded protein response correlates with Paneth cell apoptosis in human small intestine exposed to ischemia/reperfusion. Gastroenterology 140: 529-539 e523, 2011.

49. Grootjans J, Lenaerts K, Derikx JP, Matthijsen RA, de Bruine AP, van Bijnen AA, van Dam RM, Dejong CH, and Buurman WA. Human intestinal ischemia-reperfusion-induced inflammation characterized: experiences from a new translational model. The American journal of pathology 176: 2283-2291, 2010.

50. Grootjans J, Thuijls G, Verdam F, Derikx JP, Lenaerts K, and Buurman WA. Non-invasive assessment of barrier integrity and function of the human gut. World F Gastrointest Surg 2: 61-69, 2010.

51. Hallback DA, Jodal M, Mannischeff $M$, and Lundgren O. Tissue osmolality in intestinal villi of four mammals in vivo and in vitro. Acta Physiol Scand 143: 271-277, 1991.

52. Hatoum OA, Binion DG, Otterson MF, and Gutterman DD. Acquired microvascular dysfunction in inflammatory bowel disease: Loss of nitric oxide-mediated vasodilation. Gastroenterology 125: 58-69, 2003.

53. Hausswirth C, and Le MeurY. Physiological and nutritional aspects of post-exercise recovery: specific recommendations for female athletes. Sports medicine (Auckland, NZ 41: 861-882, 2011. 
54. Heer M, Repond F, Hany A, Sulser H, Kehl O, and Jager K. Acute ischaemic colitis in a female long distance runner. Gut 28: 896-899, 1987.

55. Hibbs JB, Jr., Taintor RR, and Vavrin Z. Macrophage cytotoxicity: role for L-arginine deiminase and imino nitrogen oxidation to nitrite. Science 235: 473-476, 1987.

56. Houdijk AP, Rijnsburger ER, Jansen J, Wesdorp RI, Weiss JK, McCamish MA, TeerlinkT, Meuwissen SG, Haarman HJ, Thijs LG, and van Leeuwen PA. Randomised trial of glutamine-enriched enteral nutrition on infectious morbidity in patients with multiple trauma. Lancet 352: 772-776, 1998.

57. Hubl W, Druml W, Langer K, and Lochs H. Influence of molecular structure and plasma hydrolysis on the metabolism of glutamine-containing dipeptides in humans. Metabolism 38: 59-62, 1989.

58. Ince C. The microcirculation is the motor of sepsis. Critical care (London, England) 9 Suppl 4: S13-19, 2005.

59. Ivanov AI, McCall IC, Parkos CA, and Nusrat A. Role for actin filament turnover and a myosin II motor in cytoskeleton-driven disassembly of the epithelial apical junctional complex. Molecular Biology of the Cell 15: 2639-2651, 2004.

60. Jeukendrup A, Brouns F, Wagenmakers AJ, and Saris WH. Carbohydrate-electrolyte feedings improve $1 \mathrm{~h}$ time trial cycling performance. International journal of sports medicine 18: 125-129, 1997.

61. Jeukendrup AE, Hopkins S, Aragon-Vargas LF, and Hulston C. No effect of carbohydrate feeding on $16 \mathrm{~km}$ cycling time trial performance. European journal of applied physiology 104: 831-837, 2008.

62. Jeukendrup AE, and Jentjens R. Oxidation of carbohydrate feedings during prolonged exercise: current thoughts, guidelines and directions for future research. Sports medicine (Auckland, NZ 29: 407-424, 2000.

63. Jeukendrup AE, Saris WH, Schrauwen P, Brouns F, and Wagenmakers AJ. Metabolic availability of mediumchain triglycerides coingested with carbohydrates during prolonged exercise. F Appl Physiol 79: 756-762, 1995.

64. Jeukendrup AE, Vet-Joop K, Sturk A, Stegen JH, Senden J, Saris WH, and Wagenmakers AJ. Relationship between gastro-intestinal complaints and endotoxaemia, cytokine release and the acute-phase reaction during and after a long-distance triathlon in highly trained men. Clin Sci (Lond) l 98: 47-55, 2000.

65. Kenney WL, and Ho CW. Age alters regional distribution of blood flow during moderate-intensity exercise. $\mathcal{F}$ Appl Physiol 79: 1112-1119, 1995.

66. Kircher P, Lang J, Blum J, Gaschen F, Doherr M, Sieber C, and Gaschen L. Influence of food composition on splanchnic blood flow during digestion in unsedated normal dogs: a Doppler study. Vet f 166: 265-272, 2003.

67. Kolkman JJ, Groeneveld AB, van der Berg FG, Rauwerda JA, and Meuwissen SG. Increased gastric $\mathrm{PCO}_{2}$ during exercise is indicative of gastric ischaemia: a tonometric study. Gut 44: 163-167, 1999.

68. Lakatta EG. Catecholamines and cardiovascular function in aging. Endocrinol Metab Clin North Am 16: 877891, 1987.

69. Lambert GP. Intestinal barrier dysfunction, endotoxemia, and gastrointestinal symptoms: the 'canary in the coal mine' during exercise-heat stress? Med Sport Sci 53: 61-73, 2008.

70. Lambert GP, Lang J, Bull A, Pfeifer PC, Eckerson J, Moore G, Lanspa S, and O'Brien J. Fluid restriction during running increases GI permeability. Int F Sports Med 29: 194-198, 2008.

71. Lang JA, Gisolfi CV, and Lambert GP. Effect of exercise intensity on active and passive glucose absorption. Int J Sport Nutr Exerc Metab 16: 485-493, 2006.

72. Ligthart-Melis GC, van de Poll MC, Boelens PG, Dejong CH, Deutz NE, and van Leeuwen PA. Glutamine is an important precursor for de novo synthesis of arginine in humans. Am f Clin Nutr 87: 1282-1289, 2008.

73. Luiking YC, Poeze M, Ramsay G, and Deutz NE. The role of arginine in infection and sepsis. FPEN F Parenter Enteral Nutr 29: S70-74, 2005.

74. Lundberg JO, Weitzberg E, Cole JA, and Benjamin N. Nitrate, bacteria and human health. Nat Rev Microbiol 2: 593-602, 2004.

75. Lundberg JO, Weitzberg E, and Gladwin MT. The nitrate-nitrite-nitric oxide pathway in physiology and therapeutics. Nat Rev Drug Discov 7: 156-167, 2008.

76. Marchbank T, Davison G, Oakes JR, Ghatei MA, Patterson M, Moyer MP, and Playford RJ. The nutriceutical bovine colostrum truncates the increase in Gut permeability caused by heavy exercise in athletes. Am J Physiol Gastrointest Liver Physiol 300: G477-484, 2011.

77. Marchiando AM, Shen L, Graham WV, Edelblum KL, Duckworth CA, Guan Y, Montrose MH, Turner JR, and Watson AJ. The epithelial barrier is maintained by in vivo tight junction expansion during pathologic intestinal epithelial shedding. Gastroenterology 140: 1208-1218 e1201-1202, 2011.

78. Martin MJ, Jimenez MD, and Motilva V. New issues about nitric oxide and its effects on the gastrointestinal tract. Curr Pharm Des 7: 881-908, 2001.

79. Matheson PJ, Wilson MA, and Garrison RN. Regulation of intestinal blood flow. F Surg Res 93: 182-196, 2000.

80. Matthijsen RA, Derikx JP, Steffensen R, van Dam RM, Dejong CH, and Buurman WA. Mannose-binding lectin null alleles are associated with preserved epithelial cell integrity following intestinal ischemia reperfusion in man. Molecular Immunology 46: 2244-2248, 2009. 
81. Mensink PB, Moons LM, and Kuipers EJ. Chronic gastrointestinal ischaemia: shifting paradigms. Gut 60: 722-737, 2011.

82. Mensink PB, van Petersen AS, Kolkman JJ, Otte JA, Huisman AB, and Geelkerken RH. Gastric exercise tonometry: the key investigation in patients with suspected celiac artery compression syndrome. F Vasc Surg 44: 277-281, 2006.

83. Modin A, Bjorne H, Herulf M, Alving K, Weitzberg E, and Lundberg JO. Nitrite-derived nitric oxide: a possible mediator of 'acidic-metabolic' vasodilation. Acta Physiol Scand 171: 9-16, 2001.

84. Moinard C, and Cynober L. Citrulline: a new player in the control of nitrogen homeostasis. F Nutr 137: 1621S1625S, 2007.

85. Moinard C, Nicolis I, Neveux N, Darquy S, Benazeth S, and Cynober L. Dose-ranging effects of citrulline administration on plasma amino acids and hormonal patterns in healthy subjects: the Citrudose pharmacokinetic study. Br F Nutr 99: 855-862, 2008.

86. Mondello S, Galuppo M, Mazzon E, Domenico I, Mondello P, Carmela A, and Cuzzocrea S. Glutamine treatment attenuates the development of ischaemia/reperfusion injury of the gut. Eur F Pharmacol 643: 304-315, 2010.

87. Moneta GL, Taylor DC, Helton WS, Mulholland MW, and Strandness DE, Jr. Duplex ultrasound measurement of postprandial intestinal blood flow: effect of meal composition. Gastroenterology 95: 1294-1301, 1988.

88. Moses FM. The effect of exercise on the gastrointestinal tract. Sports medicine (Auckland, NZ 9: 159-172, 1990.

89. Moses FM. Exercise-associated intestinal ischemia. Curr Sports Med Rep 4: 91-95, 2005.

90. Moses FM. Gastrointestinal bleeding and the athlete. The American journal of gastroenterology 88: 1157-1159, 1993.

91. Mythen MG. Postoperative gastrointestinal tract dysfunction. Anesthesia and analgesia 100: 196-204, 2005.

92. Nielsen HB, Svendsen LB, Jensen TH, and Secher NH. Exercise-induced gastric mucosal acidosis. Medicine and science in sports and exercise 27: 1003-1006, 1995.

93. Novak F, Heyland DK, Avenell A, Drover JW, and Su X. Glutamine supplementation in serious illness: a systematic review of the evidence. Critical care medicine 30: 2022-2029, 2002.

94. Ohri SK, Somasundaram S, Koak Y, Macpherson A, Keogh BE, Taylor KM, Menzies IS, and Bjarnason I. The effect of intestinal hypoperfusion on intestinal absorption and permeability during cardiopulmonary bypass. Gastroenterology 106: 318-323, 1994.

95. Oktedalen O, Lunde OC, Opstad PK, Aabakken L, and Kvernebo K. Changes in the gastrointestinal mucosa after long-distance running. Scand F Gastroenterol 27: 270-274, 1992.

96. Osowska S, Moinard C, Neveux N, Loi C, and Cynober L. Citrulline increases arginine pools and restores nitrogen balance after massive intestinal resection. Gut 53: 1781-1786, 2004.

97. Otte JA, Geelkerken RH, Oostveen E, Mensink PB, Huisman AB, and Kolkman JJ. Clinical impact of gastric exercise tonometry on diagnosis and management of chronic gastrointestinal ischemia. Clin Gastroenterol Hepatol 3: 660-666, 2005.

98. Otte JA, Oostveen E, Geelkerken RH, Groeneveld AB, and Kolkman JJ. Exercise induces gastric ischemia in healthy volunteers: a tonometry study. $\mathcal{F}$ Appl Physiol 91: 866-871, 2001.

99. Pals KL, Chang RT, Ryan AJ, and Gisolfi CV. Effect of running intensity on intestinal permeability. $\mathcal{F} A p p l$ Physiol 82: 571-576, 1997.

100. Pedersen BK. Edward F. Adolph distinguished lecture: muscle as an endocrine organ: IL-6 and other myokines. f Appl Physiol 107: 1006-1014, 2009.

101. Pelsers MM, Hermens WT, and Glatz JF. Fatty acid-binding proteins as plasma markers of tissue injury. Clinica chimica acta; international journal of clinical chemistry 352: 15-35, 2005.

102. Peters HP, Bos M, Seebregts L, Akkermans LM, van Berge Henegouwen GP, Bol E, Mosterd WL, and de Vries WR. Gastrointestinal symptoms in long-distance runners, cyclists, and triathletes: prevalence, medication, and etiology. The American journal of gastroenterology 94: 1570-1581, 1999.

103. Peters HP, de Leeuw D, Lapham RC, Bol E, Mosterd WL, and de Vries WR. Reproducibility of ultrasound blood flow measurement of the superior mesenteric artery before and after exercise. International journal of sports medicine 22: 245-249, 2001.

104. Peters JH, Wierdsma NJ, Teerlink T, van Leeuwen PA, Mulder CJ, and van Bodegraven AA. The citrulline generation test: proposal for a new enterocyte function test. Aliment Pharmacol Ther 27: 1300-1310, 2008.

105. Petersson J, Phillipson M, Jansson EA, Patzak A, Lundberg JO, and Holm L. Dietary nitrate increases gastric mucosal blood flow and mucosal defense. Am F Physiol Gastrointest Liver Physiol 292: G718-724, 2007.

106. Poeze M, Bruins MJ, Kessels F, Luiking YC, Lamers WH, and Deutz NE. Effects of L-arginine pretreatment on nitric oxide metabolism and hepatosplanchnic perfusion during porcine endotoxemia. Am f Clin Nutr 93: 1237-1247, 2011. 
107. Porter AM. Do some marathon runners bleed into the gut? Br Med f (Clin Res Ed) 287: 1427, 1983.

108. Puiman PJ, Stoll B, van Goudoever JB, and Burrin DG. Enteral arginine does not increase superior mesenteric arterial blood flow but induces mucosal growth in neonatal pigs. F Nutr 141: 63-70, 2011.

109. Qamar MI, and Read AE. Effects of exercise on mesenteric blood flow in man. Gut 28: 583-587, 1987.

110. Rehrer NJ, Wagenmakers AJ, Beckers EJ, Halliday D, Leiper JB, Brouns F, Maughan RJ, Westerterp K, and Saris WH. Gastric emptying, absorption, and carbohydrate oxidation during prolonged exercise. F Appl Physiol 72: 468-475, 1992.

111. Rowell LB, Blackmon JR, and Bruce RA. Indocyanine Green Clearance and Estimated Hepatic Blood Flow During Mild to Maximal Exercise in Upright Man. $\mathcal{F}$ Clin Invest 43: 1677-1690, 1964.

112. Ryan AJ, Lambert GP, Shi X, Chang RT, Summers RW, and Gisolfi CV. Effect of hypohydration on gastric emptying and intestinal absorption during exercise. F Appl Physiol 84: 1581-1588, 1998.

113. Sandek A, Bauditz J, Swidsinski A, Buhner S, Weber-Eibel J, von Haehling S, Schroedl W, Karhausen T, Doehner W, Rauchhaus M, Poole-Wilson P, Volk HD, Lochs H, and Anker SD. Altered intestinal function in patients with chronic heart failure. J Am Coll Cardiol 50: 1561-1569, 2007.

114. Saris WH, Goodpaster BH, Jeukendrup AE, Brouns F, Halliday D, and Wagenmakers AJ. Exogenous carbohydrate oxidation from different carbohydrate sources during exercise. F Appl Physiol 75: 2168-2172, 1993.

115. Sawka MN, Burke LM, Eichner ER, Maughan RJ, Montain SJ, and Stachenfeld NS. American College of Sports Medicine position stand. Exercise and fluid replacement. Medicine and science in sports and exercise 39: 377-390, 2007.

116. Schwartz AE, Vanagunas A, and Kamel PL. Endoscopy to evaluate gastrointestinal bleeding in marathon runners. Ann Intern Med 113: 632-633, 1990.

117. Shiva S, and Gladwin MT. Nitrite mediates cytoprotection after ischemia/reperfusion by modulating mitochondrial function. Basic Res Cardiol 104: 113-119, 2009.

118. Sieber C, Beglinger C, Jaeger K, Hildebrand P, and Stalder GA. Regulation of postprandial mesenteric blood flow in humans: evidence for a cholinergic nervous reflex. Gut 32: 361-366, 1991.

119. Skeik N, Cooper LT, Duncan AA, and Jabr FI. Median arcuate ligament syndrome: a nonvascular, vascular diagnosis. Vasc Endovascular Surg 45: 433-437, 2011.

120. Snyder AC. Overtraining and glycogen depletion hypothesis. Medicine and science in sports and exercise 30: 11461150, 1998.

121. Soffer EE, Summers RW, and Gisolfi C. Effect of exercise on intestinal motility and transit in trained athletes. Am f Physiol 260: G698-702, 1991.

122. Steinbakk M, Naess-Andresen CF, Lingaas E, Dale I, Brandtzaeg P, and Fagerhol MK. Antimicrobial actions of calcium binding leucocyte L1 protein, calprotectin. Lancet 336: 763-765, 1990.

123. Striz I, and Trebichavsky I. Calprotectin - a pleiotropic molecule in acute and chronic inflammation. Physiol Res 53: 245-253, 2004.

124. Ter Steege RW, Geelkerken RH, Huisman AB, and Kolkman JJ. Abdominal symptoms during physical exercise and the role of gastrointestinal ischaemia: a study in 12 symptomatic athletes. Br F Sports Med 2011.

125. ter Steege RW,Van der Palen J, and Kolkman JJ. Prevalence of gastrointestinal complaints in runners competing in a long-distance run: an internet-based observational study in 1281 subjects. Scand J Gastroenterol 43: 1477-1482, 2008.

126. Thuijls G, de Haan JJ, Derikx JP, Daissormont I, Hadfoune M, Heineman E, and Buurman WA. Intestinal cytoskeleton degradation precedes tight junction loss following hemorrhagic shock. Shock 31: 164-169, 2009.

127. Thuijls G, Derikx JP, de Haan JJ, Grootjans J, de Bruine A, Masclee AA, Heineman E, and Buurman WA. Urine-based detection of intestinal tight junction loss. Journal of clinical gastroenterology 44: e14-19, 2010.

128. Thuijls G, van Wijck K, Grootjans J, Derikx JP, van Bijnen AA, Heineman E, Dejong CH, Buurman WA, and Poeze M. Early diagnosis of intestinal ischemia using urinary and plasma fatty acid binding proteins. Annals of surgery 253: 303-308, 2011.

129. Tibble J, Sigthorsson G, Foster R, Sherwood R, Fagerhol M, and Bjarnason I. Faecal calprotectin and faecal occult blood tests in the diagnosis of colorectal carcinoma and adenoma. Gut 49: 402-408, 2001.

130. Vaishnava S, Behrendt CL, Ismail AS, Eckmann L, and Hooper LV. Paneth cells directly sense Gut commensals and maintain homeostasis at the intestinal host-microbial interface. Proc Natl Acad Sci U S A 105: 2085820863, 2008.

131. van de Poll MC, Ligthart-Melis GC, Boelens PG, Deutz NE, van Leeuwen PA, and Dejong CH. Intestinal and hepatic metabolism of glutamine and citrulline in humans. The fournal of physiology 581: 819-827, 2007.

132. van Nieuwenhoven MA, Brouns F, and Brummer RJ. The effect of physical exercise on parameters of gastrointestinal function. Neurogastroenterol Motil 11: 431-439, 1999.

133. van Nieuwenhoven MA, Brouns F, and Brummer RJ. Gastrointestinal profile of symptomatic athletes at rest and during physical exercise. European journal of applied physiology 91: 429-434, 2004.

134. Van Noord D, Sana A, Benaron DA, Pattynama PM, Verhagen HJ, Hansen BE, Kuipers EJ, and Mensink PB. Endoscopic visible light spectroscopy: a new, minimally invasive technique to diagnose chronic GI ischemia. Gastrointest Endosc 73: 291-298, 2011. 
135. van Wijck K, Lenaerts K, van Loon LJ, Peters WH, Buurman WA, and Dejong CH. Exercise-induced splanchnic hypoperfusion results in Gut dysfunction in healthy men. PloS ONE 6: e22366, 2011.

136. van Wijck K, van Eijk HM, Buurman WA, Dejong CH, and Lenaerts K. Novel analytical approach to a multisugar whole Gut permeability assay. F Chromatogr B Analyt Technol Biomed Life Sci 879: 2794-2801, 2011.

137. Vanhatalo A, Fulford J, Bailey SJ, Blackwell JR, Winyard PG, and Jones AM. Dietary nitrate reduces muscle metabolic perturbation and improves exercise tolerance in hypoxia. The fournal of physiology 589: 5517-5528, 2011.

138. Vanheel B, Van de Voorde J, and Leusen I. Contribution of nitric oxide to the endothelium-dependent hyperpolarization in rat aorta. The fournal of physiology 475: 277-284, 1994.

139. Verdant CL, De Backer D, Bruhn A, Clausi CM, Su F, Wang Z, Rodriguez H, Pries AR, and Vincent JL. Evaluation of sublingual and Gut mucosal microcirculation in sepsis: a quantitative analysis. Critical care medicine 37: 2875-2881, 2009.

140. Vermeulen MA, van de Poll MC, Ligthart-Melis GC, Dejong CH, van den Tol MP, Boelens PG, and van Leeuwen PA. Specific amino acids in the critically ill patient--exogenous glutamine/arginine: a common denominator? Critical care medicine 35: S568-576, 2007.

141. Wright H, Collins M, Villiers RD, and Schwellnus MP. Are splanchnic hemodynamics related to the development of gastrointestinal symptoms in ironman triathletes? A prospective cohort study. Clin F Sport Med 21: 337-343, 2011.

142. Zacho HD, Kristensen NB, Henriksen JH, and Abrahamsen J. Validation of 99mTechnetium labelled mebrofenin hepatic extraction method to quantify meal induced splanchnic blood flow responses using a porcine model. F Appl Physiol 2011. 


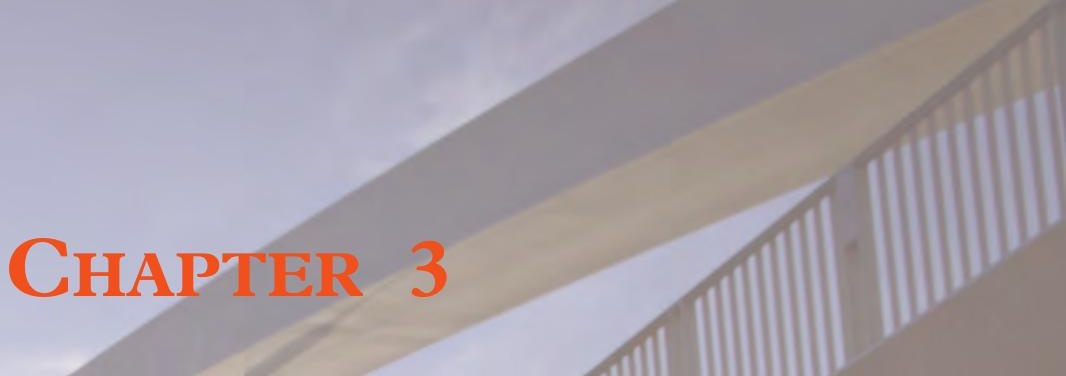

Early diagnosis of intestinal ischemia using urinary and plasma fatty acid binding proteins

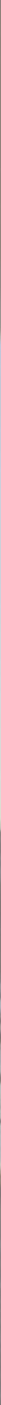




\section{ABSTRACT}

This study aims at improving diagnosis of intestinal ischemia, by measuring plasma and urinary fatty acid binding protein (FABP) levels.

Fifty consecutive patients suspected of intestinal ischemia were included and blood and urine were sampled at time of suspicion. Plasma and urinary concentrations of intestinal FABP (I-FABP), liver FABP (L-FABP), and ileal bile acid binding protein (I-BABP) were measured using enzyme linked immunosorbent assays.

Twenty-two patients suspected of intestinal ischemia were diagnosed with intestinal ischemia, 24 patients were diagnosed with other diseases, and 4 patients were excluded from further analysis fulfilling exclusion criteria. Median plasma concentrations of I-FABP and L-FABP and urinary concentrations of all 3 markers were significantly higher in patients with proven intestinal ischemia than in patients suspected of intestinal ischemia with other final diagnoses (plasma I-FABP; $653 \mathrm{pg} / \mathrm{mL}$ vs. $109 \mathrm{pg} / \mathrm{mL}, P=0.02$, plasma L-FABP; $117 \mathrm{ng} / \mathrm{mL}$ vs. $25 \mathrm{ng} / \mathrm{mL}$, $P=0.006$, urine I-FABP; $3377 \mathrm{pg} / \mathrm{mL}$ vs. $115 \mathrm{pg} / \mathrm{mL}, P=0.001$, urine L-FABP; $1,199 \mathrm{ng} / \mathrm{mL}$ vs. $37 \mathrm{ng} / \mathrm{mL}, P=0.004$, urine I-BABP; $48.6 \mathrm{ng} / \mathrm{mL}$ vs. $0.6 \mathrm{ng} / \mathrm{mL}, P=0.002$ ). Positive and negative likelihood ratios significantly increased positive posttest probability and decreased negative posttest probability on intestinal ischemia. In patients with intestinal ischemia a trend to higher plasma I-BABP levels was observed when the ileum was involved $(18.4 \mathrm{ng} / \mathrm{mL}$ vs. 2.9 $\mathrm{ng} / \mathrm{mL}, P=0.05)$.

In conclusion, plasma and especially urinary I-FABP and L-FABP levels and urinary I-BABP levels can improve early diagnosis of intestinal ischemia. Furthermore, plasma I-BABP levels can help in localizing ileal ischemia. 


\section{INTRODUCTION}

Acute intestinal ischemia is a severe intestinal emergency with high mortality (17). It can be precipitated by a number of pathophysiological processes such as arterial embolism, arterial and venous thrombosis, aortic dissection, intestinal obstruction due to adhesive diseases after previous operations, and non-occlusive disorders (e.g., hypovolemia, hypotension). Clinical diagnosis of intestinal ischemia remains difficult, because of the nonspecific clinical signs in the early phase. Especially, in high-risk patients clinical history taking and physical examination is hampered because of sedation and analgesia. In addition, the lack of appropriate diagnostic tests further hampers correct diagnosis (15). In most patients, intestinal ischemia is recognized only when the patient has extensive transmural necrosis or sepsis. Delayed diagnosis is a major reason for the high-mortality rates of intestinal ischemia, because a diagnostic delay of 24 hours decreases survival rates by more than $20 \%$ (17). Therefore, it is important to improve early diagnosis of intestinal ischemia.

In this context, fatty acid binding proteins (FABPs) are potentially interesting. Fatty acid binding proteins are small cytosolic proteins, which are released upon enterocyte membrane integrity loss. They are readily released into the circulation and renally cleared, which makes them useful as plasma and urine markers for enterocyte damage $(5,9,11)$. Previously, we showed increased plasma FABP levels after induction of intestinal ischemia using a newly developed human, experimental controlled model of 30 minutes of intestinal ischemia followed by variable periods of reperfusion (4). Also following major vascular surgery and in diseases with known intestinal hypoperfusion, such as necrotizing enterocolitis increased plasma and urinary FABP levels were observed $(6,10,14)$. Three FABP isoforms are expressed in the intestine; intestinal fatty acid binding protein (I-FABP), ileal bile acid binding protein (I-BABP) and liver fatty acid binding protein (L-FABP). The presence of I-FABP is limited to mature enterocytes of the small and large intestine $(6,18)$. Ileal bile acid binding protein is solely expressed in mature enterocytes of the ileum (21), whereas L-FABP is expressed in small and large intestine, liver, and kidney epithelial cells $(6,16,18)$.

To investigate the usefulness of FABPs as new diagnostic tests for acute intestinal ischemia the following research questions can be evaluated: Phase 1 question - patients with the target disorder have different test results from normal individuals? Phase 2 question - are patients with certain test results more likely to have the target disorder than individuals with other test results (19)? These phase 1 and 2 questions have been studied by Lieberman et al. (14) and Kanda et al (11). They showed that I-FABP plasma and urine levels are significantly higher in patients with intestinal ischemia than in healthy controls.

The phase 3 question is: do circulating and urinary FABP levels distinguish patients with intestinal ischemia and patients without intestinal ischemia among patients in whom acute intestinal ischemia is suspected? This phase 3 question has been studied in a selected patient population suspected of ischemia due to small bowel obstruction by Cronk et al (3). They showed elevated plasma 
and urine I-FABP levels in 3 of 3 patients with necrosis and 4 of 18 patients without necrosis and concluded that I-FABP is a sensitive marker for ischemia in mechanical small bowel obstruction. Because one of the diagnostic problems of intestinal ischemia is the diversity in pathophysiological processes, we aimed to improve diagnosis of intestinal ischemia no matter of the origin. Therefore, we investigated whether circulating and urinary FABP levels distinguish patients with intestinal ischemia and patients without intestinal ischemia among all patients in whom intestinal ischemia is suspected.

\section{MATERIALS AND METHODS}

\section{Patients}

Following sample size analysis (based on the results of the study of Kanda et al (11), 50 consecutive patients clinically suspected of acute intestinal ischemia by the treating physician were included at the Maastricht University Medical Center from September 2007 to July 2009. This patient group consisted of patients presenting with acute abdominal pain on the emergency department and high-risk in hospital patients. Patients with preexisting intestinal damage due to intestinal surgery within 7 days before suspicion of intestinal ischemia were excluded. The study was approved by the local ethics committee and informed consent was obtained from each participant. After inclusion EDTA plasma samples and urine samples were collected, centrifuged at 4C, $4000 \mathrm{rpm}, 15$ minutes, and stored at $-20^{\circ} \mathrm{C}$ until analysis. Plasma and urine samples were collected immediately at suspicion of intestinal ischemia, and at least 1 hour after suspicion of ischemia. For this reason, urine samples could only be collected from 19 patients. Diagnosis was based on findings during surgery or autopsy and verified by histopathological examination of the intestine.

In case no surgery or autopsy was available for diagnosis, final diagnosis was determined by consensus among 2 of the authors (G.T. and M.P.) without prior knowledge of plasma and urine FABP values. In all patients diagnosed with intestinal ischemia, diagnosis was objectively made. Also, in the patient group with other diagnosis, there was no diagnostic difficulty because of clear diagnosis in most cases. Only in 4 patients the ultimate diagnosis was unknown, because of complete recovery after conservative management we agreed that no clinically relevant ischemia occurred.

\section{Conventional Markers for Intestinal Ischemia}

To compare the studied markers with the classical markers to diagnose intestinal ischemia, plasma lactate level, leukocyte count, and base excess, determined as routine patient care in the first blood sample upon suspicion of acute intestinal ischemia by the clinical chemistry laboratory, were analyzed. Blood samples of these reference tests are taken at the same time-point as the samples for FABP measurement. 


\section{Urinary and Plasma FABP Measurement}

Plasma and urinary I-FABP and L-FABP concentrations were determined using standard enzyme linked immunosorbent assays (Hycult Biotech, Uden, the Netherlands) and I-BABP concentration was determined as previously described.16 The detection limit for IFABP, L-FABP, and I-BABP was $40 \mathrm{pg} / \mathrm{mL}, 2 \mathrm{ng} / \mathrm{mL}$ and $0.25 \mathrm{ng} / \mathrm{mL}$, respectively. Human FABP enzyme linked immunosorbent assays kits are solid-phase enzyme-linked immunosorbent assays based on the sandwich principle with a working time of $31 / 2$ hours.

\section{Statistics}

Statistical analysis was performed with GraphPad Prism 4 for Windows (GraphPad Software Inc., San Diego, CA). Normality of all data obtained was evaluated by Kolmogorov-Smirnov test. Mann-Whitney $U$ test was used for between group comparisons. Data are presented as median and range. Sex, number of patients (unnecessary) operated, and number of patients died was compared using Fisher exact test. To find the cutoff points of plasma and urinary FABP levels that most accurately discriminated patients with intestinal ischemia and patients with other final diagnoses, receiver operating characteristics (ROC) curves were drawn by plotting sensitivity against 1-specificity for all thresholds. Furthermore, a ROC curve was drawn for plasma I-BABP to find the cutoff point that most accurately discriminated patients with ileal ischemia and patients with intestinal ischemia without ileal involvement. Overall accuracy of the markers was summarized using area under the curve. The best cutoff point was defined as the maximum sum of sensitivity and specificity. Cutoff points were used to calculate sensitivity, specificity, positive and negative likelihood ratios, and positive and negative posttest probabilities. $P<0.05$ was considered statistically significant. Standards for Reporting of Diagnostic Accuracy statement for reporting studies of diagnostic accuracy was used in this study (2).

\section{RESULTS}

\section{Patients suspected of intestinal ischemia}

A total of 50 patients were enrolled is this study as scheduled. In 22 patients suspected of intestinal ischemia, diagnosis was confirmed by histopathology. Twenty-four patients were diagnosed with other final diagnosis; coprostasis $(n=2)$, pancreatitis $(n=2)$, diverticulitis $(n=4)$, cholecystitis $(n=3)$, ileus $(n=1)$, intestinal stenosis $(n=1)$, concealed perforation $(n=2)$, urosepsis $(n=1)$, perforated appendicitis $(n=1)$, retroperitoneal bleeding of unknown origin $(n=1)$, stomach perforation $(n=1)$, small intestinal herniation $(n=1)$, gastrointestinal problem of unknown origin $(n=4)$. Four patients were not included in the analyses because of preexisting intestinal damage due to a preceding colon operation (Table 1). 
Table 1: Baseline characteristics of patients with intestinal ischemia or other diagnoses.

\begin{tabular}{|c|c|c|c|}
\hline & Intestinal ischemia & Other diagnoses & $P$ value \\
\hline Group size (no. of patients) & 22 & 24 & \\
\hline Age (years) & $69(11-84)$ & $67(42-86)$ & 0.62 \\
\hline Sex $(M: V)$ & $8: 14$ & $10: 14$ & 0.77 \\
\hline Operated & 21 & 13 & 0.002 \\
\hline Unnecessarily operated & 0 & 5 & 0.005 \\
\hline Died & 7 & 0 & 0.003 \\
\hline Time interval between sampling & $5 \mathrm{~h}$ & $10 \mathrm{~h}$ & 0.32 \\
\hline and operation & $(0-42 \mathrm{~h})$ & $(0-23 h)$ & 0.003 \\
\hline
\end{tabular}

Data are presented as median (range).

\section{Conventional blood laboratory tests}

Laboratory tests currently in use to aid in the diagnosis intestinal ischemia are lactate, leukocyte count, and base excess. These markers were not able to discriminate patients with intestinal ischemia and patients with other diseases (lactate; $2.5 \mathrm{mmol} / \mathrm{L}$ [0.4-23.1] vs. $2.3 \mathrm{mmol} / \mathrm{L}$ [1.05.2], $P=0.56$, leukocyte count; $13.9 \mathrm{mg} / \mathrm{L}[1.7-28.0]$ vs. $12.7 \mathrm{mg} / \mathrm{L}[3.3-33.7], P=0.89$, base excess; -3.0 mmol/L [-15.9-3.9] vs. $-3.7 \mathrm{mmol} / \mathrm{L}$ [-6.9-2.5], $P=0.60$ ) (Figure 1).

\section{FABPs for diagnosis of intestinal ischemia}

Median plasma I-FABP and L-FABP levels were significantly higher in patients with proven intestinal ischemia than in patients with other diagnoses (I-FABP; $653 \mathrm{pg} / \mathrm{mL}$ [40-74,711] vs. $109 \mathrm{pg} / \mathrm{mL}$ [40-1,691], $P=0.02$ and L-FABP; $117 \mathrm{ng} / \mathrm{mL}$ [6-37,422] vs. $25 \mathrm{ng} / \mathrm{mL}$ [5-419], $P=0.006$ ) (Figure 2). Plasma I-BABP levels were not able to discriminate patients with ischemia and patients with other diseases $(14.5 \mathrm{ng} / \mathrm{mL}$ [0.3-616.6] vs. $4.9 \mathrm{ng} / \mathrm{mL}$ [0.3-305.2], $P=0.15$ ) (Figure 2). In 19 patients of whom urine was collected at the moment of suspicion of intestinal ischemia, urinary levels of all 3 markers were significantly higher in patients with intestinal ischemia $(\mathrm{n}=10)$ than in patients with other diagnoses $(\mathrm{n}=9)(\mathrm{I}-\mathrm{FABP} ; 3,377 \mathrm{pg} / \mathrm{mL}$ [142442,795] vs. $115 \mathrm{pg} / \mathrm{mL}$ [40-1,620], $P=0.0006$, L-FABP; $1,199 \mathrm{ng} / \mathrm{mL}[138-39,176]$ vs. 37 $\mathrm{ng} / \mathrm{mL}$ [2-536], $\mathrm{P}=0.004$, IBABP; $48.6 \mathrm{ng} / \mathrm{mL}$ [1.8-900] vs. $0.6 \mathrm{ng} / \mathrm{mL}$ [0.25-38.9], $P=0.002$ ) (Figure 3). Ideal cutoff points to discriminate patients with intestinal ischemia and patients without intestinal ischemia among patients suspected of intestinal ischemia were assessed as maximum sum of sensitivity and specificity using ROC curves. Ideal cutoff points, sensitivity, specificity, and area under the ROC curve are depicted in Table 2. 

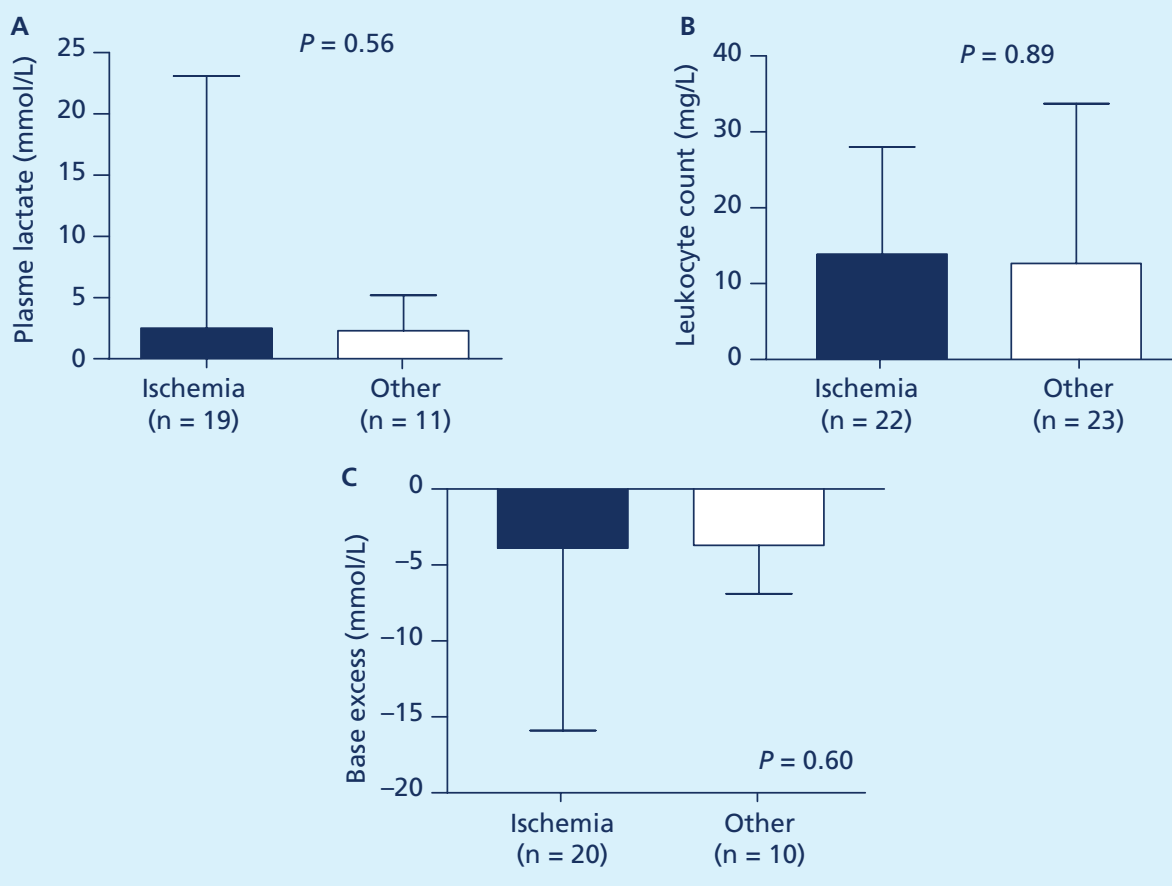

Figure 1: No conventional marker did differ between patients with intestinal ischemia an patients with other final diagnoses A) Median plasma lactate levels; B) blood leukocyte count; C) base excess. Data are reported as median and range.

\section{Accuracy of FABPs in diagnosing intestinal ischemia}

Diagnostic accuracy of plasma and urinary FABPs is represented by the probability on intestinal ischemia after performing the test. Posttest probabilities are determined using pretest probability and likelihood ratios. The pretest probability on intestinal ischemia in our patient population was $48 \%$, because 22 of 46 patients suspected of intestinal ischemia were ultimately diagnosed with intestinal ischemia. In the patients of whom urine was sampled the pretest probability on intestinal ischemia was $53 \%$, given that 10 of 19 patients were ultimately diagnosed with ischemia. Detection of plasma I-FABP and L-FABP levels above their ideal cutoff points resulted in an increase of disease probability to $68 \%(52-81)$ and $82 \%$ (59-93) respectively. Urinary IFABP, L-FABP and I-BABP levels above their determined cutoff points increased the chance on disease to respectively $90 \%$ (58-81), 80\% (53-94), and 88\% (51-98). Test results below the cutoff point, that is, negative test results, decreased the chance on disease. Negative test results for plasma I-FABP and LFABP resulted in a likelihood of having the disease of 29\% (18-45) and 30\% (21-42). Urinary I-FABP, L-FABP, and I-BABP levels below their cutoff point resulted in a likelihood of having the disease of respectively $11 \%(2-45), 23 \%(7-51)$, and $28 \%(13-50)$. Combining the described markers did not improve diagnostic accuracy because of large 

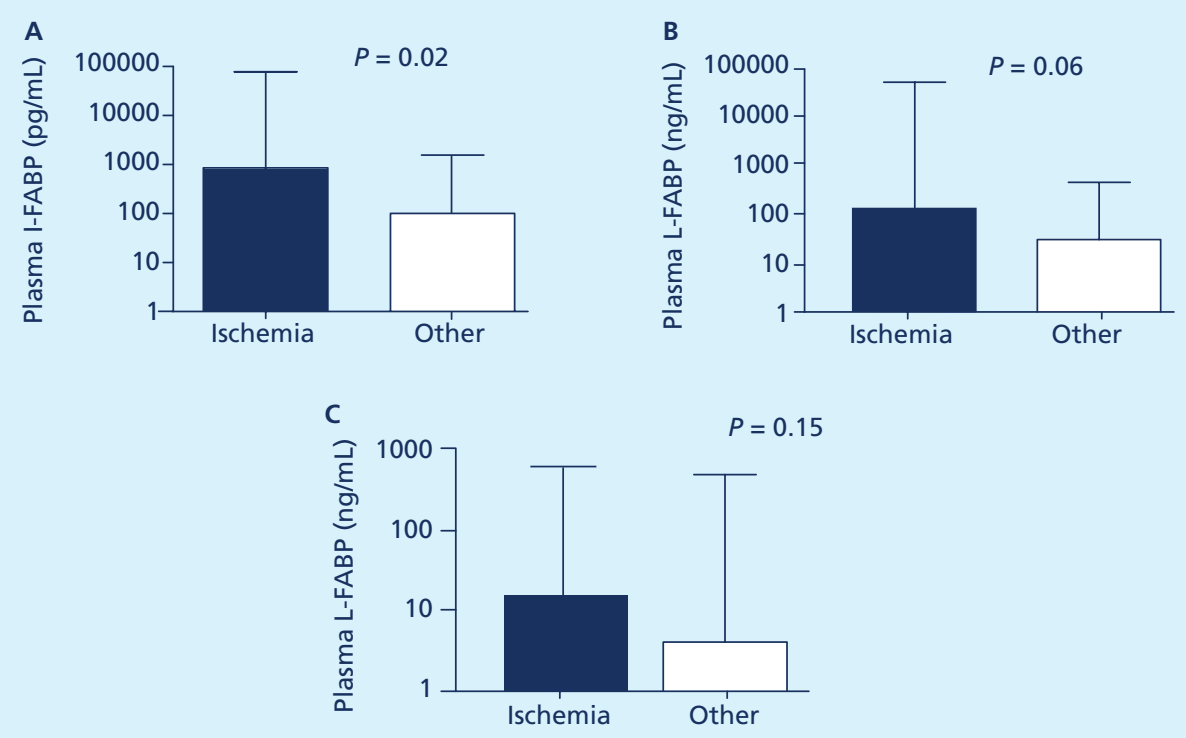

Figure 2: Plasma I-FABP and L-FABP concentrations were significantly higher in patients with intestinal ischemia $(n=22)$ than in patients with other final diagnoses $(n=24)$.

A) Plasma I-FABP; B) plasma L-FABP; C) plasma I-BABP concentrations. Data are reported as median and range.

colinearity of the markers (data not shown). The clinical accuracy of all markers reflected by posttest probability and their corresponding pretest probabilities and likelihood ratios are depicted in Table 3.

\section{Localizing ischemia using plasma I-BABP levels}

Because I-BABP is solely expressed in the ileum, we investigated whether increased plasma I-BABP levels can be used as marker for ileal ischemia. Indeed, within the patient group with intestinal ischemia median plasma I-BABP levels showed a clear trend to be increased in patients with ileal ischemia compared with patients without ileal involvement $(18.4 \mathrm{ng} / \mathrm{mL}(0.4-616.6)$ vs. $2.9 \mathrm{ng} / \mathrm{mL}(0.3-51.5), P=0.05)$ (Figure 4). The ideal cutoff point to discriminate patients with ileal involvement and patients without ileal involvement was $12.2 \mathrm{ng} / \mathrm{mL}$, with a sensitivity of $69 \%$, and a specificity of $83 \%$. The pretest probability of ileal involvement in patients with intestinal ischemia was $73 \%$. Posttest disease probability of a positive test result was $92 \%$ (6499) and the likelihood of having the disease when test result was negative was $50 \%$ (31-69). The current study could not be used to determine whether increased urinary I-BABP levels indicate ileal ischemia, due to lack of appropriate sample size. 

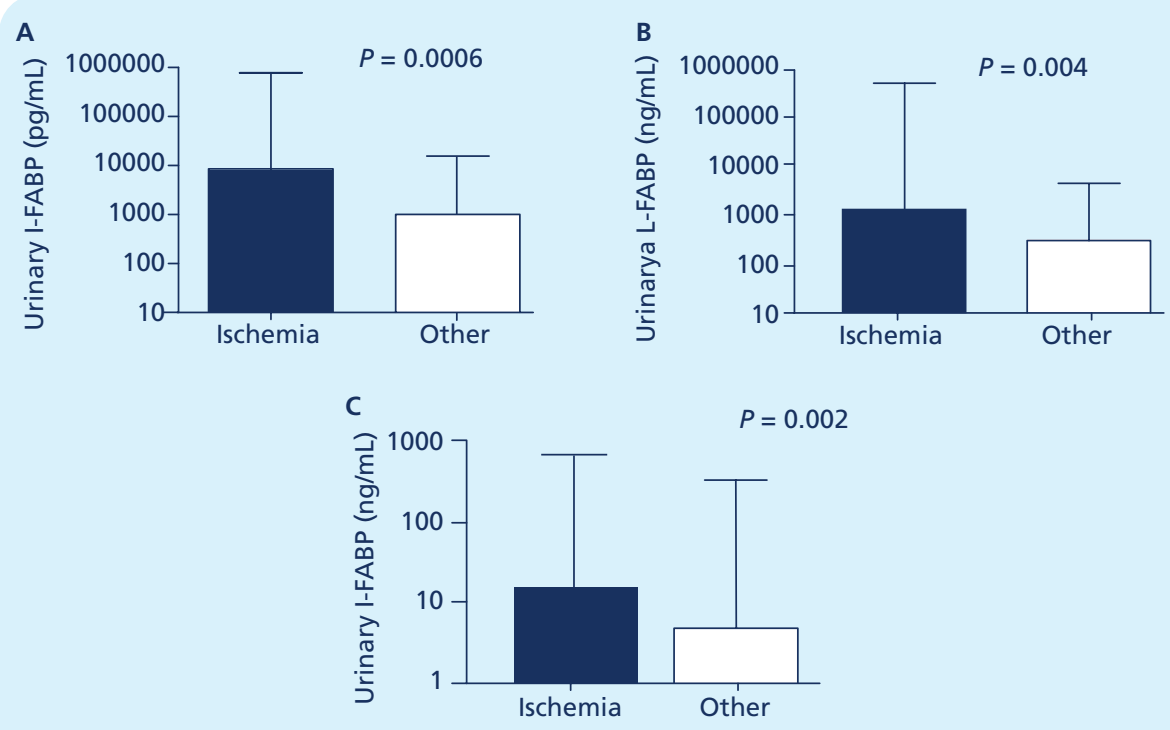

Figure 3: Urinary I-FABP $(A), L-F A B P(B)$ and I-BABP $(C)$ concentrations were significantly higher in patients with intestinal ischemia $(n=10)$ than in patients with other final diagnoses $(n=9)$

Data are reported as median and range.

Table 2: Overall accuracy ${ }^{1}$ to differentiate patients with intestinal ischemia or other diagnoses.

\begin{tabular}{|c|c|c|c|c|c|}
\hline Marker & Cutoff point & Sensitivity & Specificity & $\begin{array}{l}\text { Area under the curve } \\
\qquad(95 \% \mathrm{CI})\end{array}$ & $P$ value \\
\hline Plasma I-FABP & $268 \mathrm{pg} / \mathrm{ml}$ & 0.68 & 0.71 & $0.70(0.53-0.86)$ & 0.02 \\
\hline Urine I-FABP & $551 \mathrm{pg} / \mathrm{ml}$ & 0.90 & 0.89 & $0.93(0.82-1.04)$ & 0.001 \\
\hline Plasma L-FABP & $78 \mathrm{ng} / \mathrm{ml}$ & 0.69 & 0.88 & $0.74(0.59-0.88)$ & 0.006 \\
\hline Urine L-FABP & $180 \mathrm{ng} / \mathrm{ml}$ & 0.80 & 0.78 & $0.88(0.72-1.03)$ & 0.006 \\
\hline Plasma I-BABP & $7 \mathrm{ng} / \mathrm{ml}$ & 0.64 & 0.63 & $0.63(0.45-0.80)$ & 0.15 \\
\hline Urine I-BABP & $5 \mathrm{ng} / \mathrm{ml}$ & 0.70 & 0.89 & $0.91(0.78-1.05)$ & 0.003 \\
\hline
\end{tabular}

${ }^{1}$ Area under the curve and cutoff points, defined as the maximum sum of sensitivity and specificity, using receiver operating characteristics curves, with corresponding sensitivity and specificity of fatty acid binding proteins (FABPs) to differentiate patients with intestinal ischemia and patients without intestinal ischemia. 
Table 3: Likelihood ratios and pre- and posttest probabilities of FABPs

\begin{tabular}{|lccccc}
\hline Marker & $\begin{array}{c}\text { Positive } \\
\text { likelihood } \\
\text { ratio }(95 \% \text { CI })\end{array}$ & $\begin{array}{c}\text { Negative } \\
\text { likelihood } \\
\text { ratio }(95 \% \text { CI })\end{array}$ & $\begin{array}{c}\text { Potential } \\
\text { probability } \\
(\%)\end{array}$ & $\begin{array}{c}\text { Positive test } \\
\text { posterior } \\
\text { probability } \\
(95 \% \text { CI })\end{array}$ & $\begin{array}{c}\text { Negative test } \\
\text { posterior } \\
\text { probability } \\
(95 \% \text { CI })\end{array}$ \\
\hline Plasma I-FABP & $2.34(1.18-4.67)$ & $0.45(0.23-0.87)$ & 48 & $68(52-81)$ & $29(18-45)$ \\
Urine I-FABP & $8.18(1.25-52.0)$ & $0.11(0.02-0.73)$ & 53 & $90(58-98)$ & $11(2-45)$ \\
Plasma L-FABP & $4.92(1.57-15.0)$ & $0.47(0.28-0.79)$ & 48 & $82(59-93)$ & $30(21-42)$ \\
Urine L-FABP & $3.64(1.02-13.0)$ & $0.26(0.07-0.93)$ & 53 & $80(53-94)$ & $23(7-51)$ \\
Plasma I-BABP & $1.70(0.93-3.11)$ & $0.58(0.31-1.10)$ & 48 & $61(46-74)$ & $35(22-50)$ \\
Urine I-BABP & $6.31(0.94-42.0)$ & $0.34(0.13-0.89)$ & 53 & $88(51-98)$ & $28(13-50)$ \\
\hline
\end{tabular}

CI, confidence interval

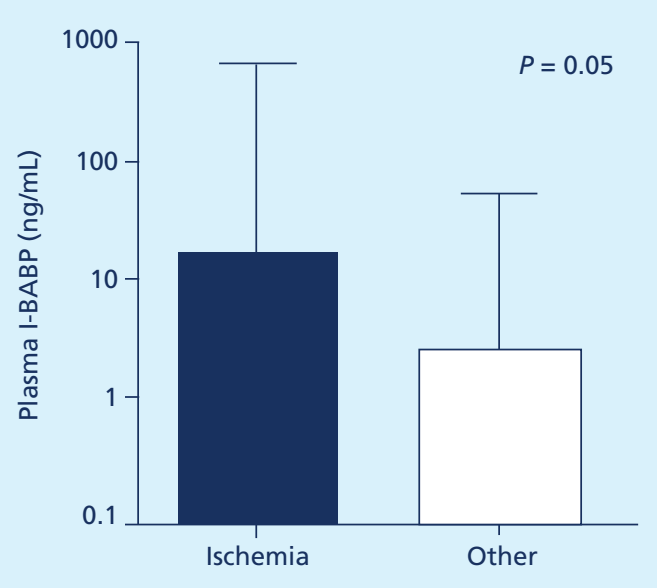

Figure 4: Plasma I-BABP concentrations were significantly higher in patients with ileal ischemia (n=16) than in patients with intestinal ischemia without ileal involvement $(n=9)$

Data are reported as median and range.

\section{DISCUSSION}

Early and correct diagnosis and treatment of intestinal ischemia is crucial to reduce mortality (12). A high suspicion after patient history and physical examination serves as the cornerstone to early diagnose mesenteric ischemia. However, discrepancy often exists between the signs and symptoms during clinical examination, leading to diagnostic uncertainty. Next to the clinical features leading to the suspicion of intestinal ischemia, also laboratory tests are used to improve the diagnostic accuracy of intestinal ischemia. Currently used laboratory tests are mainly plasma lactate, blood leukocyte count and base excess. In accordance with previous studies, we show 
that these 3 markers do not allow differentiation of our patients with intestinal ischemia and patients without intestinal ischemia $(7,17)$.

It could be argued that imaging modalities can be used to improve diagnosis. However, the value of current (radiological) investigations to diagnose intestinal ischemia is limited because of the diversity of causes leading to intestinal ischemia $(1,8,13)$. Accordingly, the need for other (plasma and urine) markers to diagnose intestinal ischemia early remains high. Evennett et al. (7) recently reviewed studies on serological markers for diagnosing intestinal ischemia. They concluded that novel markers for intestinal ischemia such as D-lactate, Glutathione S-transferase (GST), and IFABP may offer improved diagnostic accuracy, although the number of studies evaluating novel markers for intestinal ischemia is limited. Previous studies showed that plasma FABP levels were able to discriminate patients with intestinal ischemia and healthy controls $(11,14)$.

Because one of the diagnostic problems of intestinal ischemia is the diversity in pathophysiological processes, we aimed to improve diagnosis of intestinal ischemia no matter of the origin. Therefore, we investigated whether plasma and urinary FABP levels were able to distinguish patients with intestinal ischemia from patients without intestinal ischemia without differentiation in underlying pathophysiological process.

Plasma and urinary I-FABP and L-FABP levels and urinary I-BABP levels were significantly higher in patients with intestinal ischemia than in patients with other final diseases. Although measurement of plasma FABP levels was shown to increase the posttest probability on intestinal ischemia only minimally, detection of urinary FABP levels resulted in markedly increased positive posttest probabilities and clearly decreased negative posttest probabilities. The rapid clearance of plasma FABPs (half-life time of 11 minutes) into urine and urinary FABP accumulation reflecting intestinal damage over a period of time could explain the higher diagnostic accuracy of urinary FABP levels than plasma levels of the same marker (20). Also studies investigating the value of FABPs in diagnosing intestinal ischemia with specific underlying pathophysiology are useful, because especially in high-risk patients with bowel obstruction due to adhesive disease, unnecessary or delayed operation with perforation should be avoided. Cronk et al. showed that urinary and plasma FABP are elevated in this selected patient group (3).

In this study, we determined optimum cutoff points for all markers described, represented by the maximum sum of sensitivity and specificity of the marker. Instead of using an optimum cutoff point, also cutoff points can be applied with maximum sensitivity at the expense of specificity or vice versa. Because intestinal ischemia is a severe disease with high morbidity and mortality, which steeply increase if operation is delayed, a diagnostic test with high sensitivity is desirable. However, a resultant decrease in specificity can lead to unnecessary morbidity due to a surgical procedure that may ultimately prove negative for intestinal ischemia. This taken into account, we considered the cutoff points represented by the sum of maximum sensitivity and specificity as best cutoff point to differentiate in patients with and without intestinal ischemia in a population of suspected ischemia. Furthermore, these clinical tests should be used as a sup- 
plement to patient history and physical examination, because plasma and urinary levels above/below a predetermined cutoff point increase/decrease the likelihood of having a disease but are not conclusive. This is especially true for levels closely approaching their cutoff point. High plasma and urinary FABP levels at the moment of suspicion may justify early operation, whereas low levels can be the reason for a conservative policy guided by clinical disease course, thereby reducing the rate of unnecessary surgical interventions.

Tests with high diagnostic accuracy greatly contribute to a correct diagnosis of disease. The described markers improve diagnostic accuracy markedly. To further improve diagnostic accuracy we investigated if combining the markers yielded better test results. Unfortunately, combining the described markers did not improve diagnostic accuracy due to large colinearity of the markers.

Finally, we investigated if plasma I-BABP levels could differentiate in patients with ileal ischemia and patients with intestinal ischemia without ileal involvement. We observed a trend to increased I-BABP levels in patients with ileal involvement. Establishing the localization of ischemia could facilitate the operative procedure.

In this study, samples were prospectively collected if there was a clinical suspicion of intestinal ischemia and analyzed after completion of patient inclusion. The median time between sampling and operation in patients with intestinal ischemia was 5 hours 44 minutes ( $0-42 \mathrm{~h}$ ), indicating that diagnosis and consequently treatment can be earlier following measurement of FABP. However, future analyses should and can be performed at the moment of suspicion of intestinal ischemia to be of clinical use and to evaluate whether doing these tests results in better patients outcome.

In conclusion, plasma and urinary I-FABP and L-FABP levels and urinary I-BABP levels were significantly increased at the moment of suspicion of intestinal ischemia in patients with proven intestinal ischemia compared with patients with other final diagnoses. Although plasma FABP levels only minimally increased posttest probability on intestinal ischemia, measurement of urinary FABP levels resulted in markedly increased positive posttest probabilities and clearly decreased negative posttest probabilities. Therefore, urinary levels of these markers can improve early diagnosis of intestinal ischemia and can prevent a delay in surgical intervention. Furthermore, plasma I-BABP levels may help localizing ileal ischemia. 


\section{REFERENCES}

1. Angelelli G, Scardapane A, Memeo M, Stabile Ianora AA, and Rotondo A. Acute bowel ischemia: CT findings. Eur F Radiol 50: 37-47, 2004.

2. Bossuyt PM, Reitsma JB, Bruns DE, Gatsonis CA, Glasziou PP, Irwig LM, Moher D, Rennie D, de Vet HC, and Lijmer JG. The STARD statement for reporting studies of diagnostic accuracy: explanation and elaboration. The Standards for Reporting of Diagnostic Accuracy Group. Croat Med F 44: 639-650, 2003.

3. Cronk DR, Houseworth TP, Cuadrado DG, Herbert GS, McNutt PM, and Azarow KS. Intestinal fatty acid binding protein (I-FABP) for the detection of strangulated mechanical small bowel obstruction. Curr Surg 63: 322-325, 2006.

4. Derikx JP, Matthijsen RA, de Bruine AP, van Bijnen AA, Heineman E, van Dam RM, Dejong CH, and Buurman WA. Rapid reversal of human intestinal ischemia-reperfusion induced damage by shedding of injured enterocytes and reepithelialisation. PloS ONE 3: e3428, 2008.

5. Derikx JP, Poeze M, van Bijnen AA, Buurman WA, and Heineman E. Evidence for intestinal and liver epithelial cell injury in the early phase of sepsis. Shock 28: 544-548, 2007.

6. Derikx JP,Vreugdenhil AC, Van den Neucker AM, Grootjans J, van Bijnen AA, Damoiseaux JG, van Heurn LW, Heineman E, and Buurman WA. A pilot study on the noninvasive evaluation of intestinal damage in celiac disease using I-FABP and L-FABP. Fournal of clinical gastroenterology 43: 727-733, 2009.

7. Evennett NJ, Petrov MS, Mittal A, and Windsor JA. Systematic review and pooled estimates for the diagnostic accuracy of serological markers for intestinal ischemia. World journal of surgery 33: 1374-1383, 2009.

8. Furukawa A, Kanasaki S, Kono N, Wakamiya M, Tanaka T, Takahashi M, and Murata K. CT diagnosis of acute mesenteric ischemia from various causes. AfR Am f Roentgenol 192: 408-416, 2009.

9. Gollin G, Marks C, and Marks WH. Intestinal fatty acid binding protein in serum and urine reflects early ischemic injury to the small bowel. Surgery 113: 545-551, 1993.

10. Hanssen SJ, Derikx JP, Vermeulen Windsant IC, Heijmans JH, Koeppel TA, Schurink GW, Buurman WA, and Jacobs MJ. Visceral injury and systemic inflammation in patients undergoing extracorporeal circulation during aortic surgery. Annals of surgery 248: 117-125, 2008.

11. Kanda T, Fujii H, Tani T, Murakami H, Suda T, SakaiY, Ono T, and Hatakeyama K. Intestinal fatty acid-binding protein is a useful diagnostic marker for mesenteric infarction in humans. Gastroenterology 110: 339-343, 1996.

12. Kougias P, Lau D, El Sayed HF, Zhou W, Huynh TT, and Lin PH. Determinants of mortality and treatment outcome following surgical interventions for acute mesenteric ischemia. F Vasc Surg 46: 467-474, 2007.

13. Kozuch PL, and Brandt LJ. Review article: diagnosis and management of mesenteric ischaemia with an emphasis on pharmacotherapy. Alimentary pharmacology \& therapeutics 21: 201-215, 2005.

14. Lieberman JM, Sacchettini J, Marks C, and Marks WH. Human intestinal fatty acid binding protein: report of an assay with studies in normal volunteers and intestinal ischemia. Surgery 121: 335-342, 1997.

15.Lock G. Acute intestinal ischaemia. Best Pract Res Clin Gastroenterol 15: 83-98, 2001.

16. Maatman RG, van de Westerlo EM, van Kuppevelt TH, and Veerkamp JH. Molecular identification of the liverand the heart-type fatty acid-binding proteins in human and rat kidney. Use of the reverse transcriptase polymerase chain reaction. The Biochemical journal 288 (Pt 1): 285-290, 1992.

17. Oldenburg WA, Lau LL, Rodenberg TJ, Edmonds HJ, and Burger CD. Acute mesenteric ischemia: a clinical review. Archives of internal medicine 164: 1054-1062, 2004.

18. Pelsers MM, Namiot Z, Kisielewski W, Namiot A, Januszkiewicz M, Hermens WT, and Glatz JF. Intestinal-type and liver-type fatty acid-binding protein in the intestine. Tissue distribution and clinical utility. Clinical biochemistry 36: 529-535, 2003.

19. Sackett DL, and Haynes RB. The architecture of diagnostic research. BMJ 324: 539-541, 2002.

20. van de Poll MC, Derikx JP, Buurman WA, Peters WH, Roelofs HM, Wigmore SJ, and Dejong CH. Liver manipulation causes hepatocyte injury and precedes systemic inflammation in patients undergoing liver resection. World journal of surgery 31: 2033-2038, 2007.

21. Watanabe K, Hoshi N, Tsuura Y, Kanda T, Fujita M, Fujii H, Ono T, and Suzuki T. Immunohistochemical distribution of intestinal $15 \mathrm{kDa}$ protein in human tissues. Archives of histology and cytology 58: 303-306, 1995. 


\section{ABSTRACT}

Increased intestinal permeability is an important measure of disease activity and prognosis. Currently, many permeability tests are available and no consensus has been reached as to which test is best suited. The aim of this study was to compare urinary probe excretion and accuracy of a polyethylene glycol (PEG) assay and dual sugar assay in a double-blinded crossover study to evaluate probe excretion and accuracy of both tests.

Gastrointestinal (GI) permeability was measured in nine volunteers using PEG 400, PEG 1500 and PEG 3350 or lactulose-rhamnose. On four separate days, permeability was analyzed after oral intake of placebo or indomethacin, a drug known to increase intestinal permeability. Plasma intestinal fatty acid binding protein (I-FABP) and calprotectin were determined to verify compromised intestinal integrity after indomethacin consumption. Urinary samples were collected at baseline, hourly up to 5 hours after probe intake, and between 5-24 hours. Urinary excretion of PEG and sugars was determined using HPLC-ELSD and LC-MS, respectively. I ndomethacin intake increased plasma I-FABP and calprotectin levels, reflecting loss of intestinal integrity and inflammation. In this state of indomethacin-induced GI compromise, urinary excretion of the three PEG probes and lactulose increased compared to the placebo situation. Urinary PEG 400 excretion, PEG 3350/PEG 400 ratio, and lactulose/rhamnose ratio could accurately detect indomethacin-induced increases in GI permeability, especially within two hours after probe intake.

In conclusion, hourly urinary excretion and diagnostic accuracy of PEG and sugar probes show high resemblance for detection of indomethacin-induced increases in GI permeability. This comparative study improves our knowledge of permeability analysis in man by providing a clear overview of both tests and demonstrates equivalent performance in the current setting. 


\section{INTRODUCTION}

Under physiological conditions, the gastrointestinal (GI) epithelium provides an effective barrier between the internal and external environment, protecting the body from potentially harmful luminal substances such as bacterial products, digestive enzymes and antigens (3). Loss of GI barrier integrity is accompanied by an increase in epithelial permeability, reflecting a state in which luminal substances can permeate the barrier and enter the systemic circulation, where they may contribute to a systemic inflammatory response and organ dysfunction $(11,16)$. Increased GI permeability plays a role in the etiology and worsening of various intestinal and systemic diseases $(1,18,19,38)$, and is considered an important measure of disease activity and prognosis (3).

Analysis of GI permeability is based on the appearance of orally administered probes such as sugars, ${ }^{51}$ Chromium-labelled EDTA $\left({ }^{51} \mathrm{CrEDTA}\right)$, and polyethylene glycol (PEG), in the circulation and urine after permeation of the intestinal epithelium. Each probe has its specific advantages and disadvantages, and requires a specific method of detection (3).

Radioactive ${ }^{51} \mathrm{CrEDTA}$ is easily detectable as it is not naturally present in man. The commonly applied dose of $100 \mu \mathrm{Ci}(3.7 \mathrm{Mbq}){ }^{51} \mathrm{CrEDTA}$ is similar to the burden of other clinical nuclear diagnostic tests, and its estimated total radiation dose of $0.12 \mathrm{mSv}$ (13) is small compared to the worldwide annual background radiation exposure of approximately $3 \mathrm{mSv}$ (37). However, ${ }^{51}$ CrEDTA-based permeability tests do expose patients to additional radiation. Therefore, the use of ${ }^{51}$ CrEDTA should be avoided in pediatric patients, women of childbearing age, and in patients requiring multiple permeability analyses. Another concern is that the ${ }^{51} \mathrm{CrEDTA}$ assay is the only test that uses a single probe for permeability analysis; therefore, no correction for the influence of individually determined factors such as intestinal transit is performed.

Sugars provide the opportunity to obtain site-specific information on GI permeability when applied in a multiple-sugar test solution $(3,36)$. Classically, the dual sugar (DS) test is used for intestinal permeability analysis. The DS test combines the disaccharide lactulose (MW $342 \mathrm{Da}$ ) with the monosaccharide L-rhamnose (MW $164 \mathrm{Da}$ ). While the exact routes of permeation remain to be clarified, macromolecules such as lactulose are commonly thought to permeate the intestinal mucosa via the size-selective paracellular pathway, while smaller probes such as rhamnose are considered to permeate the mucosa transcellularly as well as paracellularly $(3,15,33)$. Various other sugar probes may be added to the permeability test mix, and by exploiting the differences in digestibility and degradation of these sugar probes, permeability assessment can be performed on the gastroduodenal part, small intestine and large intestine $(26,27)$. Furthermore, quantification of plasma sugar concentrations after intake of the sugar test solution enables detection of moderate and transient changes in GI permeability (35). The use of HPLC-MS allows the quantification of very low sugar concentrations, but this technique is expensive and the system is not yet standard laboratory equipment, thereby limiting the applicability of the sugar- 
based permeability analysis. In addition, sugar permeability analysis may be hampered by the presence of food-derived sugars in urine and plasma $(12,36)$, stressing the importance of an adequate analytical technique for accurate permeability analysis.

Another commonly used option for permeability analysis is based on the application of differently-sized PEG probes. While the addition of PEG to some food products such as artificially sweetened sodas $(8,14)$, and its occasional use in clinical practice for colonic lavage limit its applicability (3), PEG has a number of advantages over the use of ${ }^{51} \mathrm{CrEDTA}$ and sugars for permeability analysis. It does not require radioactivity, is not metabolized by enzymes or degraded by bacteria within the human GI tract $(3,9,10)$, and the required method of analysis is less expensive and time-consuming than for the other probes. Therefore, PEG probes are suggested to be particularly suitable markers for whole gut permeability assessment in man.

The current lack of consensus for GI permeability assessment has led to many studies on intestinal permeability using different probes, which making it very difficult to compare and interpret results. Therefore, our aim was to resolve part of this diversity by comparing urinary probe excretion and accuracy of a PEG-based permeability assay with the DS lactulose-rhamnose test in a double-blinded crossover study, using the non-steroidal anti-inflammatory drug (NSAID) indomethacin to induce a state of increased GI permeability in healthy individuals $(5,6)$.

\section{MATERIALS AND METHODS}

The current study was approved by the medical ethical committee of Maastricht University Medical Center and was conducted according to the revised version of the Declaration of Helsinki (October 2008, Seoul). The study is part of a larger project that has been registered at the US National Library of Medicine (http://www.clinicaltrials.gov, NCT00943345).

\section{Participants}

Nine healthy men and women ( 5 men, 4 women; mean age $31.2 \pm 4.7$ years; body mass index $23.9 \pm 0.9$ ) were included in this study. Subjects were not experiencing abdominal complaints during their normal daily activities, as assessed by a questionnaire prior to inclusion. Exclusion criteria were recent (defined as in the past 30 days) use of any medication, history of abdominal surgery, known hypersensitivity to NSAIDs or food products, pregnancy, alcohol or substance abuse, and any other medical condition that could influence the experimental results (eg gastrointestinal diseases). Volunteers were informed about the nature and risks of the experiments. Written consent was obtained at least 5 days before the experiments. No medication use was permitted during the study period. 


\section{Pre-test restrictions/arrangements}

In the 2 days prior to the experimental test days, test subjects were instructed to maintain normal dietary patterns and to record dietary intake. In addition, subjects were instructed to avoid alcohol, caffeine or spicy products, and to maintain diet as recorded for the subsequent test days to minimize the effects of variations in dietary intake. The evening before each test day, participants received a standardized meal $(1695 \mathrm{~kJ}$, consisting of $62.6 \mathrm{~g}$ carbohydrate, $18.9 \mathrm{~g}$ protein and $7.9 \mathrm{~g}$ of fat). All participants maintained normal activities of daily living and refrained from heavy physical activity in the 2 days prior to each test day. Subjects were kept fasted overnight, but were allowed to drink tap water (maximum of $300 \mathrm{~mL}$ ) in the morning.

\section{Test mix preparation}

The PEG test mix used consisted of 5.7 g PEG 400 (Chempri BV, Raamsdonksveer, the Netherlands), 2.5 g PEG 1500 (Chempri BV) and $13.8 \mathrm{~g}$ of PEG 3350 (Norgine BV, Amsterdam, the Netherlands), dissolved in $150 \mathrm{~mL}$ of tap water, based on previous studies by Parlesak et al and Kerckhoffs et al. (20,29). All PEG used in the test mix were tested for safe oral consumption by Basic Pharma BV (Geleen, the Netherlands). The DS mix consisted of $5 \mathrm{~g}$ lactulose (Centrafarm, Etten-Leur, the Netherlands) and $0.5 \mathrm{~g}$ of L-rhamnose (Danisco Sweeteners, Copenhagen, Denmark), also dissolved in $150 \mathrm{~mL}$ of tap water. Both sugars were intended and tested for safe oral human consumption by the manufacturer.

\section{Study design and sampling}

The current study was randomized controlled and double-blinded in set-up. All subjects completed 4 test days. On all these days, GI permeability analysis was performed, using either PEG probes or DS probes. Subjects were tested twice using the PEG probes; once to assess permeability under basal (placebo) conditions, and once to assess permeability after indomethacin. On two other, separate test days, DS probes were used to assess basal and indomethacin-induced permeability. The interval between test days was at least 7 days, but incidentally extended up to a maximum of 20 days. Subjects received either placebo capsules (to test basal permeability) or capsules with indomethacin (Actavis B.V. Baarn, the Netherlands) $75 \mathrm{mg}$ at 22:00 the evening before the test day and $50 \mathrm{mg}$ at 7:30 (1 hour prior to the start of the tests) to increase GI permeability (6). Capsules were macroscopically identical. Both participants and executing researchers were unaware of the content of the capsules. Participants were instructed to drink $200 \mathrm{~mL}$ of tap water per hour during the test. After ingestion of the probes, urinary samples were collected at baseline and every hour, up to 5 hours after ingestion of the test mix. Urinary volumes were recorded and $4 \mathrm{~mL}$ of each sample was put in a polypropylene tube (Greiner Bio-One, Kremsmünster, Austria) and kept on ice until centrifugation (within 30 minutes after sampling). In addition, subjects collected 5-24 h urine at home and returned the bottles the next morning. Urinary volume was recorded, and urine was immediately processed. 
Baseline blood samples were collected in pre-chilled ethylenediaminetetra-acetic acid (EDTA) tubes (BD vacucontainer, Becton Dickinson Diagnostics, Aalst, Belgium) and immediately centrifuged. Both plasma and urine samples were centrifuged at $4^{\circ} \mathrm{C}$ at $2,300 \mathrm{x}$ for 15 minutes and stored at $-80^{\circ} \mathrm{C}$ until analysis.

\section{Analysis of intestinal epithelial damage and inflammation}

Intestinal epithelial cell damage was assessed by quantification of plasma concentrations of human Intestinal Fatty Acid Binding Protein (I-FABP) using a highly specific in-house developed enzyme linked-immunosorbent assay (ELISA) (detection window 20-5,000 pg/mL), as previously described (35).

Inflammation was determined in the same plasma samples by analysis of calprotectin concentrations using a commercially available calprotectin ELISA (detection window 0.78-50 $\mathrm{ng} / \mathrm{mL}$ ) kindly provided by Hycult Biotechnology (Uden, the Netherlands). Both markers were determined on all experimental days, in each first blood sample of the day $(t=0)$.

\section{Analysis of permeability probes}

All chemicals used for laboratory purposes were of analytical grade, and unless specified otherwise, purchased from Sigma Aldrich (St. Louis, MO). Ultra-pure water was generated though a Super-Q water purification system (Millipore, Billerica, MA) and used as the source of water for all analytical assays.

Urinary sugar concentrations were determined by isocratic ion-exchange chromatography in combination with mass spectrometry detection, as previously described (36).

Urinary PEG concentrations were analyzed by reversed phase HPLC in combination with evaporative light scatter detection (ELSD). The analysis was based on PEG analysis described by Kerckhoffs et al. (20). In short, samples were thawed to room temperature and transferred to $1.5 \mathrm{~mL}$ Eppendorf cups containing $6 \mathrm{mg}$ 5-sulfosalicylic acid. After thorough mixing, samples were centrifuged at 50,000 $\mathrm{x} g$ for 10 minutes at $4^{\circ} \mathrm{C}$ in a high-speed centrifuge (Model Biofuge Stratos, Heraeus, Hanau, Germany). Clear urinary supernatant was injected to the HPLC system (2 Model PU-980 pumps, Jasco Easton, MD) using a WISP auto sampler (Model 715, Waters, Milford, MA). Separation of PEG was achieved by reversed phase chromatography on an Allsphere ODS-2 column (particle size of $3 \mu \mathrm{M}, 150 \times 4.6 \mathrm{~mm}$ ID, Grace Alltech, Deerfield, IL), mounted in a Mistral column oven (Separations Analytical Instruments, Ambacht, the Netherlands) set to $50^{\circ} \mathrm{C}$. The simplified work-up of the urinary samples, using only high-speed centrifugation to prepare the urinary samples, did not lead to accelerated degradation of the Allsphere column. Solvents used were water (solvent A) and methanol/water ( $90 / 10 \mathrm{v} / \mathrm{v}$; solvent B). The gradient of the mobile phase used for separation of the PEG probes was: $0 \mathrm{~min}, 95 \%$ solvent $\mathrm{A} ; 9 \mathrm{~min}, 95 \%$ solvent $\mathrm{A} ; 10 \mathrm{~min}, 70 \%$ solvent $\mathrm{A} ; 11$ min, $45 \%$ solvent A; $12 \mathrm{~min}, 45 \%$ solvent A; $13 \mathrm{~min}, 20 \%$ solvent A; $14 \mathrm{~min}, 10 \%$ solvent $\mathrm{A} ; 15$ min, $10 \%$ solvent $\mathrm{A} ; 16 \mathrm{~min}, 0 \%$ solvent $\mathrm{A}$. PEG detection was performed using ELSD on a model 
2000 ES detector (Grace Alltech). Optimal signal-to-noise ratios for PEG determination were obtained by setting the ELSD detector to a temperature of $50^{\circ} \mathrm{C}$ with a nitrogen gas flow of 1.5 $\mathrm{mL} / \mathrm{min}$ at 5 bar and with the impactor off, while maintaining a gradient flow of $1.00 \mathrm{~mL} / \mathrm{min}$.

PEG concentrations in samples were determined using external standard calibration. Analytical standards were prepared by dissolving analytical grade PEG 400, PEG 1500 and PEG 3350 in water, after which aliquots of this standard were stored at $-80^{\circ} \mathrm{C}$. Standards were run before each set of 10 samples. PEG 400 concentration was measured by injecting $2 \mu \mathrm{l}$ of sample into the detector, and for optimal detection of the lower concentrated PEG 1500 and PEG 3350, the injection volume was increased to a maximum of $100 \mu \mathrm{l}$. If urinary probe concentrations in samples collected at baseline were above zero, these baseline levels were subtracted from concentrations measured in urinary samples collected at subsequent time points. Urinary excretions of sugars and PEG were calculated by multiplying urinary concentrations with urine volume. Urinary recovery of the orally ingested probe dose was calculated and expressed as percentage of the orally ingested dose. Probe ratios were calculated from the urinary excretion values.

Urine spiked with PEG 400 was analyzed before and after addition of indomethacin, and urinary samples containing indomethacin were spiked with PEG 400 to confirm our hypothesis that the presence of indomethacin (MW $358 \mathrm{Da}$ ) did not interfere with PEG 400 analysis in urine.

\section{Statistical analysis}

Statistical analysis was performed using GraphPad Prism (Version 5.00, San Diego, CA). Normality of all data was verified by the Kolmogorov-Smirnov test. Measurements of basal intestinal permeability and permeability after indomethacin intake were compared using the Wilcoxon signed rank test, considering the small number of study participants. For the PEG assay and the DS permeability test, Receiver Operating Characteristics (ROC) curves were plotted to determine the cutoff points to discriminate between basal and indomethacin-induced intestinal permeability in our healthy participants. The ideal cutoff point, defined as the maximum sum of sensitivity and specificity, was reported. Correlations were computed by calculating Spearman correlation coefficients $\left(r_{S}\right)$, unless specifically stated otherwise. Data of 9 healthy individuals with and without intake of indomethacin are presented as mean \pm standard error of the mean (SEM) if most data were normally distributed, or as median (range) if not. $P<0.05$ was considered statistically significant. 


\section{RESULTS}

\section{PEG analysis: linearity, accuracy, and limit of detection}

Linearity of PEG detection was determined by injecting a standard containing the three PEG probes. Calibration curves were linear in a range from 0 to $200 \mu \mathrm{M}$. The results of linear regression analysis of the calibration curves are given in Table 1 . The lower limit of quantification observed at a signal-to-noise ratio of 5:1 was $0.63 \mu \mathrm{M}$ for PEG 400, $0.14 \mu \mathrm{M}$ for PEG 1500, and $0.054 \mu \mathrm{M}$ for PEG 3350. Coefficients of variation were calculated from 10 measurements of urinary samples obtained from different individuals. These samples were spiked with different PEG concentrations ranging from 0 to $100 \mu \mathrm{L}$, resulting in overall coefficients of variation of $3.6 \%$ for PEG 400, $2.7 \%$ for PEG 1500, and $1.2 \%$ for PEG 3350.

Table 1: Slope, intercept and determination coefficient $\left(R^{2}\right)$ of linear regression analysis of the calibration curves of PEG 400, PEG 1500 and PEG 3350

\begin{tabular}{llccc} 
& \multicolumn{1}{c}{ Slope $^{\mathrm{a}}$} & Y-intercept $^{\mathrm{a}}$ & X-intercept & $\mathbf{R}^{2}$ \\
\hline PEG 400 & $8.761 \mathrm{e}+006 \pm 489706$ & $-95362 \pm 43874$ & 0.01088 & 0.9816 \\
PEG 1500 & $7.846 \mathrm{e}+007 \pm 3.468 \mathrm{e}+006$ & $-743532 \pm 310682$ & 0.00948 & 0.9884 \\
PEG 3350 & $1.955 \mathrm{e}+008 \pm 1.388 \mathrm{e}+007$ & $-1.718 \mathrm{e}+006 \pm 816305$ & 0.00879 & 0.9754 \\
\hline
\end{tabular}

${ }^{\text {a }}$ Data are presented as mean $\pm \mathrm{SD}$

\section{Indomethacin induces loss of GI barrier integrity}

To verify the putative loss of GI barrier integrity induced by indomethacin, plasma I-FABP and calprotectin were analyzed. I-FABP levels, determined in plasma samples collected at baseline (ie before intake of permeability probes), were significantly higher after intake of indomethacin (443(206-1306) pg/mL) compared with placebo (336(76-885) pg/mL; $P<0.01$; Figure $1 A$ ), reflecting small intestinal injury in the indomethacin-challenged situation. In line, plasma calprotectin levels increased after intake of indomethacin (116(28-273) $\mathrm{ng} / \mathrm{mL})$, compared with placebo (76(26-138) ng/mL; $P=0.0005$; Figure $1 B$ ). While none of the volunteers experienced GI complaints during the test days or in the week thereafter, these plasma data confirm the putative loss of small intestinal integrity and inflammation in the indomethacin-challenged situation. 


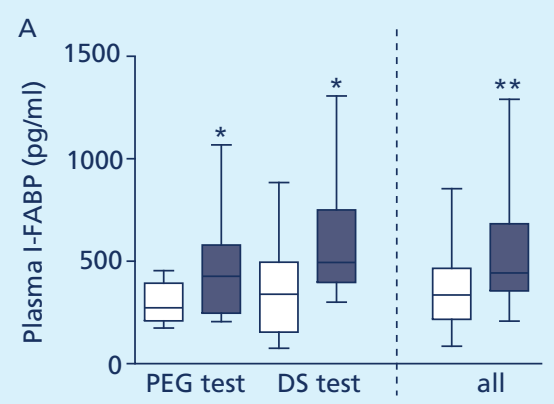

$\square$ Basal
Indomethacin

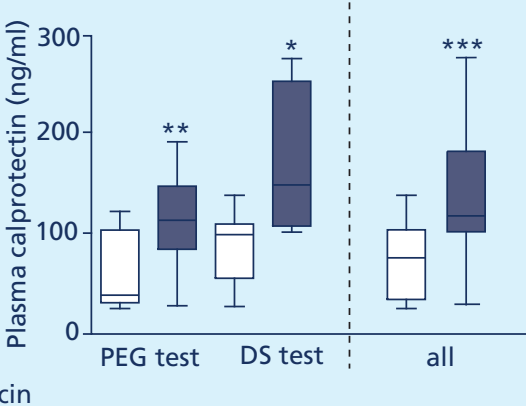

Figure 1: Intake of indomethacin results in small intestinal cell damage and inflammation A) Plasma I-FABP levels increase after intake of indomethacin, indicating indomethacin-induced enterocyte damage. B) Plasma calprotectin levels increase after intake of indomethacin, indicating inflammation after indomethacin ingestion. Data are median (range) of baseline plasma samples $(\mathrm{t}=0)$ of 9 healthy individuals.

Different from basal (placebo) conditions ( ${ }^{\star} P<0.05$; ${ }^{\star \star} P<0.01$; $\left.{ }^{\star \star \star} P=0.0005\right)$.

\section{Indomethacin-induced permeability changes assessed by PEG probes}

\section{Indomethacin increases urinary PEG probe excretion}

Urinary PEG 400 excretion increased significantly after indomethacin-intake compared to basal (placebo) conditions in the $0-1 \mathrm{~h}, 0-2 \mathrm{~h}$, and $0-5 \mathrm{~h}$ urinary collection (Figure 2AB; Table 2), and a trend towards increased 0-24h PEG 400 excretion was observed ( $P=0.06$; Figure $2 A$ ). In line with PEG 400 data, urinary excretion of PEG 1500 increased significantly after intake of indomethacin in the 0-2h and 0-5 urinary collection. No differences in 0-24h PEG 1500 excretion were observed between placebo and the indomethacin-challenged state (Figure 2CD; Table 2). Urinary excretion of the largest probe, PEG 3350, significantly increased after indomethacin intake only in the $0-5$ h urinary collection (Figure 2EF; Table 2).

Interestingly, 2 of the 9 individuals consistently showed decreased 0-2h urinary PEG excretion in the indomethacin-challenged state (Figure 2BDF). Decreased plasma I-FABP levels (454 $\mathrm{pg} / \mathrm{mL}$ at baseline to $433 \mathrm{pg} / \mathrm{mL}$ after indomethacin intake) corroborated the decreased PEG excretion in one of these individuals, suggesting a lack of indomethacin-induced epithelial integrity loss. The other person had increased plasma I-FABP levels but decreased PEG probe excretion after intake of indomethacin. 

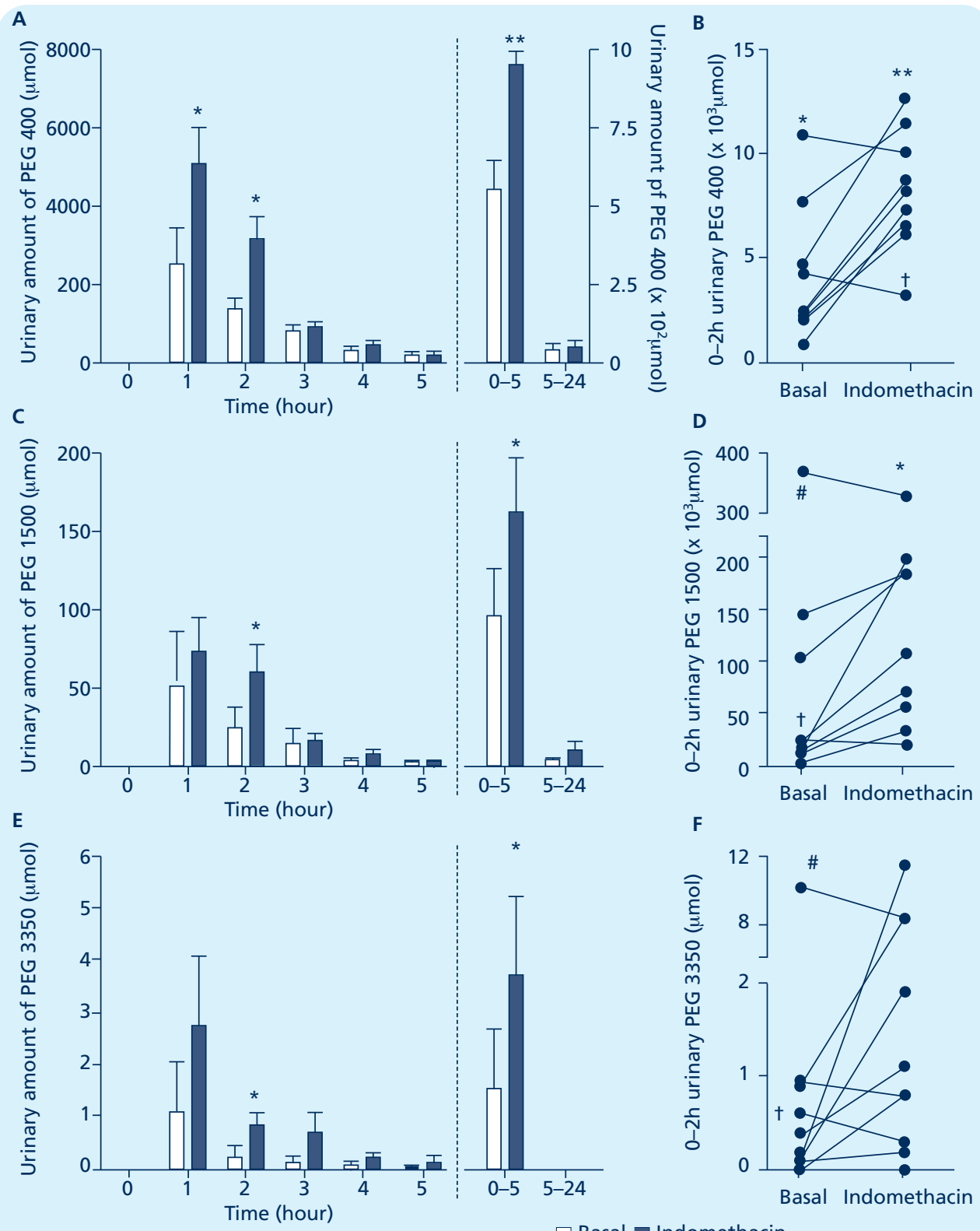

F

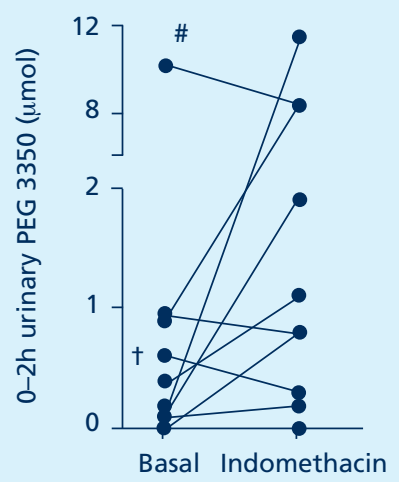

$\square$ Basal $\square$ Indomethacin

Figure 2: Urinary excretion of PEG probes increases after intake of indomethacin

A) Urinary PEG 400 excretion is elevated after intake of indomethacin. B) Individual 0-2h urinary PEG 400 excretion of 9 healthy individuals in basal and indomethacin-challenged state. C) Indomethacin increases the urinary excretion of PEG 1500. D) Individual 0-2h urinary PEG 1500 excretion of 9 healthy individuals in basal and indomethacinchallenged state. E) Urinary excretion of PEG 3350 tends to increase after indomethacin. F) 0-2h urinary PEG 3350 excretion is increased in the majority of the volunteers. Data are mean \pm SEM of 9 healthy individuals unless stated otherwise. Different from basal (placebo) conditions $(\star x<0.05 ; \star \star ~ P<0.01)$. In two volunteers, indicated as $\dagger$ and \# respectively, decreased urinary PEG excretion was observed for each individual PEG probe, while one individual had excessively high indomethacin-induced PEG 3350 excretion in $3 \mathrm{~h}$ and $0-5 \mathrm{~h}$ urine and was therefore not included in figure $2 E$. 
Table 2: Urinary PEG probe excretion in basal (placebo) and indomethacin-challenged state

\begin{tabular}{|c|c|c|c|c|c|}
\hline & \multicolumn{2}{|c|}{$\begin{array}{c}\text { Basal } \\
\text { probe excretion }(\mu \mathrm{mol})\end{array}$} & \multicolumn{2}{|c|}{$\begin{array}{c}\text { Indomethacin } \\
\text { probe excretion }(\mu \mathrm{mol})\end{array}$} & \multirow[t]{2}{*}{$P$} \\
\hline & Median & Range & Median & Range & \\
\hline PEG 400 (0-1h urine) & 1,487 & $(108-8,260)$ & 5,795 & $(1,272-9,923)$ & $<0.05$ \\
\hline PEG $400 \quad(0-2 \mathrm{~h}$ urine $)$ & 1,969 & $(458-11,013)$ & 8,230 & $(2,908-12.942)$ & $<0.01$ \\
\hline PEG 400 (0-5h urine) & 4,597 & $(2,081-12,123)$ & 9,893 & $(4,180-14,808)$ & $<0.01$ \\
\hline PEG 1500 (0-1h urine) & 3.20 & $(0.40-318.30)$ & 35.90 & $(6.70-165.90)$ & 0.20 \\
\hline PEG 1500 (0-2h urine) & 11.90 & $(1.30-383.30)$ & 111.00 & $(22.60-339.60)$ & $<0.05$ \\
\hline PEG 1500 (0-5h urine) & 18.90 & $(2.80-386.50)$ & 174.20 & $(35.50-366.10)$ & 0.05 \\
\hline PEG 3350 (0-1h urine) & 0.00 & $(0.00-8.60)$ & 0.40 & $(0.00-9.80)$ & 0.67 \\
\hline PEG 3350 (0-2h urine) & 0.10 & $(0.00-10.30)$ & 1.10 & $(0.00-11.50)$ & 0.20 \\
\hline PEG 3350 (0-5h urine) & 0.40 & $(0.00-10.30)$ & 1.80 & $(0.00-104.00)$ & $<0.05$ \\
\hline
\end{tabular}

Table 3: Overall accuracy and cutoff points of both urine-based permeability tests

\begin{tabular}{|c|c|c|c|c|c|c|}
\hline & $\begin{array}{l}\text { Urinary } \\
\text { collection }\end{array}$ & $\begin{array}{l}\text { Cutoff } \\
\text { point } \\
(\mu \mathrm{mol})\end{array}$ & Sensitivity & Specificity & $\operatorname{AUC}^{\mathrm{a}}\left(95 \% \mathrm{CI}^{\mathrm{b}}\right)$ & $P$ \\
\hline \multirow[t]{4}{*}{ PEG 400} & $0-1 \mathrm{~h}$ & 2,288 & $89 \%$ & $67 \%$ & $0.79(0.57-1.01)$ & $<0.05$ \\
\hline & $1-2 h$ & 1,507 & $100 \%$ & $67 \%$ & $0.85(0.67-1.03)$ & 0.01 \\
\hline & $0-2 \mathrm{~h}$ & 5,226 & $89 \%$ & $78 \%$ & $0.84(0.65-1.03)$ & $<0.05$ \\
\hline & $0-5 h$ & 7,251 & $89 \%$ & $78 \%$ & $0.83(0.63-1.03)$ & $<0.05$ \\
\hline \multirow[t]{4}{*}{ PEG 1500} & $0-1 \mathrm{~h}$ & 5.00 & $100 \%$ & $56 \%$ & $0.74(0.49-0.99)$ & 0.09 \\
\hline & $1-2 h$ & 9.00 & $100 \%$ & $67 \%$ & $0.79(0.56-1.01)$ & $<0.05$ \\
\hline & $0-2 \mathrm{~h}$ & 20.00 & $100 \%$ & $56 \%$ & $0.77(0.53-1.01)$ & 0.06 \\
\hline & $0-5 \mathrm{~h}$ & 33.00 & $100 \%$ & $67 \%$ & $0.74(0.48-1.00)$ & 0.09 \\
\hline \multirow[t]{4}{*}{ PEG 3350} & $0-1 \mathrm{~h}$ & 0.05 & $78 \%$ & $56 \%$ & $0.64(0.38-0.90)$ & 0.31 \\
\hline & $1-2 \mathrm{~h}$ & 0.15 & $78 \%$ & $78 \%$ & $0.77(0.53-1.00)$ & 0.06 \\
\hline & $0-2 h$ & 0.15 & $89 \%$ & $56 \%$ & $0.74(0.50-0.98)$ & 0.09 \\
\hline & $0-5 h$ & 0.75 & $89 \%$ & $78 \%$ & $0.78(0.54-1.02)$ & $<0.05$ \\
\hline \multirow[t]{4}{*}{ PEG 3350/400 } & $0-1 \mathrm{~h}$ & $5 e-005$ & $67 \%$ & $89 \%$ & $0.77(0.54-1.00)$ & 0.05 \\
\hline & $1-2 h$ & $5 e-005$ & $78 \%$ & $89 \%$ & $0.79(0.55-1.03)$ & $<0.05$ \\
\hline & $0-2 \mathrm{~h}$ & $5 e-005$ & $78 \%$ & $89 \%$ & $0.80(0.58-1.03)$ & $<0.05$ \\
\hline & $0-5 h$ & $5 e-005$ & $89 \%$ & $89 \%$ & $0.85(0.66-1.06)$ & 0.01 \\
\hline \multirow[t]{4}{*}{$\mathrm{L} / \mathrm{R}$ ratio } & $0-1 \mathrm{~h}$ & 0.17 & $75 \%$ & $100 \%$ & $0.85(0.62-1.09)$ & $<0.05$ \\
\hline & $1-2 h$ & 0.105 & $78 \%$ & $89 \%$ & $0.86(0.68-1.04)$ & $<0.05$ \\
\hline & $0-2 \mathrm{~h}$ & 0.13 & $78 \%$ & $100 \%$ & $0.87(0.69-1.07)$ & $<0.05$ \\
\hline & $0-5 \mathrm{~h}$ & 0.18 & $78 \%$ & $78 \%$ & $0.77(0.65-1.08)$ & 0.08 \\
\hline
\end{tabular}



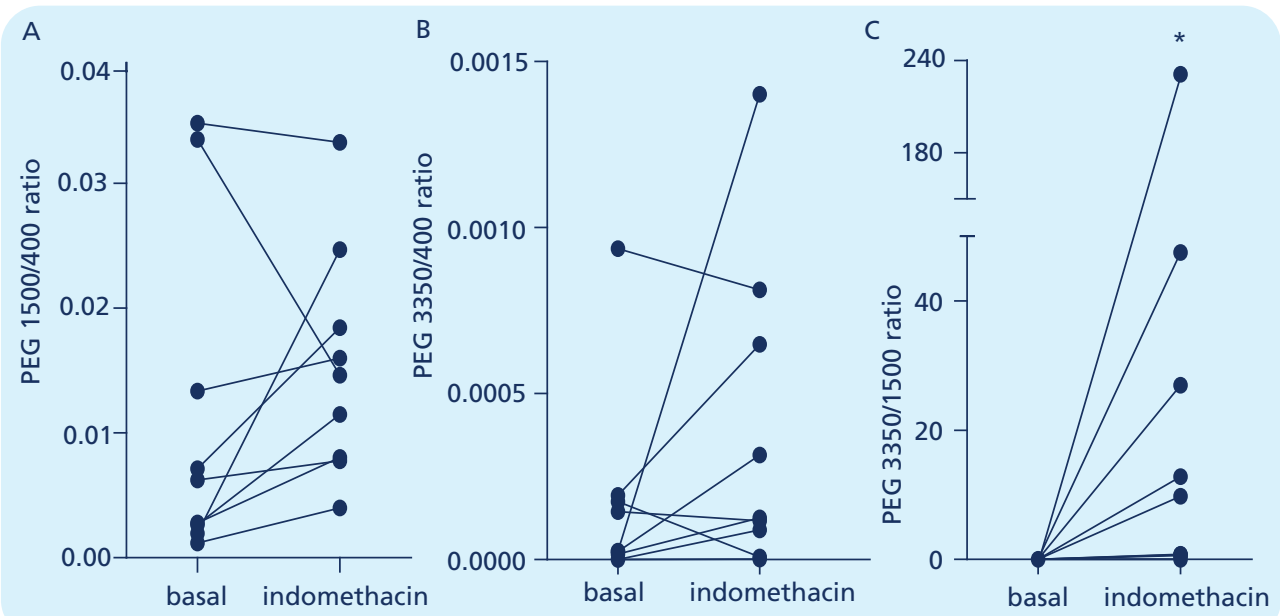

Figure 3: Changes in PEG probe ratios after intake of indomethacin

A) The majority of the volunteers had an increased 0-2h urinary PEG 1500/PEG 400 ratio after indomethacin intake. B) Indomethacin resulted in an increased urinary PEG 3350/PEG 400 ratio in most individuals. C) Individual 0-2h urinary PEG 3350/PEG1500 ratios increased after indomethacin intake $\left({ }^{\star} P<0.05\right)$.

Statistical analysis of the PEG excretion rates revealed that early urinary collections can be used to detect increased permeability with acceptable sensitivity and specificity (Table 3). Alternatively, 0-5h urinary PEG excretion may be used to detect increased GI permeability after indomethacin intake (Table 3).

To increase our understanding of the relation between cellular injury and increased GI permeability, correlations between intestinal cell integrity loss (ie plasma I-FABP levels) and increased GI permeability (i.e. urinary PEG excretion) were studied. Normalized levels of I-FABP, a small 14-15 kDa protein (30) measured in plasma samples collected just before probe intake, correlated significantly with PEG 3350 excretion $\left(R_{S}=0.51 ; P<0.05\right.$ for $0-2 h$ urinary excretion and $R_{S}=0.69 ; P<0.005$ for $0-5$ h urinary excretion), while no correlations were found between I-FABP and other PEG probes. Hence, urinary PEG 3350 excretion correlates with increased I-FABP levels after indomethacin consumption, suggesting a role for enterocyte integrity loss and subsequent release of cytosolic protein I-FABP in the development of intestinal barrier loss reflected by increased PEG 3350 permeation.

\section{Indomethacin and urinary PEG probe ratios}

GI permeability can be assessed either by determination of urinary probe excretion, or by calculation of probe ratios (3). Expressing permeability information as the ratio of a larger probe over a smaller probe has a couple of advantages over the use of single probe urinary excretion rates (3). Most importantly, permeability probe ratios are less affected by individually determined factors unrelated to GI permeability, such as renal clearance (34). Therefore, the three PEG 
A

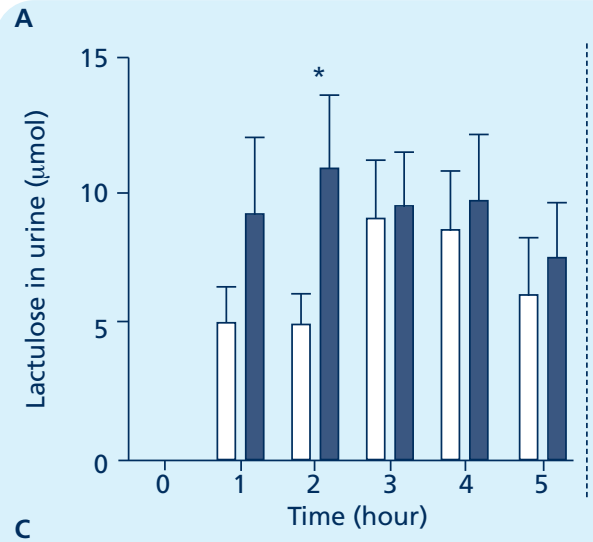

C
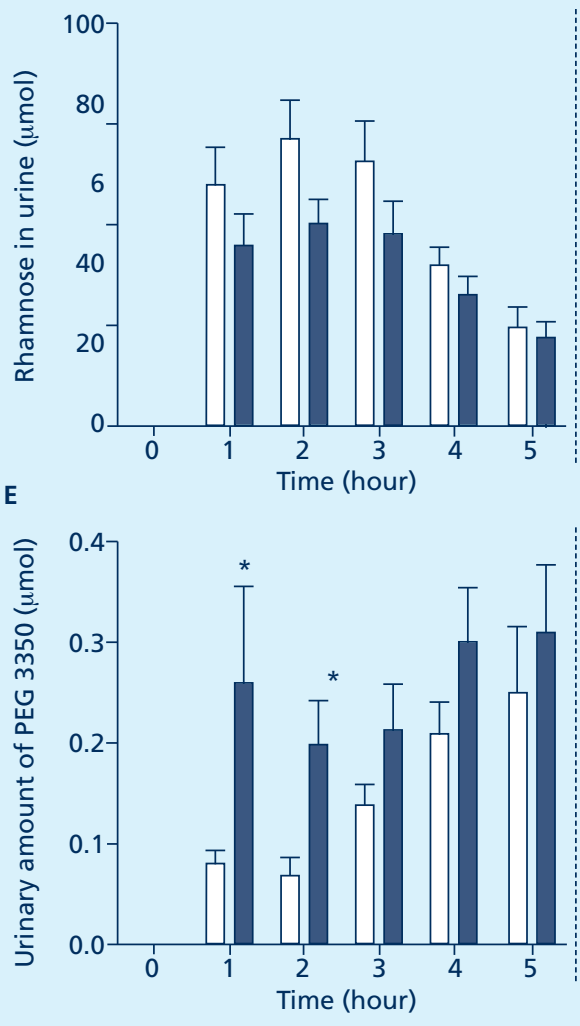
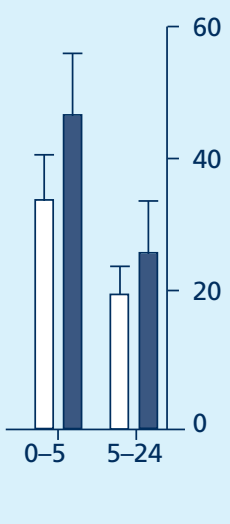

B

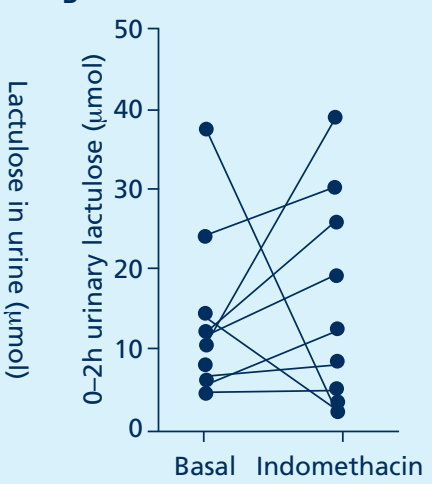

D

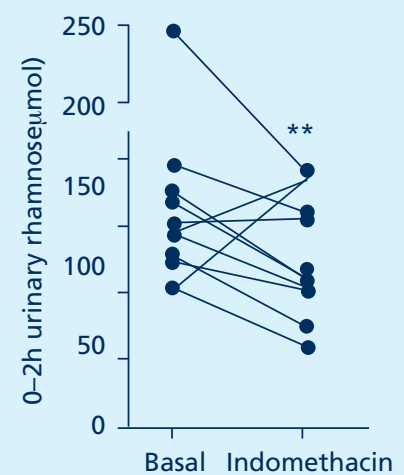

F

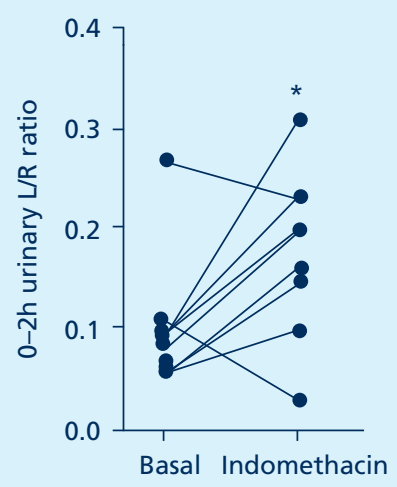

\section{Basal $\square$ Indomethacin}

Figure 4: Urinary excretion of sugar probes increases after intake of indomethacin

A) Urinary excretion of lactulose tended to increase after intake of indomethacin. B) Individual 0-2h urinary lactulose excretion of 9 healthy individuals in basal and indomethacin-challenged state. C) Rhamnose excretion decreased after intake of indomethacin. D) 0-2h urinary rhamnose excretion of 9 healthy individuals decreased significantly in the indomethacin-challenged state. E) Urinary L/R ratio increased after intake of indomethacin, indicating increased intestinal permeability. F) Individual $0-2 \mathrm{~h}$ urinary $\mathrm{L} / \mathrm{R}$ ratios are increased after indomethacin intake. Data are mean \pm SEM of 9 healthy individuals. Different from basal (placebo) conditions $\left({ }^{\star} P<0.05\right.$; ${ }^{\star \star} P<0.01$ ). 
probes were also analyzed as urinary PEG ratios. The PEG ratios determined in the $0-2 \mathrm{~h}$ urinary collections are displayed in Figure 3.

While the majority of individuals showed an increase in all three urinary PEG ratios after indomethacin consumption (Figure 3), no significance was reached. In line with these data, urinary PEG ratios did not correlate with plasma I-FABP levels. Only the PEG 3350/PEG 400 ratio increased significantly after intake of indomethacin compared to basal conditions $(P \leq 0.05$ in $0-1 \mathrm{~h}, 2-3 \mathrm{~h}$, and $0-5 \mathrm{~h}$ urine).

In line with these results, statistical analysis revealed that only the urinary PEG 3350/PEG 400 ratio could accurately detect indomethacin-induced increases in GI permeability (Table 3).

\section{Indomethacin-induced permeability changes assessed by the DS test}

The urinary excretion of the $342 \mathrm{Da}$ sugar probe lactulose increased after intake of indomethacin, an increase that was most pronounced in the first two hours after intake of the sugars (Figure $4 A B$ ). Urinary recovery in these first two hours was roughly $0.110 \pm 0.030 \%$ after intake of indomethacin compared with $0.096 \pm 0.024 \%$ under basal conditions. Two individuals had decreased lactulose excretion in the indomethacin-challenged state, but these individuals were not the same individuals that showed decreased PEG excretion after indomethacin intake.

Urinary excretion of the $164 \mathrm{Da}$ sugar probe rhamnose decreased after intake of indomethacin, reaching significance in the $0-2 \mathrm{~h}$ and $0-5 \mathrm{~h}$ urinary collection $(P<0.01$ and $P<0.05$, respectively; Figure $4 C D$ ).

$0-5 \mathrm{~h}$ urinary rhamnose excretion levels were equivalent to $8.6 \pm 0.8 \%$ and $6.4 \pm 0.5 \%$ urinary recovery after placebo and indomethacin intake, respectively $(P<0.05)$. Due to the increased lactulose excretion and simultaneous decrease in rhamnose excretion, urinary ratios of the large sugar probe lactulose and the smaller rhamnose probe increased significantly after indomethacin intake (Figure 4EF). In keeping with the excretion profiles of lactulose and the PEG probes, this increase was most pronounced in the first two hours after probe intake (Figure 4EF). While lactulose excretion could not provide accurate sensitivity and specificity to detect indomethacininduced intestinal permeability as single probe, urinary $\mathrm{L} / \mathrm{R}$ ratios in these first two hours could accurately detect increased permeability in the indomethacin-challenged state (Table 3). 


\section{DISCUSSION}

Intestinal permeability is an important parameter of gut barrier function, but the availability of multiple permeability tests impedes the interpretation and comparison of urinary probe excretion. The aim of the current study was to compare urinary probe excretion and accuracy of a PEG assay and DS lactulose-rhamnose permeability assay in a double-blinded crossover study, using the NSAID indomethacin to induce increased GI permeability.

Before executing the human study, the time-consuming sample preparation protocol for analysis of the PEG probes was optimized. Analysis of PEG probes in biological samples was previously described by Parlesak et al using HPLC with differential refraction index detection (29). We enhanced the analytical sensitivity of PEG analysis using ELSD detection as described by Kerckhoffs et al (20), while simultaneously reducing the sample preparation protocol to a single sample centrifugation step prior to HPLC analysis. Due to this simple high-speed centrifugation step, the time consuming extraction methods previously required to obtain satisfactory lower limits of detection were no longer necessary $(20,29)$, making PEG analysis quick and easy. Sugar analysis was performed by HPLC-MS detection, as previously described (36). The human study was performed to analyze the application of this optimized PEG approach in a controlled, experimental setting and to compare its results with data obtained using the classical DS test.

Increased GI permeability was previously described after short-term consumption of indomethacin (6). Since indomethacin was used in the current study as a method to increase GI permeability, we first verified the putative loss of GI barrier integrity in our participants. Indeed, significantly increased plasma levels of I-FABP and calprotectin were observed after indomethacin intake, allowing the use of this setting for controlled evaluation of the PEG test and the DS test for GI permeability analysis.

In this experimental setting of integrity loss, urinary excretion of PEG 400, PEG 1500, and PEG 3350 increased after indomethacin consumption. While the absolute excretion rates of the three different-sized PEG probes varied, the urinary excretion profiles of the PEG probes showed high consistency. Urinary excretion of lactulose, the 342 Da sugar probe, increased in the indomethacin-challenged situation in a similar fashion. The indomethacin-induced increases in urinary excretion of PEG and lactulose were most pronounced in the first two hours after probe intake. The latter suggests the presence of increased upper GI permeability after indomethacin intake, which correlates with the established injury profile of NSAIDs, especially affecting the upper GI tract and small intestine (17).

It is thought that urinary permeability ratios of two different-sized probes provide more reliable information on GI permeability than single probe excretion rates in case of a one-time analysis (3). The main reason for this assumption is that urinary probe ratios are less affected by every-day variations in urinary output due to gastric emptying, intestinal transit, et cetera (3). In the current study, both the PEG probes and the sugar probes were expressed as urinary ratios 
and as single probe excretion rates. PEG probes are not commonly expressed as excretion ratios $(21,22)$, and since the excretion of all three PEG probes increased after indomethacin consumption, the increase in urinary PEG ratios was not very pronounced in this study. Only the PEG 3350/PEG 400 ratio could detect increased GI permeability after indomethacin use with accurate sensitivity and specificity. Compared to the PEG ratios, the urinary L/R ratio increased more distinctly, especially in the first two hours after probe intake, due to an indomethacininduced increase in lactulose excretion and a simultaneous decrease in urinary rhamnose excretion. The standing theory is that large ( $>300 \mathrm{Da}$ ) probes permeate the GI mucosa paracellularly, while smaller probes such as rhamnose travel via both paracellular and transcellular routes (28). This theory dictates that the PEG-based permeability assay provides information on the paracellular permeability pathway (23), while the dual sugar test assesses both the paracellular and transcellular pathway. However, since the diffusion of water also occurs transcellularly, a hyperosmolar luminal content such as the 5-g lactulose dose given in the current study may affect the permeability of the small, transcellular traveling probes by directing the water and small probe (i.e. rhamnose) flux towards the hyperosmolar intestinal lumen (2). It remains to be clarified whether changes in osmolarity affect the reliability of the permeability test.

Our aim was to compare urinary probe excretion as well as the accuracy of both tests. The main finding of this study was that the urinary excretion of the large ( $>300 \mathrm{Da}$ ) probes, the urinary probe ratios, and the accuracy of the tests are very much alike. Single PEG 400 excretion, the PEG 3350/PEG 400 ratio and the L/R ratio determined in 0-2h urine after probe intake all provided accurate detection of indomethacin-induced increases in GI permeability. The L/R cutoff point of 0.18 in $0-5 \mathrm{~h}$ urine is in line with data from previous studies using the DS test after short-term indomethacin consumption $(7,32)$ and prolonged indomethacin intake $(24,25,31)$. Unfortunately, no cutoff points were reported in previous studies using PEG for permeability analysis in healthy individuals after indomethacin. Previously, Bjarnason et al. studied the intestinal permeability of healthy subjects and patients with celiac disease with PEG 400, lactulose, rhamnose, and ${ }^{51} \mathrm{CrEDTA}$, and found similar excretion and sensitivity rates for both the DS test and ${ }^{51}$ CrEDTA permeability test (4). However, PEG 400 excretion was decreased in the latter study in case of increased permeability due to celiac disease (4). We have to conclude that due to the numerous factors that influence urinary probe excretion, cutoff points and accuracy data, one needs to evaluate the different permeability tests in one study in order to enable a correct comparison of test performance.

Data of the current study improve our knowledge of permeability probe excretion by giving a clear overview of urinary PEG and sugar probe excretion over time in a controlled, experimental setting. Future studies are warranted to evaluate the applicability of the permeability tests in clinical settings, e.g. for permeability assessment in patients with intestinal diseases such as celiac disease. Based on the current findings, we consider that both tests may be used to assess GI permeability, using urinary probe ratios if multiple testing is impossible such as in patients 
with active disease. Furthermore, we recommend combining GI permeability assessment with the analysis of plasma I-FABP and calprotectin levels, providing a more thorough overview of GI mucosal integrity. PEG analysis may provide information on whole-gut permeability, while the dual sugar assay requires additional, inert sugars in the test mix to allow large intestinal permeability analysis (3).

In conclusion, the current study demonstrates that GI permeability tests based on the urinary excretion of PEG and lactulose-rhamnose show equivalent performance in healthy individuals after NSAID consumption. Since a clear demand for a standardized whole gut permeability test continues to exist, efforts to develop the ideal permeability test should be continued. 


\section{REFERENCES}

1. Besselink MG, van Santvoort HC, Renooij W, de Smet MB, Boermeester MA, Fischer K, Timmerman HM, Ahmed Ali U, Cirkel GA, Bollen TL, van Ramshorst B, Schaapherder AF, Witteman BJ, Ploeg RJ, van Goor H, van Laarhoven CJ, Tan AC, Brink MA, van der Harst E, Wahab PJ, van Eijck CH, Dejong CH, van Erpecum KJ, Akkermans LM, and Gooszen HG. Intestinal barrier dysfunction in a randomized trial of a specific probiotic composition in acute pancreatitis. Annals of surgery 250: 712-719, 2009.

2. Bijlsma PB, Fihn BM, Sjoqvist A, Groot JA, Taminiau JA, and Jodal M. Water absorption enhances the uptake of mannitol and decreases Cr-EDTA/mannitol permeability ratios in cat small intestine in situ. Scand $\mathcal{F}$ Gastroenterol 37: 799-806, 2002.

3. Bjarnason I, MacPherson A, and Hollander D. Intestinal permeability: an overview. Gastroenterology 108: 15661581, 1995.

4. Bjarnason I, Maxton D, Reynolds AP, Catt S, Peters TJ, and Menzies IS. Comparison of four markers of intestinal permeability in control subjects and patients with coeliac disease. Scand F Gastroenterol 29: 630-639, 1994.

5. Bjarnason I, and Takeuchi K. Intestinal permeability in the pathogenesis of NSAID-induced enteropathy. $\mathcal{F}$ Gastroenterol 44 Suppl 19: 23-29, 2009.

6. Bjarnason I, Williams P, Smethurst P, Peters TJ, and Levi AJ. Effect of non-steroidal anti-inflammatory drugs and prostaglandins on the permeability of the human small intestine. Gut 27: 1292-1297, 1986.

7. Bours MJ, Bos HJ, Meddings JB, Brummer RJ, van den Brandt PA, and Dagnelie PC. Effects of oral adenosine 5 '-triphosphate and adenosine in enteric-coated capsules on indomethacin-induced permeability changes in the human small intestine: a randomized cross-over study. BMC Gastroenterol 7: 23, 2007.

8. Castle L, Cloke HR, Crews C, and Gilbert J. The migration of propylene glycol, mono-, di-, and triethylene glycols from regenerated cellulose film into food. Z Lebensm Unters Forsch 187: 463-467, 1988.

9. Chadwick VS, Phillips SF, and Hofmann AF. Measurements of intestinal permeability using low molecular weight polyethylene glycols (PEG 400). I. Chemical analysis and biological properties of PEG 400. Gastroenterology 73: 241-246, 1977.

10. Chadwick VS, Phillips SF, and Hofmann AF. Measurements of intestinal permeability using low molecular weight polyethylene glycols (PEG 400). II. Application to normal and abnormal permeability states in man and animals. Gastroenterology 73: 247-251, 1977.

11. Chang M, Kistler EB, and Schmid-Schonbein GW. Disruption of the mucosal barrier during gut ischemia allows entry of digestive enzymes into the intestinal wall. Shock 37: 297-305, 2012.

12. Cox MA, Lewis KO, and Cooper BT. Measurement of small intestinal permeability markers, lactulose, and mannitol in serum: results in celiac disease. Digestive diseases and sciences 44: 402-406, 1999.

13. Davies NM. Review article: non-steroidal anti-inflammatory drug-induced gastrointestinal permeability. Alimentary pharmacology \& therapeutics 12: 303-320, 1998.

14. EFSA. Opinion of the Scientific Panel on Food Additives, Flavourings, Processing Aids and Materials in Contact with Food on a request from the Commission related to an application on the use of polyethylene glycol (PEG) as a film coating agent for use in food supplement products. The EFSA fournal 414: 1-22, 2006.

15. Fihn BM, Sjoqvist A, and Jodal M. Permeability of the rat small intestinal epithelium along the villus-crypt axis: effects of glucose transport. Gastroenterology 119: 1029-1036, 2000.

16. Fink MP DR. Epithelial barrier dysfunction: a unifying theme to explain the pathogenesis of multiple organ dysfunction at the cellular level. Critical Care Clinic 21: 177-196, 2005.

17. Garcia Rodriguez LA, and Jick H. Risk of upper gastrointestinal bleeding and perforation associated with individual non-steroidal anti-inflammatory drugs. Lancet 343: 769-772, 1994.

18. Heyman M, Abed J, Lebreton C, and Cerf-Bensussan N. Intestinal permeability in coeliac disease: insight into mechanisms and relevance to pathogenesis. Gut 2011.

19. Katz KD, Hollander D, Vadheim CM, McElree C, Delahunty T, Dadufalza VD, Krugliak P, and Rotter JI. Intestinal permeability in patients with Crohn's disease and their healthy relatives. Gastroenterology 97: 927-931, 1989.

20. Kerckhoffs AP, Akkermans LM, de Smet MB, Besselink MG, Hietbrink F, Bartelink IH, Busschers WB, Samsom $\mathrm{M}$, and Renooij W. Intestinal permeability in irritable bowel syndrome patients: effects of NSAIDs. Digestive diseases and sciences 55: 716-723, 2010.

21. Lee S, Son SC, Han MJ, Kim WJ, Kim SH, Kim HR, Jeon WK, Park KH, and Shin MG. Increased intestinal macromolecular permeability and urine nitrite excretion associated with liver cirrhosis with ascites. World $\mathcal{F}$ Gastroenterol 14: 3884-3890, 2008.

22. Loret S, Nollevaux G, Remacle R, Klimek M, Barakat I, Deloyer P, Grandfils C, and Dandrifosse G. Analysis of PEG 400 and 4000 in urine for gut permeability assessment using solid phase extraction and gel permeation chromatography with refractometric detection. f Chromatogr B Analyt Technol Biomed Life Sci 805: 195-202, 2004. 
23. Ma TY, Hollander D, Krugliak P, and Katz K. PEG 400, a hydrophilic molecular probe for measuring intestinal permeability. Gastroenterology 98: 39-46, 1990.

24. Mahmood A, FitzGerald AJ, Marchbank T, Ntatsaki E, Murray D, Ghosh S, and Playford RJ. Zinc carnosine, a health food supplement that stabilises small bowel integrity and stimulates gut repair processes. Gut 56: 168$175,2007$.

25. Marchbank T, Limdi JK, Mahmood A, Elia G, and Playford RJ. Clinical trial: protective effect of a commercial fish protein hydrolysate against indomethacin (NSAID)-induced small intestinal injury. Alimentary pharmacology E therapeutics 28: 799-804, 2008.

26. Meddings JB, and Gibbons I. Discrimination of site-specific alterations in gastrointestinal permeability in the rat. Gastroenterology 114: 83-92, 1998.

27. Meddings JB, Sutherland LR, Byles NI, and Wallace JL. Sucrose: a novel permeability marker for gastroduodenal disease. Gastroenterology 104: 1619-1626, 1993.

28. Menzies IS, Zuckerman MJ, Nukajam WS, Somasundaram SG, Murphy B, Jenkins AP, Crane RS, and Gregory GG. Geography of intestinal permeability and absorption. Gut 44: 483-489, 1999.

29. Parlesak A, Bode JC, and Bode C. Parallel determination of gut permeability in man with M(r) 400, M(r) 1500, M(r) 4000 and M(r) 10,000 polyethylene glycol. Eur f Clin Chem Clin Biochem 32: 813-820, 1994.

30. Pelsers MM, Hermens WT, and Glatz JF. Fatty acid-binding proteins as plasma markers of tissue injury. Clinica chimica acta; international journal of clinical chemistry 352: 15-35, 2005.

31. Playford RJ, MacDonald CE, Calnan DP, Floyd DN, Podas T, Johnson W, Wicks AC, Bashir O, and Marchbank T. Co-administration of the health food supplement, bovine colostrum, reduces the acute non-steroidal antiinflammatory drug-induced increase in intestinal permeability. Clin Sci (Lond) 100: 627-633, 2001.

32. Troost FJ, Saris WH, and Brummer RJ. Recombinant human lactoferrin ingestion attenuates indomethacininduced enteropathy in vivo in healthy volunteers. Eur f Clin Nutr 57: 1579-1585, 2003.

33. Van Itallie CM, Holmes J, Bridges A, Gookin JL, Coccaro MR, Proctor W, Colegio OR, and Anderson JM. The density of small tight junction pores varies among cell types and is increased by expression of claudin-2. J Cell Sci 121: 298-305, 2008.

34. van Nieuwenhoven MA, de Swart EA, van Eijk HM, Deutz NE, Brouns F, and Brummer RJ. Effects of preand post-absorptive factors on the lactulose/rhamnose gut permeability test. Clin Sci (Lond) 98: 349-353, 2000.

35. van Wijck K, Lenaerts K, van Loon LJ, Peters WH, Buurman WA, and Dejong CH. Exercise-induced splanchnic hypoperfusion results in gut dysfunction in healthy men. PloS ONE 6: e22366, 2011.

36. van Wijck K, van Eijk HM, Buurman WA, Dejong CH, and Lenaerts K. Novel analytical approach to a multisugar whole gut permeability assay. F Chromatogr B Analyt Technol Biomed Life Sci 879: 2794-2801, 2011.

37.Zeng W. Communicating radiation exposure: a simple approach. F Nucl Med Technol 29: 156-158, 2001.

38. Zuckerman MJ, Watts MT, Bhatt BD, and Ho H. Intestinal permeability to [51 Cr]EDTA in infectious diarrhea. Digestive diseases and sciences 38: 1651-1657, 1993. 


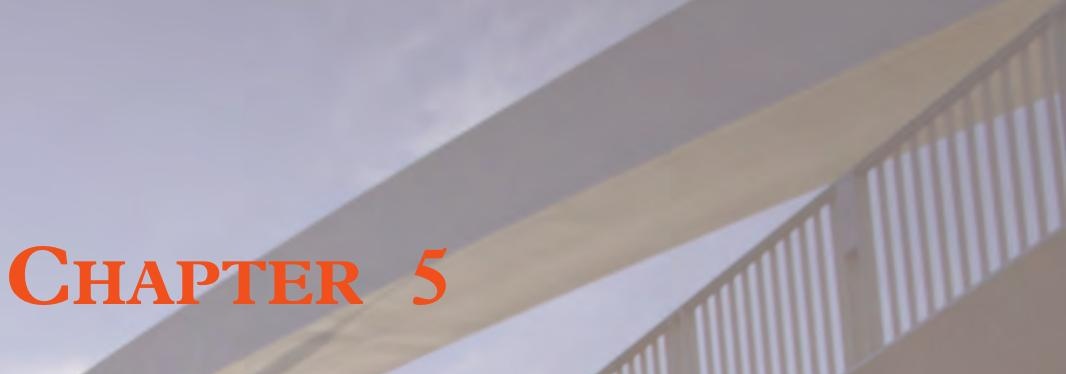

Novel analytical approach to a multi-sugar whole gut permeability assay

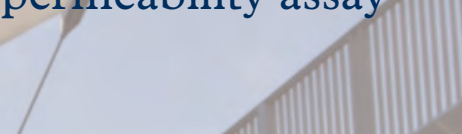

F Chromatogr B Analyt Technol Biomed Life Sci

Volume 879, Issue 26, 2794-2801, 2011

Kim van Wijck ${ }^{1}$

Hans $M H$ van Eijk ${ }^{1}$

Wim A Buurman

Cornelis HC Dejong

Kaatje Lenaerts
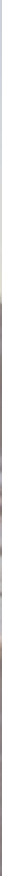

क्ये 


\section{ABSTRACT}

Many pathophysiological conditions are associated with increased gastrointestinal permeability, reflecting an elevated risk of endotoxaemia, inflammation, and sepsis. Permeability tests are increasingly used in clinical practice to obtain information on gastrointestinal functioning, but tests are often restricted to the small intestine, and require large oral sugar doses. Therefore, a novel multi-sugar assay was developed, allowing assessment of whole gut permeability changes in urinary and plasma samples collected at regular intervals from 10 healthy volunteers at baseline and after intake of monosaccharides (rhamnose and erythritol) and disaccharides (sucrose, lactulose, and sucralose). Samples were analyzed by isocratic cation-exchange LC-MS. Sample preparation and detection conditions were optimized. After centrifugation, chromatographic separation was achieved on an IOA- 1000 column set at $30^{\circ} \mathrm{C}$. Column effluent was mixed with ammonia for sugar adduct formation. The lower limit of detection was $0.05 \mu \mathrm{mol} / \mathrm{L}$ for disaccharides and $0.1 \mu \mathrm{mol} / \mathrm{L}$ for monosaccharides. Linearity for each probe was between 1 to 1,000 $\mu \mathrm{mol} / \mathrm{L}$ (R2 0.9987-0.9999). Coefficients of variation were $<5 \%$ in urine, and $<9 \%$ in plasma. Recovery data were within the $90 \%$ to $110 \%$ range at all spiked concentrations. This highly sensitive novel LC-MS approach resulted in a significant decrease of the detection limit for all sugar probes, allowing a 5-fold reduction of the commonly used lactulose dose and the addition of sugar probes to assess also gastroduodenal and colon permeability. In combination with its extended application in plasma, these features make the novel assay a promising tool in the assessment of site-specific changes in gastrointestinal permeability in clinical practice. 


\section{INTRODUCTION}

Many pathophysiological conditions are associated with increased gastrointestinal (GI) permeability, including celiac disease (8), inflammatory bowel disease (10), and acute pancreatitis (3). Increased intestinal permeability has even been reported in patients with chronic heart failure (19) and in elite long distance runners (13), probably as a result of intestinal hypoperfusion. Increased GI permeability is an undesirable condition that renders the host more susceptible to noxious compounds present in the gut lumen, including microbes and their products (17), potentially leading to systemic endotoxaemia and sepsis. Permeability analysis is considered to provide crucial information on GI functioning, and it is increasingly used in clinical practice and research (4). Classically, human GI permeability is assessed by measurement of the urinary excretion of an orally administered disaccharide (usually lactulose) and a monosaccharide (mannitol or L-rhamnose). The urinary excretion ratio of the two sugars is considered to be a parameter of small intestinal permeability. A common technique for determining these urinary sugars concentrations is HPLC in combination with refractive index detection (12). However, there is room for significant analytical improvement (4). Therefore, the aim of this study was to improve the classical sugar-based permeability test on three different levels.

First, the intake of the relatively large amount of lactulose (5 g) used in the classical sugar test can influence GI function, probably by increasing the speed of passage through the GI system, thereby affecting the sensitivity of the permeability analysis $(5,18,25)$. The lack of sensitivity and specificity of the existing methodology currently prevents the use of lower sugar doses. Increasing the analytical sensitivity was therefore our key target in this study. Secondly, the colonic degradation of the classical sugar probes restricts the usefulness of the dual sugar assay to the small intestine. In addition, the classical dual sugar test does not allow specific analysis of the upper part of the duodenum. The ideal assay includes additional probes to provide specific permeability information on the upper GI tract, i.e. the stomach and duodenal bulb, and the colon. Such an expanded sugar test mix would theoretically affect intestinal transit, if applied at the classical dose. Hence, expansion of the sugar test composition requires a further increase in analytical sensitivity to reduce the cumulative oral sugar dose, and requires a specific method of detection to assess all applied sugar probes in a single analytical run. Thirdly, the dual sugar assay is classically performed in $0-5 \mathrm{~h}$ urinary collections, and plasma analysis is not commonly performed. Nevertheless, frequent plasma sampling provides advantages over urinary permeability analysis, since it enables the detection of modest, transient gastrointestinal permeability changes. Plasma analysis requires a more extensive sample preparation, and a more sensitive analysis, since plasma sugar concentrations are low compared to urinary concentrations. Therefore, a novel, more sensitive approach was set up, enabling the analysis of multiple sugar probes in urine and plasma. Here, we describe this analytical method which is based on liquid chromatography in combination with mass spectrometry (LC-MS), and the potential advantages of the novel multi-sugar permeability assay for application in clinical practice. 


\section{MATERIALS AND METHODS}

The study was approved by the medical ethical committee of the Maastricht University Medical Center and conducted according to the revised version of the Declaration of Helsinki (October 2008, Seoul).

\section{Materials}

All chemicals used for laboratory purposes were of analytical grade, and unless specified otherwise, purchased from Sigma Aldrich (St. Louis, MO). Ultra-pure water was generated through a Super-Q water purification system (Millipore, Billerica, MA) and used as the source of water throughout the assay.

The permeability test mix consisted of $1 \mathrm{~g}$ sucrose (342.3 Da; Van Gilse, Suiker Unie, Dinteloord, the Netherlands), 1 g lactulose (342.3 Da; Centrafarm B.V., Etten-Leur, the Netherlands), $1 \mathrm{~g}$ sucralose (397.6 Da; Brenntag Mülheim an der Ruhr, Germany), $1 \mathrm{~g}$ erythritol (122.1 Da; Danisco Sweeteners, Copenhagen, Denmark) and $0.5 \mathrm{~g}$ of L-rhamnose (rhamnose; 164.2 Da; Danisco Sweeteners, Copenhagen, Denmark), freshly dissolved in $150 \mathrm{~mL}$ of tap water. All products used in the test mix were intended and tested for safe oral consumption. The purity of these sugar probes was reported to be $98.0-99.9 \%$.

\section{Preparation of standards}

Standards were prepared by dissolving analytical grade sugars into Super-Q water to a concentration of $10 \mathrm{mmol} / \mathrm{L}$ each. Next, a mixture was composed of each individual sugar to a final concentration of $500 \mu \mathrm{mol} / \mathrm{L}$ and aliquots were stored at $-80^{\circ} \mathrm{C}$.

\section{Sampling from healthy human volunteers}

Included were 10 healthy men and women aged 18-75 years. The volunteers did not take medication in the month prior to participation, and had no history of GI disease or abdominal surgery. The volunteers were informed about the nature and risks of the experiments. Written consent was obtained 5 days before the experiments. Subjects refrained from strenuous physical activity and from oral intake of caffeine, alcohol, spicy foods and drinks, and any of the sugars used in the permeability test from 2 days before, to the morning after the test day.

Subjects were tested after an overnight fast. In short, a catheter (20 Gauge, B. Braun, Melsungen, Germany) was placed in the participant's forearm vein. Blood was collected into prechilled ethylene-diamine-tetra-acetic acid tubes (Vacucontainer, Becton Dickinson, Franklin Lakes, NJ), and kept on ice. Urine was collected by the subjects in plastic cups of $1 \mathrm{~L}$, after which urinary volume was recorded and $4 \mathrm{~mL}$ was directly transferred to pre-chilled polypropylene tubes (Greiner Bio-One, Kremsmünster, Austria). Blood and urine samples were centrifuged within one hour at $4^{\circ} \mathrm{C}$ at $2300 \times \mathrm{g}$ for 15 minutes and aliquoted. Both plasma and 
urine were immediately stored at $-80^{\circ} \mathrm{C}$ until analysis. Following the collection of baseline plasma and urine samples, the test subjects ingested the test mix described above. Plasma samples were collected every $20 \mathrm{~min}$ and urine every hour until $5 \mathrm{~h}$ after intake of the test mix. Moreover, all urine produced in the 5-24 h interval after intake was collected by the subjects in a $3 \mathrm{~L}$ flask, which was returned to the laboratory the following morning and immediately processed as above.

\section{Sample preparation}

Due to the selectivity of the LC-MS approach, we could suffice with centrifugation of the urinary samples. Aliquots with $500 \mu \mathrm{L}$ of urine were thawed to room temperature, transferred to 1.5 $\mathrm{mL}$ Eppendorf cups, centrifuged at $50,000 \mathrm{xg}$ for $10 \mathrm{~min}$ at $4{ }^{\circ} \mathrm{C}$ in a high-speed centrifuge (model Biofuge Stratos, Heraeus, Hanau, Germany). Clear urine supernatant (200 $\mu \mathrm{L}$ ) was transferred into a $300 \mu \mathrm{L}$ glass insert; spring loaded in a $4 \mathrm{mLWISP}$ style vial (Waters, Milford, MA) and placed into a Peltier chilled Gilson 233 XL sample processor (Gilson, Middleton, WI).

Two plasma sample preparation protocols were tested to remove plasma proteins. The first one involved the addition of sulfosalicylic acid (SSA) after which the samples were vortexed and centrifuged. In the second protocol, $300 \mu \mathrm{L}$ of sample was transferred to Eppendorf cups containing a 3,000 Da cut-off filter (Amicon Ultra $0.5 \mathrm{ml} 3 \mathrm{~K}$, Millipore) to remove the plasma proteins. The filter cups were centrifuged for $30 \mathrm{~min}$ at $11,000 \mathrm{xg}$ at $4^{\circ} \mathrm{C}$, and clear plasma filtrate $(200 \mu \mathrm{L})$ was inserted in the Gilson sample processor as described.

\section{Optimized LC-MS procedure}

Chromatographic separation was based on isocratic elution of individual sugars probes on an IOA-1000 $9 \mu \mathrm{m}$ cation exchange column (300 mm x 7.8 mm ID; Grace, Deerfield, IL), mounted in a Mistral column oven (Separations, H.I. Ambacht, the Netherlands) at $30^{\circ} \mathrm{C}$. An aqueous solution of $20 \mathrm{mmol} / \mathrm{L}$ formic acid and $10 \mathrm{mmol} / \mathrm{L}$ trichloroacetic acid (TCA), was delivered using a Model PU-1580 HPLC pump (Jasco Easton, MD) at a flow rate of $0.225 \mathrm{~mL} / \mathrm{min}$. Samples and standards were injected using a Model 233XL sample processor with Peltier chilled sample storage compartments $\left(10^{\circ} \mathrm{C}\right)$, equipped with a $20 \mu \mathrm{L}$ sample loop (Gilson, Middleton, WI). After separation, the column effluent was mixed with $30 \mathrm{mmol} / \mathrm{L}$ ammonia in $20 \%$ methanol/water (v/v) delivered by an additional Model PU 980 pump (pump B, Figure 1) at a flow of $0.15 \mathrm{~mL} / \mathrm{min}$ to allow the formation of ammonium adducts. MS detection was performed using a model LTQ XL (Thermo Fisher Scientific, Waltham, MA) equipped with an ion-Max electrospray probe. The MS was operated in positive mode. Spray voltage was $4.8 \mathrm{kV}$. Sheath and auxiliary gas were 99 and 30 units, respectively, while capillary temperature was $220^{\circ} \mathrm{C}$. The system was set to a mass range of $125-460 \mathrm{Da}$ in full scan enhanced mode. 


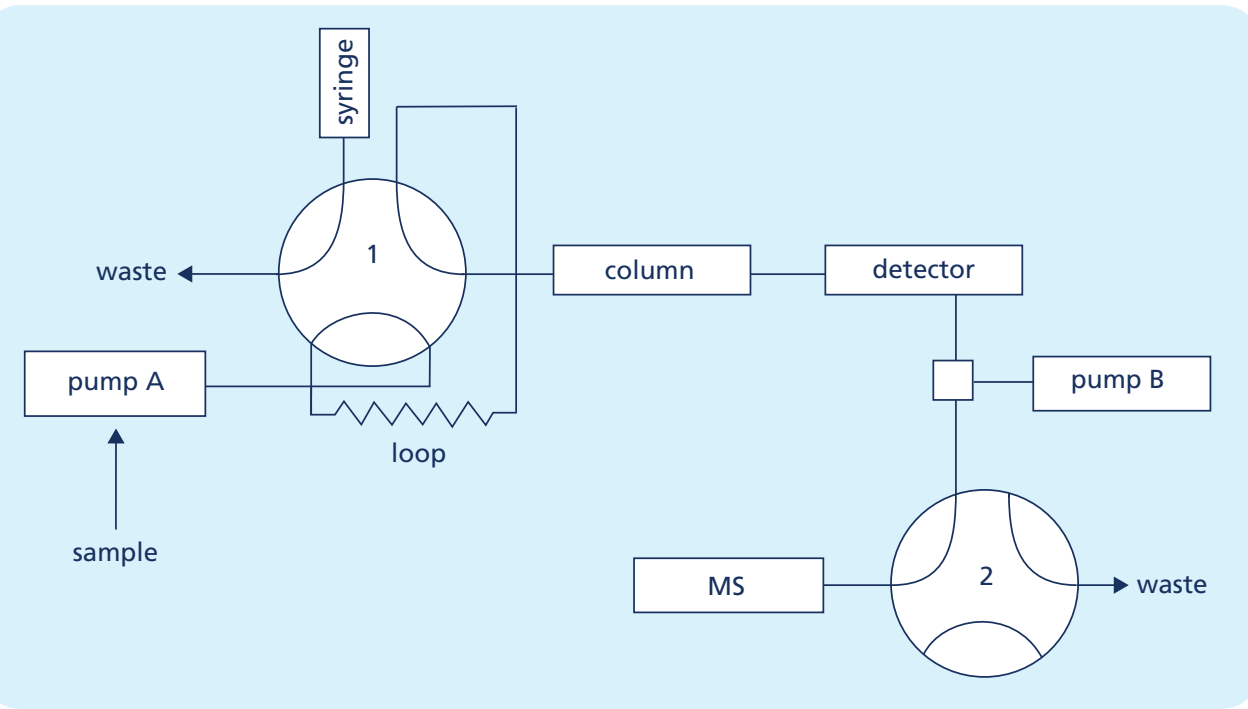

Figure 1: Schematic set-up of the detection system

Samples are delivered by pump A to the solvent stream via valve 1 , which is equipped with a $20 \mu \mathrm{L}$ sample loop. The solvent is then directed to the cation exchange column, mounted in a thermostatic oven. After separation by the column, the solvent goes through the detector, and is mixed with an ammonia/methanol mixture delivered by pump B to allow formation of sugar-ammonium adducts. Finally, the solvent stream is directed to the MS system through valve 2 .

\section{RESULTS}

\section{Optimizing of chromatographic procedure}

To obtain optimal chromatographic results, two approaches were tested. The first involved the use of straight phase chromatography using a carbohydrate ES column (Grace) as described before (20). As we intended to apply MS for detection, ionization of sugars was a prerequisite. Adduct formation using sodium (11), iodine (16), formic acid (1), and ammonia (26) has been described. In our setting, sodium rapidly blocked the ionization probe, while iodine contaminated the ion trap considerably. Adducts formed by formic acid or ammonia showed better results, with the latter giving a more abundant response. However, formic acid and ammonium adducts are only generated at very low concentrations of organic solvent (acetonitrile), while high acetonitrile concentrations are required for straight phase gradient elution of chromatographic separation. One way to solve this inconsistency is to apply isocratic elution of the sugars, and exploit the differences in molecular weight to identify and quantify the sugars (26). Unfortunately, physiological samples contain many endogenous isobaric sugars which have to be separated from the exogenously applied sugar probes for their correct quantification. Consequently, we studied the application of cation-exchange chromatography to separate the sugar probes of interest from the endogenous isobaric sugars. From all columns tested, only the IOA-1000 $9 \mu \mathrm{m}$ column (300 

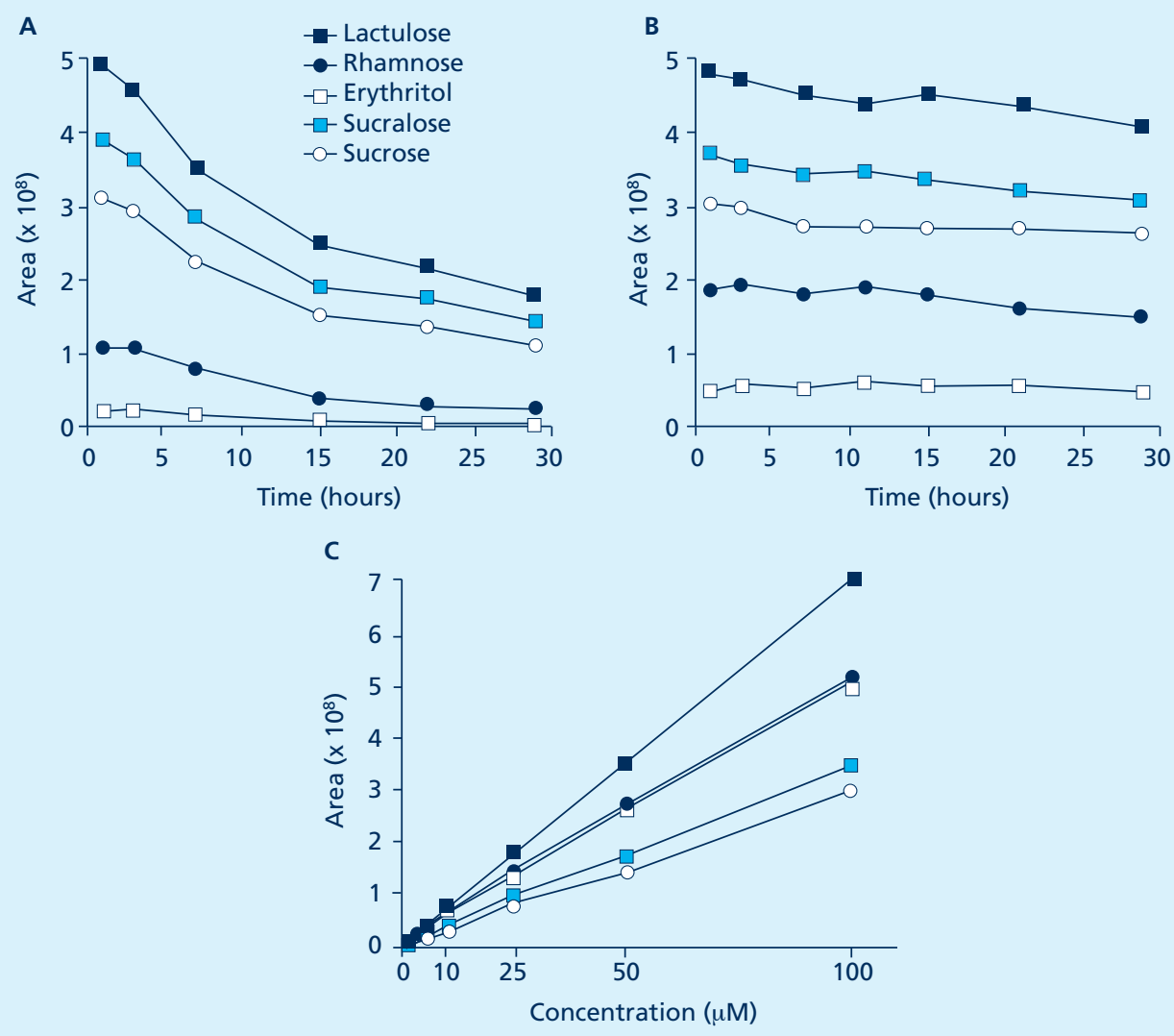

Figure 2: Plasma deproteinization techniques and calibration curves

A) The application of SSA for deproteinization decreases signal in time due to contamination of the MS. B) Cutoff filters provide sufficient plasma deproteinization and stable MS signal strength. C) Calibration curves of target sugar are linear up to $1000 \mu \mathrm{mol} / \mathrm{L}$. Equations and $\mathrm{R}^{2}$ are given in the text.

$\mathrm{mm} \times 7.8 \mathrm{~mm}$ ID) could sufficiently separate the sugars. Therefore, due to the required column dimensions necessary for adequate separation, we had to sacrifice analysis time. To optimize chromatographic separation, the influence of column temperature on the chromatograms was determined in the range of 10 to $90^{\circ} \mathrm{C}$. As expected, peak symmetry and separation of especially the monosaccharides improved at higher temperatures. Since a temperature-dependent instability has been described for disaccharides in solutions (20), we studied whether this decay of disaccharides also occurred on the column above $30^{\circ} \mathrm{C}$. In an attempt to reduce the temperature-dependent instability, we reduced the retention times by applying a shorter IOA-2000 8 $\mu \mathrm{m}$ column (150 mm x $6.5 \mathrm{~mm}$ ID). Unfortunately, even though this column was filled with smaller ion-exchange particles, it did not provide sufficient separation of the sugar probes, as 
Part II - Chapter 5

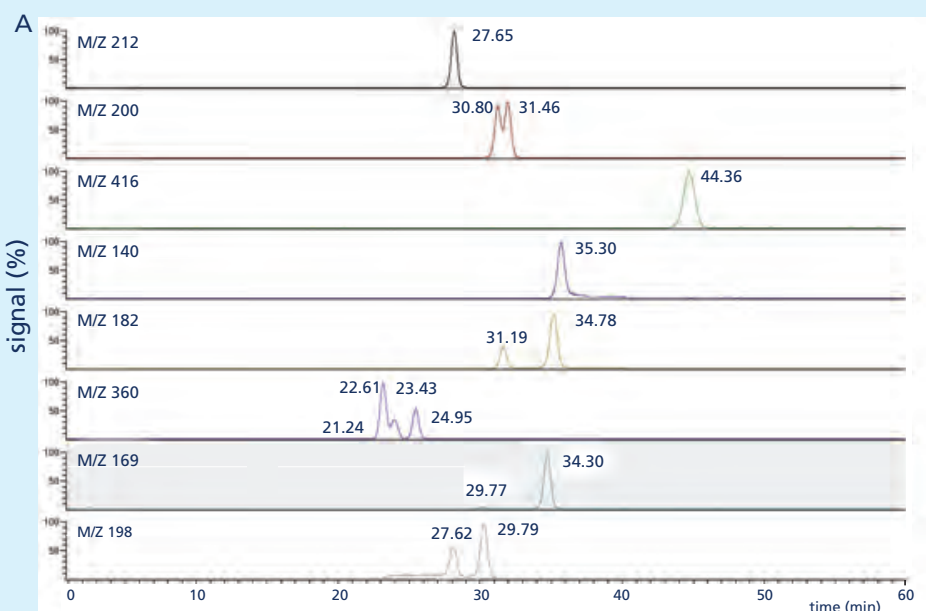

3-OMG (27.65)

mannitol (30.80), sorbitol (31.46)

sucralose (44.36)

erythritol (35.30)

rhamnose (31.19),

fucose (34.78)

sucrose (21.24)

maltose (22.61),

lactose (23.43),

lactulose (24.95)

xylose (29.77),

xylitol (34.30)

glucose (27.62),

mannose (29.79)

B
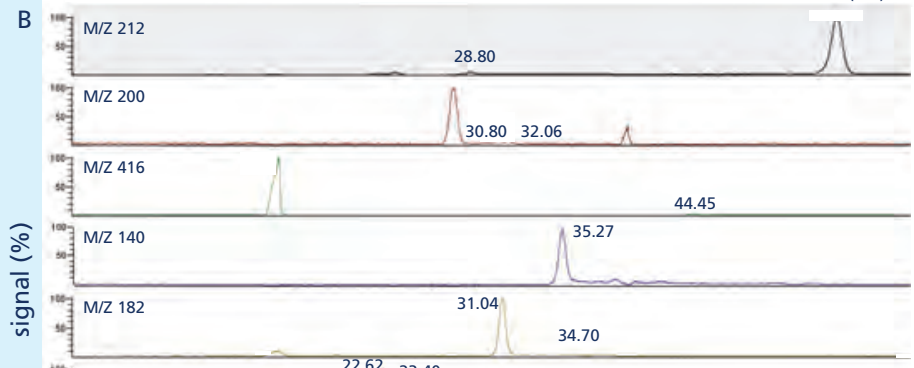

\section{3-OMG (28.80)}

mannitol (30.80), sorbitol (32.06)

sucralose (44.45)

erythritol (35.27)

rhamnose (31.04),

fucose (34.70)

sucrose (22.18),

maltose (22.62)

lactose (23.40),

lactulose (24.93)

xylose (29.77)

xylitol (34.23)

glucose (27.60),

mannose (29.77)

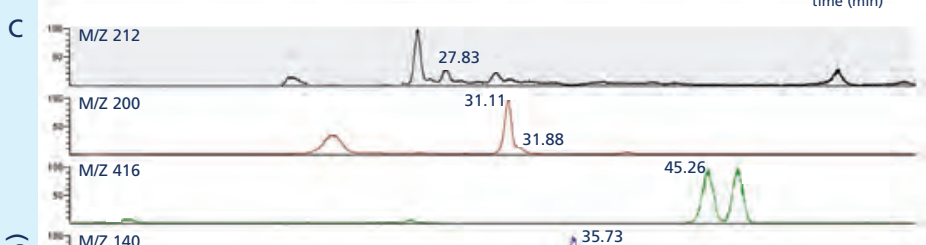

3-OMG (27.83)

mannitol (31.11) sorbitol (31.88)

sucralose (45.26)

erythritol (35.73)

rhamnose (31.64), fucose (35.16)

sucrose (22.16)

maltose (22.91),

lactose (23.80),

lactulose (25.26)

xylose (29.66),

xylitol (34.61)

glucose (27.96),

mannose (29.97) 
was the case when the larger IOA-1000 column was used. Therefore, we continued to use the IOA-1000 column, and the column temperature was set to $30^{\circ} \mathrm{C}$.

To enable the analysis of plasma samples, protein had to be removed. For this purpose, two procedures were evaluated, i.e. addition of SSA and the application of cut-off filters. Addition of SSA resulted in a rapid decrease of the MS signal (Figure $2 A$ ), whereas the application of cutoff filters resulted in a stable MS signal over time (Figure $2 B$ ). Therefore the latter procedure was applied.

Next, we studied the influence of solvent-pH and solvent-anion. Application of a stronger acid like TCA gave a lower solvent-pH, thereby improving peak symmetry and the monosaccharide ionization response, but decreasing the disaccharide ionization response, probably due to enhanced decomposition. Application of a weaker acid, like formic acid, enhanced the disaccharide response, but strongly reduced the monosaccharide response. Therefore, we compromised to a solvent containing $20 \mathrm{mmol} / \mathrm{L}$ formic acid and $10 \mathrm{mmol} / \mathrm{L} \mathrm{TCA}$, resulting in satisfactory separation and peak symmetry for all target sugar probes (Figure 3). Accumulation of buffer salts on the spray shield was minimized by flushing the electrospray source in-between runs with a mixture of methanol/water $(15 / 85 \mathrm{v} / \mathrm{v})$, delivered by a third pump at a flow rate of $0.4 \mathrm{~mL} / \mathrm{min}$, resulting in a stable run-to-run response for all components. Every analytical run started with analysis of the standard from the described standard batch. If the results were similar to the previous results for the standard batch, i.e. if the variation coefficient between standard results was less than $2 \%$, sample analysis was initiated. After each set of 5 samples (either urinary or plasma), a standard was injected for external standard calibration.

\section{Optimizing of MS signal}

The formation of ammonium adducts was initiated by mixing the column effluent with a solution of $30 \mathrm{mmol} / \mathrm{L}$ ammonia dissolved in methanol-water $(20 / 80 \mathrm{v} / \mathrm{v})$, delivered to the system through a second pump that was set at a flow rate of $0.15 \mathrm{~mL} / \mathrm{min}$. The methanol concentration was crucial to obtain maximal signal intensity (26). Alternative organic solvents such as acetonitrile or isopropanol only increased the MS signal marginally. The concentration of ammonia in such solutions was less important. An ammonia concentration of $30 \mathrm{mmol} / \mathrm{L}$ gave the best results. The MS system was set to a high-flow situation, by infusing the sugars through a T-piece into the combined ionization solvent-column effluent solution. Maximal signal intensity was obtained with the following settings: sheath gas 99 (arbitrary) units, auxiliary gas 30 units, spray voltage $4.8 \mathrm{kV}$, heated capillary temperature $220^{\circ} \mathrm{C}$, capillary voltage $44 \mathrm{~V}$ and tube lens: $100 \mathrm{~V}$.

Figure 3 (page 86): The molecular mass-based separation of sugar probes is obtained by chromatography A) Aqueous standard $(250 \mu \mathrm{mol} / \mathrm{L})$. B) Plasma sample obtained $1 \mathrm{~h}$ after oral intake of the sugar test mix. C) Urine sample obtained $1 \mathrm{~h}$ after oral intake of the sugar test mix. For each panel, component names and mass to charge ratios of the monitored $\left[\mathrm{M}+\mathrm{NH}_{4}\right]^{+}$ions $(\mathrm{m} / \mathrm{z}$ values) are provided. 
Table 1: Comparison of calibration curve equations of sugar permeability probes spiked in water, plasma and urine*

\begin{tabular}{llcl} 
& \multicolumn{1}{c}{ Aqueous } & Plasma & Urine \\
Sucrose & $\mathrm{y}=\left(2.9 \times 10^{4}\right) \mathrm{x}+1.8 \times 10^{3}$ & $\mathrm{y}=\left(2.9 \times 10^{4}\right) \mathrm{x}+2.5 \times 10^{5}$ & $\mathrm{y}=\left(2.8 \times 10^{4}\right) \times+2.5 \times 10^{5}$ \\
Lactulose & $\mathrm{y}=\left(6.8 \times 10^{4}\right) \mathrm{x}+2.1 \times 10^{4}$ & $\mathrm{y}=\left(7.0 \times 10^{4}\right) \mathrm{x}+6.4 \times 10^{3}$ & $\mathrm{y}=\left(6.8 \times 10^{4}\right) \mathrm{x}+1.5 \times 10^{4}$ \\
Rhamnose & $\mathrm{y}=\left(5.0 \times 10^{4}\right) \mathrm{x}+9.9 \times 10^{4}$ & $\mathrm{y}=\left(5.2 \times 10^{4}\right) \mathrm{x}+2.0 \times 10^{6}$ & $\mathrm{y}=\left(5.2 \times 10^{4}\right) \mathrm{x}+2.0 \times 10^{6}$ \\
Sucralose & $\mathrm{y}=\left(3.3 \times 10^{4}\right) \mathrm{x}+2.7 \times 10^{4}$ & $\mathrm{y}=\left(3.0 \times 10^{4}\right) \mathrm{x}+2.9 \times 10^{4}$ & $\mathrm{y}=\left(3.1 \times 10^{4}\right) \mathrm{x}+3.7 \times 10^{4}$ \\
Erythritol & $\mathrm{y}=\left(4.9 \times 10^{4}\right) \mathrm{x}+6.5 \times 10^{4}$ & $\mathrm{y}=\left(5.2 \times 10^{4}\right) \mathrm{x}+3.9 \times 10^{5}$ & $\mathrm{y}=\left(5.1 \times 10^{4}\right) \mathrm{x}+5.0 \times 10^{7}$ \\
\hline
\end{tabular}

^Plasma and urine were obtained from 10 fasted, healthy volunteers. Samples were pooled, and spiked with the sugar probes.

\section{Linearity and limit of detection}

Aqueous standards of the sugar probes of interest were run individually and as well as combined in one standard sugar solution. Combination of the sugars in one solution did not change the individual sugar responses (data not shown). The linearity of the detection method was determined by injecting aqueous standards containing all sugars ranging from 1 to $1,000 \mu \mathrm{mol} / \mathrm{L}$. Linear regression analysis of the calibration curves revealed the following slopes, intercepts, and correlation coefficients $\left(\mathrm{R}^{2}\right)$ : Sucrose: $\mathrm{y}=28,777(338) \mathrm{x}+1,787(13,779)$ with $\mathrm{R}^{2}=0.9992$. Lactulose: $\mathrm{y}=68,418(240) \mathrm{x}+21,190(10,464)$ with $\mathrm{R}^{2}=0.9999$. Rhamnose: $\mathrm{y}=49,822(745) \mathrm{x}$ $+99,218(30,352)$ with $\mathrm{R}^{2}=0.9987$. Erythritol: $\mathrm{y}=49,046(553) \mathrm{x}+65,236(22,535)$ with $\mathrm{R}^{2}=$ 0.9992. Sucralose: $y=33,383(310) x+27,250(12,614)$ with $R^{2}=0.9995$ (data are presented as mean(SD); Figure $2 C$ ). The limit of detection, the concentration which generates a signal-tonoise ratio of $3: 1$, was $0.05 \mu \mathrm{mol} / \mathrm{L}$ for the disaccharides and $0.1 \mu \mathrm{mol} / \mathrm{L}$ for the monosaccharides.

To investigate potential matrix interference on the slope of the calibration curves, different concentrations of sugars were spiked to plasma and urine samples, after which samples were analyzed. The data shown in Table 1, revealed that the slopes of the calibration curves were very similar in different matrices. Hence, external calibration in aqueous solution can be applied safely. Internal standard application was not applied.

\section{Coefficient of variation and recovery}

To determine the coefficient of variation, a urine and plasma sample (obtained $1 \mathrm{~h}$ after the oral intake of the sugar test mix) were analyzed in an alternating fashion with a $250 \mu \mathrm{mol} / \mathrm{L}$ standard in 10 -fold. Coefficients of variation ranged from $2.4 \%$ for sucralose to $4.5 \%$ for rhamnose in the standard, from $1.6 \%$ for rhamnose to $4.3 \%$ for sucrose in urine and from $1.2 \%$ for rhamnose to $8.5 \%$ for lactulose in plasma (Table 2). Recovery data were obtained by spiking aliquots of a plasma pool prepared from aliquots of fasted volunteers, with aqueous standards with increasing sugar concentrations. Calculated recoveries were never below $90 \%$ or above $110 \%$, even at the 
Table 2: Coefficient of variation (CV) of sugar concentration measurements in aqueous standard, urine ${ }^{\star}$, and plasma *

\begin{tabular}{|c|c|c|c|c|c|c|c|c|c|c|}
\hline & \multicolumn{2}{|c|}{ Sucrose } & \multicolumn{2}{|c|}{ Lactulose } & \multicolumn{2}{|c|}{ Rhamnose } & \multicolumn{2}{|c|}{ Sucralose } & \multicolumn{2}{|c|}{ Erythritol } \\
\hline & $\begin{array}{l}\text { Conc. } \\
(\mu \mathrm{mol} / \mathrm{L})\end{array}$ & $\begin{array}{l}\mathrm{CV} \\
(\%)\end{array}$ & $\begin{array}{l}\text { Conc. } \\
(\mu \mathrm{mol} / \mathrm{L})\end{array}$ & $\begin{array}{l}\text { CV } \\
(\%)\end{array}$ & $\begin{array}{l}\text { Conc. } \\
(\mu \mathrm{mol} / \mathrm{L})\end{array}$ & $\begin{array}{l}\mathrm{CV} \\
(\%)\end{array}$ & $\begin{array}{l}\text { Conc. } \\
(\mu \mathrm{mol} / \mathrm{L})\end{array}$ & $\begin{array}{l}\text { CV } \\
(\%)\end{array}$ & $\begin{array}{l}\text { Conc. } \\
(\mu \mathrm{mol} / \mathrm{L})\end{array}$ & $\begin{array}{l}\mathrm{CV} \\
(\%)\end{array}$ \\
\hline Standard & 250 & 3.1 & 250 & 2.5 & 250 & 4.5 & 250 & 2.4 & 250 & 4.4 \\
\hline Urine & 28.2 & 4.3 & 6.5 & 3.2 & 234.4 & 1.6 & 25.7 & 2.6 & 4199 & 3.8 \\
\hline Plasma & 0.30 & 5.0 & 0.26 & 8.5 & 102.1 & 1.2 & 0.24 & 3.0 & 117.7 & 2.7 \\
\hline
\end{tabular}

lowest spiked concentration (Table 3). In fact, $67 \%$ of the measurements showed a recovery between $95 \%$ and $105 \%$. To investigate the intra-assay accuracy, aliquots of plasma and urine specimens collected after $1 \mathrm{~h}$ intervals after ingestion of the test mix by a healthy volunteer were measured in 6-fold. The results were plotted in time (Figure 4). Both standards and samples were stored at $4^{\circ} \mathrm{C}$ during the analytical process, and a stable response was observed for at least 24 hours at this temperature.

Table 3: Recovery of sugar permeability probes in spiked plasma *

\begin{tabular}{|c|c|c|c|c|c|}
\hline Spike $(\mu \mathrm{mol} / \mathrm{L})$ & Sucrose & Lactulose & Rhamnose & Sucralose & Erythritol \\
\hline 1.0 & 90.1 & 90.1 & 107.2 & 93.9 & 97.1 \\
\hline 2.5 & 97.0 & 97.0 & 107.8 & 94.0 & 100.8 \\
\hline 10.0 & 103.9 & 103.9 & 106.3 & 90.7 & 101.2 \\
\hline 25.0 & 101.6 & 101.6 & 102.0 & 93.2 & 105.0 \\
\hline 50.0 & 100.4 & 100.4 & 99.9 & 90.6 & 105.3 \\
\hline 100.0 & 99.1 & 99.1 & 104.9 & 91.8 & 105.3 \\
\hline
\end{tabular}

$\star$ Plasma was obtained from 10 fasted, healthy volunteers. Plasma was pooled, and spiked with the sugar probes. Recovery (\%) is presented.

\section{Background sugar concentrations in plasma and urine}

The optimized chromatography enabled the separation of all sugar probes. In addition, a number of components were found in both urine and plasma samples, with molecular masses similar to the target sugars. While most of these components eluted from the column at different time points, some of the components had elution times close to our sugar probes, thereby interfering with the quantification of the sugar probes. Most components could be identified by injecting a range of sugar components in the chromatographic column and comparing the chromatograms of these sugars with the chromatograms of the interfering components (Figure $3 B$ and $C$ ).

In addition, we observed baseline sugar concentrations both in plasma and urine samples of individuals who had been deprived from food and drinks for $10 \mathrm{~h}$. To investigate the relevance of these baseline levels in relation to the increase in concentration caused by oral intake of the test mix, we determined inter-individual and intra-individual differences in 10 volunteers, measured at 4 different days (Supplementary tables). No plasma baseline concentrations of the 

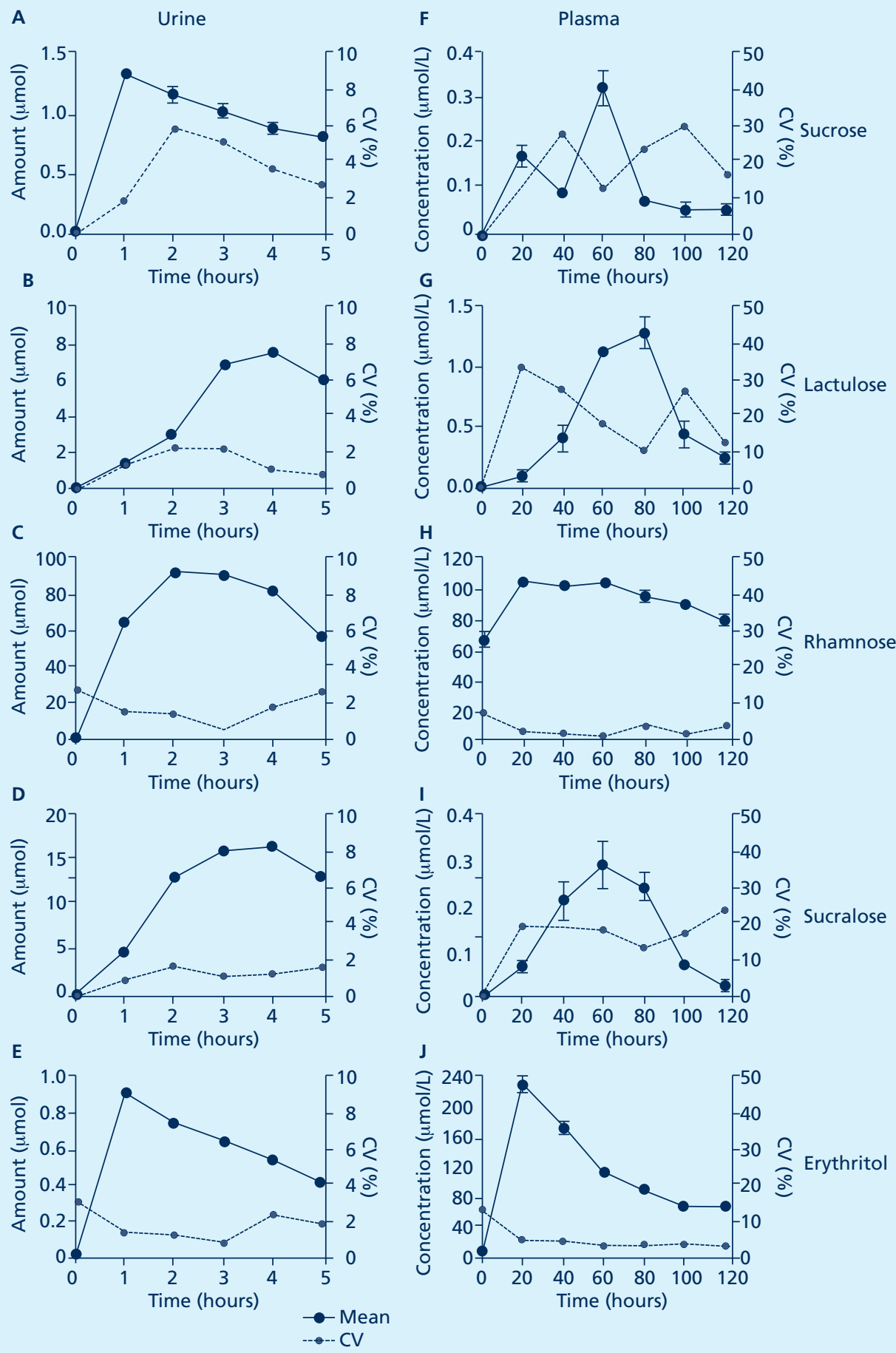
disaccharides lactulose and sucralose were found, while we did observe baseline concentrations of monosaccharide rhamnose in plasma. These concentrations nearly doubled $1 \mathrm{~h}$ after intake of the test mix. In urine, baseline levels ranged from undetectable for lactulose to $724 \mu \mathrm{mol} / \mathrm{L}$ for erythritol (Supplementary Table 1). However, these high baseline concentration for erythritol was low compared to the $21,867 \mu \mathrm{mol} / \mathrm{L}$ measured on average $1 \mathrm{~h}$ after intake of the test mix (data not shown). Therefore, the reported baseline erythritol levels did not interfere with the erythritol measurements necessary for colon permeability assessment (Figure 4).

\section{DISCUSSION}

Various intestinal and systemic diseases are associated with increased GI permeability. Permeability analysis can help to clarify its role in disease. Moreover, for evaluation of interventions directed at preventing increased GI permeability or ameliorating the integrity of the GI epithelium, a sensitive analysis of GI permeability is essential. To improve GI permeability analysis, we developed a new method to measure multiple permeability sugar probes in both plasma and urine.

The new method had to meet three goals: (a) increase the analytical sensitivity, allowing smaller quantities of the sugar probes to be used, (b) measure the classical and additional sugar probes simultaneously in one run, enabling permeability assessment of the stomach, and the small and large intestine, and (c) allow the analysis of the applied sugar probes in both urine and plasma.

Increased analytical sensitivity was obtained by combining isocratic cation-exchange chromatography in combination with MS. Adduct formation, necessary for MS detection, was studied using ammonia and formic acid. Sugar adduct synthesis was much more abundant after addition of ammonia, and therefore sugar-ammonium adducts were analyzed with MS. This approach allowed quantification of all sugar probes of interest in both urine and plasma in one run, with a detection limit of $0.05 \mu \mathrm{mol} / \mathrm{L}$ for disaccharides and $0.10 \mu \mathrm{mol} / \mathrm{L}$ for monosaccharides. These lower limits of detection are significantly lower than previously reported $(7,22)$, including a recent report of Camilleri et al. who found detection limits of $4.98 \mu \mathrm{mol} / \mathrm{L}(1.7$ $\mu \mathrm{g} / \mathrm{mL})$ for disaccharide lactulose and $1.92 \mu \mathrm{mol} / \mathrm{L}(0.035 \mu \mathrm{g} / \mathrm{mL})$ for monosaccharide mannitol using normal phase LC-MS for permeability analysis in urine (7).

Figure 4 (page 90): Amount and intra-assay variation of sugar permeability probes over time in urine and plasma after oral intake of the probes

Amount and intra-assay variation of sugar permeability probes over time in urine and plasma after oral intake of the probes. Amount of sugar probes in urine over time, given as mean and standard deviation (bold lines). In a majority of cases, the standard deviation is too small to indicate. Dotted lines indicate the intra-assay coefficient of variation $(\mathrm{CV})$ from a 6-fold measurement. Urinary excretion of sucrose (A), lactulose (B), rhamnose (C), sucralose (D) and erythritol (E). Plasma concentration of sucrose (F), lactulose (G), rhamnose (H), sucralose (I) and erythritol (J). 
In our study, the increased analytic sensitivity allowed a 5-fold reduction of the oral lactulose dose. The latter made it possible to complement the original dual sugar test mix with additional sugar probes, enabling the assessment of the small and large intestine in one analytical run, with a lower total oral sugar load. Additional sugar probes were selected to assess gastroduodenal (sucrose) and colon permeability (sucralose and erythritol), in addition to small intestinal permeability (lactulose and rhamnose). The disaccharide sucrose is considered a marker of gastroduodenal permeability, since it is short-lived in the upper part of the duodenum due to hydrolysis by sucrose (24). The disaccharide sucralose and the sugar alcohol erythritol are not degraded by human colonic bacteria $(2,14)$ allowing their use as markers of colonic and total GI permeability. Alternatively, assessment of samples (either urine or plasma) collected more frequently than the classical $0-5 \mathrm{~h}$ urinary collection, might reflect permeability of specific parts of the gastrointestinal tract (7). Considering that sugar probes first appear in plasma after permeation through the mucosal epithelium, we aimed to perform plasma-based permeability analysis next to the classical urinary approach. Analysis of plasma samples provided additional analytical challenges. In urine, the ingested sugar probes accumulate, resulting in high urinary sugar concentrations compared to plasma. Another important difference between urine and plasma is the necessity to remove plasma proteins. While at all times, sample preparation was kept as simple as possible to avoid prolongation of the turn-around time, it was necessary to remove these plasma proteins that would otherwise clog the chromatographic column. Whereas conventional plasma deproteinization with SSA resulted in a decrease of the MS signal over time due to contamination of the MS, the application of 3,000 Da cut-off filters proved to be a good and fast alternative. Analysis of frequently sampled plasma samples allows the detection of short-lived changes in GI permeability. Furthermore, combined urinary and plasma analysis might help in the clinical interpretation and understanding of increased or decreased transepithelial permeation of probes.

Analysis of baseline plasma samples revealed MS signals at the $\mathrm{m} / \mathrm{z}$ values of rhamnose and erythritol, strongly suggesting the presence of these monosaccharides in plasma of individuals who had been deprived from food and drinks for $10 \mathrm{~h}$. Such baseline concentrations of sugars and sugar alcohols have been reported in serum and plasma of fasted individuals $(6,9,15)$. These results suggest that monosaccharides may be present in the daily human diet. Small amounts of erythritol have been found in some fruits and red wine for example (23), mannitol can be found in seaweed and other plants (21), and rhamnose naturally occurs in bound form in a variety of plants. Since baseline concentrations of these monosaccharides increased substantially after intake of the probes, these baseline concentrations did not interfere with permeability analysis. Nevertheless, we propose to always collect a baseline sample of each test subject, so that baseline endogenous sugar concentrations can be subtracted from the measured concentrations, thereby giving an accurate reflection of the increase in sugar levels after oral intake of the sugar test mix. The presence of the baseline sugar concentrations implies that a further 
reduction of the oral sugar dose would compromise correct measurement of raised plasma concentrations after intake of the sugar test mix, even though the high analytical sensitivity of the current assay would allow such a reduction. In urine, baseline sugar concentrations did not interfere with the analysis of the sugar permeability probes. Analysis of both plasma and urine revealed the presence of components with identical molecular masses as the target sugars. While most of these products had other retention times than the sugar probes of interest, some components for instance co-eluted with the sucrose peak, thereby hampering accurate measurement of sucrose at the applied oral dose of $1 \mathrm{~g}$. We assume that the application of more sucrose in the test drink would increase the sucrose levels after intake to a higher level, thereby reducing the interfering effect of other components. However, a larger sucrose dose would also increase the osmolarity of our test drink, and since our aim was to improve the sensitivity of the permeability analysis without affecting the GI mucosa through a sugar overload, we chose not to increase the amount of sucrose in our test drink.

The newly developed assay enables the detection of small changes in sugar concentrations in both urine and plasma, allowing accurate assessment of small and large intestinal permeability. The lactulose/rhamnose (L/R) ratio is used as a parameter of small intestinal permeability, while the sucralose/erythritol (S/E) ratio determined in $24 \mathrm{~h}$ urinary collections reflects total GI permeability. Moreover, the combination of the $\mathrm{L} / \mathrm{R}$ and $\mathrm{S} / \mathrm{E}$ ratio might enable specific assessment of colon permeability. Further study is necessary to determine the most suitable time frames to collect urine and plasma samples for the assessment of site-specific gastrointestinal permeability. Furthermore, we are performing validation studies to evaluate the usefulness of our novel approach to assess changes in GI permeability in healthy individuals with and without an oral challenge with indomethacin, after which we plan to conduct further studies to test the applicability of the test in clinical practice. Especially for application in clinical practice, timing of sampling is important to obtain accurate and site-specific information.

In conclusion, we developed a novel analytical approach, based on isocratic cation-exchange LC-MS. This technique enables highly sensitive detection of sugar probes allowing a 5-fold reduction of the oral lactulose dose, and the addition of probes to assess site-specific changes in GI permeability. This assay, applicable to urine and plasma samples, may prove useful in clinical practice for screening, prognosis estimation, and treatment decisions in conditions with suspected gut dysfunction. 
Supplementary Table 1: Baseline concentrations of sugars in urine ${ }^{\star}$ after overnight fast.

\begin{tabular}{|c|c|c|c|c|c|c|c|c|c|c|}
\hline \multirow[t]{2}{*}{ Individual } & \multicolumn{2}{|c|}{$\begin{array}{l}\text { Sucrose } \\
(\mu \mathrm{mol} / \mathrm{L})\end{array}$} & \multicolumn{2}{|c|}{$\begin{array}{l}\text { Lactulose } \\
(\mu \mathrm{mol} / \mathrm{L})\end{array}$} & \multicolumn{2}{|c|}{$\begin{array}{c}\text { Rhamnose } \\
(\mu \mathrm{mol} / \mathrm{L})\end{array}$} & \multicolumn{2}{|c|}{$\begin{array}{c}\text { Sucralose } \\
(\mu \mathrm{mol} / \mathrm{L})\end{array}$} & \multicolumn{2}{|c|}{$\begin{array}{c}\text { Erythritol } \\
(\mu \mathrm{mol} / \mathrm{L})\end{array}$} \\
\hline & Mean & (SEM) & Mean & (SEM) & Mean & (SEM) & Mean & (SEM) & Mean & (SEM) \\
\hline 1 & 77.1 & $(24.7)$ & 0.7 & $(0.7)$ & 46.5 & (4.7) & 0.6 & $(0.6)$ & 415.7 & $(62.0)$ \\
\hline 2 & 86.4 & $(25.2)$ & 0.7 & $(0.7)$ & 265.6 & $(109.5)$ & 0.0 & $(0.0)$ & $1,041.4$ & $(247.1)$ \\
\hline 3 & 109.3 & $(34.8)$ & 0.0 & $(0.0)$ & 87.3 & (18.4) & 0.0 & $(0.0)$ & 580.2 & (105.8) \\
\hline 4 & 21.6 & $(4.3)$ & 0.0 & $(0.0)$ & 28.6 & $(10.9)$ & 1.3 & $(0.7)$ & 416.1 & (159.5) \\
\hline 5 & 75.6 & $(22.0)$ & 1.3 & $(0.8)$ & 141.3 & (45.1) & 0.0 & $(0.0)$ & 987.7 & (197.0) \\
\hline 6 & 51.1 & $(4.7)$ & 0.2 & $(0.2)$ & 55.5 & $(20.6)$ & 21.1 & $(20.4)$ & $1,004.3$ & (227.5) \\
\hline 7 & 68.6 & $(44.8)$ & 0.7 & $(0.7)$ & 62.0 & (13.5) & 42.9 & (33.4) & 958.8 & (636.1) \\
\hline 8 & 15.1 & $(4.0)$ & 0.2 & $(0.2)$ & 70.6 & (2.4) & 3.9 & (3.9) & $1,063.5$ & (136.0) \\
\hline 9 & 50.0 & $(20.2)$ & 1.2 & $(0.8)$ & 93.4 & $(57.5)$ & 0.0 & $(0.0)$ & 221.1 & (47.4) \\
\hline 10 & 61.4 & (33.8) & 0.0 & $(0.0)$ & 35.0 & (13.0) & 0.0 & $(0.0)$ & 555.0 & $(297.2)$ \\
\hline Total & 61.6 & $(9.1)$ & 0.5 & $(0.2)$ & 88.6 & $(22.2)$ & 7.0 & $(4.5)$ & 724.4 & (100.6) \\
\hline
\end{tabular}

*Urine was obtained from 10 fasted, healthy volunteers at 4 different test days. Sugar concentrations are measured in these samples and depicted as mean with standard error of mean (SEM).

Supplementary Table 2: Baseline concentrations of sugars in plasma * after overnight fast.

\begin{tabular}{cccccccccrrr} 
Individual & $\begin{array}{c}\text { Sucrose } \\
(\mu \mathrm{mol} / \mathrm{L})\end{array}$ & $\begin{array}{c}\text { Lactulose } \\
(\mu \mathrm{mol} / \mathrm{L})\end{array}$ & $\begin{array}{c}\text { Rhamnose } \\
(\mu \mathrm{mol} / \mathrm{L})\end{array}$ & $\begin{array}{c}\text { Sucralose } \\
(\mu \mathrm{mol} / \mathrm{L})\end{array}$ & $\begin{array}{c}\text { Erythritol } \\
(\mu \mathrm{mol} / \mathrm{L})\end{array}$ \\
\hline & Mean & $(\mathrm{SEM})$ & Mean & $(\mathrm{SEM})$ & Mean & $(\mathrm{SEM})$ & Mean & $(\mathrm{SEM})$ & Mean & $(\mathrm{SEM})$ \\
1 & 2.9 & $(1.1)$ & 0.1 & $(0.1)$ & 42.7 & $(7.0)$ & 0.1 & $(0.1)$ & 13.2 & $(3.3)$ \\
2 & 2.9 & $(1.2)$ & 0.1 & $(0.1)$ & 51.4 & $(8.8)$ & 0.1 & $(0.1)$ & 7.9 & $(0.5)$ \\
3 & 1.5 & $(0.4)$ & 0.0 & $(0.0)$ & 75.2 & $(6.1)$ & 0.0 & $(0.0)$ & 4.9 & $(0.5)$ \\
4 & 8.7 & $(7.1)$ & 0.1 & $(0.1)$ & 56.9 & $(4.6)$ & 0.1 & $(0.1)$ & 13.4 & $(2.0)$ \\
5 & 1.0 & $(0.4)$ & 0.0 & $(0.0)$ & 51.4 & $(7.4)$ & 0.0 & $(0.0)$ & 5.1 & $(1.2)$ \\
6 & 2.1 & $(0.3)$ & 0.0 & $(0.0)$ & 70.7 & $(10.4)$ & 0.3 & $(0.2)$ & 10.4 & $(2.7)$ \\
7 & 5.9 & $(3.7)$ & 0.0 & $(0.0)$ & 84.4 & $(15.9)$ & 0.0 & $(0.0)$ & 6.2 & $(0.9)$ \\
8 & 1.4 & $(0.1)$ & 0.0 & $(0.0)$ & 95.6 & $(9.5)$ & 0.1 & $(0.1)$ & 6.8 & $(1.5)$ \\
9 & 3.8 & $(0.9)$ & 0.0 & $(0.0)$ & 63.7 & $(8.4)$ & 0.0 & $(0.0)$ & 8.4 & $(1.3)$ \\
10 & 0.9 & $(0.1)$ & 0.0 & $(0.0)$ & 47.6 & $(4.9)$ & 0.0 & $(0.0)$ & 7.4 & $(0.4)$ \\
\hline Total & 3.1 & $(0.78)$ & 0.0 & $(0.0)$ & 63.9 & $(5.5)$ & 0.1 & $(0.0)$ & 8.4 & $(1.0)$ \\
\hline
\end{tabular}

$\star$ Plasma was obtained from 10 fasted, healthy volunteers at 4 different test days. Sugar concentrations are measured in these samples and depicted as mean with standard error of mean (SEM). 


\section{REFERENCES}

1. Antonio C, Pinheiro C, Chaves MM, Ricardo CP, Ortuno MF, and Thomas-Oates J. Analysis of carbohydrates in Lupinus albus stems on imposition of water deficit, using porous graphitic carbon liquid chromatographyelectrospray ionization mass spectrometry. F Chromatogr A 1187: 111-118, 2008.

2. Arrigoni E, Brouns F, and Amado R. Human gut microbiota does not ferment erythritol. Br f Nutr 94: 643646, 2005.

3. Besselink MG, van Santvoort HC, Renooij W, de Smet MB, Boermeester MA, Fischer K, Timmerman HM, Ahmed Ali U, Cirkel GA, Bollen TL, van Ramshorst B, Schaapherder AF, Witteman BJ, Ploeg RJ, van Goor H, van Laarhoven CJ, Tan AC, Brink MA, van der Harst E, Wahab PJ, van Eijck CH, Dejong CH, van Erpecum $\mathrm{KJ}$, Akkermans LM, and Gooszen HG. Intestinal barrier dysfunction in a randomized trial of a specific probiotic composition in acute pancreatitis. Annals of surgery 250: 712-719, 2009.

4. Bjarnason I, MacPherson A, and Hollander D. Intestinal permeability: an overview. Gastroenterology 108: 1566$1581,1995$.

5. Bjarnason I, Maxton D, Reynolds AP, Catt S, Peters TJ, and Menzies IS. Comparison of four markers of intestinal permeability in control subjects and patients with coeliac disease. Scand F Gastroenterol 29: 630-639, 1994.

6. Brown LD, Cavalli C, Harwood JE, Casadei A, Teng CC, Traggiai C, Serra G, Bevilacqua G, and Battaglia FC. Plasma concentrations of carbohydrates and sugar alcohols in term newborns after milk feeding. Pediatric research 64: 189-193, 2008.

7. Camilleri M, Nadeau A, Lamsam J, Nord SL, Ryks M, Burton D, Sweetser S, Zinsmeister AR, and Singh R. Understanding measurements of intestinal permeability in healthy humans with urine lactulose and mannitol excretion. Neurogastroenterol Motil 22: e15-26, 2010.

8. Cobden I, Rothwell J, and Axon AT. Intestinal permeability and screening tests for coeliac disease. Gut 21: 512 $518,1980$.

9. Cox MA, Lewis KO, and Cooper BT. Measurement of small intestinal permeability markers, lactulose, and mannitol in serum: results in celiac disease. Digestive diseases and sciences 44: 402-406, 1999.

10. Dastych M, Dastych M, Jr., Novotna H, and Cihalova J. Lactulose/mannitol test and specificity, sensitivity, and area under curve of intestinal permeability parameters in patients with liver cirrhosis and Crohn's disease. Digestive diseases and sciences 53: 2789-2792, 2008.

11. McIntosh TS, Davis HM, and Matthews DE. A liquid chromatography-mass spectrometry method to measure stable isotopic tracer enrichments of glycerol and glucose in human serum. Anal Biochem 300: 163-169, 2002.

12. Miki K, Butler R, Moore D, and Davidson G. Rapid and simultaneous quantification of rhamnose, mannitol, and lactulose in urine by HPLC for estimating intestinal permeability in pediatric practice. Clin Chem 42: 71 $75,1996$.

13. Oktedalen O, Lunde OC, Opstad PK, Aabakken L, and Kvernebo K. Changes in the gastrointestinal mucosa after long-distance running. Scand $\mathcal{F}$ Gastroenterol 27: 270-274, 1992.

14. Roberts A, Renwick AG, Sims J, and Snodin DJ. Sucralose metabolism and pharmacokinetics in man. Food Chem Toxicol 38 Suppl 2: S31-41, 2000.

15. Roboz J, Kappatos DC, Greaves J, and Holland JF. Determination of polyols in serum by selected ion monitoring. Clin Chem 30: 1611-1615, 1984.

16. Rogatsky E, Tomuta V, and Stein DT. LC/MS quantitative study of glucose by iodine attachment. Anal Chim Acta 591: 155-160, 2007.

17. Rombeau JL, and Takala J. Summary of round table conference: gut dysfunction in critical illness. Intensive care medicine 23: 476-479, 1997.

18. Rooyakkers DR, van Eijk HM, and Deutz NE. Simple and sensitive multi-sugar-probe gut permeability test by high-performance liquid chromatography with fluorescence labelling. F Chromatogr A 730: 99-105, 1996.

19. Sandek A, Bauditz J, Swidsinski A, Buhner S, Weber-Eibel J, von Haehling S, SchroedlW, Karhausen T, Doehner W, Rauchhaus M, Poole-Wilson P, Volk HD, Lochs H, and Anker SD. Altered intestinal function in patients with chronic heart failure. J Am Coll Cardiol 50: 1561-1569, 2007.

20. Slimestad R, and Vagen IM. Thermal stability of glucose and other sugar aldoses in normal phase high performance liquid chromatography. F Chromatogr A 1118: 281-284, 2006.

21. Song SH, and Vieille C. Recent advances in the biological production of mannitol. Appl Microbiol Biotechnol 84: 55-62, 2009.

22. Spiller RC, Jenkins D, Thornley JP, Hebden JM, Wright T, Skinner M, and Neal KR. Increased rectal mucosal enteroendocrine cells, $\mathrm{T}$ lymphocytes, and increased gut permeability following acute Campylobacter enteritis and in post-dysenteric irritable bowel syndrome. Gut 47: 804-811, 2000.

23. Sreenath K, and Venkatesh YP. Analysis of erythritol in foods by polyclonal antibody-based indirect competitive ELISA. Analytical and bioanalytical chemistry 391: 609-615, 2008. 
24. Sutherland LR, Verhoef M, Wallace JL, Van Rosendaal G, Crutcher R, and Meddings JB. A simple, non-invasive marker of gastric damage: sucrose permeability. Lancet 343: 998-1000, 1994.

25.van Nieuwenhoven MA, Geerling BJ, Deutz NE, Brouns F, and Brummer RJ. The sensitivity of the lactulose/rhamnose gut permeability test. European journal of clinical investigation 29: 160-165, 1999.

26. Wan EC, and Yu JZ. Determination of sugar compounds in atmospheric aerosols by liquid chromatography combined with positive electrospray ionization mass spectrometry. $\mathcal{F}$ Chromatogr A 1107: 175-181, 2006. 


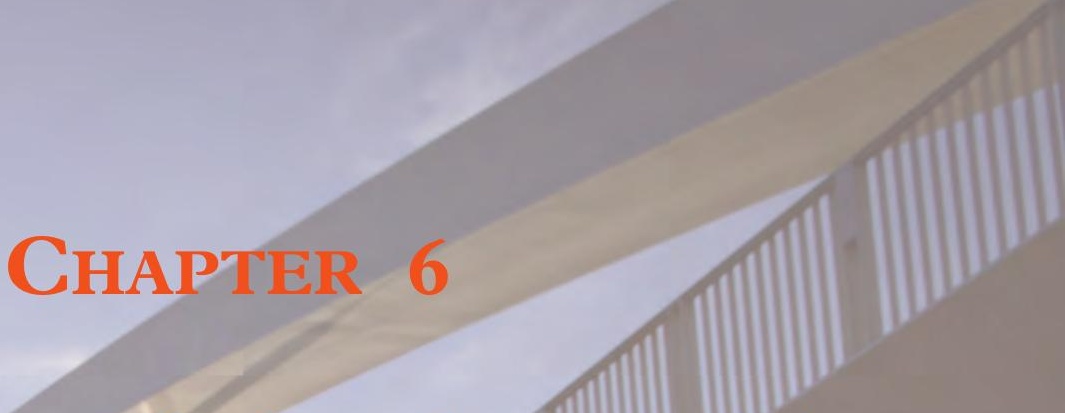

Novel multi-sugar assay for site-specific gastrointestinal permeability analysis: a randomized controlled crossover trial

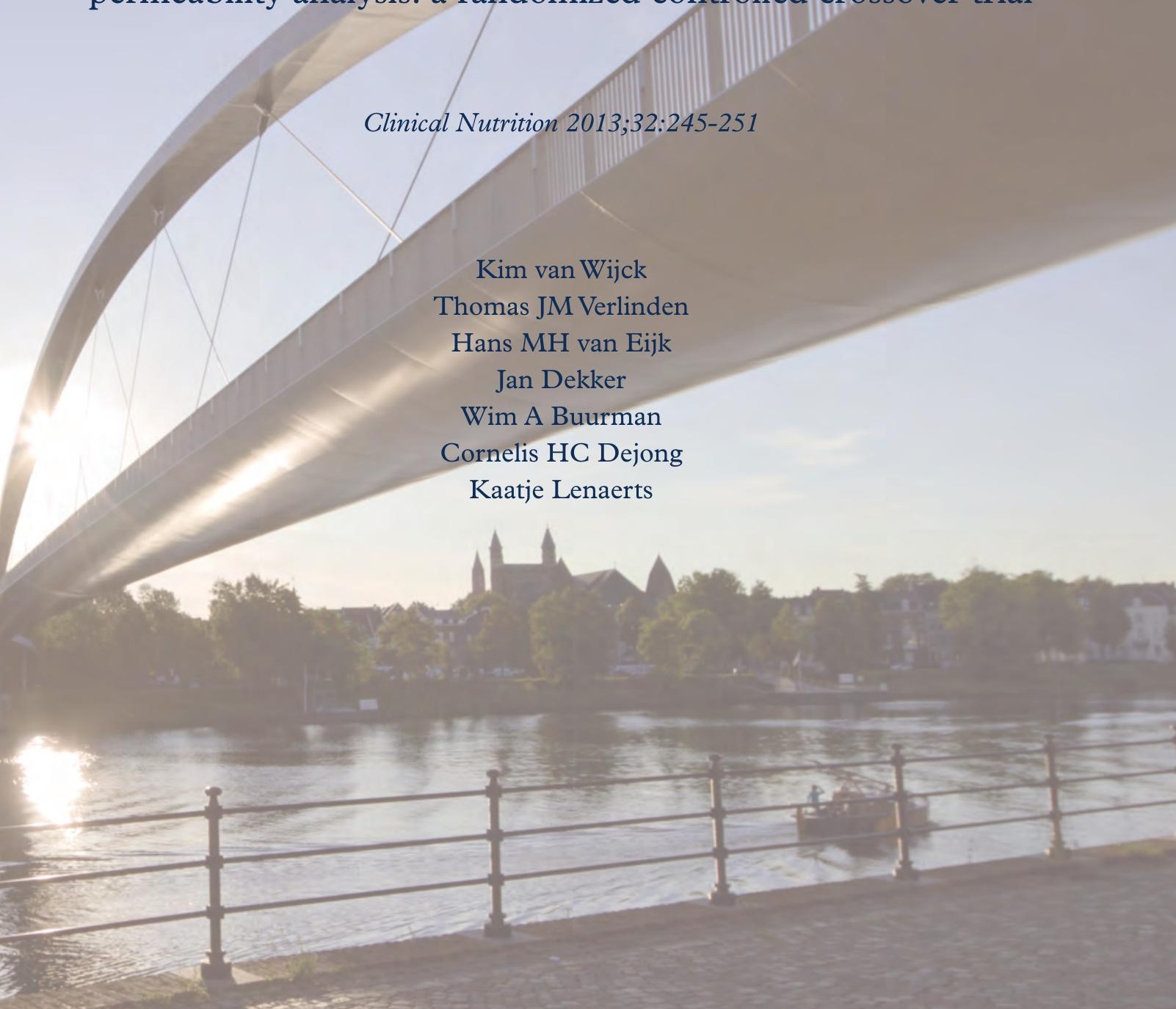




\begin{abstract}
Increased gastrointestinal (GI) permeability is an important hallmark of many conditions, potentially leading to antigen exposure and sepsis. Current permeability tests are hampered by analytical limitations. This study aims to compare the accuracy of our multi-sugar (MS) and the classical dual sugar (DS) test for detection of increased GI permeability.

Ten volunteers received permeability analysis using MS (1 g sucrose, lactulose, sucralose, erythritol, $0.5 \mathrm{~g}$ rhamnose in water) or DS ( $5 \mathrm{~g}$ lactulose, $0.5 \mathrm{~g}$ rhamnose), after indomethacin or placebo. Blood and urine were analyzed by isocratic LC-MS.

MS testing revealed significantly elevated urinary lactulose/rhamnose (L/R) ratios after indomethacin, due to enhanced lactulose excretion $(P<0.01)$ and unaltered rhamnose excretion. The DS test showed increased $\mathrm{L} / \mathrm{R}$ ratios, due to increased lactulose excretion and decreased rhamnose excretion (both $P<0.05$ ). After indomethacin, plasma $\mathrm{L} / \mathrm{R}$ increased in both assays $(P<0.05$ and $P<0.01)$. Urinary and plasma $\mathrm{L} / \mathrm{R}$ ratios correlated significantly. Indomethacin increased sucrose excretion and $0-1 \mathrm{~h}$ sucrose/rhamnose. Colon permeability was unchanged.

In conclusion, sensitive permeability analysis is feasible in plasma and urine using MS or DS test. In contrast to the DS test, monosaccharide excretion is not decreased by the MS test. In short, the MS test provides accurate, site-specific information on gastroduodenal, small, and large intestinal permeability.
\end{abstract}




\section{INTRODUCTION}

Proper gastrointestinal (GI) functioning is crucial for absorption of nutrients, a process facilitated by the extended absorptive area of the GI system. However, its large absorptive area complicates the other main function of the intestine, which is to preserve an effective barrier between internal and external environment. When epithelial integrity is compromised, GI permeability increases, rendering the host more susceptible for potentially harmful elements, such as digestive juices and microbial products $(5,28)$. Increased permeability may initiate pathogenic processes of various intestinal and systemic disorders, and contribute to the development of complications in surgical patients and patients in the $\operatorname{ICU}(5,16,23)$. Therefore, accurate assessment of gut permeability is of great importance (5).

Most techniques to assess human gut permeability rely on the urinary excretion of orally administered test probes. Classically, a disaccharide (usually lactulose) and monosaccharide (mannitol or L-rhamnose) are administered together, and appear in blood and urine after permeating the epithelial barrier (26). The calculated urinary excretion ratio of these probes reflects small intestinal permeability $(5,26)$. We recently developed a detection method that yielded a detection limit of $0.05 \mu \mathrm{mol} / \mathrm{L}$ for disaccharides and $0.10 \mu \mathrm{mol} / \mathrm{L}$ for monosaccharides $(36,37)$, which meant a significant improvement with respect to previous studies reporting limits of 5-30 $\mu \mathrm{mol} / \mathrm{L}$ for disaccharide lactulose and $2-5 \mu \mathrm{mol} / \mathrm{L}$ for monosaccharide mannitol. This lowering of the detection limit enabled a 5-fold reduction of the oral lactulose load and allowed concomitant expansion with probes to assess gastroduodenal and colonic permeability simultaneously with small permeability assessment.

The current randomized, controlled, crossover trial was performed to validate the novel sitespecific MS permeability test in healthy volunteers, challenged with the non-steroidal antiinflammatory drug (NSAID) indomethacin to increase GI permeability $(6,7,30,31)$.

\section{MATERIALS AND METHODS}

This study was approved by the medical ethical committee of Maastricht University Medical Center and conducted in Maastricht, according to the Declaration of Helsinki (revised version, October 2008, Seoul). The study is registered at US National Library of Medicine (http://www.clinicaltrials.gov, NCT00943345).

\section{Materials}

The MS mix consisted of $1 \mathrm{~g}$ sucrose (Van Gilse, Dinteloord, the Netherlands), $1 \mathrm{~g}$ lactulose (Centrafarm, Etten-Leur, the Netherlands), 1 g sucralose (Brenntag, Sittard, the Netherlands), $1 \mathrm{~g}$ erythritol (Danisco Sweeteners, Copenhagen, Denmark) and $0.5 \mathrm{~g}$ of L-rhamnose (Danisco), 
dissolved in $150 \mathrm{~mL}$ of tap water. The DS mix consisted of $5 \mathrm{~g}$ lactulose and $0.5 \mathrm{~g}$ L-rhamnose. All products were intended and tested for safe oral human consumption by Basic Pharma (Geleen, the Netherlands). The sugar probes were 95.0-99.9\% pure, and were calibrated against analytical grade products for concentration analysis in plasma and urine.

\section{Participants}

Healthy men and women, aged 18-70 years, were selected. Sample size was determined using PS Power and Sample Size Calculations, based on a previous study using indomethacin to increase GI permeability (14). A power calculation showed that a sample size of 10 individuals was required to achieve $80 \%$ power for detection of a statistically significant effect size of 2.5 (standard deviation of 2.5) with an $\alpha$-error of 0.05. Exclusion criteria were reported abdominal complaints assessed by questionnaire prior to inclusion, recent use of medication, history of GI surgery, family history of inflammatory bowel disease, hypersensitivity to NSAID or food products, pregnancy, alcohol or substance misuse, and any other medical condition that could influence the experimental results. Volunteers were informed about the nature and risks of the experiments. Written consent was obtained 5 days before the experiments. Use of medication other than indomethacin was prohibited during the study period.

\section{Pre-test restrictions}

Test subjects were instructed to maintain normal activities of daily living and normal dietary patterns, to record dietary intake, starting 2 days prior the experiments, and to avoid intake of sugars that were part of the MS mix and alcohol, caffeine or spicy products. Participants received a standardized evening meal $(1695 \mathrm{~kJ}$, consisting of $62.6 \mathrm{~g}$ carbohydrate, $18.9 \mathrm{~g}$ protein and 7.9 $\mathrm{g}$ fat) and thereafter fasted overnight. Subjects were allowed to drink water (maximum $200 \mathrm{~mL}$ per hour).

\section{Study design}

The study is randomized placebo-controlled and double-blinded in set-up. The random allocation sequence was established by computer-generated simple randomization. For the randomization process, numbers were assigned to each test situation. All subjects completed 4 days to test basal and indomethacin-induced GI permeability with both tests. Interval between test days was at least 7 days. Study design is depicted in Supplementary Figure 1. Subjects ingested placebo capsules (basal permeability) or indomethacin-filled capsules (Actavis, Baarn, the Netherlands) $75 \mathrm{mg}$ at 22:00 P.M. the day before the test and 50mg at 7:30 A.M. on the test day. Capsules were macroscopically identical. Subjects were tested in fasted state. During the experiments, subjects drank 150-200 mL water or tea without additives per hour to maintain sufficient urinary output for hourly urinary collection. After completion of the $5 \mathrm{~h}$ urinary collection, subjects were given a standardized meal consisting of sandwiches and tea. Following this delayed breakfast, 
subjects were allowed to go home and eat and drink as preferred, with the exception of sugars included in the test mix, alcohol, caffeine and spicy products. After completion of the $24 \mathrm{~h}$ urinary collection the next morning, subjects were allowed to eat and drink without restrictions. Urine and blood were collected and stored as previously described (36).

\section{Analysis of intestinal damage and inflammation}

To assess intestinal damage and inflammation caused by indomethacin, plasma concentrations of human Intestinal Fatty Acid Binding Protein (I-FABP) and calprotectin were determined, respectively, by ELISA, as previously described (36). Both these secondary outcome measures were determined on all experimental days, in each first blood sample of the day $(t=0)$.

\section{Permeability analysis}

Primary outcome measures were urinary sugar excretion rates and sugar permeability ratios for GI permeability assessment. Isocratic ion-exchange HPLC (Model PU-1980 pump, Jasco Easton, MD) with mass spectrometry (Model LTQ XL, Thermo Fisher Scientific, Waltham, MA) was used to determine sugar concentrations in plasma and urine as previously described (37). The following formulas were used:

Urinary sugar excretion $=$ (urine sugar concentration - baseline urine sugar concentration) $\mathrm{x}$ urine volume

Sugar recovery (in \%) = urinary sugar excretion / orally ingested sugar dose x 100

Sugar permeability ratio = sugar excretion (urine) or sugar concentration (plasma) of large probe / sugar excretion or concentration of smaller sugar probe

Sucrose in the MS mix was used for gastroduodenal permeability assessment $(25,32)$. Since this disaccharide is rapidly hydrolyzed by sucrase in the duodenum (9), it is considered specific for the upper GI tract. Lactulose and monosaccharide rhamnose, and the lactulose/rhamnose $(\mathrm{L} / \mathrm{R})$ ratio were used for small intestinal permeability assessment as usual. Since lactulose and rhamnose are degraded in the human colon, two non-degradable probes, sucralose $(12,24)$ and erythritol (15) were used to assess colonic permeability, by calculation of the sucralose/erythritol $(\mathrm{S} / \mathrm{E})$ ratio in $5-24 \mathrm{~h}$ urine.

\section{Statistical analysis}

Statistical analysis was performed using GraphPad Prism (Version 5.00, San Diego, CA). Normality of data was verified by Kolmogorov-Smirnov test. One outlier identified using Grubbs analysis, was excluded from statistical analysis for plasma calprotectin analysis. Area under the curve (AUC) was determined for $\mathrm{L} / \mathrm{R}$ ratios and sugar concentrations. Measurements of basal intestinal permeability and indomethacin-induced permeability were compared using Wilcoxon signed rank test. Receiver Operating Characteristics (ROC) curves were plotted to determine 
cutoff points discriminating between basal and indomethacin-induced permeability. Best cutoff points, defined as the maximum sum of sensitivity and specificity, were reported. Correlations were computed by calculating Spearman correlation coefficients $\left(R_{S}\right)$. Data are presented as mean \pm standard error of the mean (SEM) if normally distributed, or as median (range) of 10 healthy individuals with and without intake of indomethacin. $P<0.05$ was considered statistically significant.

\section{RESULTS}

\section{Participant characteristics}

Ten healthy volunteers ( 5 men, 5 women; mean age $35.2 \pm 5.9$ years) were included in this study and each volunteer performed 4 test days. Baseline characteristics of all participants are shown in Table 1. No GI complaints were experienced during test days or day thereafter.

Table 1: Baseline characteristics of healthy participants

\begin{tabular}{|c|c|}
\hline Characteristics & \\
\hline Group size (no. of volunteers) & 10 \\
\hline Sex (male : female) & $5: 5$ \\
\hline Age (years) & $35.2 \pm 5.9$ \\
\hline Height $(\mathrm{cm})$ & $173 \pm 3.7$ \\
\hline Weight (kg) & $76.2 \pm 3.8$ \\
\hline Body Mass Index $\left(\mathrm{kg} / \mathrm{m}^{2}\right)$ & $25.5 \pm 1.1$ \\
\hline
\end{tabular}

\section{Indomethacin-induced intestinal damage and inflammation}

Plasma I-FABP levels were significantly higher after intake of indomethacin (818 (321-2578) $\mathrm{pg} / \mathrm{ml}$ ), compared to placebo (509 (233-1272) pg/mL; $P<0.001 ;$ Figure $1 A$ ). Indomethacininduced inflammation, determined by plasma calprotectin, was also increased after indomethacin (121 (25-273) ng/mL) compared to placebo (103 (19-176) ng/mL; $P<0.01$; Figure 1B). While both I-FABP and calprotectin levels varied among the test subjects (Figure $1 \mathrm{~A}$ and $1 \mathrm{~B}$ ), the intraindividual values were consistent. Only one subject showed an apparent decrease in plasma calprotectin level after intake of indomethacin; such a decrease was not observed for plasma I-FABP levels. The indomethacin-induced damage and inflammation correlated significantly $\left(\mathrm{R}_{\mathrm{S}}=0.46 ; \mathrm{P}<0.01 ; \mathrm{n}=10\right)$. 


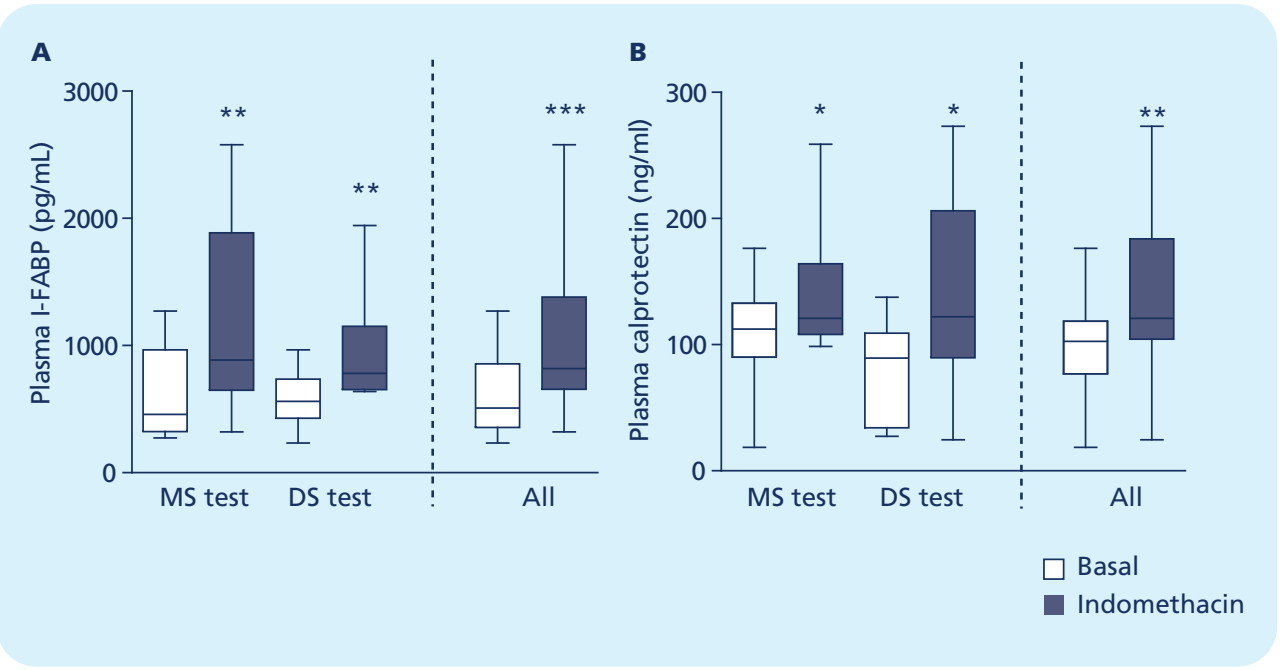

Figure 1: Indomethacin results in small intestinal cell damage and inflammation

A) Plasma I-FABP levels are increased after intake of indomethacin, indicating indomethacin-induced enterocyte damage. B) Plasma calprotectin levels are increased after intake of indomethacin, indicating the presence of indomethacin-induced inflammation. One outlier with a very high plasma calprotectin level $(972 \mathrm{ng} / \mathrm{ml}) \mathrm{after}$ indomethacin on the MS test day was excluded. ( ${ }^{\star} P<0.05,{ }^{\star \star} P<0.01,{ }^{\star \star \star} P<0.001$ compared to basal).

\section{Changes in small intestinal permeability assessed in urine}

Indomethacin significantly increased small intestinal permeability, reflected by increased urinary $\mathrm{L} / \mathrm{R}$ ratios in both permeability tests (Figure 2). Using the MS test, indomethacin resulted in a $0-5 \mathrm{~h} \mathrm{~L} / \mathrm{R}$ ratio of $0.060 \pm 0.011$ compared to the baseline $\mathrm{L} / \mathrm{R}$ ratio of $0.026 \pm 0.004(P<0.05$; Figure $2 A$ ). The $0-5 \mathrm{~h} \mathrm{~L} / \mathrm{R}$ ratio measured with the $\mathrm{DS}$ test was $0.262 \pm 0.034$ after indomethacin, compared to $0.129 \pm 0.014$ at baseline $(P<0.05$; Figure $2 B)$.

Since it was suggested that lactulose influences rhamnose excretion $(17,35)$, we compared absolute urinary excretion of lactulose and rhamnose. Lactulose excretion increased in both tests after intake of indomethacin, but was more pronounced when the MS test was used, showing an increase in the $0-1 \mathrm{~h}, 1-2 \mathrm{~h}$, and $0-5 \mathrm{~h}$ urinary collections (all $P<0.01$; Figure $2 \mathrm{C}$ ). $0-5 \mathrm{~h}$ urinary lactulose excretion was $15.2 \pm 2.1 \mu \mathrm{mol}$ versus $7.5 \pm 1.3 \mu \mathrm{mol}$ at baseline. Lactulose excretion using the DS test increased only in $0-5 \mathrm{~h}(44.7 \pm 8.3 \mu \mathrm{mol}$ versus $32.7 \pm 5.7 \mu \mathrm{mol}$ at baseline; $P<0.05$; Figure $2 D$ ). Interestingly, rhamnose in $0-5 \mathrm{~h}$ urine decreased considerably in this test after indomethacin ( $255 \pm 26 \mu \mathrm{mol}$ versus $185 \pm 15 \mu \mathrm{mol} ; P<0.05$; Figure $2 F$ ), while an opposite observation was done using the MS test $(305 \pm 45 \mu \mathrm{mol}$ at baseline versus $404 \pm 88 \mu \mathrm{mol}$ after indomethacin; Figure 2E). 

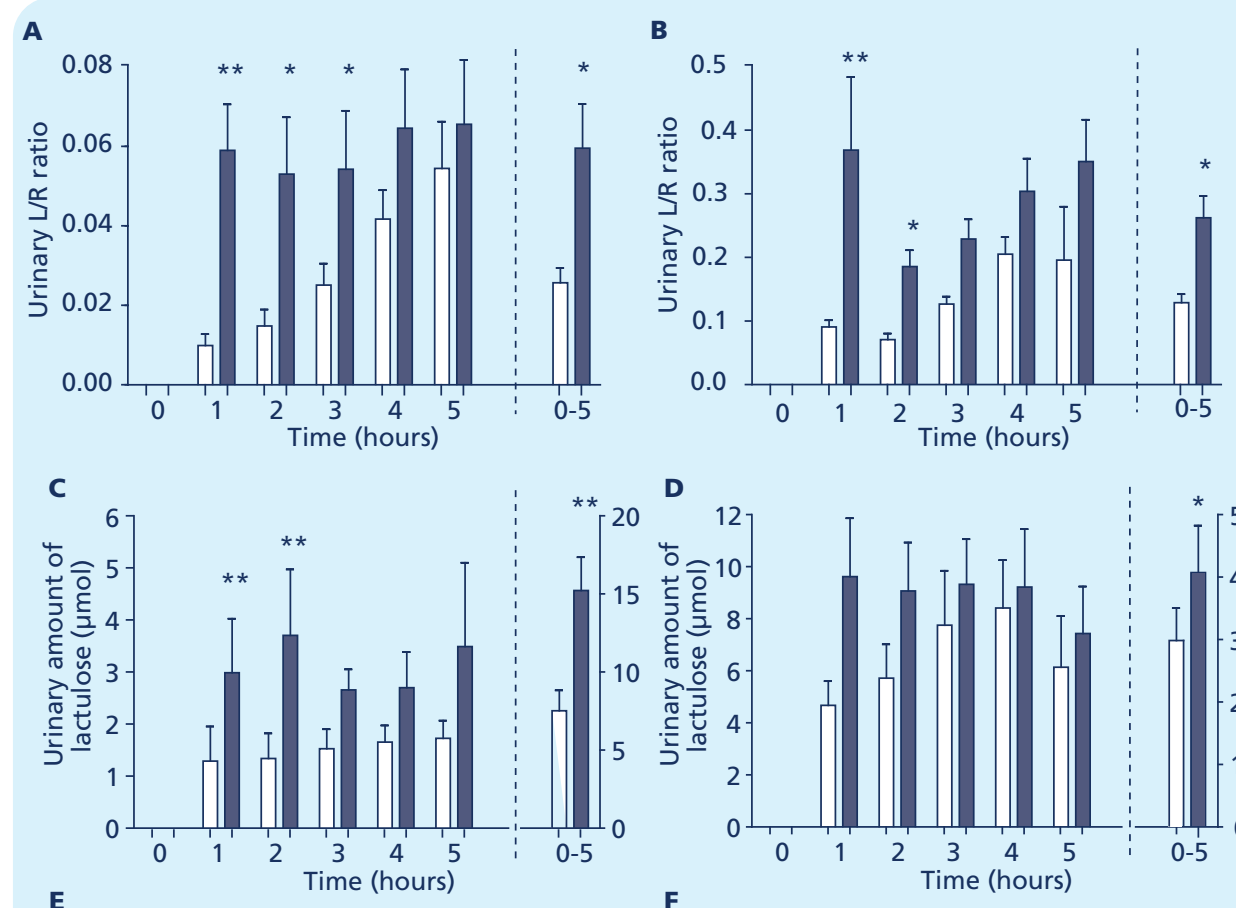

D
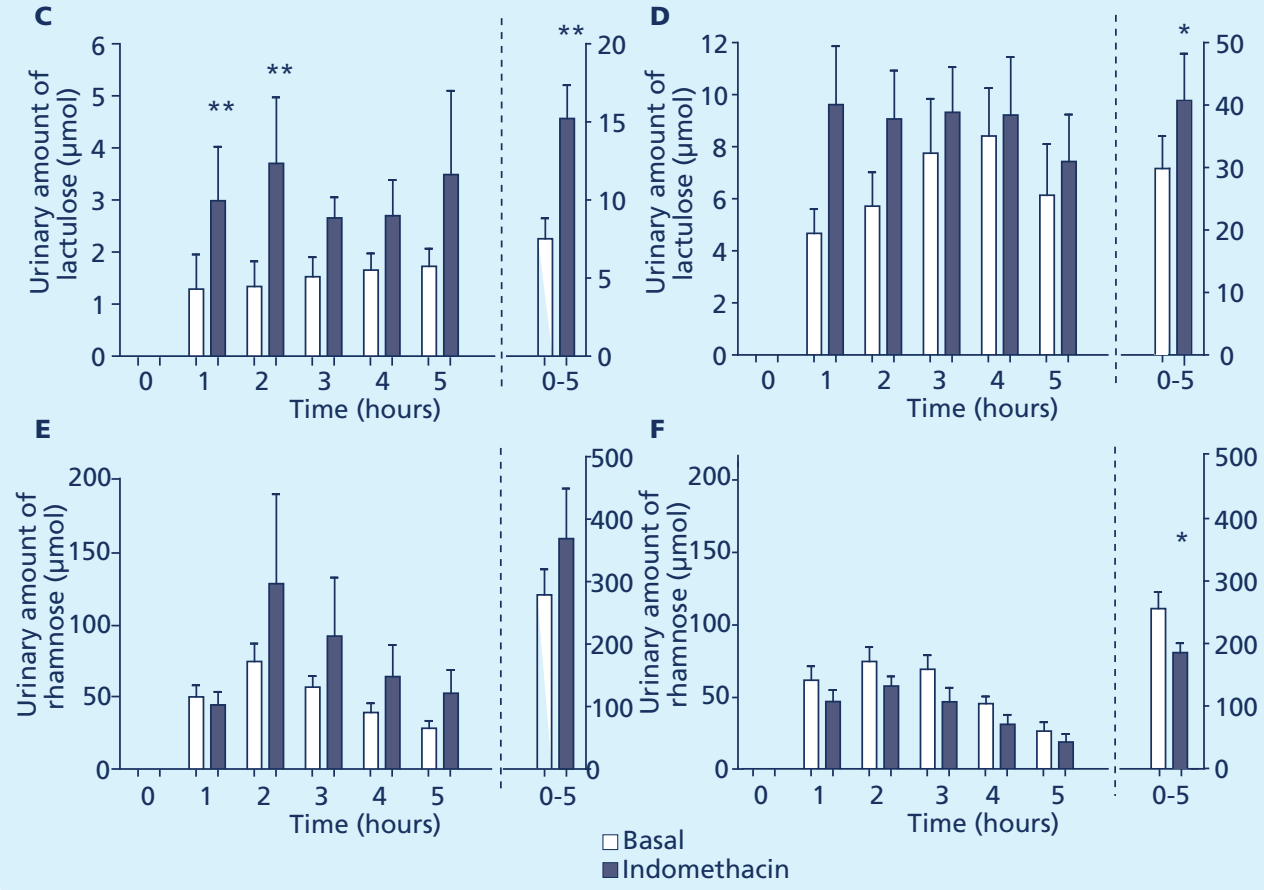

Figure 2: Urinary $L / R$ ratios and excretion rates increase after intake of indomethacin

A) MS test: Indomethacin elevates urinary L/R ratios. B) DS test: Urinary L/R ratios are increased after intake of indomethacin.. Indomethacin increases urinary lactulose excretion, measured by the MS (C) and DS (D) test. E) Urinary excretion of rhamnose is not significantly increased after indomethacin challenge when measured with the MS test. F) Indomethacin decreases urinary rhamnose excretion when the DS test is used for small intestinal permeability analysis. $\left({ }^{\star} P<.05,{ }^{\star}{ }^{\star} P<.01\right.$ compared to basal).

Both tests were evaluated for accuracy to discriminate between basal permeability and experimentally increased permeability. The MS test had a sensitivity and specificity of $100 \%$ in the 0-1h collection, compared to 90 and $89 \%$, respectively, with the DS test (Table 2). Both permeability tests could discriminate indomethacin-induced permeability from basal permeability in 
Table 2: Overall accuracy and cutoff points of both urine-based permeability tests

\begin{tabular}{|c|c|c|c|c|c|}
\hline & $\begin{array}{l}\text { Cutoff } \\
\text { point }\end{array}$ & $\begin{array}{l}\text { Sensitivity } \\
\left(95 \% \mathrm{CI}^{2}\right)\end{array}$ & Specificity & $\mathrm{AUC}^{1}$ & $P$ \\
\hline $\mathrm{L} / \mathrm{R}$ in $\mathrm{MS}$ test ( $0-1 \mathrm{~h}$ urine $)$ & 0.0305 & $100 \%$ & $100 \%$ & $1.00(1.00-1.00)$ & $<0.001$ \\
\hline $\mathrm{L} / \mathrm{R}$ in $\mathrm{DS}$ test ( $0-1 \mathrm{~h}$ urine $)$ & 0.1140 & $90 \%$ & $89 \%$ & $0.97(0.90-1.04)$ & $<0.001$ \\
\hline $\mathrm{L} / \mathrm{R}$ in $\mathrm{MS}$ test ( $0-2 \mathrm{~h}$ urine $)$ & 0.0210 & $90 \%$ & $90 \%$ & $0.88(0.71-1.06)$ & $<0.01$ \\
\hline $\mathrm{L} / \mathrm{R}$ in $\mathrm{DS}$ test $(0-2 \mathrm{~h}$ urine $)$ & 0.1015 & $100 \%$ & $90 \%$ & $0.98(0.96-1.02)$ & $<0.001$ \\
\hline $\mathrm{L} / \mathrm{R}$ in MS test ( $0-5 \mathrm{~h}$ urine $)$ & 0.0450 & $67 \%$ & $100 \%$ & $0.83(0.63-1.04)$ & 0.01 \\
\hline $\mathrm{L} / \mathrm{R}$ in $\mathrm{DS}$ test $(0-5 \mathrm{~h}$ urine $)$ & 0.2510 & $70 \%$ & $60 \%$ & $0.62(0.35-0.88)$ & 0.38 \\
\hline $\mathrm{L} / \mathrm{R}$ in $\mathrm{MS}$ test $(0-1 \mathrm{~h}$ plasma $)$ & 0.045 & $90 \%$ & $70 \%$ & $0.80(0.58-1.02)$ & $<0.05$ \\
\hline $\mathrm{L} / \mathrm{R}$ in $\mathrm{DS}$ test $(0-1 \mathrm{~h}$ plasma $)$ & 0.260 & $80 \%$ & $100 \%$ & $0.86(0.66-1.00)$ & $<0.01$ \\
\hline $\mathrm{L} / \mathrm{R}$ in $\mathrm{MS}$ test $(0-5 \mathrm{~h}$ plasma) & 0.0075 & $41 \%$ & $79 \%$ & $0.57(0.50-0.60)$ & $<0.05$ \\
\hline $\mathrm{L} / \mathrm{R}$ in $\mathrm{DS}$ test $(0-5 \mathrm{~h}$ plasma $)$ & 0.0315 & $57 \%$ & $96 \%$ & $0.85(0.81-0.89)$ & $<0.001$ \\
\hline Sucrose $(0-1 \mathrm{~h}$ urine $)$ & 1.125 & $90 \%$ & $50 \%$ & $0.66(0.41-0.91)$ & 0.23 \\
\hline Sucrose $(0-1 \mathrm{~h}$ plasma $)$ & 1.519 & $70 \%$ & $80 \%$ & $0.73(0.49-0.96)$ & 0.09 \\
\hline $\mathrm{S} / \mathrm{R}(0-1 \mathrm{~h}$ urine $)$ & 0.0565 & $60 \%$ & $90 \%$ & $0.78(0.57-0.99)$ & $<0.05$ \\
\hline
\end{tabular}

0-2h. However, while accuracy of the MS test was still acceptable in 0-5h urine (AUC 0.83, $P=0.01$ ), the DS test was insufficient (AUC $0.62, P=0.38$ ). Urinary recoveries of all applied sugar probes, analyzed by the MS and DS test, are depicted in the Supplementary file as Figure 2.

\section{Changes in small intestinal permeability assessed in plasma}

In line with urine, plasma $\mathrm{L} / \mathrm{R}$ ratios increased in both tests after indomethacin (Figure $3 \mathrm{AB}$ ). $1 \mathrm{~h}$ after intake of the test mix, plasma $\mathrm{L} / \mathrm{R}$ ratios were higher after indomethacin challenge (Figure $3 C D$ ). Interestingly, these ratios determined in $1 \mathrm{~h}$ plasma samples correlated significantly with plasma I-FABP levels determined at baseline $\left(\mathrm{R}_{\mathrm{S}}=0.53 ; P<0.05\right.$ for low-dose lactulose test and $\mathrm{R}_{\mathrm{S}}=0.58 ; P<0.01$ for the high-dose test; Supplementary Figure $\left.3 A B\right)$. Furthermore, plasma $\mathrm{L} / \mathrm{R}$ ratios also correlated significantly with urinary $\mathrm{L} / \mathrm{R}$ ratios determined in both tests $\left(\mathrm{R}_{\mathrm{S}}=0.69\right.$; $P<0.001$ for MS and $R_{S}=0.59 ; P<0.01$ for DS; Supplementary Figure $3 C D$ ), emphasizing the validity of the novel detection method. Both assays could differentiate increased permeability from basal permeability by evaluation of the $1 \mathrm{~h}$ plasma L/R ratio, albeit the DS test did slightly better than the MS test (Table 2). In the following hours, increases in plasma L/R were less pronounced using the latter, while the high-dose test showed elevated $\mathrm{L} / \mathrm{R}$ ratios also at later time points (data not shown).

Plasma sugar concentrations of both tests were studied to find out the origin of the increased plasma L/R ratios. For both assays, significant increases in plasma lactulose concentration were observed in $0-5 \mathrm{~h} \quad(P<0.05$ and $P=0.01)$. Plasma rhamnose levels did not change after indomethacin when the MS test was used, while lower plasma rhamnose concentrations after indomethacin were found using the DS test (data not shown). 
A

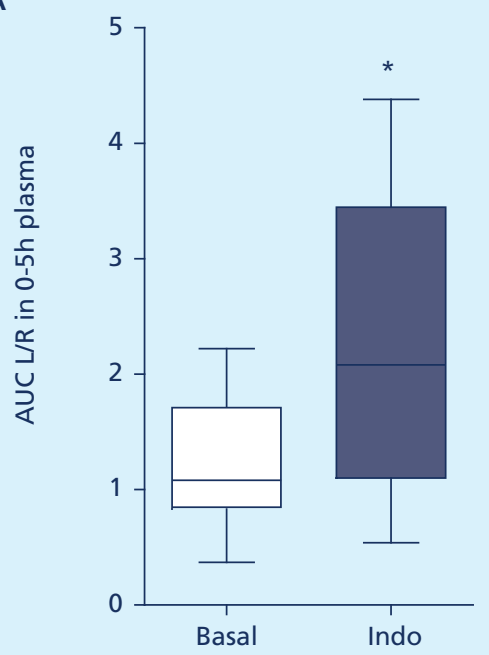

C

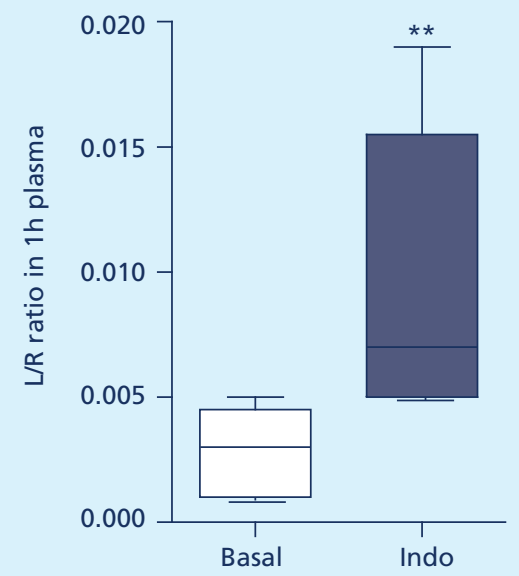

B

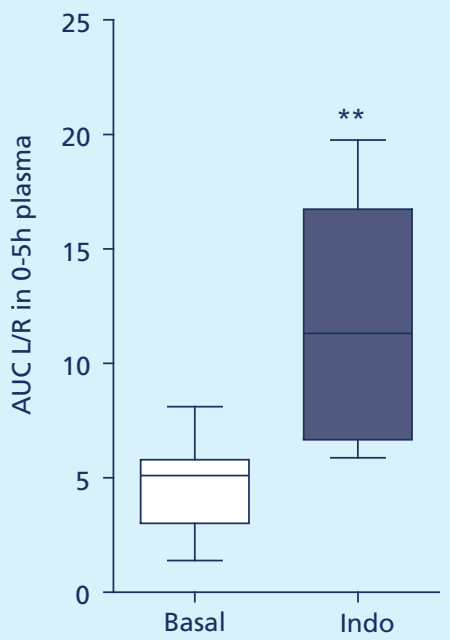

D

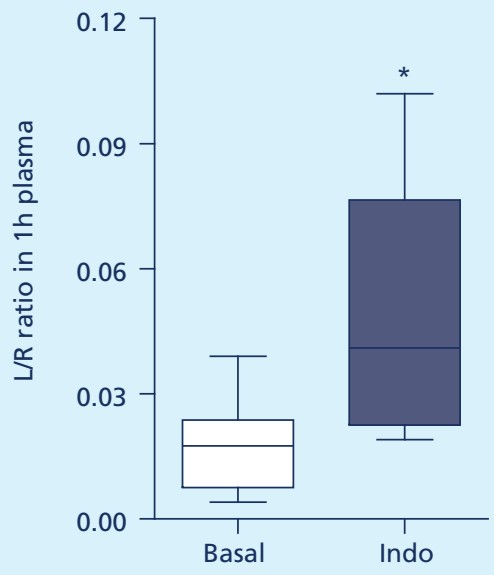

Figure 3: Plasma L/R ratios increase after intake of indomethacin

Indomethacin increased the area under the curve (AUC) of the plasma L/R ratios determined in the $0-5 \mathrm{~h}$ time period measured by the MS test (A) and DS test (B). Plasma L/R ratios $1 \mathrm{~h}$ after intake of the test mix were increased after indomethacin, measured with the MS test (C) and DS test (D). $\left({ }^{\star} P<.05,{ }^{\star}{ }^{\star} P<.01\right.$ compared to basal). 

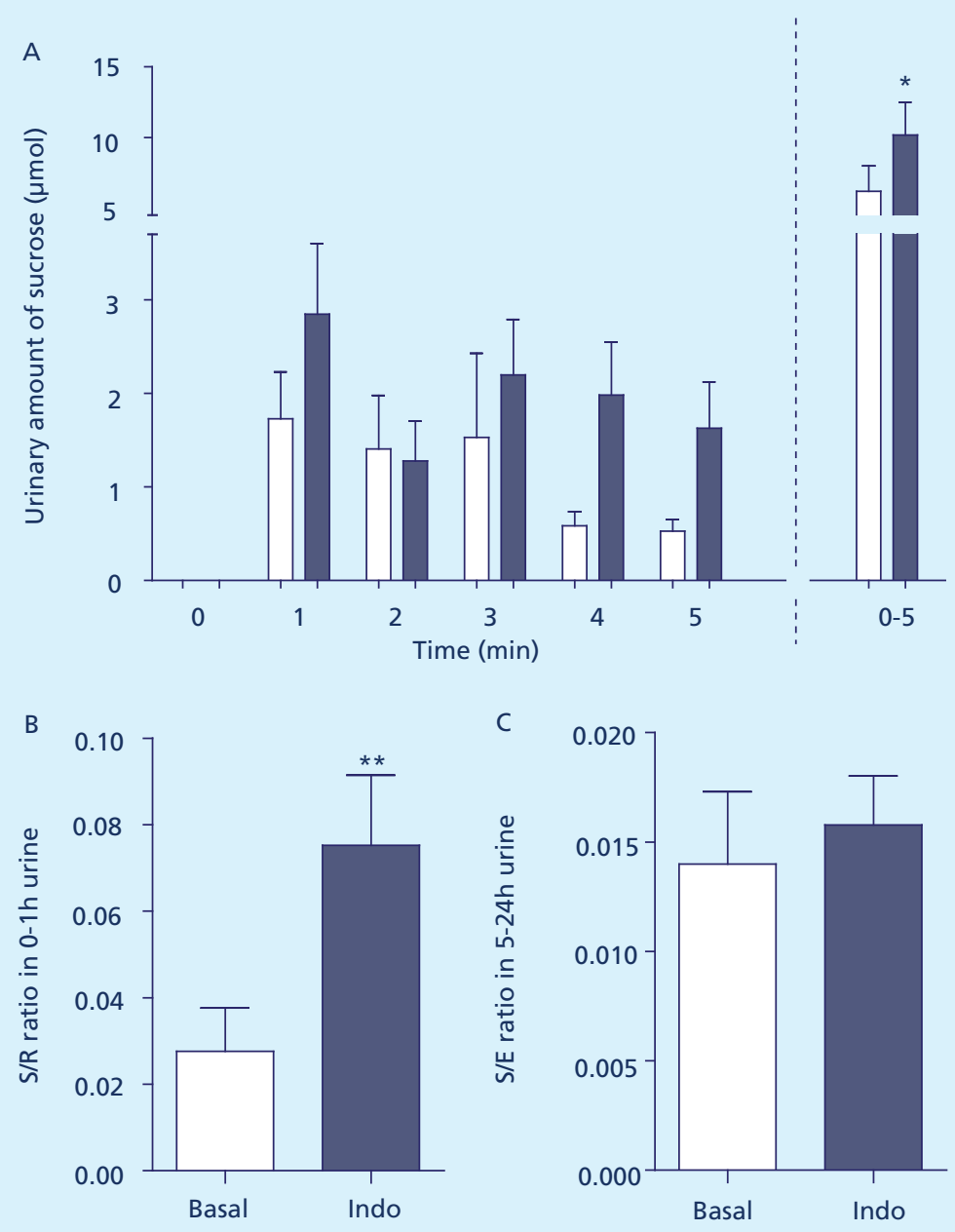

Basal

Indomethacin

Figure 4: Indomethacin increases gastroduodenal, but not colonic permeability

A) Indomethacin increases sucrose excretion in urine. B) Indomethacin elevated the sucrose/rhamnose (S/R) ratio in $0-1 \mathrm{~h}$ urine, reflecting enhanced gastroduodenal permeability. C) Indomethacin did not significantly increase large intestinal permeability, as shown by the $5-24 \mathrm{~h}$ urinary sucralose/erythritol (S/E) ratio. $\left({ }^{\star} P<.05,{ }^{\star \star} P<.01 \mathrm{com}-\right.$ pared to basal).

\section{Indomethacin-induced changes in gastroduodenal permeability}

Urinary excretion of sucrose is considered to reflect upper GI permeability $(25,32)$, and classically, high oral sucrose doses of $20 \mathrm{~g}(29)$ to $100 \mathrm{~g}(31,32)$ are used. Due to our sensitive detection method, we could reduce this oral dose to $1 \mathrm{~g}$. Analysis revealed significantly elevated $0-5 \mathrm{~h}$ urinary sucrose excretion $(9.9 \pm 2.4 \mu \mathrm{mol}$ versus $5.8 \pm 1.9 \mu \mathrm{mol} ; P<0.05$; Figure $4 A$ ). Plasma 
sucrose concentrations increased after intake of indomethacin compared to baseline $(1.8 \pm 0.2$ $\mu \mathrm{mol}$ versus $1.3 \pm 0.2 \mu \mathrm{mol} ; P<0.02)$. Interestingly, increased sucrose excretion was also observed in the last $3 \mathrm{~h}$ of urinary collection (Figure $4 A$ ), reflecting late intestinal sucrose uptake. Discrimination of increased gastroduodenal permeability in the indomethacin-challenged state was possible in the majority of individuals by early assessment of urinary sucrose excretion or plasma concentrations, however, accuracy could be improved (Table 2). In this study, sufficient discriminatory accuracy for indomethacin-induced increased gastroduodenal permeability was obtained using the $0-1$ h urinary sucrose/rhamnose ratio (Figure 4B; Table 2).

\section{Indomethacin-induced changes in large intestinal permeability}

The effect of indomethacin on large intestinal permeability was analyzed through determination of 5-24h sucralose excretion and the S/E ratio. In this study, no significantly increased sucralose excretion was observed after indomethacin $(52 \pm 13 \mu \mathrm{mol}$ versus baseline $35 \pm 7.6 \mu \mathrm{mol} ; P=0.20$ ). In line, indomethacin did not significantly change the $5-24 \mathrm{~h}$ urinary $\mathrm{S} / \mathrm{E}$ ratio $(P=0.10$; Figure $4 C)$. These results indicate that indomethacin did not significantly affect large intestinal permeability.

\section{DISCUSSION}

The novel method of detection enabled highly sensitive quantification of permeability sugar probes in urine and plasma. It allowed a 5-fold reduction in the classically applied dose of lactulose, necessary for small intestinal permeability analysis. Lactulose and rhamnose were complemented with three additional sugars permitting simultaneous assessment of gastroduodenal and colonic permeability. In the current study, the low-dose lactulose MS test was compared with the classical high-dose lactulose DS test in an established human model of indomethacininduced enhanced GI permeability in healthy individuals.

The noxious effect of NSAID such as indomethacin on the GI tract is evident (27), and small intestinal mucosal lesions have been described in healthy individuals after 2 weeks of diclofenac ingestion (21). In addition, Tibble et al. described intestinal inflammation measured by faecal calprotectin in $44 \%$ of patients taking NSAID (33), while Bjarnason et al. reported inflammation in up to $70 \%$ of long-term users (4). In the current study, we observed intestinal damage, reflected by enhanced plasma I-FABP levels, and inflammation measured by plasma calprotectin after only two doses of indomethacin (75 and $50 \mathrm{mg}$ p.o.). Our data indicate that even such short-term use of NSAID causes intestinal injury and disruption of the intestinal barrier in healthy individuals.

The MS test was designed to perform site-specific GI permeability analysis. Interestingly, increased gastroduodenal permeability following indomethacin could accurately be determined by sucrose in $1 \mathrm{~h}$ plasma, or by calculation of the $0-1 \mathrm{~h}$ urinary $\mathrm{S} / \mathrm{R}$ ratio. We consider that these early samples allow accurate gastroduodenal permeability assessment, since gastric emptying of 
liquid in fasted individuals takes approximately 40 minutes (22). Remarkably, we also observed high urinary sucrose excretion 3-5h after intake of the test mix in the indomethacin-challenged state, which may be explained by indomethacin-induced reductions in small intestinal sucrase activity as previously reported by Meddings et al. in rats (24).

Comparison of the MS and DS test showed that urinary $\mathrm{L} / \mathrm{R}$ ratio allowed assessment of indomethacin-induced permeability, especially in the first two hours after probe intake. For the MS test, individual sugar probe analysis revealed that increased urinary and plasma L/R ratio resulted from higher small intestinal permeability for disaccharides (lactulose) with unchanged permeability for monosaccharides (rhamnose). Remarkably, elevated disaccharide concentrations were observed to a lesser extent with the DS test, which also revealed decreased monosaccharide concentrations after indomethacin. Reduced monosaccharide excretion has been described previously in case of increased permeability $(17,26,34,35)$, and has been attributed to decreased mucosal absorptive surface due to intestinal disease such as celiac disease (5). However, this assumption can be negated based on the present study, since we observed increased monosaccharide levels when the low-dose MS test was used, performed in the same subjects. The latter data are consistent with data of Dawson et al. showing that monosaccharide permeation is increased in untreated celiac disease when corrected for mucosal surface area (10). Alternatively, the reduced monosaccharide excretion measured with the DS test may be caused by disturbance of the mucosal osmotic equilibrium by indomethacin in combination with the high lactulose dose. Intestinal permeation of monosaccharides is highly dependent on water absorption induced by agents or by oral hydration $(2,13,19)$. Indomethacin-induced disturbances in the countercurrent flow $(1,11)$ reduce the physiological hyperosmolarity of the villus tip (3). The combination of reduced villus osmolarity and the high luminal amount of lactulose may direct the water flux towards the lumen, and the resulting 'solvent drag' of monosaccharides could explain our observations with the DS test. The MS test appears to provide a more physiological situation for permeability analysis due to a reduction of the osmotic laxative lactulose.

Interestingly, the novel detection method enabled plasma-based permeability analysis. Plasma analysis provides several advantages. Frequent sampling is easy, and allows the assessment of permeability changes over time. Also, short-lived, more modest permeability changes, such as increased small intestinal permeability induced by physical exercise, are detected more easily $(36,37)$. Accurate plasma-based permeability analysis was possible using both tests within one hour after test mix intake. Importantly, these $1 \mathrm{~h} \mathrm{~L} / \mathrm{R}$ ratios strongly correlated with $\mathrm{L} / \mathrm{R}$ ratios in $0-1 \mathrm{~h}$ urine, and with plasma I-FABP levels determined at baseline. Finally, reduced monosaccharide rates measured with the DS test were more pronounced in urine than in plasma. We consider that these results may be explained by accumulation of sugar probes in the urinary bladder, thereby magnifying the effect.

The early increase in $\mathrm{L} / \mathrm{R}$ ratios and the high accuracy of early urinary samples compared to the $0-5 \mathrm{~h}$ urinary $\mathrm{L} / \mathrm{R}$ ratio are in line with the observations of Camilleri et al. who showed that 
$2 \mathrm{~h}$ after intake of a liquid test mix, approximately $50 \%$ of the mix was located in the colon ascendens (8). Taken together, these data suggest that earlier urinary collections than the classical $0-5 \mathrm{~h}$ collection are more useful for small intestinal permeability analysis.

Colonic inflammation and perforation have been described after NSAID intake (20), while only few studies report on the effect of indomethacin on large intestinal permeability $(18,24)$. We observed unaltered colon permeability after indomethacin, which is in line with results of Smecuol et al. who used $2 \mathrm{~g}$ of sucralose delivered by capsules (31). This led us to conclude that indomethacin does not lead to increased large intestinal permeability. Consequently, the usefulness of our MS test to detect increased colonic permeability remains to be determined in a situation with definite increased colon permeability such as in patients with colitis.

In conclusion, the current study shows that sensitive permeability analysis is feasible in plasma and urine using the MS or DS test. The MS test provides a more physiological situation for permeability analysis due to its reduced oral lactulose dose, and it allows accurate, site-specific information on gastroduodenal, small, and large intestinal permeability. Future studies are needed to assess the accuracy of the MS test in patient groups with increased intestinal permeability, before implementation of the MS test in standard clinical practice is possible. 


\section{REFERENCES}

1. Anthony A, Pounder RE, Dhillon AP, and Wakefield AJ. Vascular anatomy defines sites of indomethacin induced jejunal ulceration along the mesenteric margin. Gut 41: 763-770, 1997.

2. Bijlsma PB, Fihn BM, Sjoqvist A, Groot JA, Taminiau JA, and Jodal M. Water absorption enhances the uptake of mannitol and decreases Cr-EDTA/mannitol permeability ratios in cat small intestine in situ. Scand $\mathcal{F}$ Gastroenterol 37: 799-806, 2002.

3. Bijlsma PB, Peeters RA, Groot JA, Dekker PR, Taminiau JA, and Van Der Meer R. Differential in vivo and in vitro intestinal permeability to lactulose and mannitol in animals and humans: a hypothesis. Gastroenterology 108: 687-696, 1995.

4. Bjarnason I, Hayllar J, MacPherson AJ, and Russell AS. Side effects of nonsteroidal anti-inflammatory drugs on the small and large intestine in humans. Gastroenterology 104: 1832-1847, 1993.

5. Bjarnason I, MacPherson A, and Hollander D. Intestinal permeability: an overview. Gastroenterology 108: 15661581, 1995.

6. Bjarnason I, Peters TJ, and Veall N. 51Cr-EDTA test for intestinal permeability. Lancet 2: 523, 1984.

7. Bjarnason I, and Takeuchi K. Intestinal permeability in the pathogenesis of NSAID-induced enteropathy. $\mathcal{F}$ Gastroenterol 44 Suppl 19: 23-29, 2009.

8. Camilleri M, Nadeau A, Lamsam J, Nord SL, Ryks M, Burton D, Sweetser S, Zinsmeister AR, and Singh R. Understanding measurements of intestinal permeability in healthy humans with urine lactulose and mannitol excretion. Neurogastroenterol Motil 22: e15-26, 2010.

9. Dahlqvist A, and Borgstrom B. Digestion and absorption of disaccharides in man. Biochem J 81: 411-418, 1961.

10. Dawson DJ, Lobley RW, Burrows PC, Notman JA, Mahon M, and Holmes R. Changes in jejunal permeability and passive permeation of sugars in intestinal biopsies in coeliac disease and Crohn's disease. Clin Sci (Lond) 74: 427-431, 1988.

11. Dyess DL, Peeples GL, Ardell JL, Tacchi EJ, Roberts WS, Ferrara JJ, and Powell RW. Indomethacin-induced blood flow distribution in premature and full-term piglets. F Pediatr Surg 28: 1396-1400, 1993.

12. Farhadi A, Keshavarzian A, Holmes EW, Fields J, Zhang L, and Banan A. Gas chromatographic method for detection of urinary sucralose: application to the assessment of intestinal permeability. $\mathcal{F}$ Chromatogr B Analyt Technol Biomed Life Sci 784: 145-154, 2003.

13. Fihn BM, Sjoqvist A, and Jodal M. Permeability of the rat small intestinal epithelium along the villus-crypt axis: effects of glucose transport. Gastroenterology 119: 1029-1036, 2000.

14. Gotteland M, Cruchet S, and Verbeke S. Effect of Lactobacillus ingestion on the gastrointestinal mucosal barrier alterations induced by indometacin in humans. Alimentary pharmacology $\mathcal{E}$ therapeutics 15: 11-17, 2001.

15. Hiele M, Ghoos Y, Rutgeerts P, and Vantrappen G. Metabolism of erythritol in humans: comparison with glucose and lactitol. BrF Nutr 69: 169-176, 1993.

16. Holland J, Carey M, Hughes N, Sweeney K, Byrne PJ, Healy M, Ravi N, and Reynolds JV. Intraoperative splanchnic hypoperfusion, increased intestinal permeability, down-regulation of monocyte class II major histocompatibility complex expression, exaggerated acute phase response, and sepsis. Am f Surg 190: 393-400, 2005.

17. Jenkins AP, Menzies IS, Nukajam WS, and Creamer B. The effect of ingested lactulose on absorption of Lrhamnose, D-xylose, and 3-O-methyl-D-glucose in subjects with ileostomies. Scand f Gastroenterol 29: 820-825, 1994.

18. Jenkins AP, Trew DR, Crump BJ, Nukajam WS, Foley JA, Menzies IS, and Creamer B. Do non-steroidal antiinflammatory drugs increase colonic permeability? Gut 32: 66-69, 1991.

19. Krugliak P, Hollander D, Schlaepfer CC, Nguyen H, and Ma TY. Mechanisms and sites of mannitol permeability of small and large intestine in the rat. Dig Dis Sci 39: 796-801, 1994.

20. Laine L, Connors LG, Reicin A, Hawkey CJ, Burgos-Vargas R, SchnitzerTJ,Yu Q, and Bombardier C. Serious lower gastrointestinal clinical events with nonselective NSAID or coxib use. Gastroenterology 124: 288-292, 2003.

21. Maiden L, Thjodleifsson B, Theodors A, Gonzalez J, and Bjarnason I. A quantitative analysis of NSAID-induced small bowel pathology by capsule enteroscopy. Gastroenterology 128: 1172-1178, 2005.

22. Marciani L, Cox EF, Hoad CL, Pritchard S, Totman JJ, Foley S, Mistry A, Evans S, Gowland PA, and Spiller RC. Postprandial changes in small bowel water content in healthy subjects and patients with irritable bowel syndrome. Gastroenterology 138: 469-477, 477 e461, 2010.

23. Marshall JC, Christou NV, and Meakins JL. The gastrointestinal tract. The 'undrained abscess' of multiple organ failure. Ann Surg 218: 111-119, 1993.

24. Meddings JB, and Gibbons I. Discrimination of site-specific alterations in gastrointestinal permeability in the rat. Gastroenterology 114: 83-92, 1998.

25. Meddings JB, Sutherland LR, Byles NI, and Wallace JL. Sucrose: a novel permeability marker for gastroduodenal disease. Gastroenterology 104: 1619-1626, 1993. 
26. Menzies IS, Laker MF, Pounder R, Bull J, Heyer S, Wheeler PG, and Creamer B. Abnormal intestinal permeability to sugars in villous atrophy. Lancet 2: 1107-1109, 1979.

27. Morgan RW. Gastrointestinal toxicity of nonsteroidal antiinflammatory drugs. N Engl F Med 341: 1397; author reply 1398-1399, 1999.

28. Rombeau JL, and Takala J. Summary of round table conference: gut dysfunction in critical illness. Intensive Care Med 23: 476-479, 1997.

29. Sandek A, Bauditz J, Swidsinski A, Buhner S, Weber-Eibel J, von Haehling S, SchroedlW, Karhausen T, Doehner W, Rauchhaus M, Poole-Wilson P, Volk HD, Lochs H, and Anker SD. Altered intestinal function in patients with chronic heart failure. F Am Coll Cardiol 50: 1561-1569, 2007.

30. Sigthorsson G, Tibble J, Hayllar J, Menzies I, Macpherson A, Moots R, Scott D, Gumpel MJ, and Bjarnason I. Intestinal permeability and inflammation in patients on NSAIDs. Gut 43: 506-511, 1998.

31. Smecuol E, Bai JC, Sugai E, Vazquez H, Niveloni S, Pedreira S, Maurino E, and Meddings J. Acute gastrointestinal permeability responses to different non-steroidal anti-inflammatory drugs. Gut 49: 650-655, 2001.

32. Sutherland LR, Verhoef M, Wallace JL, Van Rosendaal G, Crutcher R, and Meddings JB. A simple, non-invasive marker of gastric damage: sucrose permeability. Lancet 343: 998-1000, 1994.

33. Tibble JA, Sigthorsson G, Foster R, Scott D, Fagerhol MK, Roseth A, and Bjarnason I. High prevalence of NSAID enteropathy as shown by a simple faecal test. Gut 45: 362-366, 1999.

34. Travis S, and Menzies I. Intestinal permeability: functional assessment and significance. Clin Sci (Lond) 82: 471-488, 1992.

35.van Nieuwenhoven MA, Geerling BJ, Deutz NE, Brouns F, and Brummer RJ. The sensitivity of the lactulose/rhamnose gut permeability test. Eur F Clin Invest 29: 160-165, 1999.

36. van Wijck K, Lenaerts K, van Loon LJ, Peters WH, Buurman WA, and Dejong CH. Exercise-induced splanchnic hypoperfusion results in gut dysfunction in healthy men. PLoS ONE 6: e22366, 2011.

37. Van Wijck K, Van Eijk HM, Buurman W, Dejong CH, and Lenaerts K. Novel analytical approach to a multisugar whole gut permeability assay. $\mathcal{F}$ Chromatogr B Analyt Technol Biomed Life Sci Minor revisions. 


\section{Supplementary figures}

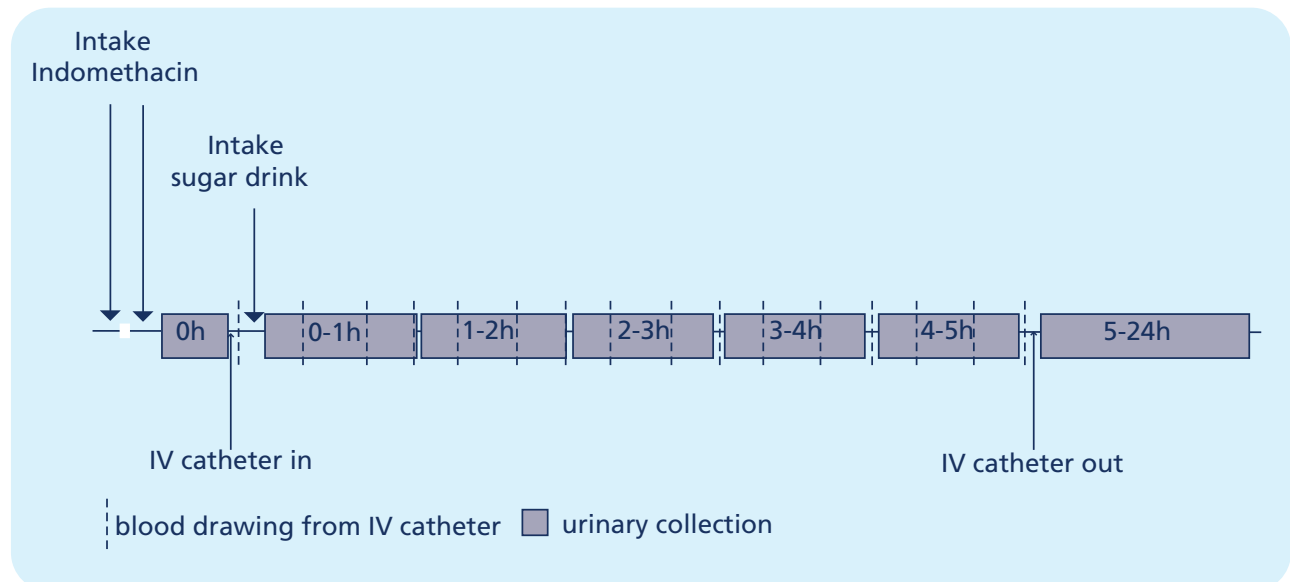

Supplementary figure 1: Study design of a test day

Overview of a test day, including moments of blood sample collection (indicated by dotted lines) and urinary collection (gray bars with annotation of sample collection times). Further indicated are the two moments of oral intake (i.e. the evening before the test day and the morning of the test day) of indomethacin or placebo pills, and the moment that the sugar test solution is ingested by the volunteers. 

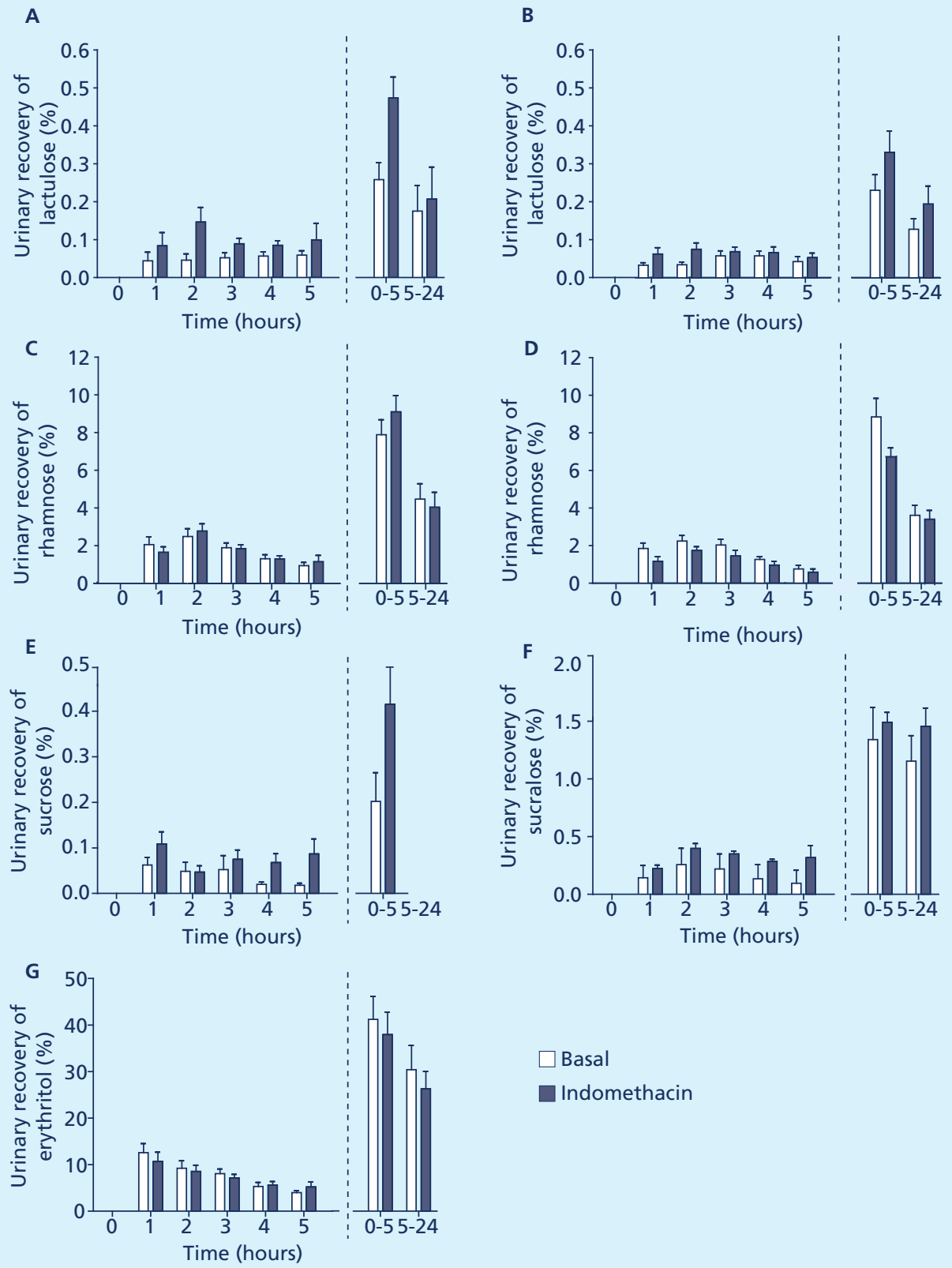

$\square$ Basal

Indomethacin

Supplementary figure 2: Urinary recovery of sugar probes after intake of indomethacin

Indomethacin-induced changes in urinary recovery of lactulose measured by the MS test (A) and DS test (B). Urinary recovery of rhamnose after intake of indomethacin measured by the MS test (C) and DS test (D). Indomethacin changed the urinary recovery of sucrose $(\mathrm{E})$, sucralose $(\mathbf{F})$, and erythritol $(\mathbf{G})$, all part of the MS test. 

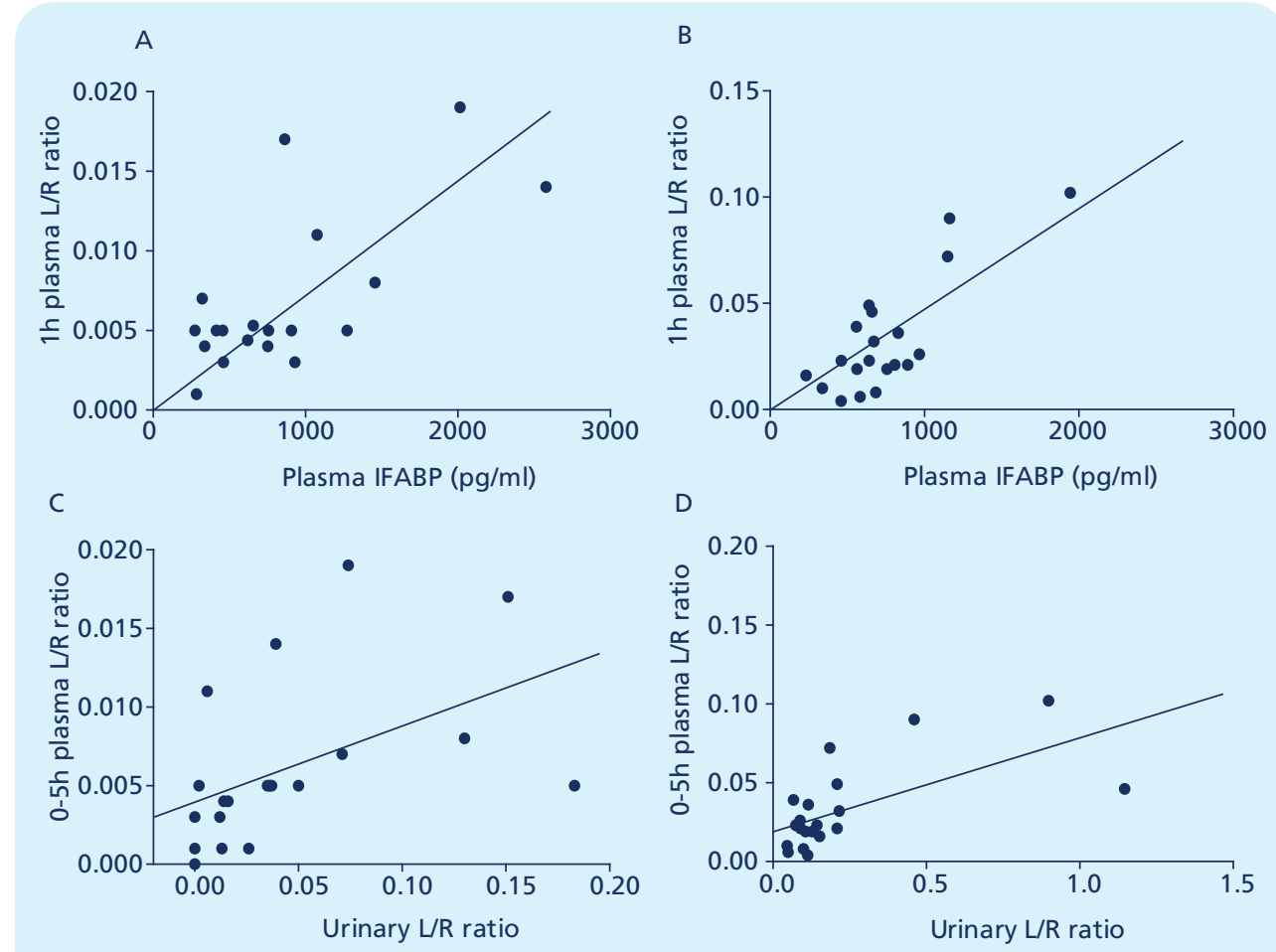

\section{Supplementary figure 3: Correlations}

Plasma I-FABP levels significantly correlate with $\mathrm{L} / \mathrm{R}$ ratios measured in $1 \mathrm{~h}$ plasma samples $\left(\mathrm{MS}\right.$ test: $\mathrm{R}_{\mathrm{S}}=0.53$; $P<.05$; (A) and DS test: $\mathrm{R}_{\mathrm{S}}=0.58 ; P<.01 ;(\mathrm{B})$ ). Plasma $0-5 \mathrm{~h} \mathrm{~L} / \mathrm{R}$ ratios correlate significantly with urinary $\mathrm{L} / \mathrm{R}$ ratios (MS test: $\mathrm{R}_{\mathrm{S}}=0.69 ; P<.001 ;(\mathrm{C})$ and $\mathrm{DS}$ test: $\left.\mathrm{R}_{\mathrm{S}}=0.59 ; P<.01 ;(\mathrm{D})\right)$. 


\section{ABSTRACT}

Splanchnic hypoperfusion is common in various pathophysiological conditions and often considered to lead to gut dysfunction. While it is known that physiological situations such as physical exercise also result in splanchnic hypoperfusion, the consequences of flow redistribution at the expense of abdominal organs remained to be determined. This study focuses on the effects of splanchnic hypoperfusion on the gut, and the relationship between hypoperfusion, intestinal injury and permeability during physical exercise in healthy men.

Healthy men cycled for 60 minutes at $70 \%$ of maximum workload. Splanchnic hypoperfusion was assessed using gastric tonometry. Blood, sampled every 10 minutes, was analyzed for enterocyte damage parameters (intestinal fatty acid binding protein (I-FABP) and ileal bile acid binding protein (I-BABP)). Changes in intestinal permeability were assessed using sugar probes. Furthermore, liver and renal parameters were assessed. Splanchnic perfusion rapidly decreased during exercise, reflected by increased gap ${ }_{\text {g-ap }} \mathrm{CO}_{2}$ from $-0.85 \pm 0.15$ to $0.85 \pm 0.42 \mathrm{kPa}(p<0.001)$. Hypoperfusion increased plasma I-FABP ( $615 \pm 118$ vs. $309 \pm 46 \mathrm{pg} / \mathrm{ml}, p<0.001)$ and I-BABP $(14.30 \pm 2.20$ vs. $5.06 \pm 1.27 \mathrm{ng} / \mathrm{ml}, p<0.001)$, and hypoperfusion correlated significantly with this small intestinal damage $\left(\mathrm{r}_{\mathrm{S}}=0.59 ; p<0.001\right)$. Last of all, plasma analysis revealed an increase in small intestinal permeability after exercise $(p<0.001)$, which correlated with intestinal injury $\left(r_{S}=0.50 ; p<0.001\right)$. Liver parameters, but not renal parameters were elevated.

In conclusion, exercise-induced splanchnic hypoperfusion results in quantifiable small intestinal injury. Importantly, the extent of intestinal injury correlates with transiently increased small intestinal permeability, indicating gut barrier dysfunction in healthy individuals. These physiological observations increase our knowledge of splanchnic hypoperfusion sequelae, and may help to understand and prevent these phenomena in patients. 


\section{INTRODUCTION}

Splanchnic hypoperfusion occurs in many pathophysiological conditions, and is often considered to lead to epithelial barrier dysfunction. Vascular disease, trauma, and shock can induce splanchnic hypoperfusion and ischemia $(13,40)$. In addition, gastrointestinal (GI) dysfunction is one of the more frequent complications in surgical patients, in whom organ perfusion and oxygen delivery are often impaired as a consequence of surgery-induced alterations in cardio-respiratory and metabolic demands (11). In critically ill patients, inadequate splanchnic blood flow causes intestinal damage, thereby compromising the intestinal mucosal barrier, potentially inducing and aggravating endotoxaemia and systemic inflammation (7).

Splanchnic hypoperfusion also occurs in physiological conditions. Young, healthy individuals endure episodes of splanchnic hypoperfusion during strenuous physical exercise (29), whereas older individuals may experience similar events during less exhausting activities. Moreover, there is considerable evidence supporting the theory that splanchnic hypoperfusion plays an important role in the development of GI complications in patients with chronic diseases, such as chronic heart failure or pulmonary disease $(17,20)$. Interestingly, the consequences of short-term splanchnic hypoperfusion on the organs in the splanchnic area remain to be determined.

In the current study, we focus on the direct effects of splanchnic hypoperfusion on gut, liver, and kidney epithelium in healthy young volunteers who perform moderate-to-high intensity physical exercise. This study is based on the fact that functional splanchnic hypoperfusion occurs during physical exercise, when rapid redistribution of the splanchnic blood flow occurs to secure supply of adequate amounts of oxygen and energy to the active muscle tissue, heart, and lungs $(29,35)$. In addition, during exercise a reduction of the total circulatory blood volume, caused by transpiration and inadequate fluid intake, can decrease cardiac output and compromise splanchnic perfusion even more (39). Especially during prolonged running or cycling, athletes can experience abdominal symptoms such as cramping, nausea, abdominal pain, and (bloody) diarrhea (33). This points towards compromised GI functioning, but only few studies have looked at exercise-induced intestinal mucosal lesions in man $(4,28)$.

In the current study, we determined the consequences of exercise-induced splanchnic hypoperfusion on gut, liver and kidney epithelium, with particular emphasis on intestinal barrier integrity loss in healthy individuals. 


\section{METHODS AND MATERIALS}

\section{Ethical approval}

This study was approved by the medical ethical committee of Maastricht University Medical Centre+, and conducted in accordance with the Declaration of Helsinki (revised version, October 2008, Seoul).

\section{Participant characteristics}

Fifteen healthy male volunteers were recruited via posters at the University. All volunteers spent 4 to 10 hours per week performing endurance sports as part of their normal life style. The volunteers had no abdominal complaints during daily activities, had not taken any medication for at least one month prior to participation, had no history of GI disease, and had had no abdominal surgery. Volunteers were informed about the nature and risks of the experiments. Written consent was obtained 5 days before the experiments.

Participants had a mean age of $23.6 \pm 0.7$ years and body mass index of $21.0 \pm 0.4 \mathrm{~kg} / \mathrm{m}^{2}$. Baseline characteristics and maximum workload capacity did not differ between the study groups. Subjects' characteristics are reported in Table 1. All participants were able to complete the 1-hour exercise bout, except for 1 volunteer, who discontinued cycling after 42 minutes due to exhaustion.

Table 1: Baseline characteristics of the healthy male participants ${ }^{1}$.

\begin{tabular}{|lcccc} 
& GI perfusion & $\begin{array}{c}\text { Intestinal } \\
\text { damage }\end{array}$ & GI permeability & $p$ value \\
\hline Group size (no. of participants) & 9 & 15 & 6 & \\
Age (years) & $24.2 \pm 1.0$ & $23.8 \pm 0.8$ & $25.0 \pm 0.6$ & 0.67 \\
Height (m) & $1.79 \pm 0.02$ & $1.80 \pm 0.02$ & $1.79 \pm 0.00$ & 0.79 \\
Weight (kg) & $68.4 \pm 2.8$ & $68.1 \pm 1.7$ & $66.8 \pm 3.8$ & 0.92 \\
Body Mass Index (kg/m ${ }^{2}$ ) & $20.3 \pm 0.6$ & $21.0 \pm 0.5$ & $20.8 \pm 0.7$ & 0.68 \\
Total body: fat percentage (\%) & $11.6 \pm 0.7$ & $11.9 \pm 0.8$ & $12.5 \pm 0.6$ & 0.81 \\
$\quad$ fat mass (kg) & $8.0 \pm 0.6$ & $8.1 \pm 0.6$ & $8.4 \pm 5.8$ & 0.92 \\
fat free mass (kg) & $61.1 \pm 2.6$ & $60.3 \pm 1.5$ & $59.4 \pm 3.8$ & 0.91 \\
Both legs: fat percentage (\%) & $12.6 \pm 0.8$ & $12.6 \pm 0.9$ & $13.5 \pm 0.6$ & 0.77 \\
$\quad$ fat mass (kg) & $3.1 \pm 0.3$ & $3.1 \pm 0.3$ & $3.3 \pm 0.3$ & 0.87 \\
lean mass (kg) & $20.2 \pm 1.2$ & $20.5 \pm 0.7$ & $20.1 \pm 1.5$ & 0.97 \\
\hline
\end{tabular}

Data are presented as mean \pm SEM. ${ }^{1}$ Of all study participants, three subjects participated in all three substudies, and four subjects participated in two substudies. Hence, in total, 20 individuals took part in the study. 


\section{Pre-exercise restrictions/arrangements}

Prior to the experiments, maximal workload capacity $\left(\mathrm{W}_{\max }\right)$ was assessed on a stationary cycle ergometer (Lode Excalibur, Groningen, the Netherlands), during electrocardiographic monitoring (MAC 5500, GE Medical Systems, Freiburg, Germany) to exclude cardiologic abnormalities. A dual energy X-ray absorptiometry scan (DXA ${ }^{\mathrm{TM}}$, Hologic Inc., the Netherlands) was performed to assess body composition.

Test subjects recorded dietary intake 2 days prior to the first experimental day, and maintained dietary intake as recorded for subsequent test days to prevent dietary influence. Participants were not allowed to consume alcohol or caffeine 2 days prior to each test day. Moreover, participants maintained normal activities of daily living, but refrained from strenuous physical activity. The evening before each test day, participants received a standardized meal (1.7 MJ, consisting of $62.6 \mathrm{~g}$ carbohydrate, $18.9 \mathrm{~g}$ protein and $7.9 \mathrm{~g}$ fat).

\section{Study design and sampling}

Subjects were tested after an overnight fast. The test design is depicted as Supplementary figure 1. In short, a catheter (20 Gauge, Braun, Melsungen, Germany) was placed in the participant's forearm vein. Every 10 minutes, blood was collected into pre-chilled EDTA tubes (Vacucontainer, Becton Dickinson (BD), Helsingborg, Sweden), and kept on ice. Urine was collected by the participants in 11 plastic cups before and within 1 hour after exercise. Urinary volume was recorded and $4 \mathrm{ml}$ was directly transferred to pre-chilled tubes. Blood and urine samples were centrifuged within 1 hour at $4^{\circ} \mathrm{C}$ at $2300 \mathrm{x}$ for 15 minutes and immediately stored at $-80^{\circ} \mathrm{C}$ until analysis. Following 1 hour rest in supine position, participants started cycling at $150 \mathrm{~W}$. After 3 minutes, workload was increased to $70 \%$ of the individual's pre-assessed $\mathrm{W}_{\max }$. Subjects maintained pedal rates of $60 \mathrm{rpm}$, and workload was decreased by $25 \mathrm{~W}$ if the participant was unable to maintain $60 \mathrm{rpm}$. Participants were only allowed to drink a maximum of $200 \mathrm{ml}$ tap water per 10 minutes. Faeces were collected by 6 participants, before and after cycling, and stored at $-4^{\circ} \mathrm{C}$ until analysis. Subjects were asked to report GI complaints experienced on the test day and the following day.

\section{Assessment of GI perfusion}

Gastric air tonometry was performed in 9 participants. The evening before and 1 hour prior to the experiment, participants ingested $150 \mathrm{mg}$ ranitidine orally (GlaxoSmithKline, Zeist, the Netherlands) to suppress gastric acid production, which interferes with carbon dioxide, thereby affecting tonometry results (16). A nasogastric Tonometrics catheter (8 French, Datex Ohmeda, Finland) was introduced and fixed to both nasal flares. Gastric $\mathrm{pCO}_{2}$ was measured at 10 -minute intervals using an automated capnograph (Tonocap TC-200, Datex Ohmeda, Finland). To avoid the use of an arterial catheter, an intravenous catheter (22 Gauge, BD) was placed in a dorsal hand vein and the hand was placed in a hot box heated to $60^{\circ} \mathrm{C}$, to arterialize the blood for 
measuring arterial $\mathrm{pCO}_{2}$ (22), using a Radiometer ABL 510 (Copenhagen, Denmark). Blood samples were collected simultaneously with the tonometry measurements and gastric-arterialized $\mathrm{pCO}_{2}\left(\mathrm{gap}_{\mathrm{g}-\mathrm{a}} \mathrm{pCO}_{2}\right)$ was calculated.

\section{Assessment of intestinal damage}

Intestinal fatty acid binding protein (I-FABP), a sensitive marker of intestinal cell damage, was used to determine small intestinal damage (9). Plasma I-FABP was determined using an enzyme linked-immunosorbent assay (ELISA) (Hycult Biotechnology, Uden, the Netherlands; detection window $20-5,000 \mathrm{pg} / \mathrm{ml}$ ) according to the manufacturer's instructions in samples taken before $(\mathrm{t}=0)$, during ( $\mathrm{t}=10-50$ minutes $)$ and after $(\mathrm{t}=60-120$ minutes $)$ cycling. Plasma ileal bile acid binding protein (I-BABP), a sensitive damage marker of ileal epithelium, was measured before $(\mathrm{t}=0)$ and after ( $\mathrm{t}=60$ minutes) cycling, using an in-house developed ELISA as previously described (detection window 0.32-5.0 ng/ml) (5).

All plasma parameters were measured in pre-exercise and post exercise plasma of all participants, resulting in a total number of 20 paired samples (without overlap due to subjects participating in more than 1 study), unless stated otherwise.

\section{Assessment of liver damage}

Liver injury was assessed by liver fatty acid binding protein (L-FABP), alanine transaminase (ALT), and aspartate transaminase (AST). Plasma L-FABP is a sensitive indicator of hepatocellular injury (45). However, as expression of L-FABP has been described in renal and intestinal epithelium (31), this marker was combined with the classically used parameters ALT and AST. In addition, we determined plasma levels of $\alpha$-glutathione S-transferase ( $\alpha$-GST), which is advocated to be an early and more specific marker of liver injury and unaffected by muscle injury or haemolysis $(36,47)$. All liver markers were determined before, immediately after, and 1 hour after cessation of cycling. Plasma L-FABP was measured using a commercial ELISA according to the manufacturer's instructions (Hycult, detection window $0.20-25 \mathrm{ng} / \mathrm{ml}$ ). Plasma ALT and AST were determined by routine enzymatic assays at the Clinical Chemistry Laboratory (CCL). Plasma $\alpha$-glutathione S-transferase ( $\alpha$-GST) levels were assessed using a sandwich ELISA, as described previously (24). 


\section{Assessment of kidney damage}

Urinary $\mathrm{N}$-acetyl-beta-(D)-glucosaminidase (NAG) was determined using an enzyme colorimetric assay according to the manufacturer's instructions (HaemoScan, Groningen, the Netherlands). Concentrations were normalized to urinary creatinine values, measured by routine assays at the CCL and expressed as Units/mmol creatinine.

\section{Assessment of GI permeability}

Gastrointestinal permeability was determined using a mix of $1 \mathrm{~g}$ lactulose (Centrafarm, EttenLeur, the Netherlands), $1 \mathrm{~g}$ sucralose (Brenntag, Sittard, the Netherlands), $1 \mathrm{~g}$ erythritol (Danisco, Copenhagen, Denmark), $1 \mathrm{~g}$ sucrose (Van Gilse, Dinteloord, the Netherlands), and 0.5 g L-rhamnose (Danisco) dissolved in $150 \mathrm{ml}$ tap water, in a subset of 6 participants.

The study design, depicted in the Supplementary data, consisted of 2 test days, one to test basal permeability and one for exercise-induced permeability. Participants fasted overnight. The sugar bolus was ingested after 30 minutes of rest in supine position, or after 30 minutes of cycling. Blood was sampled every 20 minutes and urine every hour, up to 5 hours post ingestion. All subjects urinated in plastic cups within 10 minutes of the instructed time point. In addition, subjects collected their 5-24 hour urine at home and returned the provided bottles the next morning, when urine was immediately processed.

Combined HPLC (Model PU-1980 pump, Jasco Benelux, Maarsen, the Netherlands) and mass spectrometry (Model LTQ-XL, Thermo Electron, Breda, the Netherlands) were used to determine urinary and plasma sugar concentrations. Lactulose and rhamnose, and the lactulose/L-rhamnose $(\mathrm{L} / \mathrm{R})$ ratio were determined in urinary samples to assess small intestinal permeability. Since physiological, transient permeability changes were expected, and our novel permeability assay allowed the detection of sugar probes in plasma, we also determined lactulose and rhamnose concentrations, and $\mathrm{L} / \mathrm{R}$ ratios in plasma. Both lactulose and rhamnose are degraded in the colon. Therefore, the ratio between two other, inert sugar probes, i.e. the sucralose/erythritol (S/E) ratio, was determined in the 5-24h urine to assess large intestinal permeability. Since we did not draw blood in this time period, we did not perform large intestinal permeability analysis in plasma.

\section{Assessment of inflammatory activation products and bacterial translocation}

Circulating myeloperoxidase (MPO) and calprotectin, antimicrobial proteins that are excreted from neutrophils upon activation, are considered early parameters of systemic inflammatory activation $(26,42)$. Active MPO is known to elicit secretion of other cytokines including tumor necrosis factor- $\alpha$ (TNF- $\alpha$ ) and interleukins (19), which were therefore less useful. MPO was measured in plasma before and after cycling using a commercial ELISA (Hycult; detection window $0.40-100 \mathrm{ng} / \mathrm{ml}$ ). Since evidence is accumulating that inflammatory products are produced in contracting and skeletal muscle $(23,30)$, we determined both circulating and faecal calpro- 
tectin. The latter is considered a strong indicator of intestinal inflammation in specific, and has been found to correlate to other parameters of intestinal inflammation in inflammatory bowel disease (43) and enteropathy induced by non-steroidal anti-inflammatory drugs (NSAID) (44). Calprotectin was measured before and after cycling, using a commercial calprotectin ELISA (standard 1.6-100 $\mathrm{ng} / \mathrm{ml}$ ). Faeces were collected by the participants in provided faecal sampling cups. Time of collection was reported by the participants. All faecal samples were collected within 7 hours after cycling. For faecal analysis, faeces were thawed, and $100 \mathrm{mg}$ was added to $4.9 \mathrm{ml}$ of extraction buffer (0.1 M Tris, $0.15 \mathrm{M} \mathrm{NaCl}, 1.0 \mathrm{M}$ urea, $10 \mathrm{mM} \mathrm{CaCl}_{2} 2 \mathrm{H}_{2} \mathrm{O}, 0.1 \mathrm{M}$ citric acid, $0.5 \%$ BSA, $\mathrm{pH} 8.0$ ) was added (46). After 30 minutes shaking, $1 \mathrm{ml}$ of suspension was centrifuged at $10,000 \mathrm{rpm}$ for 20 minutes at $4^{\circ} \mathrm{C}$. Faecal calprotectin concentrations were measured in supernatant using the calprotectin ELISA.

Endotoxin core antibodies in plasma were determined using EndoCab ELISA (Hycult Biotechnology, Uden, the Netherlands; detection window 0.13-8.0 IgG median units (GMU)/mL) according to the manufacturer's instructions in samples taken before and after cycling.

\section{Statistical analysis}

Statistical analysis was performed using GraphPad Prism (Version 5.00, GraphPad Software for Windows, San Diego California, USA). Normality of all data was verified by the KolmogorovSmirnov test. All normally distributed data are presented as mean \pm standard error of the mean (SEM), not normally distributed data as median and range. Outliers identified using Grubbs analysis were excluded from statistical analysis. Continuous data were analyzed using repeated measures analysis of variance with Tukey's or Dunnett's post-hoc test for multiple comparisons. Basal and post exercise values were compared using the non-parametric Wilcoxon signed rank test, considering the small number of study participants. Within-person correlations between gap $_{\mathrm{g}-\mathrm{a}} \mathrm{pCO}_{2}$ and plasma I-FABP, and plasma I-FABP and plasma L/R ratio were computed by normalizing both data sets, which enables the assessment of the pure association of both variables by calculating the Spearman correlation coefficient $\left(r_{S}\right)$. Linear regression was used to visualize the correlation. A $p<0.05$ was considered statistically significant. 


\section{RESULTS}

\section{Exercise-induced GI hypoperfusion}

Tonometry revealed a significant increase of gap $_{\mathrm{g}-\mathrm{a}} \mathrm{pCO}_{2}$ during exercise, from $-0.85 \pm 0.15 \mathrm{kPa}$ to $0.85 \pm 0.42 \mathrm{kPa}(p<0.001)$ after exercise, indicating functional splanchnic hypoperfusion (Figure 1A). The steepness of the gap ${ }_{\mathrm{g} \text {-ap }} \mathrm{CO}_{2}$ slope was most pronounced during the first 10 minutes of exercise, suggesting that functional splanchnic adaptations occur rapidly after altered perfusion demands in other parts of the body during physical activity. The gap $\mathrm{g}_{\mathrm{g}-\mathrm{a}} \mathrm{pCO}_{2}$ approximated baseline within 1 hour after exercise, reflecting fast recovery of splanchnic perfusion following a state of functional GI hypoperfusion. Interestingly, the recovery of GI perfusion was most prominent during the first 10 minutes post exercise, which is in line with the rapid adaptation of abdominal perfusion during the first 10 minutes of cycling.

\section{Exercise-induced small intestinal damage}

To assess hypoperfusion-induced effects on the small intestinal epithelium, plasma I-FABP levels were determined, which increased rapidly, from $309 \pm 46 \mathrm{pg} / \mathrm{ml}$ at baseline to $615 \pm 118 \mathrm{pg} / \mathrm{ml}$ $(p<0.001)$ after cycling (Figure $1 B$ ). Similar to the decreased gap $\mathrm{g}_{\mathrm{g}} \mathrm{pCO}_{2}$ levels (Figure $1 \mathrm{~A}$ ), circulating I-FABP levels declined substantially in the first 10 minutes post exercise, and gradually decreased further until baseline I-FABP level was reached approximately 50 minutes after cycling (Figure 1B). Interestingly, we did not observe a new peak in plasma I-FABP levels in the $60 \mathrm{~min}$ utes after cycling, suggesting that reperfusion injury did not occur in this time period.

The short half-life of I-FABP in plasma (approximately 11 minutes) allows to analyze the correlation of enterocyte damage with splanchnic hypoperfusion (45), calculating the withinperson correlation between gap $\mathrm{g}_{\mathrm{a}} \mathrm{pCO}_{2}$ and the change in circulating I-FABP. Because this correlation has not yet been described in healthy individuals, we determined the correlation between gap $_{\mathrm{g}-\mathrm{a}} \mathrm{pCO}_{2}$ and I-FABP 3 times, i.e. with a delay of 10, 20, and 30 minutes. Interestingly, I-FABP levels significantly correlated with gap $_{\mathrm{g}-\mathrm{a}} \mathrm{pCO}_{2}$ assessed at all measured time points $\left(\mathrm{r}_{\mathrm{S}}: 0.579(p<0.001) ; 0.592(p<0.001)\right.$ and $0.528(p<0.001)$, respectively). The correlation between gap g-ap $_{2} \mathrm{CO}_{2}$ and I-FABP determined in samples taken 20 minutes later, is depicted in Figure $1 C$.

Similar to I-FABP, cycling increased I-BABP levels $(5.06 \pm 1.27$ to $14.3 \pm 2.20 \mathrm{ng} / \mathrm{ml}(p<0.001$; Figure $3 \mathrm{~A}$ )), reflecting enterocyte damage in the ileum specifically. 


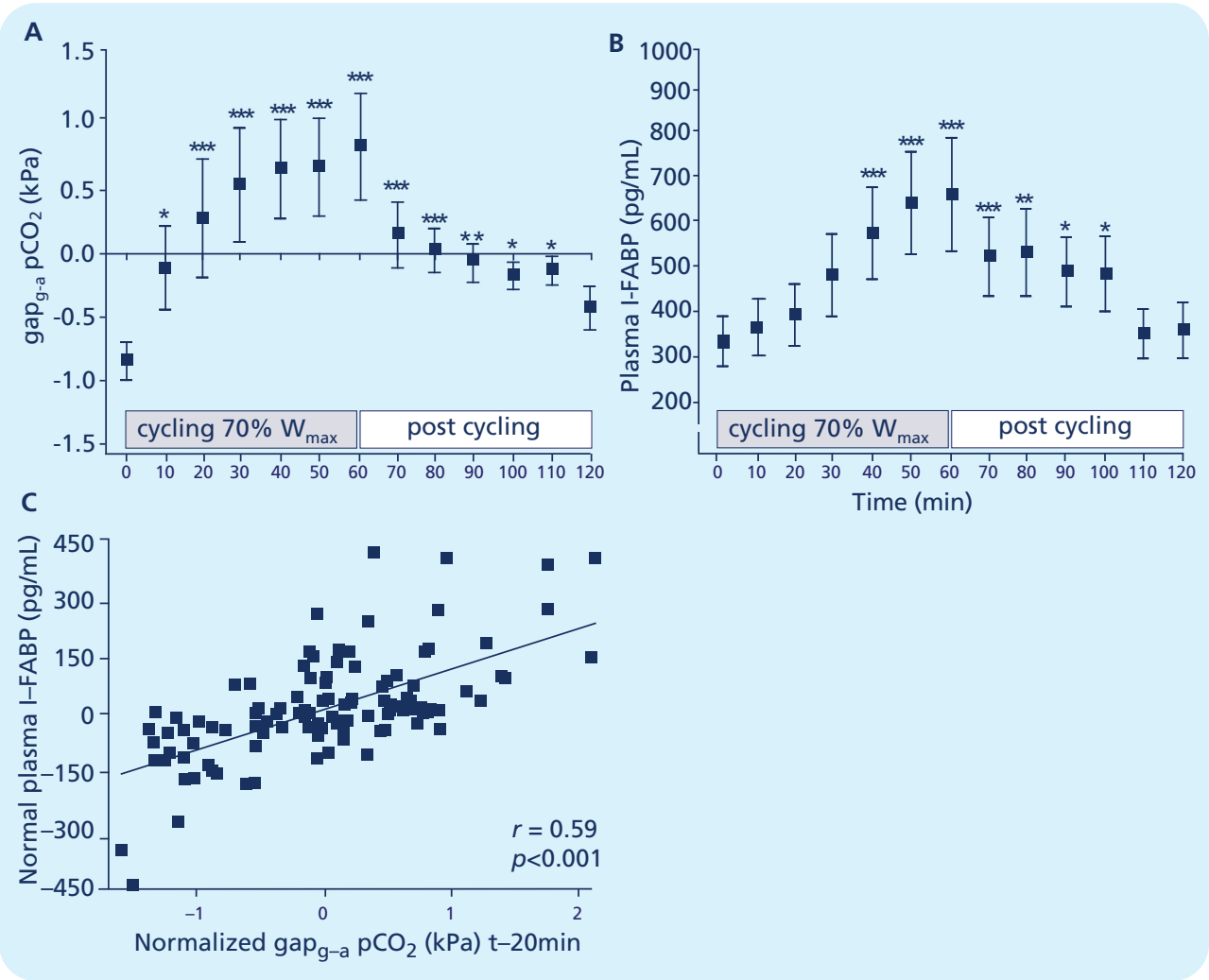

Figure 1: Physical exercise results in splanchnic hypoperfusion and intestinal cell damage.

A) Gastric tonometry shows decreased splanchnic perfusion during cycling and post cycling $(\mathrm{n}=9)$. B) Plasma I-FABP levels reflect the development of intestinal epithelial cell damage during cycling and post cycling in healthy volunteers $(n=20)$. C) Normalized plasma I-FABP levels measured during exercise tonometry correlate significantly with the normalized values of splanchnic hypoperfusion ( gap $_{\mathrm{g}-\mathrm{a}} \mathrm{pCO}_{2} \mathrm{t}-20 \mathrm{~min}$ ) in healthy men (samples from 9 men). Data are mean \pm SEM. Different from baseline $(\mathrm{t}=0)\left({ }^{\star} p<0.01,{ }^{\star \star} p<0.001,{ }^{\star \star \star} p<0.0001\right)$.

\section{Exercise-induced gastrointestinal complaints}

Two individuals reported nausea during the GI perfusion measurements with the nasogastric tube in situ, which resolved after the nasogastric tube was removed upon completion of the experiment. Three additional participants mentioned minor abdominal complaints during the test, including pain in epigastrio, flatulence and belching. The individuals reporting abdominal complaints did not show higher I-FABP levels during and post exercise than the individuals without abdominal complaints.

\section{Exercise-induced changes in liver parameters}

To obtain information on liver injury during exercise, we analyzed a set of four parameters: L-FABP, ALT, AST, and $\alpha$-GST. Plasma L-FABP levels significantly increased in all individuals upon exercise $(4.75 \pm 0.30$ to $14.2 \pm 1.38 \mathrm{ng} / \mathrm{ml}(p<0.001$; Figure $2 A)$ ). Plasma ALT and AST 
A

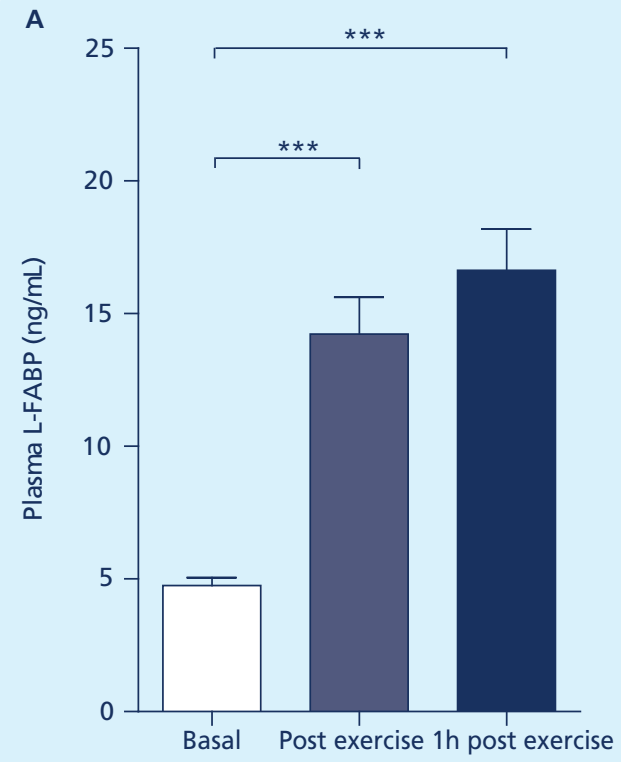

C

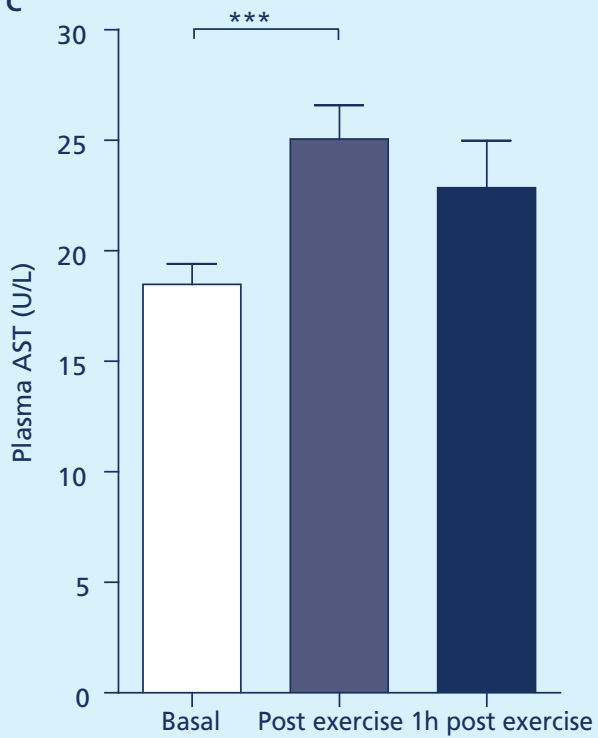

B

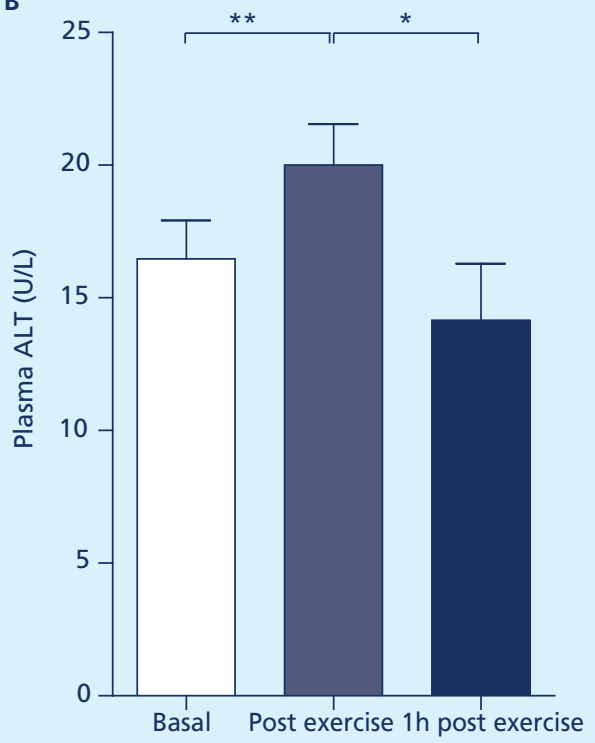

D

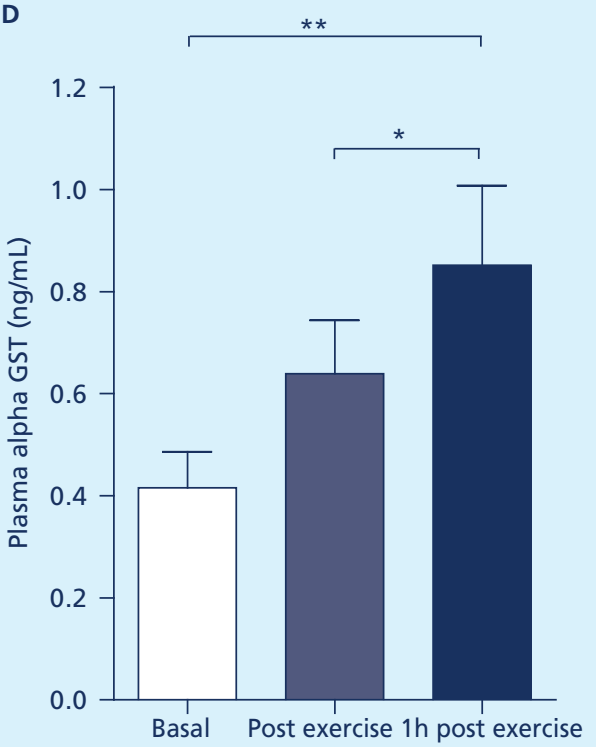

Figure 2: Physical exercise is associated with liver damage.

Plasma L-FABP (A), ALT (B), AST (C), and $\alpha$-GST (D) suggest liver injury after cycling. Data are mean \pm SEM $(n=20)$ of samples collected before, immediately after and 1 hour post cycling $\left({ }^{\star} p<0.05,{ }^{\star} p<<0.01\right.$, $\star \star \star p<0.001)$. 
levels increased from baseline $16.5 \pm 1.40$ to $20.0 \pm 1.60 \mathrm{U} / 1(p<0.01$; Figure $2 B)$ and $18.5 \pm 0.93$ to $23.8 \pm 0.98 \mathrm{U} / 1(p<0.001$; Figure $2 C)$, respectively. $\alpha$-GST levels also increased during and after exercise $(0.42 \pm 0.07$ to $0.64 \pm 0.10 \mathrm{ng} / \mathrm{ml}(p=0.12))$, reaching statistical significance at 1 hour post exercise $(0.85 \pm 0.16 \mathrm{ng} / \mathrm{ml}$ ( $p<0.01$ vs. baseline, $p<0.05$ vs. post exercise; Figure $2 D)$. Prolonged release of L-FABP and $\alpha-$ GST was observed at least for 1 hour post exercise, whereas plasma ALT and AST levels decreased 1 hour post exercise (Figure 2).

\section{Exercise-induced changes in renal parameters}

While we assumed that exercise caused renal damage, no significant changes in urinary NAG levels were observed ( $p>0.05$; Figure $3 B$ ). NAG, expressed in tubular epithelial cells (18), which are vulnerable to ischemic injury, is a sensitive marker of renal damage. Our data indicate that exercise, accompanied by enterocyte and hepatocellular injury, does not cause renal damage.

\section{Exercise-induced GI permeability changes}

To analyze whether the exercise-induced GI hypoperfusion and consequent enterocyte damage resulted in GI permeability changes, we analyzed small intestinal permeability, which was assessed as $L / R$ ratio in urine and plasma. All subjects were able to urinate within 10 minutes of the instructed time point, without using Foley catheters. Overall, a trend towards higher small intestinal permeability after exercise was observed in urine, which was especially pronounced in the first two hours of urinary collection (Figure $4 A$ ). However, no statistically significant changes were observed in the urinary $\mathrm{L} / \mathrm{R}$ ratio or the individual sugars after exercise. Since modest, transient changes in intestinal permeability only increase sugar probe excretion transiently, we considered that this increased probe excretion might be overshadowed by normal probe excretion, because all excreted probes accumulate in the bladder before leaving the body. Using our novel approach for sugar probe detection, we could assess the sugar probes in detail by analysis of plasma samples.

Plasma analysis revealed an overall increase in small intestinal permeability after exercise compared to rest $(p<0.001 ;$ Figure $4 B$ ). This increased plasma $\mathrm{L} / \mathrm{R}$ ratio was the result of significantly elevated lactulose concentrations after exercise ( $p<0.05$; data not shown), while plasma rhamnose levels remained unchanged. Interestingly, the plasma $L / R$ ratios correlated significantly with plasma I-FABP levels measured in the same samples during exercise $\left(\mathrm{r}_{\mathrm{s}}: 0.50, p<0.001\right.$; data not shown).

Next, correlations between the $0-1$ h urinary $\mathrm{L} / \mathrm{R}$ ratio and the $\mathrm{AUC}$ of the plasma $\mathrm{L} / \mathrm{R}$ ratios determined in the $0-1 \mathrm{~h}$ time period were evaluated for similarity, resulting in a $\mathrm{R}_{\mathrm{S}}$ of 0.42 $(p<0.05$; data not shown). Finally, the $\mathrm{S} / \mathrm{E}$ ratio was determined in the $5-24 \mathrm{~h}$ urinary collections to study large intestinal permeability changes. No changes in large intestinal permeability were observed (Figure 4C). 
A

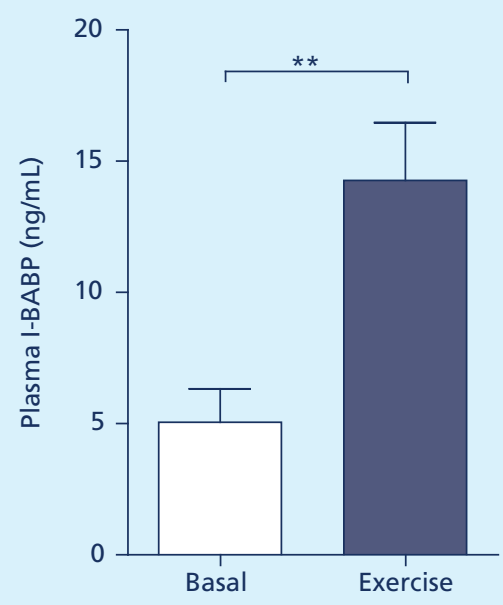

B

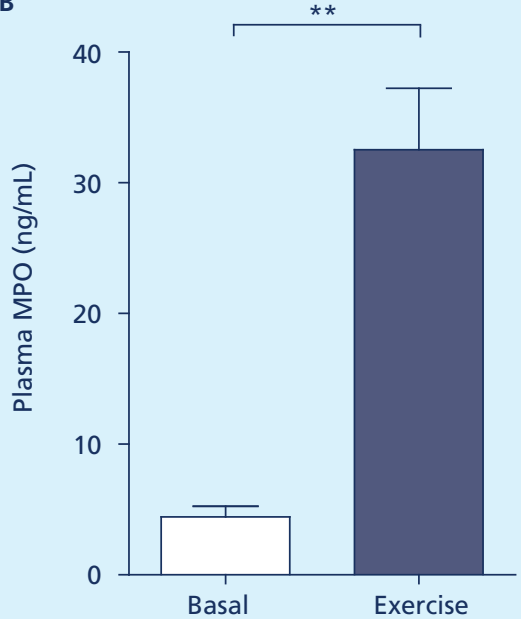

B

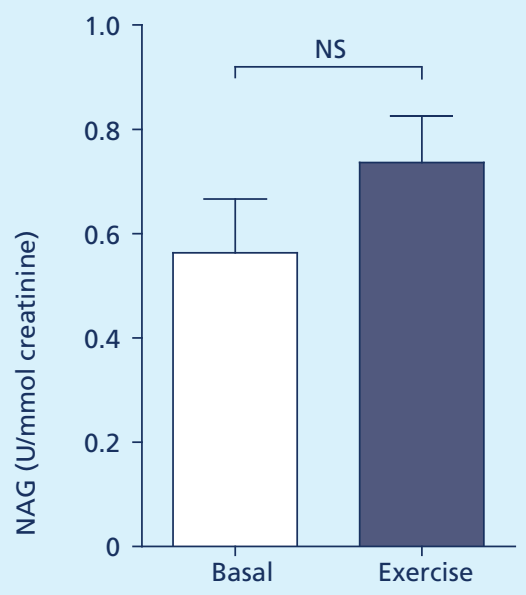

C

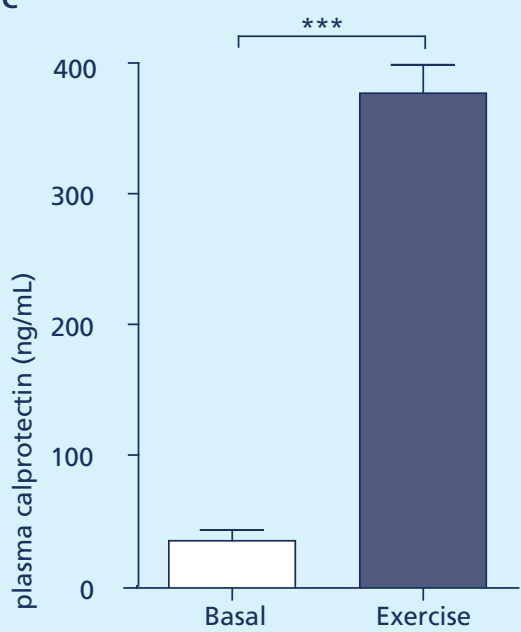

Figure 3: Physical exercise results in ileal epithelial damage and activates the acute phase response, without leading to renal injury.

A) Increased plasma IBABP levels point toward the development of exercise-induced ileal epithelial cell damage. B) Urinary NAG levels indicate absence of renal damage after cycling. B) Increased plasma MPO levels reveal an exercise-induced acute phase response in healthy volunteers. C) Increased plasma calprotectin levels indicate an exercise-induced acute phase response in healthy volunteers. Data are mean \pm SEM $(n=20)$. Different from baseline $(\mathrm{t}=0)\left(\mathrm{NS}\right.$, not significant; $\left.{ }^{\star \star} p<0.001,{ }^{\star \star \star} p<0.0001\right)$. 

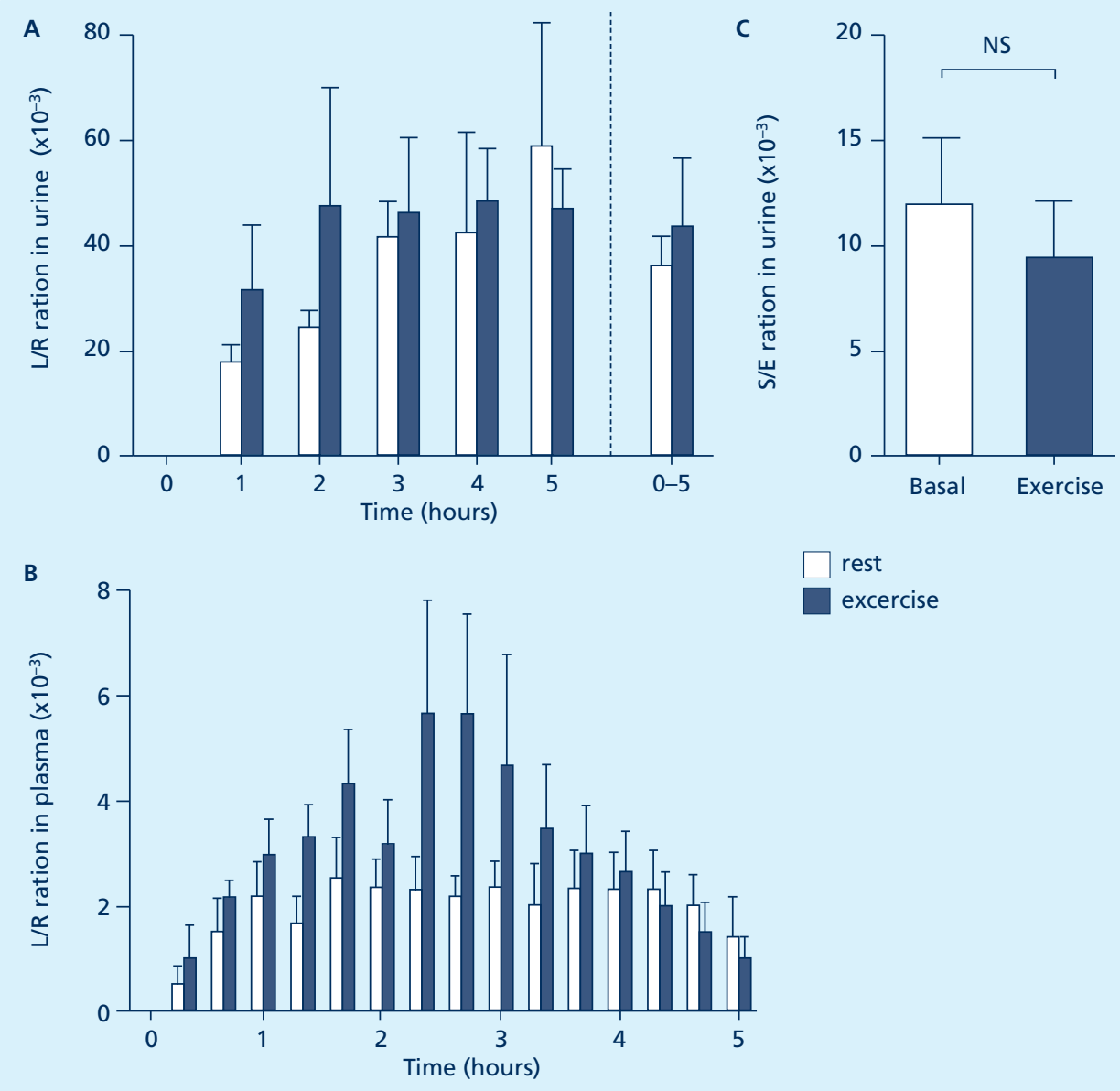

Figure 4: Physical exercise induces a mild increase in small intestinal permeability.

A) Urinary L/R ratios over time are elevated from baseline, especially in the first 2 hours after cycling, but not to a significant level. B) Plasma permeability analysis revealed an overall increase in small intestinal permeability after exercise compared to rest $(P<0.001)$. Plasma $\mathrm{L} / \mathrm{R}$ ratios after exercise at individual time points were not significantly different from L/R ratios in rest. C) Permeability of the large intestine, reflected by the 5-24h urinary S/E ratio, remains unaltered during exercise. Data are mean $\pm \operatorname{SEM}(n=6)$. Different from baseline $(t=0)(N S$, not significant; $\left.{ }^{\star \star} P<0.001\right)$.

\section{Exercise-induced inflammatory response without bacterial translocation}

Myeloperoxidase and calprotectin were analyzed as parameters of innate inflammation. Plasma MPO levels increased significantly in all individuals upon exercise, from baseline $4.4 \pm 0.8$ to $33 \pm 4.7 \mathrm{ng} / \mathrm{ml}$ after cycling $(p<0.001$; Figure $3 C$ ). In line with these results, a significant raise in circulating calprotectin levels was observed ( $p<0.0001$; Figure $3 D)$. Moreover, faecal calprotectin levels increased significantly upon exercise from baseline median $1.07 \mu \mathrm{g} / \mathrm{g}$ (range 0.49-2.20 $\mu \mathrm{g} / \mathrm{g}$ ) to $1.48 \mu \mathrm{g} / \mathrm{g}$ (range $0.59-40.82 \mu \mathrm{g} / \mathrm{g}, p<0.05$; data not shown). 
To assess the translocation of bacterial endotoxin in the circulation, EndoCab analysis was performed. The data did not reveal significant changes in endotoxin core antibody levels before and after cycling in the subgroup of participants that underwent permeability analysis (Supplementary figure $2 \mathrm{~A}$ ) or in the total group of participants (Supplementary figure $2 \mathrm{~B}$ ).

\section{DISCUSSION}

The redistribution of blood away from the splanchnic area is an adaptation of the body enabling perfusion of critical tissues in both physiological and pathophysiological situations. During physical exercise, reduction of the splanchnic circulation occurs to meet the increased demand for oxygen and substrates in the active muscle, thereby inducing a physiological state of hypoperfusion in the GI system (35). This study provides for the first time a comprehensive overview of the processes occurring in response to splanchnic hypoperfusion.

In this study, we demonstrate that in healthy individuals, one hour of physical exercise results in splanchnic hypoperfusion and small intestinal damage, reflected by an increase in plasma I-FABP. Interestingly, the extent of intestinal damage significantly correlated with the exerciseinduced hypoperfusion and with mild permeability changes in the small intestine. The most pronounced change in splanchnic perfusion, i.e. the most obvious increase in gap $\mathrm{g}_{\mathrm{g}-\mathrm{a}} \mathrm{pCO}_{2}$, occurred within the first 10 minutes of cycling, which implies that splanchnic perfusion rapidly decreases following the onset of exercise. The speed of adaptation of the splanchnic blood flow is further demonstrated by its fast recovery after cycling, which was also most prominent during the first 10 minutes following cessation of exercise. The rapid decline in splanchnic perfusion during cycling was followed by the development of significant intestinal damage with a time lag of 20 minutes. The data show a correlation between the exercise-induced splanchnic hypoperfusion and enterocyte damage (measured 20 minutes later), which is in line with animal studies and studies performed in critically ill patients demonstrating that intestinal hypoperfusion and ischemia result in intestinal cell damage (7).

The exercise-induced damage to the small intestine was measured using plasma I-FABP and I-BABP, both sensitive plasma parameters of enterocyte damage that are predominantly expressed in the upper half of the villi, where the mature enterocytes reside $(5,6,9)$. The susceptibility of the mature enterocyte population to low-flow states and ischemic events has been explained by the counter current exchange mechanism in the villus $(1,2)$, creating a constant low oxygen environment at the villus tip. Intestinal hypoperfusion deteriorates the oxygendeprived status of the villus tip even more, resulting in rapid loss of epithelial cell membrane integrity and concomitant I-FABP and I-BABP release. The increase in both I-FABP and I$\mathrm{BABP}$ indicates that exercise results in small intestinal damage in general, including the ileum. Interestingly, plasma I-FABP levels gradually decreased in the 60-minute 'reperfusion' period. 
The absence of a second peak in plasma I-FABP levels in this 60-minute period after splanchnic hypoperfusion indicates that detectable reperfusion injury did not occur in our healthy individuals. Our observations are in line with recent work on human intestinal ischemia/reperfusion (I/R) studies from our group showing that after 30 minutes of full intestinal ischemia in an isolated part of the jejunum during a Whipple procedure, plasma I-FABP levels rapidly increase, whereas during reperfusion, a gradual decrease in I-FABP levels was observed (8). During reperfusion, shedding of the damaged enterocytes prevented the jejunum from a massive I/R-induced inflammatory response (21). Taken together, these results reflect the ability of the gut to withstand episodes of partial or total deprivation of blood flow, with relatively little damage to the intestinal mucosa.

Having obtained strong indications for reduced splanchnic blood flow, we hypothesized that splanchnic outflow may be reduced during strenuous physical exercise, resulting in hepatocyte damage. Plasma levels of all assessed liver parameters (i.e. L-FABP, ALT, AST, and $\alpha-G S T$ ) elevated significantly after exercise. However, two other factors could be responsible for the phenomenon. First, the increase in these plasma parameters could theoretically be partly attributed to an exercise-induced reduction in plasma volume. The increases, however, largely exceeded the $10 \%$ decrease in plasma volume that has been described in athletes in more extreme settings than ours (15). Alternatively, muscle transaminase release is expected in response to (exerciseinduced) muscle injury $(27,34)$, while L-FABP release could be due to intestinal damage. Therefore, we additionally analyzed plasma $\alpha-G S T$, an early marker of hepatocyte injury unaffected by muscle injury, haemolysis, or intestinal injury $(36,47)$. In short, the significant rise in the assessed liver parameters after exercise strongly suggests the development of mild hepatocyte damage, which is in line with García-Pagàn et al. who reported a decrease in hepatic blood flow in patients with liver cirrhosis and portal hypertension after only 10 minutes of cycling at submaximal workload (10).

In contrast to the intestinal and liver data, we did not observe indications for renal damage during exercise. At this stage, it is unclear whether there was no hypoperfusion in the kidneys during cycling, or whether the kidney is more resistant to physiological hypoperfusion. Previous studies support the former, showing relatively unchanged renal flow after short-term splanchnic blood redistribution $(3,32)$.

In addition to the observed epithelial cell integrity loss, activation of the inflammatory response was observed. Inflammation is characterized by neutrophil influx and activation, resulting in the release of MPO and calprotectin, as seen in the present study. MPO plays a role in oxidative processes, while calprotectin and one of its constituents, myeloid-related protein- 8 (Mrp8, S100A8), are identified as strong activators of the innate Toll-like receptor 4 signaling pathway, resulting in the expression of pro-inflammatory cytokines (48). In addition to a rise in plasma calprotectin levels, increased faecal calprotectin concentrations were found after cycling in our healthy individuals, reflecting sequestration and activation of neutrophils in the intestinal 
mucosa, suggesting the gut to be an additional source of inflammatory products after physical exercise. Inflammation and neutrophil influx have been described previously in the human intestine, as part of ischemia/reperfusion injury (12), while a study by Mortensen et al., assessing femoral arterial-venous differences during knee extensor exercise, pointed towards release of calprotectin from muscle tissue during exercise (23). We consider the release of both calprotectin and MPO to be enhanced in sites of tissue injury and inflammation, including muscle tissue (during exercise), but also the gut mucosa. Loss of gut barrier function, due to cellular injury and inflammation, could result in bacterial translocation. Indeed, Jeukendrup et al. found mild endotoxaemia $(5-15 \mathrm{pg} / \mathrm{ml})$ and increased levels of C-reactive protein in the majority of participants after finishing an approximately $12 \mathrm{~h}$ long-distance triathlon (14). While our data did not point towards bacterial translocation, the existence of mucosal damage and inflammation in the GI tract did suggest loss of epithelial barrier function in our healthy volunteers. In line, analysis of the sugar probes in plasma using our novel HPLC/MS approach revealed increased small intestinal permeability, while these permeability changes correlated with the observed intestinal injury. Interestingly, the classical analysis of sugar probes in urine did not reveal significant changes in permeability, which might be explained by the mild and temporary nature of the increase in small intestinal permeability. In short, the absence of endotoxaemia and the mild changes in small intestinal permeability found in the current study underline the physiological basis of the exercise-induced changes in GI homeostasis.

In the current study, exercise did not significantly increase large intestinal permeability. It could be argued that the large intestine is better protected against episodes of hypoperfusion, or that blood flow in the large intestine is better maintained to prevent the translocation of bacteria and their products from the lumen to the circulation. The latter is suggested by a study of Murray et al. that found a decrease in rectal mucosal blood flow of almost $30 \%$ after acute physical stress (25), while decreases ranging from 43 to approximately $80 \%$ have been reported for splanchnic blood flow during physical exercise $(35,37)$.

In this study, we show the development of intestinal damage due to physiological hypoperfusion. Our data imply that the healthy human small intestine can withstand transient hypoperfusion, since the observed enterocyte injury did not produce gastrointestinal symptoms in our healthy individuals. However, prolonged episodes of hypoperfusion may cause more severe epithelial injury that may give rise to abdominal symptoms, as observed in endurance trained athletes (41). Furthermore, it is tempting to speculate that in individuals with hampered vascular function or decreased cardiac output, physiological conditions such as 'regular' physical exercise and other daily activities, will rapidly result in splanchnic hypoperfusion, ultimately leading to enterocyte damage and increased gut permeability. In patients with compromised vascular function, hypoperfusion-induced epithelial dysfunction may give rise to endotoxaemia, thereby enhancing their state of chronic inflammation, as suggested by the group of Anker for patients with chronic heart failure $(17,38)$. 
We assume that in addition to such patients, also relatively healthy elderly experience episodes of splanchnic hypoperfusion during daily activities due to the ageing of the splanchnic vascular system.

In conclusion, our study demonstrates that a single, 60-minute bout of moderate-to-high intensity exercise is accompanied by splanchnic hypoperfusion and transient epithelial integrity loss in healthy young males, reflecting the ability of the gut to withstand to episodes of physiological stress with minor consequences. 


\section{REFERENCES}

1. Blikslager AT. Life in the gut without oxygen: adaptive mechanisms and inflammatory bowel disease. Gastroenterology 134: 346-348, 2008.

2. Blikslager AT MA, Gookin JL, Jones SL, Odle J. Restoration of barrier function in injured intestinal mucosa. Physiol Review 87 545-564, 2007.

3. Brugger LE, Beldi G, Beck M, Porta F, Bracht H, Candinas D, Takala J, and Jakob SM. Splanchnic vasoregulation after major abdominal surgery in pigs. World journal of surgery 34: 2057-2063, 2010.

4. Choi SC, Choi SJ, Kim JA, Kim TH, Nah YH, Yazaki E, and Evans DF. The role of gastrointestinal endoscopy in long-distance runners with gastrointestinal symptoms. European journal of gastroenterology $\mathcal{E}$ hepatology 13: 1089-1094, 2001.

5. Derikx JP, Blijlevens NM, Donnelly JP, Fujii H, Kanda T, van Bijnen AA, Heineman E, and Buurman WA. Loss of enterocyte mass is accompanied by diminished turnover of enterocytes after myeloablative therapy in haematopoietic stem-cell transplant recipients. Ann Oncol 20: 337-342, 2009.

6. Derikx JP, Matthijsen RA, de Bruine AP, van Bijnen AA, Heineman E, van Dam RM, Dejong CH, and Buurman WA. Rapid reversal of human intestinal ischemia-reperfusion induced damage by shedding of injured enterocytes and reepithelialisation. PloS ONE 3: e3428, 2008.

7. Derikx JP, Poeze M, van Bijnen AA, Buurman WA, and Heineman E. Evidence for intestinal and liver epithelial cell injury in the early phase of sepsis. Shock 28: 544-548, 2007.

8. Derikx JP, van Waardenburg DA, Thuijls G, Willigers HM, Koenraads M, van Bijnen AA, Heineman E, Poeze M, Ambergen T, van Ooij A, van Rhijn LW, and Buurman WA. New Insight in Loss of Gut Barrier during Major Non-Abdominal Surgery. PloS ONE 3: e3954, 2008.

9. Derikx JP, Vreugdenhil AC, Van den Neucker AM, Grootjans J, van Bijnen AA, Damoiseaux JG, van Heurn LW, Heineman E, and Buurman WA. A pilot study on the noninvasive evaluation of intestinal damage in celiac disease using I-FABP and L-FABP. Fournal of clinical gastroenterology 43: 727-733, 2009.

10. Garcia-Pagan JC, Santos C, Barbera JA, Luca A, Roca J, Rodriguez-Roisin R, Bosch J, and Rodes J. Physical exercise increases portal pressure in patients with cirrhosis and portal hypertension. Gastroenterology 111: 13001306, 1996.

11. Giglio MT, Marucci M, Testini M, and Brienza N. Goal-directed haemodynamic therapy and gastrointestinal complications in major surgery: a meta-analysis of randomized controlled trials. Br F Anaesth 103: 637-646, 2009 .

12. Grootjans J, Lenaerts K, Derikx JP, Matthijsen RA, de Bruine AP, van Bijnen AA, van Dam RM, Dejong CH, and Buurman WA. Human intestinal ischemia-reperfusion-induced inflammation characterized: experiences from a new translational model. The American journal of pathology 176: 2283-2291, 2010.

13. Holland J, Carey M, Hughes N, Sweeney K, Byrne PJ, Healy M, Ravi N, and Reynolds JV. Intraoperative splanchnic hypoperfusion, increased intestinal permeability, down-regulation of monocyte class II major histocompatibility complex expression, exaggerated acute phase response, and sepsis. American journal of surgery 190: 393-400, 2005.

14. Jeukendrup AE, Vet-Joop K, Sturk A, Stegen JH, Senden J, Saris WH, and Wagenmakers AJ. Relationship between gastro-intestinal complaints and endotoxaemia, cytokine release and the acute-phase reaction during and after a long-distance triathlon in highly trained men. Clin Sci (Lond) 98: 47-55, 2000.

15. Kavouras SA. Assessing hydration status. Current opinion in clinical nutrition and metabolic care 5: 519-524, 2002.

16. Kolkman JJ, Groeneveld AB, and Meuwissen SG. Effect of ranitidine on basal and bicarbonate enhanced intragastric PCO2: a tonometric study. Gut 35: 737-741, 1994.

17. Krack A, Richartz BM, Gastmann A, Greim K, Lotze U, Anker SD, and Figulla HR. Studies on intragastric $\mathrm{PCO}_{2}$ at rest and during exercise as a marker of intestinal perfusion in patients with chronic heart failure. Eur f Heart Fail 6: 403-407, 2004.

18. Liangos O, Perianayagam MC, Vaidya VS, Han WK, Wald R, Tighiouart H, MacKinnon RW, Li L, Balakrishnan VS, Pereira BJ, Bonventre JV, and Jaber BL. Urinary N-acetyl-beta-(D)-glucosaminidase activity and kidney injury molecule-1 level are associated with adverse outcomes in acute renal failure. $\mathcal{F}$ Am Soc Nephrol 18: 904912, 2007.

19. Malle E, Buch T, and Grone HJ. Myeloperoxidase in kidney disease. Kidney Int 64: 1956-1967, 2003.

20. Mangi AA, Christison-Lagay ER, Torchiana DF, Warshaw AL, and Berger DL. Gastrointestinal complications in patients undergoing heart operation: an analysis of 8709 consecutive cardiac surgical patients. Annals of surgery 241: 895-901; discussion 901-894, 2005.

21. Matthijsen RA, Derikx JP, Kuipers D, van Dam RM, Dejong CH, and Buurman WA. Enterocyte shedding and epithelial lining repair following ischemia of the human small intestine attenuate inflammation. PLoS ONE [Electronic Resource] 4: e7045, 2009. 
22. McLoughlin P, Popham P, Linton RA, Bruce RC, and Band DM. Use of arterialized venous blood sampling during incremental exercise tests. F Appl Physiol 73: 937-940, 1992.

23. Mortensen OH, Andersen K, Fischer C, Nielsen AR, Nielsen S, Akerstrom T, Aastrom MB, Borup R, and Pedersen BK. Calprotectin is released from human skeletal muscle tissue during exercise. The fournal of physiology 586: 3551-3562, 2008.

24. Mulder TP, Peters WH, Court DA, and Jansen JB. Sandwich ELISA for glutathione S-transferase $\alpha-1-1$ : plasma concentrations in controls and in patients with gastrointestinal disorders. Clin Chem 42: 416-419, 1996.

25. Murray CD, Flynn J, Ratcliffe L, Jacyna MR, Kamm MA, and Emmanuel AV. Effect of acute physical and psychological stress on gut autonomic innervation in irritable bowel syndrome. Gastroenterology 127: 1695-1703, 2004 .

26. Nakajima T, Kurano M, Hasegawa T, Takano H, Iida H, Yasuda T, Fukuda T, Madarame H, Uno K, Meguro K, Shiga T, Sagara M, Nagata T, Maemura K, Hirata Y, Yamasoba T, and Nagai R. Pentraxin3 and high-sensitive C-reactive protein are independent inflammatory markers released during high-intensity exercise. European journal of applied physiology 110: 905-913, 2010.

27. Nathwani RA, Pais S, Reynolds TB, and Kaplowitz N. Serum alanine aminotransferase in skeletal muscle diseases. Hepatology (Baltimore, Md 41: 380-382, 2005.

28. Oktedalen O, Lunde OC, Opstad PK, Aabakken L, and Kvernebo K. Changes in the gastrointestinal mucosa after long-distance running. Scand J Gastroenterol 27: 270-274, 1992.

29. Otte JA, Oostveen E, Geelkerken RH, Groeneveld AB, and Kolkman JJ. Exercise induces gastric ischemia in healthy volunteers: a tonometry study. F Appl Physiol 91: 866-871, 2001.

30. Pedersen BK, and Febbraio MA. Muscle as an endocrine organ: focus on muscle-derived interleukin-6. Physiological reviews 88: 1379-1406, 2008.

31. Pelsers MM, Hermens WT, and Glatz JF. Fatty acid-binding proteins as plasma markers of tissue injury. Clinica chimica acta; international journal of clinical chemistry 352: 15-35, 2005.

32. Perko MJ, Nielsen HB, Skak C, Clemmesen JO, Schroeder TV, and Secher NH. Mesenteric, coeliac and splanchnic blood flow in humans during exercise. The fournal of physiology 513 ( Pt 3): 907-913, 1998.

33. Peters HP, Bos M, Seebregts L, Akkermans LM, van Berge Henegouwen GP, Bol E, Mosterd WL, and de Vries WR. Gastrointestinal symptoms in long-distance runners, cyclists, and triathletes: prevalence, medication, and etiology. The American journal of gastroenterology 94: 1570-1581, 1999.

34. Pratt DS, and Kaplan MM. Evaluation of abnormal liver-enzyme results in asymptomatic patients. The New England journal of medicine 342: 1266-1271, 2000.

35. Qamar MI, and Read AE. Effects of exercise on mesenteric blood flow in man. Gut 28: 583-587, 1987.

36. Rees GW, Trull AK, and Doyle S. Evaluation of an enzyme-immunometric assay for serum alpha-glutathione S-transferase. Ann Clin Biochem 32 ( Pt 6): 575-583, 1995.

37. Rowell LB. Regulation of splanchnic blood flow in man. Physiologist 16: 127-142, 1973.

38. Sandek A, Bauditz J, Swidsinski A, Buhner S, Weber-Eibel J, von Haehling S, Schroedl W, Karhausen T, Doehner W, Rauchhaus M, Poole-Wilson P, Volk HD, Lochs H, and Anker SD. Altered intestinal function in patients with chronic heart failure. $\mathcal{F}$ Am Coll Cardiol 50: 1561-1569, 2007.

39. Sawka MN, Young AJ, Francesconi RP, Muza SR, and Pandolf KB. Thermoregulatory and blood responses during exercise at graded hypohydration levels. F Appl Physiol 59: 1394-1401, 1985.

40. Sreenarasimhaiah J. Diagnosis and management of intestinal ischaemic disorders. BMJ 326: 1372-1376, 2003.

41. ter Steege RW, Van der Palen J, and Kolkman JJ. Prevalence of gastrointestinal complaints in runners competing in a long-distance run: an internet-based observational study in 1281 subjects. Scand $\mathcal{F}$ Gastroenterol 43: 14771482, 2008.

42. Thuijls G, Derikx JP, Prakken FJ, Huisman B, van Bijnen Ing AA, van Heurn EL, Buurman WA, and Heineman E. A pilot study on potential new plasma markers for diagnosis of acute appendicitis. The American journal of emergency medicine 29: 256-260, 2011.

43. Tibble J, Teahon K, Thjodleifsson B, Roseth A, Sigthorsson G, Bridger S, Foster R, Sherwood R, Fagerhol M, and Bjarnason I. A simple method for assessing intestinal inflammation in Crohn's disease. Gut 47: 506-513, 2000 .

44. Tibble JA, Sigthorsson G, Foster R, Scott D, Fagerhol MK, Roseth A, and Bjarnason I. High prevalence of NSAID enteropathy as shown by a simple faecal test. Gut 45: 362-366, 1999.

45. van de Poll MC, Derikx JP, Buurman WA, Peters WH, Roelofs HM, Wigmore SJ, and Dejong CH. Liver manipulation causes hepatocyte injury and precedes systemic inflammation in patients undergoing liver resection. World journal of surgery 31: 2033-2038, 2007.

46. van der Sluijs Veer G, van den Hoven B, Russel MG, and van den Bergh FA. Time-resolved fluorimetric immunoassay of calprotectin: technical and clinical aspects in diagnosis of inflammatory bowel diseases. Clin Chem Lab Med 44: 292-298, 2006. 
47. van Griensven JM, Burggraaf KJ, Gerloff J, Gunzler WA, Beier H, Kroon R, Huisman LG, Schoemaker RC, Kluft K, and Cohen AF. Effects of changing liver blood flow by exercise and food on kinetics and dynamics of saruplase. Clin Pharmacol Ther 57: 381-389, 1995.

48. Vogl T, Tenbrock K, Ludwig S, Leukert N, Ehrhardt C, van Zoelen MA, Nacken W, Foell D, van der Poll T, Sorg C, and Roth J. Mrp8 and Mrp14 are endogenous activators of Toll-like receptor 4, promoting lethal, endotoxin-induced shock. Nat Med 13: 1042-1049, 2007. 


\section{Supplementary figure 1}
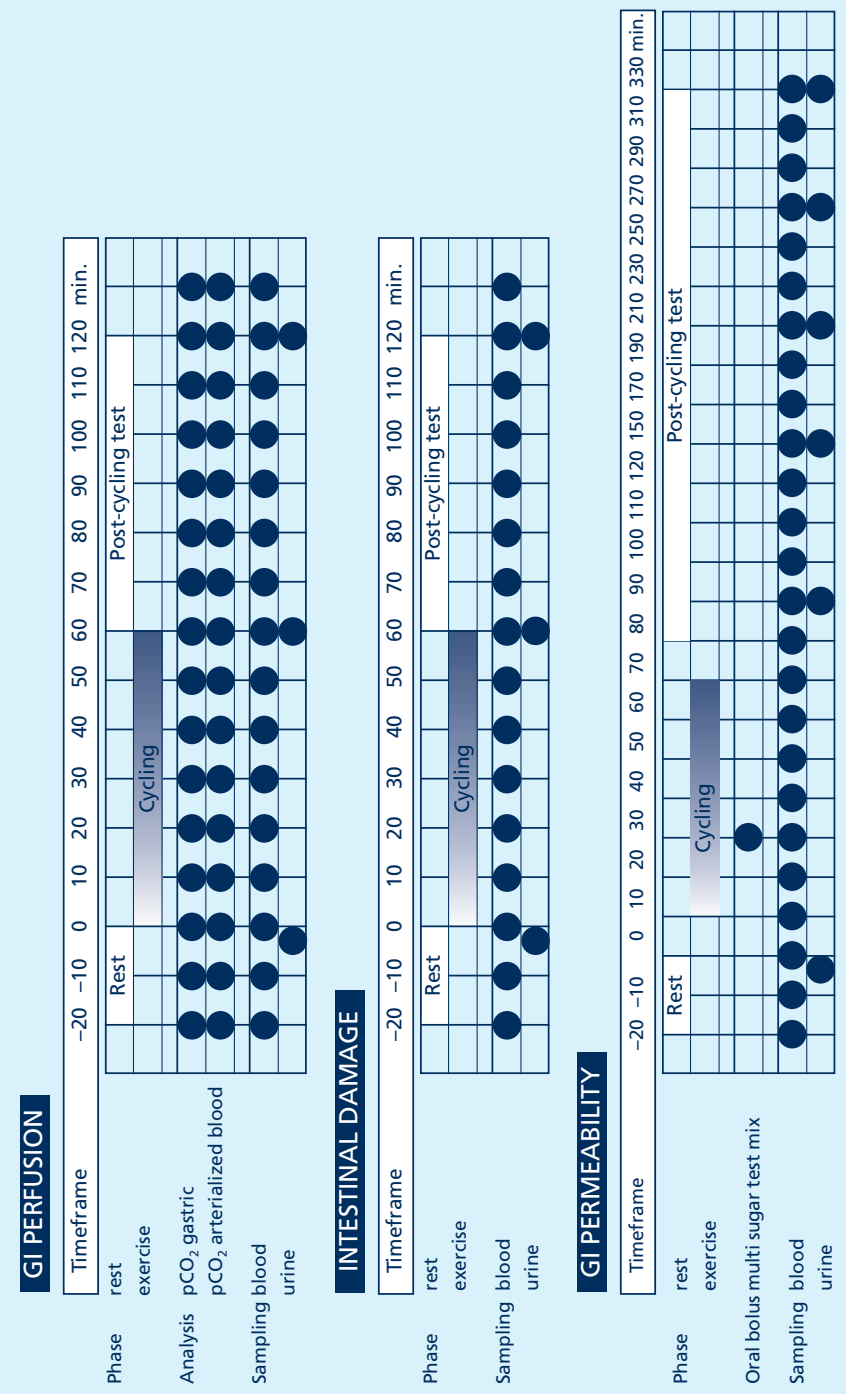
Supplementary figure 2

A

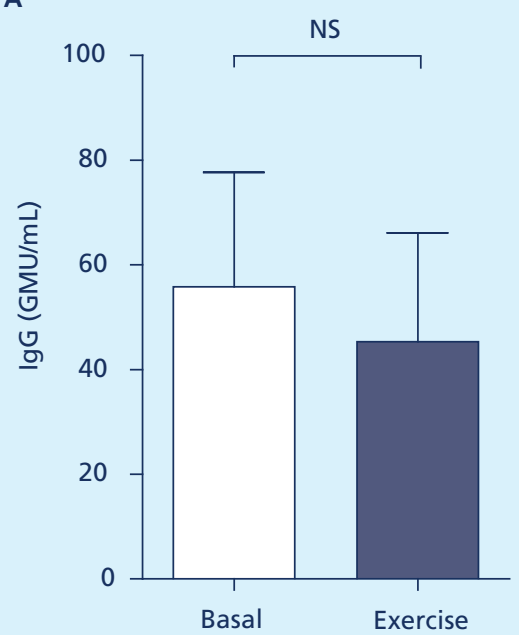

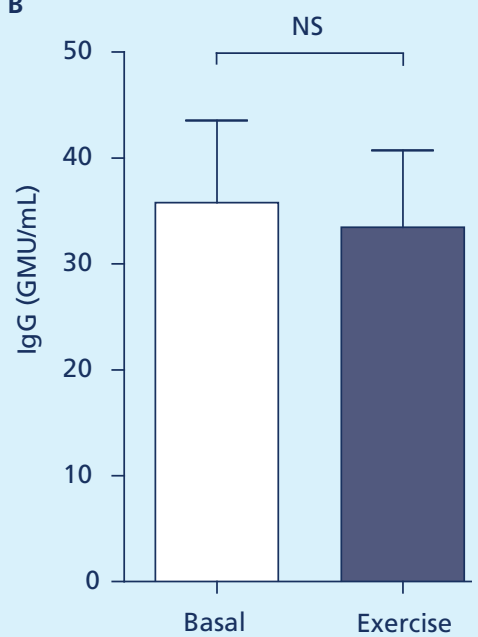




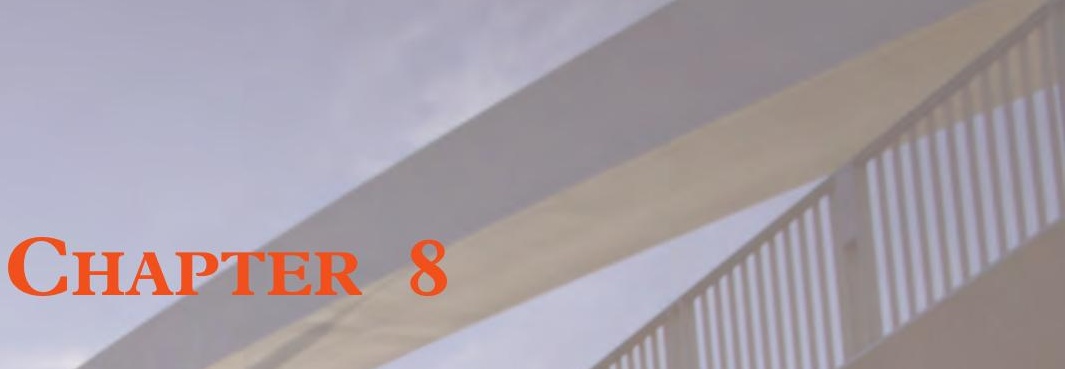

Dietary protein digestion and absorption are impaired during acute post-exercise recovery in healthy young men

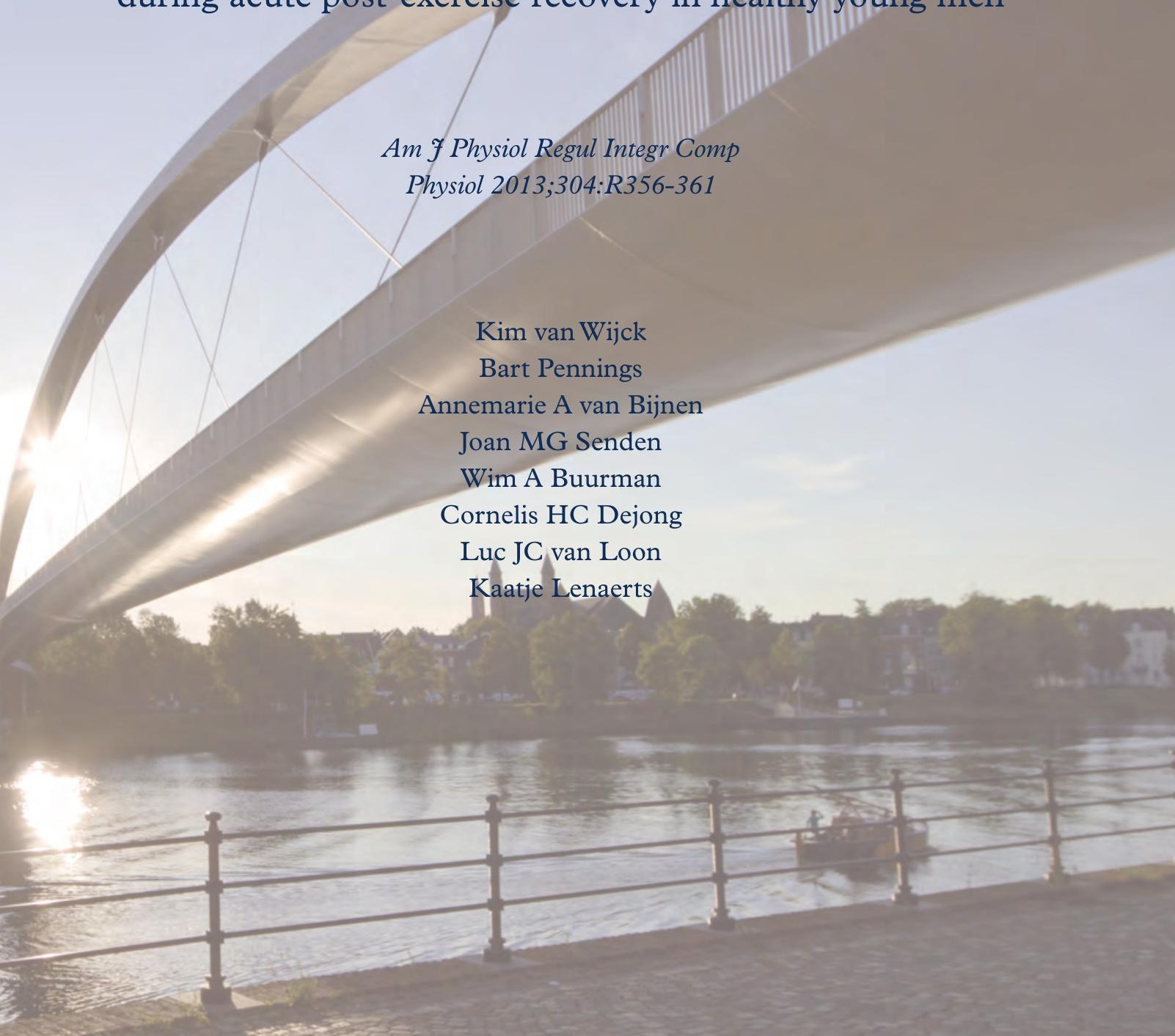




\begin{abstract}
Previously, we demonstrated that exercise can cause small intestinal injury, leading to loss of gut barrier function. The functional consequences of such exercise-induced intestinal injury on subsequent food digestion and absorption are unclear. The present study determined the impact of resistance-type exercise on small intestinal integrity and in vivo dietary protein digestion and absorption kinetics.
\end{abstract}

Twenty four young males ingested $20 \mathrm{~g}$ specifically produced intrinsically L- $\left[1-{ }^{13} \mathrm{C}\right]$ phenylalanine-labeled protein at rest or after performing a single bout of resistance-type exercise. Continuous intravenous infusions with $\mathrm{L}$ - $\left[\right.$ ring- ${ }^{2} \mathrm{H}_{5}$ ] phenylalanine were employed, and blood samples were collected regularly to assess in vivo protein digestion and absorption kinetics and to quantify plasma levels of intestinal fatty-acid binding protein (I-FABP) as a measure of small intestinal injury.

Plasma I-FABP levels were increased after exercise by $35 \%$, reaching peak values of $344 \pm 53$ $\mathrm{pg} / \mathrm{mL}$ compared to baseline $254 \pm 31 \mathrm{pg} / \mathrm{mL}(P<0.05)$. In resting conditions, I-FABP levels remained unchanged. Dietary protein digestion and absorption rates were reduced during postexercise recovery when compared with resting conditions $(P<0.001)$, with average peak exogenous phenylalanine appearance rates of $0.18 \pm 0.04$ vs. $0.23 \pm 0.03 \mathrm{mmol}$ phenylalanine.kg lean body $\mathrm{mass}^{-1} \cdot \mathrm{min}^{-1}$, respectively. Plasma I-FABP levels correlated with in vivo rates of dietary protein digestion and absorption $\left(\mathrm{R}_{\mathrm{S}}=-0.57, \mathrm{P}<0.01\right)$.

In conclusion, resistance-type exercise induces small intestinal injury in healthy, young men, causing impairments in dietary protein digestion and absorption kinetics during the acute postexercise recovery phase. To the best of our knowledge, this is first evidence that shows that exercise attenuates dietary protein digestion and absorption kinetics during acute post-exercise recovery. 


\section{INTRODUCTION}

The gut is challenged with two major functions, digestion and absorption of nutrients and the maintenance of an intact barrier against potentially harmful luminal compounds. Exercise is known to cause gastrointestinal (GI) injury and gut barrier dysfunction, reflected by increased small intestinal permeability $(20,23,24)$, bacterial translocation and inflammation after exercise $(6,15)$. We demonstrated that one hour of exercise induced small intestinal injury, leading to gut barrier dysfunction in healthy young athletes (30). These data gave rise to the intriguing question whether exercise-induced intestinal injury compromises the digestive and absorptive capacities of the GI tract.

Dietary protein ingestion following exercise has been reported to increase post-exercise muscle protein synthesis rates, thereby promoting early recovery after strenuous exercise (1). Impairments in dietary protein digestion and absorption may reduce the appearance rate of dietary protein-derived amino acids in the circulation (12), thereby lowering the postprandial availability of these amino acids for muscle damage repair and reconditioning. As muscle protein synthesis is maximally stimulated during acute post-exercise recovery, a rapid supply of exogenous protein is required to allow muscle protein synthesis rates to increase $(1,16,18,19)$. The latter is important to athletes as well as patients with chronic diseases to allow proper adaptation to each successive exercise bout within exercise intervention programs designed to improve performance and health. Impairments in food digestion and nutrient absorption during and immediately after exercise may jeopardize the adaptive response to the combined effect of exercise and proper nutrition $(1,14,16)$.

We hypothesized that exercise-induced intestinal injury impairs dietary protein digestion and absorption kinetics during acute post-exercise recovery. The present study was designed to determine the impact of a single bout of resistance type exercise on intestinal damage and on the subsequent digestive and absorptive function of the GI tract. Small intestinal injury and dietary protein digestion and absorption kinetics were assessed following ingestion of a single meal-like bolus of dairy protein at rest and after a single bout of resistance-type exercise in 24 healthy young males. By combining the use of specifically produced intrinsically $\mathrm{L}-\left[1-{ }^{13} \mathrm{C}\right]$ phenylalanine labeled dairy protein with continuous intravenous $\mathrm{L}-\left[\mathrm{ring}_{-}{ }^{2} \mathrm{H}_{5}\right]$ phenylalanine infusion we assessed in vivo dietary protein digestion and absorption kinetics $(26,29)$. This study shows novel evidence that resistance-type exercise induces small intestinal damage and impairs in vivo dietary protein digestion and absorption during acute post-exercise recovery in young, healthy men. 


\section{METHODS AND MATERIALS}

\section{Ethical approval}

The current study was approved by the medical ethical committee of Maastricht University Medical Centre+, and conducted in accordance with the Declaration of Helsinki (revised version, October 2008, Seoul). The study is part of a trial registered at clinicaltrials.gov as NCT00557388.

\section{Participant characteristics}

Twenty-four young, healthy male volunteers ( $21 \pm 1$ years of age) were included in the current study. The volunteers had no abdominal complaints during daily activities, had not taken any medication for at least one month prior to participation, had no history of GI disease, and had had no abdominal surgery. All participants of the current study were recreationally active people, reporting to spend 5-10 hours a week on physical exercise. Participants were randomly assigned to the rest or exercise experiment. Participant characteristics, presented in Table 1, did not differ between groups. All participants were informed about the nature and risks of the experiments before written consent was obtained.

Table 1: Baseline characteristics of the healthy male participants.

\begin{tabular}{lccc} 
& Rest & Exercise & $p$ value \\
\hline Group size (no. of participants) & 12 & 12 & 0.86 \\
Age (years) & $21 \pm 1$ & $21 \pm 1$ & 0.85 \\
Weight (kg) & $76.2 \pm 3.6$ & $77.0 \pm 2.1$ & 0.61 \\
Body Mass Index $\left(\mathrm{kg} / \mathrm{m}^{2}\right)$ & $23.4 \pm 1.0$ & $22.8 \pm 0.5$ & 0.56 \\
Body fat percentage $(\%)$ & $15.2 \pm 0.9$ & $15.9 \pm 0.9$ & 0.95 \\
Fat free mass (kg) & $64.5 \pm 2.8$ & $64.7 \pm 1.6$ & 0.55 \\
Leg volume (L) & $8.7 \pm 0.5$ & $9.1 \pm 0.3$ & 0.71 \\
Plasma glucose (mmol/L) & $5.1 \pm 0.1$ & $5.2 \pm 0.1$ & 0.60 \\
Plasma insulin (mU/L) & $10.4 \pm 0.7$ & $9.7 \pm 1.0$ & 0.21 \\
Blood HbA $(\%)$ & $5.4 \pm 0.1$ & $5.3 \pm 0.1$ & 0.58 \\
HOMA-IR & $2.4 \pm 0.2$ & $2.2 \pm 0.2$ & 0.56 \\
1 RM leg press (kg) & $254 \pm 17$ & $243 \pm 8$ & 0.96 \\
RM leg extension (kg) & $122 \pm 6$ & $121 \pm 5$ & \\
\hline
\end{tabular}

Data are presented as mean \pm SEM. $\mathrm{HbA}_{1 \mathrm{C}}$, glycated hemoglobin; HOMA-IR, homeostasis model of insulin resistance; $1 \mathrm{RM}$, one-repitition maximum leg strength.

\section{Pre-test arrangements}

Prior to the experiments, body composition analysis was performed using a dual energy X-ray absorptiometry scan (DXA ${ }^{\mathrm{TM}}$, Hologic Inc., Bedford, MA), and leg volume was determined as described previously (31). Electrocardiography was performed at rest and during exercise to exclude any heart failure in the selected volunteers. In addition, the subject's maximal strength 
(one repetition maximum, $1 \mathrm{RM}$ ) was estimated after a session of multiple repetitions of leg press and leg extension exercises (21). Subsequently, during a second session, the subject's true 1RM was determined by setting the load to $90-95 \%$ of the estimated $1 \mathrm{RM}$, and increased after each successful lift. An interval of 7 days or more was scheduled between the screening sessions and the experimental day.

Participants maintained normal activities of daily living, but refrained from strenuous physical activity for 3 days prior to the experiments. The evening before the experimental day, participants received a standardized meal $(33 \pm 2 \mathrm{~kJ} / \mathrm{kg}$ body weight, providing $55 \%$ of energy as carbohydrate, $15 \%$ as protein and $30 \%$ as fat) at $~ 8: 00$ P.M. after which they remained fasted.

\section{Study design and sampling}

In the morning, a catheter was placed in the participant's forearm vein for stable-isotope infusion. A second catheter was inserted in a heated dorsal hand vein of the contralateral arm and placed in hot box heated to $60^{\circ} \mathrm{C}$ for arterialized blood sampling. Blood was collected before ( $\left.-30 \mathrm{~min}\right)$, immediately post-exercise/rest ( $0 \mathrm{~min}$ ), and 15, 30, 45, 60, 75, 90, 105 and 120 minutes postexercise/rest to enable thorough analysis of enterocyte integrity and protein digestion and absorption especially in the immediate post-exercise period. Blood was sampled in EDTA tubes, centrifuged at $1000 \mathrm{~g}$ for $5 \mathrm{~min}$ at $4^{\circ} \mathrm{C}$, and stored until analysis at $-80^{\circ} \mathrm{C}$. After baseline sampling ( $\mathrm{t}=-120 \mathrm{~min}$ ), a single intravenous (IV) dose of the tracer, L-[ring- ${ }^{2} \mathrm{H}_{5}$ ] phenylalanine $(2 \mu \mathrm{mol} / \mathrm{kg}$ ) was infused to prime the plasma phenylalanine pool. Subsequently, the continuous tracer infusion was started at a rate of $0.044 \pm 0.001 \mu \mathrm{mol} \cdot \mathrm{kg}^{-1} \cdot \mathrm{min}^{-1}$, after which the participants rested in upright position for 90 minutes. Subjects then either performed a 30-minute standardized exercise protocol or remained seated for another 30 minutes of rest according to random assignment. The exercise protocol comprised a 5-minute self-paced cycling bout at $1.4 \pm 0.1 \mathrm{~W} / \mathrm{kg}$ body weight at a pace of 70-90 rotations per minute, combined with 6 sets of 10 repetitions on a horizontal leg press machine (Technogym BV, Rotterdam, the Netherlands) and 6 sets of 10 repetitions on a leg extension machine (Technogym BV). Each first two sets were performed at $40 \%$ of the subject's $1 \mathrm{RM}$. Sets 3-4 and 5-6 were performed at $55 \%$ and $75 \%$ of $1 \mathrm{RM}$, respectively, with a 2 -minute rest interval between sets.

After baseline sampling $(\mathrm{t}=0 \mathrm{~min})$, subjects received a single $250-\mathrm{mL}$ bolus flavored drink containing $20 \mathrm{~g}$ intrinsically L- $\left[1-{ }^{13} \mathrm{C}\right]$ phenylalanine-labeled casein protein. The latter was obtained by infusing a Holstein cow with large amounts of $\mathrm{L}-\left[1-{ }^{13} \mathrm{C}\right]$ phenylalanine and collecting the milk produced by this cow (29). The L- $\left[1-{ }^{13} \mathrm{C}\right]$ phenylalanine enrichment in the purified casein fraction of the cow milk averaged 29.2 moles percent excess. The drink met all specifications for safe human consumption. 


\section{Assessment of small intestinal injury}

To determine whether resistance-type exercise leads to small intestinal injury, concentrations of human Intestinal Fatty Acid Binding Protein (I-FABP) were analyzed by an enzyme linkedimmunosorbent assay (ELISA) in plasma samples collected before and after exercise. No samples were obtained during the short exercise bout. In short, 96-well plates (Greiner Microlon F, Greiner Bio-One GmbH, Frickenhausen, Germany) were coated overnight with anti-I-FABP immunoglobulin $\mathrm{G}(\mathrm{IgG})$ in a $2.5 \mu \mathrm{g} / \mathrm{ml}$ concentration in phosphate-buffered saline (PBS) at $4^{\circ} \mathrm{C}$.

Free sites were blocked with $1 \%$ bovine serum albumin (BSA) in PBS for one hour at room temperature. Subsequently, plasma samples and human recombinant I-FABP, used to produce standard calibration curves, were allowed to incubate for 1 hour at room temperature, after which $0.5 \mu \mathrm{g} / \mathrm{ml}$ of biotinylated anti-I-FABP IgG in $0.1 \%$ BSA-0.05\% Tween 20-PBS was added to the plates and left to incubate for 1 hour at room temperature. Horseradish peroxidase-streptavidin conjugate (Zymed Laboraties Inc., San Francisco, CA) in 0.1\% BSA-PBS and 3,3,5,5tetramethylbenzidine (Kirkegaard \& Perry Laboratories, Gaithersburg, MD) in substrate buffer were used to induce a color reaction, which was stopped after approximately 35 minutes by adding $\mathrm{H}_{2} \mathrm{SO}_{4}$. Color intensity was measured using an ELISA reader set at $450 \mathrm{~nm}$. The detection window was 12.5 to $800 \mathrm{pg} / \mathrm{mL}$.

\section{Assessment of dietary protein-derived phenylalanine uptake}

Plasma amino acid concentrations were determined by HPLC after deproteinization. The latter was done on ice with $10 \mathrm{mg}$ of dry 5-sulfosalicylic acid, which was mixed with $100 \mu \mathrm{L}$ of plasma and centrifuged, after which clear supernatant was collected. HPLC was used to determine plasma amino acid concentrations after precolumn derivatization with $o$-phtaldialdehyde (28). Plasma phenylalanine enrichment was measured using its $t$-butyldimethylsilyl derivative, and its ${ }^{13} \mathrm{C}$ and ${ }^{2} \mathrm{H}$ enrichments were determined by electron ionization gas chromatography in combination with mass spectrometry (GC-MS; 6890 GC/5973N MSD; Agilent, Little Falls, DE). Molecular masses 336, 337 and 341 were assessed for unlabeled phenylalanine, $1-{ }^{13} \mathrm{C}$ phenylalanine, and ring- ${ }^{2} \mathrm{H}_{5}$ phenylalanine, respectively (32). Standard regression curves were used in all isotopic enrichment analyses. Enrichments were calculated according to Biolo et al (2) to correct for the presence of the ${ }^{13} \mathrm{C}$ and ${ }^{2} \mathrm{H}_{5}$ isotopes. 


\section{Calculations}

The total and exogenous (exo) rate of phenylalanine appearance $\left(R_{a}\right)$ and plasma availability of phenylalanine ( Phe $_{\text {plasma }}$ ) were determined by modified Steele equations $(5,8)$. These parameters were calculated as:

$$
\begin{aligned}
& \text { Total } \mathrm{R}_{\mathrm{a}}=\frac{F-p \mathrm{~V} \cdot C(t) \cdot \mathrm{d} E_{i v} / \mathrm{d} t}{E_{i v}(\mathrm{t})} \\
& \text { Exo } \mathrm{R}_{\mathrm{a}}=\frac{\text { Total } \mathrm{R}_{\mathrm{a}} \cdot E_{p o}(t)+p \mathrm{~V} \cdot \mathrm{d} E_{i v} / \mathrm{d} t}{E_{p r o t}} \\
& \text { Phe }_{\text {plasma }}=\frac{\mathrm{AUC}_{\text {Exo } R a} \cdot 100}{\text { Phe }_{\text {prot }}}
\end{aligned}
$$

Where $F$ is the intravenous (iv) tracer infusion rate, $p \mathrm{~V}$ is the distribution volume for phenylalanine ( $p \mathrm{~V}=0.125), C(\mathrm{t})$ is the mean plasma phenylalanine concentration between two consecutive time points, $\mathrm{d} E_{i v} / \mathrm{d} t$ is the time-dependent variation of plasma phenylalanine enrichment derived from the iv tracer, and $E_{i v}(t)$ is the mean plasma phenylalanine enrichment from the iv tracer between two consecutive time points. $E_{p o}$ is the mean plasma phenylalanine enrichment form the oral tracer, $\mathrm{d} E_{p o} / \mathrm{d} t$ represents the time-dependent variations in plasma enrichment from the oral tracer, $E_{\text {prot }}$ is the L- $\left[1-{ }^{13} \mathrm{C}\right]$ phenylalanine enrichment in the dietary protein, $\mathrm{Phe}_{\text {prot }}$ is the amount of dietary phenylalanine ingested. $\mathrm{AUC}_{\mathrm{Exo} R a}$ represents the area under the curve (AUC) of Exo $\mathrm{R}_{a}$, corresponding to the amount of dietary phenylalanine appearing in the circulation over the $6 \mathrm{~h}$ period after intake of the drink.

\section{Statistical analysis}

Statistical analysis was performed using GraphPad Prism (Version 5.00, GraphPad Software for Windows, San Diego California, USA). All data are presented as mean \pm standard error of the mean $( \pm$ SEM). Normal distribution was tested using the D'Agostino and Pearson omnibus test. Paired data were analyzed by Friedman test/Wilcoxon signed rank test or repeated-measures analysis of variance/Student's paired t-test. Unpaired variables were assessed by Mann-Whitney U test or Student's unpaired t-test. Correlation analysis was performed and expressed using Spearman correlation coefficient $\left(\mathrm{R}_{\mathrm{S}}\right)$. Linear regression was used to visualize the correlation. Correlations between plasma I-FABP levels and protein digestion and absorption rates were determined in samples collected immediately after rest/exercise and 45 minutes after rest/exercise, respectively, since we expected this to be the time interval in which the largest differences could be observed. A $P$-value $<0.05$ was considered statistically significant. 


\section{RESULTS}

\section{Small intestinal injury following resistance-type exercise}

The 12 participants assigned to the exercise experiment all completed the 30-min standardized exercise protocol, consisting of 5-min cycling and resistance-type leg exercises. Intensity of cycling and leg exercises were based on the individual's body weight and maximal strength, respectively. Plasma levels of I-FABP increased from $254 \pm 31 \mathrm{pg} / \mathrm{ml}$ at baseline to $344 \pm 53 \mathrm{pg} / \mathrm{ml}$ measured directly after the last leg exercise, i.e. an increase of $35.4 \%$ from baseline $(P<0.05)$, while I-FABP levels did not change in resting conditions (Figure 1). Plasma I-FABP levels after exercise, depicted as a percentage from baseline, were increased compared to levels observed in the resting control treatment $(P<0.05$; Figure 1).

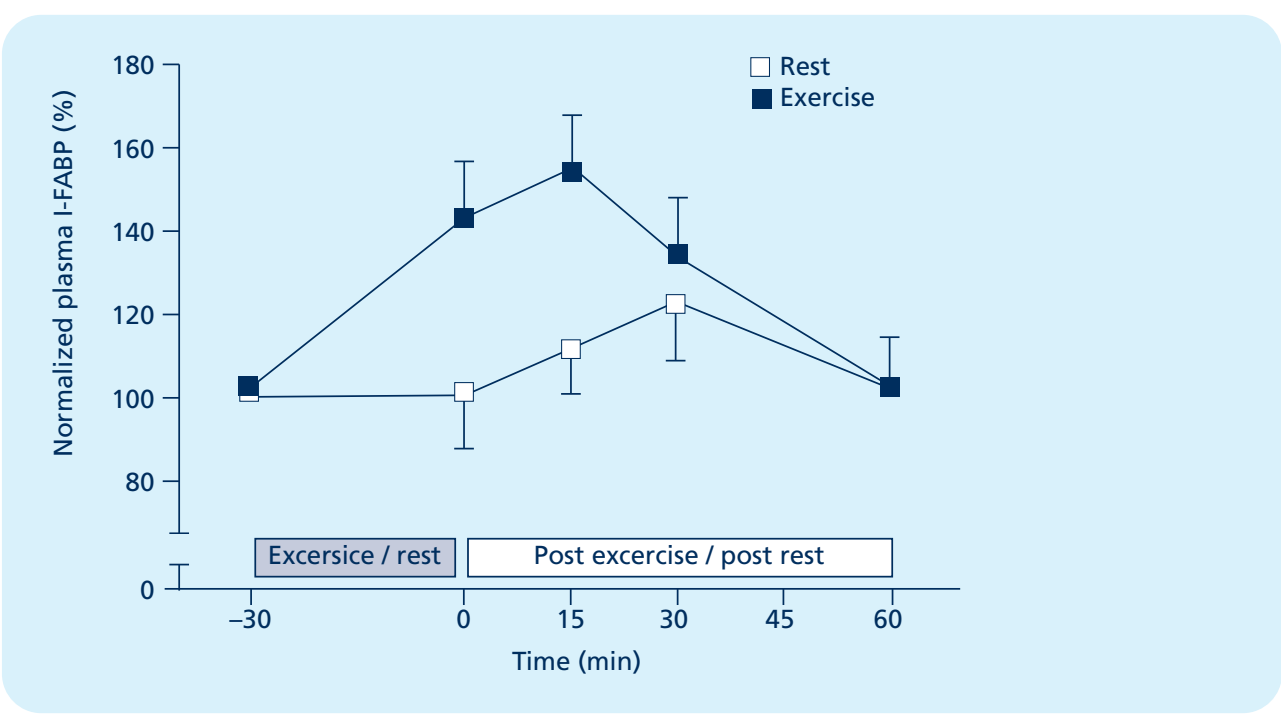

Figure 1: Resistance-type exercise increases plasma I-FABP levels

Thirty minutes of resistance-type exercise increased plasma I-FABP levels when compared with rest $(P<0.01)$. Solid squares represent post-exercise data and open squares represent data obtained from athletes at rest. Squares represent mean data, with the SEM as black lines.

\section{Uptake of amino acids from ingested dietary protein}

Digestion and absorption of dietary L- $\left[1-{ }^{13} \mathrm{C}\right]$ phenylalanine-labeled protein was studied in 12 athletes after 30 minutes at rest and in 12 athletes after a single bout of exercise. Both in resting and in post-exercise conditions, a trend towards increased plasma I-FABP levels was associated with protein intake (total increase of ca. $17 \%$ compared with pre-intake levels; $n=24, P=0.06$; Figure 1). Oral intake of the labeled dietary protein increased plasma phenylalanine levels both in both the resting and post-exercise condition, reaching peak levels $30 \mathrm{~min}$ after protein intake 


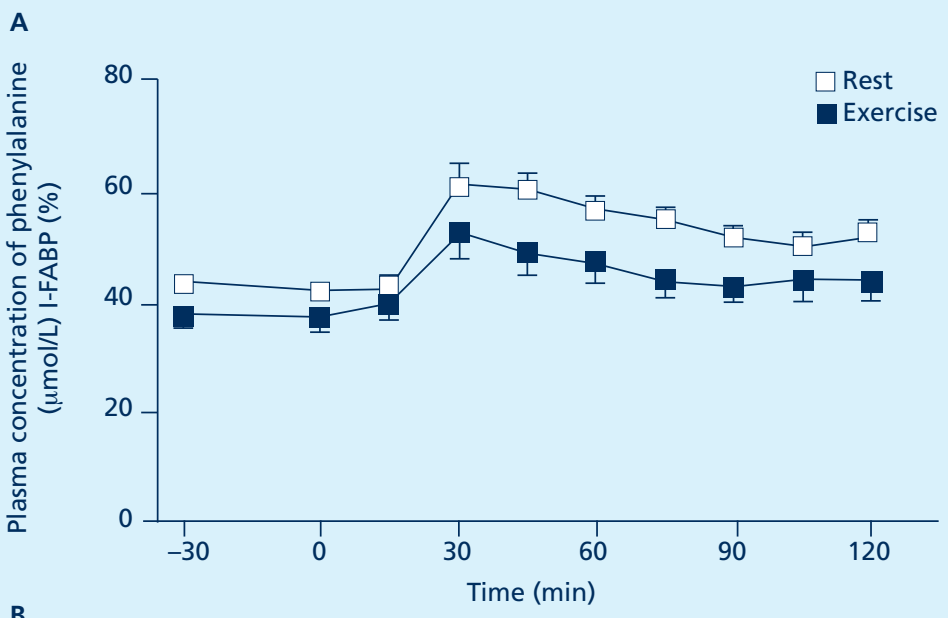

B

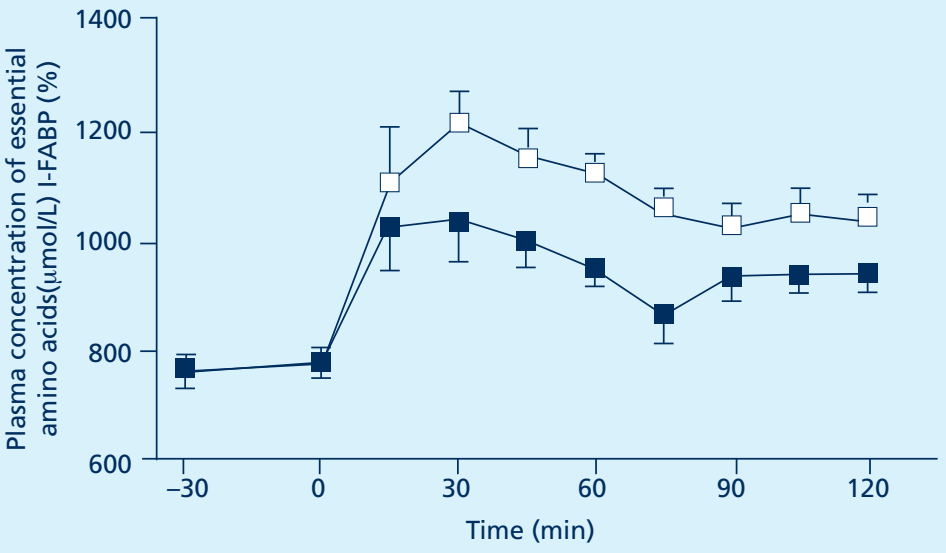

Figure 2: Plasma phenylalanine (A) and total essential amino acid (B) concentrations

A) Postprandial increases in plasma phenylalanine levels were more pronounced at rest when compared with postexercise conditions $(P<0.0001)$. B) Plasma essential amino acid concentrations increased after protein ingestion at rest and after exercise, but post-exercise concentrations were lower when compared with values observed during resting conditions $(P<0.0001)$. Solid squares represent post-exercise data and open squares represent data obtained from athletes at rest. Data represent mean \pm SEM.

(Figure $2 A$ ). Plasma phenylalanine concentrations were higher in resting compared with postexercise conditions $(P<0.0001$; Figure $2 \mathrm{~A})$.

Ingestion of the labeled dietary protein did not only elevate the circulating levels of phenylalanine, but also increased plasma concentrations of other essential amino acids both in resting and post-exercise conditions (Figure $2 \mathrm{~B}$ ). In line with the phenylalanine data, the postprandial rise in plasma essential amino acid levels was attenuated in the post-exercise situation when compared to the resting condition $(P<0.0001$; Figure $2 B)$. Post-prandial plasma non-essential amino acid concentrations were not significantly different between resting and post-exercise 
A

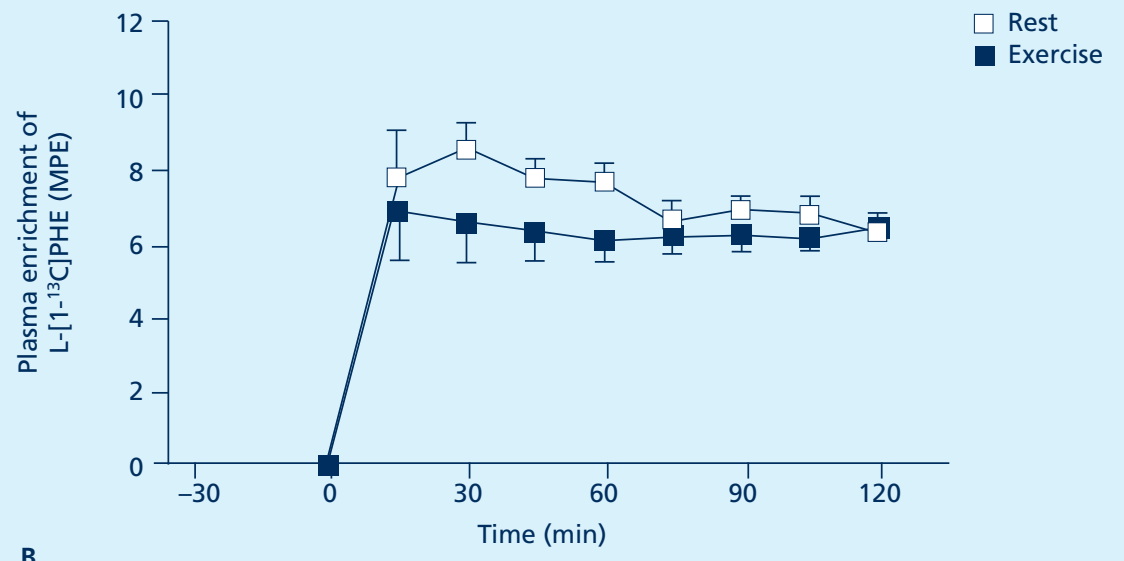

B

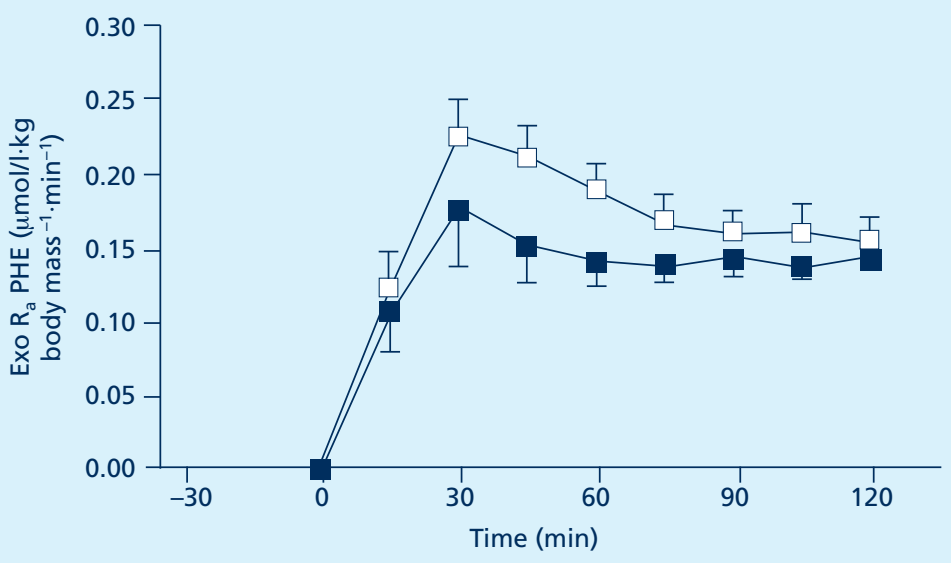

Figure 3: Plasma $L-\left[1{ }^{13} C\right]$ phenylalanine enrichment $(A)$ and exogenous phenylalanine rate of appearance (B)

A) Plasma L- $\left[1-{ }^{13} \mathrm{C}\right]$ phenylalanine enrichments were more pronounced in resting conditions compared to postexercise $(P<0.05)$ B) The dietary phenylalanine appearance rate was decreased in the $0-120$ min post-exercise recovery period when compared with resting conditions $(P<0.001)$. Solid squares represent post-exercise data and open squares represent data obtained from athletes at rest. Data represent mean \pm SEM. MPE, moles percent excess.

conditions (data not shown). In the post-exercise situation, plasma L- $\left[1-{ }^{13} \mathrm{C}\right]$ phenylalanine enrichments were lower when compared to resting conditions $(P<0.05$; Figure $3 A)$. In line, a trend towards a lower $\mathrm{AUC}_{0-120 \mathrm{~min}}$ of Exo $\mathrm{R}_{a}$ was observed after exercise when compared with resting conditions $\left(16 \pm 2.0\right.$ vs $20 \pm 1.5 \mathrm{mmol}$ phenylalanine. $\mathrm{kg}$ body mass ${ }^{-1} \cdot \mathrm{min}^{-1}$, respectively; $P=0.09$ ). 


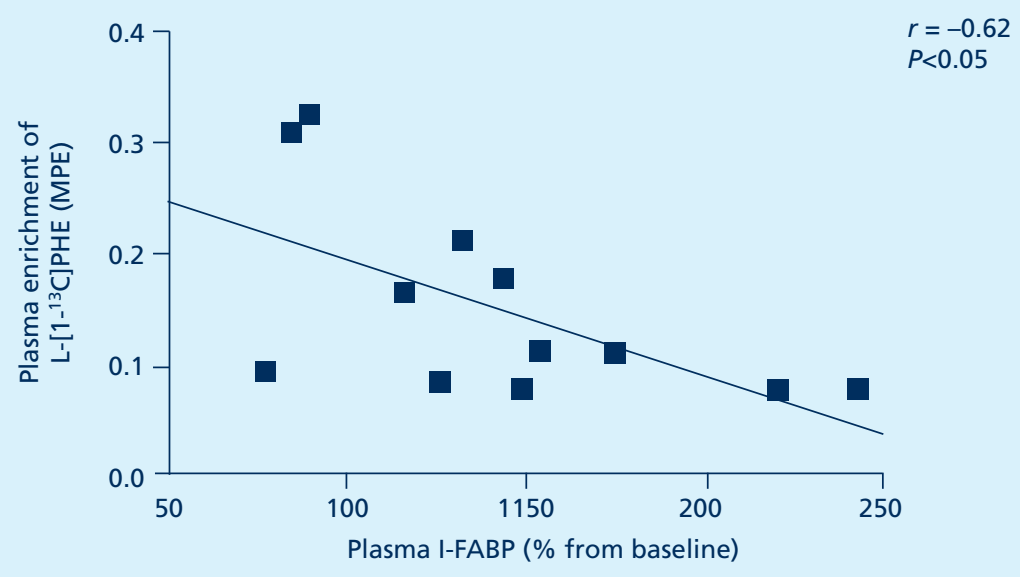

Figure 4: Correlation of plasma I-FABP levels with exogenous Phe rates of appearance

Exercise-induced small intestinal injury, reflected by plasma I-FABP levels, correlated negatively with in vivo dietary protein digestion and absorption rates $\left(\mathrm{R}_{\mathrm{S}}=-0.62, n=12, p<0.05\right.$ at 45 minutes $)$.

Following protein consumption, plasma exogenous phenylalanine appearance rates increased both in resting and post-exercise conditions, reaching peak levels 30-45 minutes after protein ingestion (Figure 3B). Interestingly, plasma phenylalanine appearance rates derived from the ingested dietary protein were lower during post-exercise recovery when compared with resting conditions. Exogenous phenylalanine plasma appearance rates peaked at $0.18 \pm 0.04 \mathrm{mmol}$ phenylalanine.kg lean body $\mathrm{mass}^{-1} \cdot \mathrm{min}^{-1}$ after exercise compared with $0.23 \pm 0.03 \mathrm{mmol}$ phenylalanine $\cdot \mathrm{kg}$ lean body $\mathrm{mass}^{-1} \cdot \mathrm{min}^{-1}$ at rest (Figure $3 B$ ).

\section{Exercise-induced intestinal injury attenuates post-exercise dietary protein digestion and absorption kinetics}

Changes in plasma I-FABP levels at rest or immediately after exercise correlated with in vivo dietary protein digestion and absorption rates $\left(\mathrm{R}_{\mathrm{S}}=-0.57, n=24, p<0.01\right.$ at 45 minutes).

Specific analysis of the subset of exercising participants revealed a correlation between changes in plasma I-FABP levels with in vivo dietary protein digestion and absorption rates $\left(\mathrm{R}_{\mathrm{S}}=-0.62\right.$, $n=12, p<0.05$ at 45 minutes; Figure 4). A correlation was observed between the total peak area of plasma I-FABP levels and total exogenous phenylalanine uptake $\left(\mathrm{R}_{\mathrm{S}}=-0.41, n=24, P<0.05\right)$. The latter correlation was also found if only the subset of exercising participants was analyzed $\left(\mathrm{R}_{\mathrm{S}}=-0.62, n=12, p<0.05\right.$ at 45 minutes). 


\section{DISCUSSION}

The current study shows that resistance-type exercise increases plasma I-FABP levels, reflecting small intestinal cellular injury. In line, postprandial dietary protein digestion and absorption kinetics are shown to be attenuated during acute post-exercise recovery. Plasma levels of I-FABP correlated with the appearance of the dietary protein-derived amino acids in the circulation, indicating that exercise-induced small intestinal injury impairs dietary protein digestion and absorption kinetics during acute post-exercise recovery.

Previously, we demonstrated that exercise-induced small intestinal injury is caused by splanchnic hypoperfusion (30). Splanchnic hypoperfusion during exercise is a result of rapid redistribution of blood from the abdominal region towards the active muscle groups (7), and is known to predominantly affect the mature enterocytes at the upper half of the intestinal villi. The reason for the susceptibility of these cells to hypoperfusion is the countercurrent exchange mechanism in the villi resulting in a low-flow and low-oxygen environment at the top of the villi $(3,4)$. The reduction of the splanchnic blood flow during exercise contributes to the physiological low-flow state of these enterocytes, further depriving the cells from nutrients and oxygen. I-FABP is a small protein present in the susceptible mature enterocytes, and is rapidly released upon cellular injury due to hypoperfusion and ischemia $(10,13)$. The noted rise in I-FABP during exercise found in this study suggests the occurrence of ischemia-induced injury. These observations are in line with previous data showing a twofold increase in baseline I-FABP levels after a single 60minute session of endurance-type exercise (30). Similar plasma I-FABP levels have been reported in patients with splanchnic hypoperfusion during non-abdominal surgery (increase of $\sim 60 \%$ from pre-surgical levels) (11) and in trauma patients brought into the emergency room (9). These data imply that the observations in the present study are not only relevant for athletes but may also be of important clinical relevance for more compromised patient groups.

Interestingly, a trend towards increasing plasma I-FABP levels was observed during the first 15 minutes after consuming a single 20 -g bolus of dietary protein, both in rest and after exercise, suggesting that protein intake slightly disturbs enterocyte integrity. This observation might be explained by the fact that the presence of food in the GI tract rapidly increases the need for splanchnic blood flow (27). We speculate that in the short time period directly after food intake, splanchnic blood flow may not be adequate, leading to minor GI compromise. Nonetheless, this rapid post-prandial increase in plasma I-FABP levels is relatively small when compared with the substantial 40-100\% increase in I-FABP levels observed after resistance-type or endurance-type exercise.

The production and use of intrinsically L- $\left[1-{ }^{13} \mathrm{C}\right]$ phenylalanine labeled dairy protein in combination with continuous intravenous infusion of the tracer $\mathrm{L}-\left[\mathrm{ring}-{ }^{2} \mathrm{H}_{5}\right]$ phenylalanine in this study provides a unique opportunity to assess dietary protein digestion and absorption in an in vivo human model $(26,29)$. Immediately after protein ingestion, protein-derived phenylalanine 
appeared rapidly in the circulation both at rest and after exercise. The post-prandial rise in plasma $1-{ }^{13} \mathrm{C}$ Phenylalanine appearance rate was attenuated in the acute post-exercise recovery period when compared with resting conditions. Uptake rates were lower after exercise than at rest, leading to reduced protein uptake during acute post-exercise recovery. In line, lower postprandial increases in plasma essential amino acid concentrations were observed after resistance-type exercise when compared with resting conditions. Altogether, these data show that dietary protein digestion and absorption are compromised during acute post-exercise recovery. The latter is of considerable interest as dietary protein digestion and absorption kinetics are at least partly responsible for the post-prandial muscle protein synthetic response to food intake $(17,25)$. However, despite diminished dietary protein digestion and absorption, postprandial muscle protein fractional synthesis rates were found to increase after exercise compared to rest in this population (25). In addition, studies showed that especially in the immediate postexercise period, protein intake stimulates muscle protein synthesis compared with late protein intake (19). Hence, we do not believe that protein intake should be delayed based on the current findings; but, since even small changes can make the difference between winning and losing in athletic competition, it may be useful to determine whether such a difference in absorption found in this study has a meaningful effect on postexercise net muscle protein balance. Because a more rapid supply of amino acids following exercise has previously been shown to maximize postexercise muscle protein synthesis rates and improve the skeletal muscle adaptive response to more prolonged exercise training, new insights on gastrointestinal functioning may be used for fine-tuning the nutritional strategies to meet both gastrointestinal and muscle capacity. Our present findings indicate that dietary protein digestion and absorption are compromised in the post-exercise recovery phase. In agreement, we previously demonstrated that exercise especially affects the proximal part of the GI tract (30), which is the primary location of dietary protein digestion and absorption (22). To further clarify the effects of exercise on post-exercise dietary protein uptake, the relation between exercise-induced intestinal injury and plasma appearance of the protein-derived amino acids was studied. Data revealed a strong, negative relation between plasma I-FABP levels and plasma appearance rates of exogenous phenylalanine. The presence of such a significant correlation between plasma I-FABP levels and in vivo dietary protein digestion and absorption kinetics implies that the extent of small intestinal injury measured by I-FABP is a good reflection of the impact of exercise on intestinal function with respect to dietary protein digestion and absorption. Determination of plasma I-FABP levels may be used to obtain valuable information on gut integrity and gut function of athletes and patients during training sessions and during exercise intervention programs, respectively. This may be of particular interest in more compromised patient populations that are subjected to a lifestyle intervention or rehabilitation program. As gut function is generally compromised in the elderly population it may be of considerable interest to also assess the impact of exercise on gut function and the subsequent delivery of exogenous amino acids to allow post-exercise muscle protein synthesis in an elderly population. 
From this study we conclude that exercise-induced loss of enterocyte integrity attenuates dietary protein digestion and absorption, impairing dietary protein derived amino acid uptake during acute post-exercise recovery in healthy, young recreationally active individuals. These data implicate that work is needed to address the impact of exercise on GI integrity and GI function. Dietary strategies need to consider compromised gut function and food intake regimens should be modulated to allow proper nutrient delivery during exercise and subsequent acute post-exercise recovery. The latter could be of considerable interest to optimize post-exercise muscle reconditioning in both health and disease. 


\section{REFERENCES}

1. Beelen M, Burke LM, Gibala MJ, and van Loon LJ. Nutritional strategies to promote postexercise recovery. Int f Sport Nutr Exerc Metab 20: 515-532, 2010.

2. Biolo G, Tessari P, Inchiostro S, Bruttomesso D, Fongher C, Sabadin L, Fratton MG, Valerio A, and Tiengo A. Leucine and phenylalanine kinetics during mixed meal ingestion: a multiple tracer approach. Am f Physiol 262: E455-463, 1992.

3. Blikslager AT. Life in the gut without oxygen: adaptive mechanisms and inflammatory bowel disease. Gastroenterology 134: 346-348, 2008.

4. Blikslager AT, Moeser AJ, Gookin JL, Jones SL, and Odle J. Restoration of barrier function in injured intestinal mucosa. Physiological reviews 87: 545-564, 2007.

5. Boirie Y, Gachon P, Corny S, Fauquant J, Maubois JL, and Beaufrere B. Acute postprandial changes in leucine metabolism as assessed with an intrinsically labeled milk protein. Am f Physiol 271: E1083-1091, 1996.

6. Bosenberg AT, Brock-Utne JG, Gaffin SL, Wells MT, and Blake GT. Strenuous exercise causes systemic endotoxemia. F Appl Physiol 65: 106-108, 1988.

7. Bradley SE, Childs AW, Combes B, Cournand A, Wade OL, and Wheeler HO. The effect of exercise on the splanchnic blood flow and splanchnic blood volume in normal man. Clin Sci (Lond) 15: 457-463, 1956.

8. Dangin M, Guillet C, Garcia-Rodenas C, Gachon P, Bouteloup-Demange C, Reiffers-Magnani K, Fauquant J, Ballevre $\mathrm{O}$, and Beaufrere $\mathrm{B}$. The rate of protein digestion affects protein gain differently during aging in humans. The fournal of physiology 549: 635-644, 2003.

9. de Haan JJ, Lubbers T, Derikx JP, Relja B, Henrich D, Greve JW, Marzi I, and Buurman WA. Rapid development of intestinal cell damage following severe trauma: a prospective observational cohort study. Critical care 13: R86, 2009.

10. Derikx JP, Matthijsen RA, de Bruine AP, van Bijnen AA, Heineman E, van Dam RM, Dejong CH, and Buurman WA. Rapid reversal of human intestinal ischemia-reperfusion induced damage by shedding of injured enterocytes and reepithelialisation. PloS ONE 3: e3428, 2008.

11. Derikx JP, van Waardenburg DA, Thuijls G, Willigers HM, Koenraads M, van Bijnen AA, Heineman E, Poeze M, Ambergen T, van Ooij A, van Rhijn LW, and Buurman WA. New Insight in Loss of Gut Barrier during Major Non-Abdominal Surgery. PloS ONE 3: e3954, 2008.

12. Freeman HJ, Kim YS, and Sleisenger MH. Protein digestion and absorption in man. Normal mechanisms and protein-energy malnutrition. The American journal of medicine 67: 1030-1036, 1979.

13. Grootjans J, Lenaerts K, Derikx JP, Matthijsen RA, de Bruine AP, van Bijnen AA, van Dam RM, Dejong CH, and Buurman WA. Human intestinal ischemia-reperfusion-induced inflammation characterized: experiences from a new translational model. The American journal of pathology 176: 2283-2291, 2010.

14. Hawley JA, Tipton KD, and Millard-Stafford ML. Promoting training adaptations through nutritional interventions. F Sports Sci 24: 709-721, 2006

15. Jeukendrup AE, Vet-Joop K, Sturk A, Stegen JH, Senden J, Saris WH, and Wagenmakers AJ. Relationship between gastro-intestinal complaints and endotoxaemia, cytokine release and the acute-phase reaction during and after a long-distance triathlon in highly trained men. Clin Sci (Lond) 98: 47-55, 2000.

16. Koopman R, Saris WH, Wagenmakers AJ, and van Loon LJ. Nutritional interventions to promote post-exercise muscle protein synthesis. Sports medicine 37: 895-906, 2007.

17. Koopman R, Walrand S, Beelen M, Gijsen AP, Kies AK, Boirie Y, Saris WH, and van Loon LJ. Dietary protein digestion and absorption rates and the subsequent postprandial muscle protein synthetic response do not differ between young and elderly men. The fournal of nutrition 139: 1707-1713, 2009.

18. Levenhagen DK, Carr C, Carlson MG, Maron DJ, Borel MJ, and Flakoll PJ. Postexercise protein intake enhances whole-body and leg protein accretion in humans. Medicine and science in sports and exercise 34: 828$837,2002$.

19. Levenhagen DK, Gresham JD, Carlson MG, Maron DJ, Borel MJ, and Flakoll PJ. Postexercise nutrient intake timing in humans is critical to recovery of leg glucose and protein homeostasis. American journal of physiology Endocrinology and metabolism 280: E982-993, 2001.

20. Marchbank T, Davison G, Oakes JR, Ghatei MA, Patterson M, Moyer MP, and Playford RJ. The nutriceutical bovine colostrum truncates the increase in gut permeability caused by heavy exercise in athletes. A $m \mathcal{F}$ Physiol Gastrointest Liver Physiol 300: G477-484, 2011

21. Mayhew JL, Prinster JL, Ware JS, Zimmer DL, Arabas JR, and Bemben MG. Muscular endurance repetitions to predict bench press strength in men of different training levels. J Sports Med Phys Fitness 35: 108-113, 1995.

22. Nixon SE, and Mawer GE. The digestion and absorption of protein in man. 1. The site of absorption. Br F Nutr 24: 227-240, 1970. 
23. Oktedalen O, Lunde OC, Opstad PK, Aabakken L, and Kvernebo K. Changes in the gastrointestinal mucosa after long-distance running. Scand F Gastroenterol 27: 270-274, 1992.

24. Pals KL CR-T, Ryan AJ, Gisolfi CV. Effect of running intensity on intestinal permeability. F Appl Physiol 82: 571-576, 1997.

25. Pennings B, Koopman R, Beelen M, Senden JM, Saris WH, and van Loon LJ. Exercising before protein intake allows for greater use of dietary protein-derived amino acids for de novo muscle protein synthesis in both young and elderly men. Am f Clin Nutr 93: 322-331, 2011.

26. Pennings B, Pellikaan WF, Senden JM, van Vuuren AM, Sikkema J, and van Loon LJ. The production of intrinsically labeled milk and meat protein is feasible and provides functional tools for human nutrition research. Fournal of dairy science 94: 4366-4373, 2011.

27. Sieber C, Beglinger C, Jager K, and Stalder GA. Intestinal phase of superior mesenteric artery blood flow in man. Gut 33: 497-501, 1992.

28. van Eijk HM, Rooyakkers DR, and Deutz NE. Rapid routine determination of amino acids in plasma by highperformance liquid chromatography with a 2-3 microns Spherisorb ODS II column. F Chromatogr 620: 143$148,1993$.

29. van Loon LJ, Boirie Y, Gijsen AP, Fauquant J, de Roos AL, Kies AK, Lemosquet S, Saris WH, and Koopman $\mathrm{R}$. The production of intrinsically labeled milk protein provides a functional tool for human nutrition research. F Dairy Sci 92: 4812-4822, 2009.

30. van Wijck K, Lenaerts K, van Loon LJ, Peters WH, Buurman WA, and Dejong CH. Exercise-induced splanchnic hypoperfusion results in gut dysfunction in healthy men. PloS ONE 6: e22366, 2011.

31. Verdijk LB, Jonkers RA, Gleeson BG, Beelen M, Meijer K, Savelberg HH, Wodzig WK, Dendale P, and van Loon LJ. Protein supplementation before and after exercise does not further augment skeletal muscle hypertrophy after resistance training in elderly men. Am f Clin Nutr 89: 608-616, 2009.

32. Wolfe RR. Radioactive and stable isotope tracers in biomedicine: principles and practice of kinetic analysis. New York, NY: Wiley-Liss, 1992 


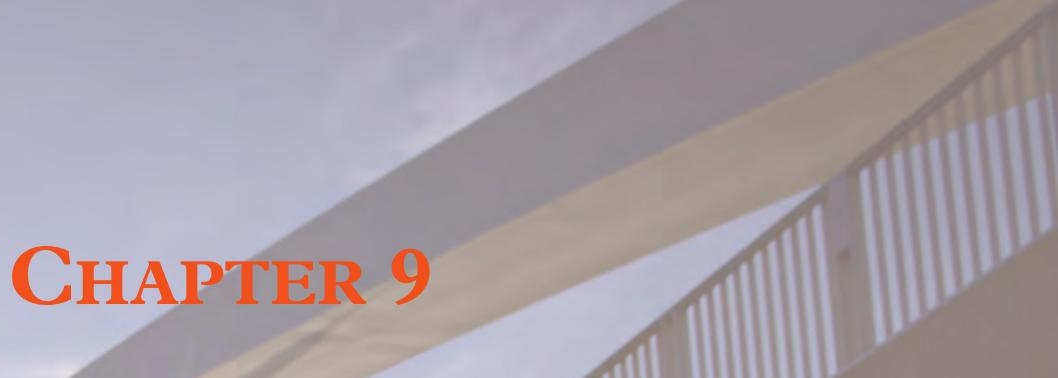

Aggravation of exercise-induced intestinal injury

by ibuprofen in athletes

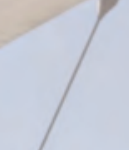

Med Sci Sports Exercise

Volume 44, Issue 12, 2257-2262, 2012

Kim van Wijck

Kaatje Lenaerts

Annemarie A van Bijnen

Bas Boonen

Luc JC van Loon

Cornelis HC Dejong

Wim A Buurman

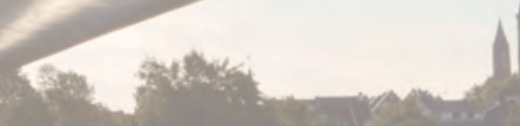

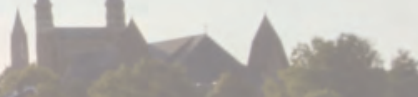




\section{ABSTRACT}

Non-steroidal anti-inflammatory drugs (NSAIDs) are commonly used by athletes to prevent anticipated exercise-induced pain, thereby putatively improving physical performance. However, these drugs may have potentially hazardous effects on the gastrointestinal (GI) mucosa during strenuous physical exercise. The aim of the current study was to determine the effect of oral ibuprofen administration prior to exercise on GI integrity and barrier function in healthy individuals.

Nine healthy, trained men were studied on four different occasions: 1) $400 \mathrm{mg}$ ibuprofen twice prior to cycling, 2) cycling without ibuprofen, 3) $400 \mathrm{mg}$ ibuprofen twice at rest, and 4) rest without ibuprofen intake. To assess small intestinal injury, plasma intestinal fatty acid binding protein (I-FABP) levels were determined, whereas urinary excretion of orally ingested multisugar test probes was measured using liquid chromatography and mass spectrometry to assess GI permeability.

Both ibuprofen consumption and cycling resulted in increased I-FABP levels, reflecting small intestinal injury. Levels were higher after cycling with ibuprofen than after cycling without ibuprofen, rest with ibuprofen, or rest without ibuprofen (peak I-FABP $875 \pm 137,474 \pm 74$, $507 \pm 103$, and $352 \pm 44 \mathrm{pg} / \mathrm{mL}$, respectively, $P<0.002)$. In line, small intestinal permeability increased, especially after cycling with ibuprofen $(0-2 \mathrm{~h}$ urinary lactulose/rhamnose $(\mathrm{L} / \mathrm{R})$ ratio 0.08(0.04-0.56) compared to 0.04(0.00-0.20), 0.05(0.01-0.07), and 0.01(0.01-0.03), respectively), reflecting loss of gut barrier integrity. Interestingly, the extent of intestinal injury and barrier dysfunction correlated significantly $\left(\mathrm{R}_{\mathrm{S}}=0.56, P<0.001\right)$.

In conclusion, this is the first study to reveal that ibuprofen aggravates exercise-induced small intestinal injury and induces gut barrier dysfunction in healthy individuals. We conclude that NSAID consumption by athletes is not harmless and should be discouraged. 


\section{INTRODUCTION}

Non-steroidal anti-inflammatory drugs (NSAIDs) are commonly used by athletes to reduce existing or prevent anticipated musculoskeletal pain related to physical exercise, especially in the competitive season $(1,15)$. Athletes have reported the use of NSAIDs both during training and competition (16). It is generally assumed that NSAIDs improve athletic performance by enabling more frequent and more intensive training sessions, but no clear evidence exists. The reported prevalence of NSAID use among athletes differs widely between various sport types and ranges from $12 \%$ in cyclists to over $90 \%$ in professional soccer players $(16,29,32)$.

A major adverse effect of NSAIDs is their known tendency to cause gastrointestinal (GI) complications such as mucosal ulceration, bleeding, perforation, and the formation of diaphragmlike strictures $(2,35)$. Several mechanisms of action have been suggested to play a role in the development of these NSAID-induced complications: inhibition of cyclo-oxygenase (COX)-1 and reduction of local nitric oxide (NO) production via regulation of the NF-K-B pathway may impair perfusion of the upper GI tract (20), whereas COX-2 inhibition compromises immunomodulation, potentially resulting in the onset of an inflammatory response (4).

We previously demonstrated that one hour of exhaustive physical activity leads to small intestinal injury and short-term loss of gut barrier function in otherwise healthy individuals (33). In the light of such exercise-induced GI compromise, it seems that the hazardous effects of NSAIDs on the GI mucosa may put athletes at risk for more severe abdominal distress. We hypothesize that the combination of short-term NSAID consumption with the physiological exercise-induced splanchnic hypoperfusion may lead to aggravated intestinal injury in athletes. The current study shows that the use of NSAIDs intensifies exercise-induced small intestinal injury and loss of intestinal barrier integrity in healthy athletes.

\section{METHODS AND MATERIALS}

\section{Subjects' characteristics}

Power calculation performed prior to the start of the study revealed that a sample size of 9 subjects was required to achieve $80 \%$ statistical power and detect statistically significant differences in the current experimental setting. Therefore, nine healthy male cyclists or triathletes (age: $27 \pm 0.9$ years, body mass index: $20.6 \pm 0.6 \mathrm{~kg} / \mathrm{m}^{2}$ ) were selected to participate in the present study. Maximal workload capacity $\left(\mathrm{W}_{\max }\right)$ was $5.5 \pm 0.2 \mathrm{~W} / \mathrm{kg}$, with maximum heart rate at $192 \pm 2 \mathrm{bpm}$. All volunteers spent 3 to 10 hours per week performing endurance sports as part of their normal life style. The volunteers had no abdominal complaints during daily activities, had not taken any medication for at least one month prior to participation, had no history of GI disease, and had no history of abdominal surgery. Volunteers were informed about the nature and risks of the experiments. 
Written consent was obtained at least 5 days prior to the experiments. This study was approved by the medical ethics committee of Maastricht University Medical Centre+, and conducted in accordance with the Declaration of Helsinki (revised version, October 2008, Seoul).

\section{Pre-exercise arrangements}

$\mathrm{W}_{\max }$ was assessed prior to the experiments on a stationary cycle ergometer (Lode Excalibur, Groningen, The Netherlands) (18). Electrocardiographic monitoring (MAC 5500, GE Medical Systems, Freiburg, Germany) was performed to exclude cardiologic abnormalities such as exercise-induced cardiac ischemia or arrhythmia. An experienced cardiological resident interpreted the results and found no abnormalities. Participants maintained normal activities of daily living, but refrained from strenuous physical activity for the 2 days prior to the test days, and were not allowed to consume alcohol and artificial sweeteners the day prior to the experiments. Ibuprofen (400 mg, iso-butyl-propanoic-phenolic acid, GlaxoSmithKline, Brentford, Middlesex, United Kingdom) was ingested by the participants on the evening prior to the ibuprofen test days. An additional $400 \mathrm{mg}$ of ibuprofen was consumed on the morning of these test days, at 7:00 a.m., being 60 minutes before start of the experiments to mimic the normal use of ibuprofen by athletes.

\section{Study design and sampling}

All 9 subjects were tested consecutively in random order in 4 different situations: (1) during and after cycling after intake of ibuprofen, (2) during and after cycling without ibuprofen, (3) rest with prior intake of ibuprofen, (4) rest without prior ibuprofen intake. Time between test days was at least 7 days. All experiments were performed after an overnight fast. Upon arrival to the sports laboratory at 08:00 a.m., a catheter (20 Gauge, Braun, Melsungen, Germany) was placed in the participant's forearm vein to obtain blood samples, which were put into pre-chilled EDTA tubes (Vacucontainer, Becton Dickinson (BD), Helsingborg, Sweden) and kept on ice.

On test days that participants were assigned to cycling, participants started cycling at a workload of $150 \mathrm{~W}$ after collection of baseline plasma and urine samples. After 3 minutes, workload was increased to $70 \%$ of the individual's pre-assessed $\mathrm{W}_{\max }\left(70 \%\right.$ of $\mathrm{W}_{\max }$ was $268 \pm 9 \mathrm{~W}$, depicted as mean \pm SEM of the 9 participants). Subjects maintained pedal rates of at least $60 \mathrm{rpm}$, and workload was decreased by $25 \mathrm{~W}$ if participants were unable to maintain $60 \mathrm{rpm}$. Participants consumed tap water ad libitum, with a minimum of $50 \mathrm{~mL}$ and a maximum of $150 \mathrm{~mL}$ every 10 minutes. After 30 minutes of cycling, the test subjects ingested a $150-\mathrm{mL}$ multi-sugar drink to enable whole gut permeability analysis. A second urine sample was collected by the participants at 90 minutes after exercise. Resting test days were similar in setup, with the exception that the participants were tested during and after 1 hour of rest in supine position instead of during and after cycling. On all test days, blood and urine samples were centrifuged within 1 hour of collection at $4^{\circ} \mathrm{C}$ at $2300 \mathrm{x} g$ for 15 minutes, and stored at $-80^{\circ} \mathrm{C}$ until analysis. Analyses were performed when sample collection was complete for all participants, in order to perform 
analyses in consecutive runs. Any GI complains of the subjects during the test days were registered by the researcher, and subjects were contacted 2 days after the test day to assess the occurrence of GI complaints on the day following each test day.

\section{Assessment of small intestinal injury}

To evaluate the presence and the extent of small intestinal injury in the above-described situations, plasma concentrations of human Intestinal Fatty Acid Binding Protein (I-FABP) were determined by an analyst who was blinded for the specific test conditions. I-FABP is a $15-\mathrm{kD}$ cytosolic protein present especially in the mature enterocytes of the small intestine that rapidly diffuses through the interstitial space into the circulation upon enterocyte injury, making it an early and sensitive marker of small intestinal injury $(19,25)$. I-FABP was measured by an inhouse developed enzyme linked-immunosorbent assay (ELISA). In short, ELISA plates were coated with anti-I-FABP immunoglobulin G (IgG) overnight at $4^{\circ} \mathrm{C}$, free sites were blocked with $1 \%$ bovine serum albumin (BSA) in PBS. Plasma samples and human recombinant I-FABP for standard calibration curves were incubated at room temperature, after which biotinylated anti-I-FABP IgG was added. After washing, horseradish peroxidase-streptavidin conjugate (Zymed Laboraties Inc., San Francisco, CA) in 0.1\% BSA-PBS and 3,3,5,5-tetramethylbenzidine (Kirkegaard \& Perry Laboratories, Gaithersburg, MD) were added. The reaction was stopped and color intensity was measured with an ELISA reader at $450 \mathrm{~nm}$. The detection window of the I-FABP assay was 12.5 to $800 \mathrm{pg} / \mathrm{mL}$.

\section{Assessment of GI permeability}

GI permeability was determined as a measure of GI barrier integrity, using a multi-sugar test drink as described previously (34). The food grade sugar probes included in the test drink were $1 \mathrm{~g}$ lactulose (Centrafarm, Etten-Leur, The Netherlands), $1 \mathrm{~g}$ sucralose (Brenntag, Sittard, The Netherlands), 1 g erythritol (Danisco, Copenhagen, Denmark), 1 g sucrose (Van Gilse, Dinteloord, The Netherlands), and $0.5 \mathrm{~g}$ L-rhamnose (Danisco) dissolved in $150 \mathrm{~mL}$ tap water. GI permeability was assessed by determination of the $0-2 \mathrm{~h}$ urinary excretion of these orally ingested sugar probes using a combined high performance liquid chromatography (HPLC, Model PU1980 pump, Jasco Benelux, Maarsen, The Netherlands) and mass spectrometry (Model LTQXL, Thermo Electron, Breda, The Netherlands) approach (34). Gastroduodenal permeability was assessed by calculation of the $0-2 \mathrm{~h}$ urinary ratio of sucrose $(342 \mathrm{D})$ and L-rhamnose (164 $\mathrm{D})$, whereas the urinary $0-2 \mathrm{~h}$ ratio of lactulose $(342 \mathrm{D})$ and L-rhamnose, the $\mathrm{L} / \mathrm{R}$ ratio, was computed to assess small intestinal permeability.

\section{Statistical analysis}

Statistical analysis was performed using GraphPad Prism (Version 5.00, GraphPad Software for Windows, San Diego, CA). Normality of all data was verified by the Kolmogorov-Smirnov test. 
All normally distributed data are presented as mean \pm standard error of the mean (SEM), not normally distributed data as median and range. Continuous data were analyzed using two-way analysis of variance with Bonferroni post-hoc test for multiple comparisons. Correlations were determined by calculating the Spearman correlation coefficient $\left(\mathrm{R}_{\mathrm{S}}\right)$. Linear regression was used to visualize the correlation. Correlations between small intestinal injury and small intestinal permeability were computed using indvidually normalized I-FABP levels and $P<0.05$ was deemed statistically significant.

\section{RESULTS}

\section{Ibuprofen aggravates exercise-induced intestinal injury}

Plasma I-FABP levels were determined to assess loss of cellular integrity within the small intestine. In line with our previous study (33), plasma I-FABP levels gradually increased during cycling at $70 \%$ of $\mathrm{W}_{\max }$ from baseline $295 \pm 46 \mathrm{pg} / \mathrm{mL}$ to mean peak levels of $474 \pm 74 \mathrm{pg} / \mathrm{mL}$ immediately post-exercise $(P<0.05$; Figure $1 A)$. Interestingly, cycling with ibuprofen resulted in even higher levels of circulating I-FABP $(P<0.0001$; Figure $1 A)$. In the latter situation, peak IFABP levels of $875 \pm 137 \mathrm{pg} / \mathrm{mL}$ were observed immediately post-exercise, being a significant increase from baseline $(328 \pm 32 \mathrm{pg} / \mathrm{mL} ; P<0.05$; Figure $1 A)$. These peak I-FABP levels were significantly higher than I-FABP levels after cycling without ibuprofen $(875 \pm 137$ vs $474 \pm 74 \mathrm{pg} / \mathrm{mL}$, $P<0.05$; Figure $1 A, C)$.

Ibuprofen consumption also increased levels of small intestinal injury at rest $(P<0.05$; Figure $1 B, C)$. Two out of nine participants reported minor abdominal complaints during the study, including epigastric pain, flatulence and belching after ibuprofen administration. One individual (individual A) reported these symptoms only after ibuprofen administration at rest, and his symptoms were not accompanied by high plasma I-FABP levels ( $259 \mathrm{vs} 273 \mathrm{pg} / \mathrm{mL}$ with and without ibuprofen at rest, respectively). The other, individual B, reported symptoms on both test days after ibuprofen administration and had elevated plasma I-FABP levels (579 vs $495 \mathrm{pg} / \mathrm{mL}$ with and without ibuprofen at rest, and post-exercise levels of $1493 \mathrm{vs} 865 \mathrm{pg} / \mathrm{mL}$ for cycling with and without ibuprofen, respectively). Additionally, the I-FABP levels of participant B were considerably higher than the mean I-FABP levels of the total group depicted in Figure 1A,C. 
A
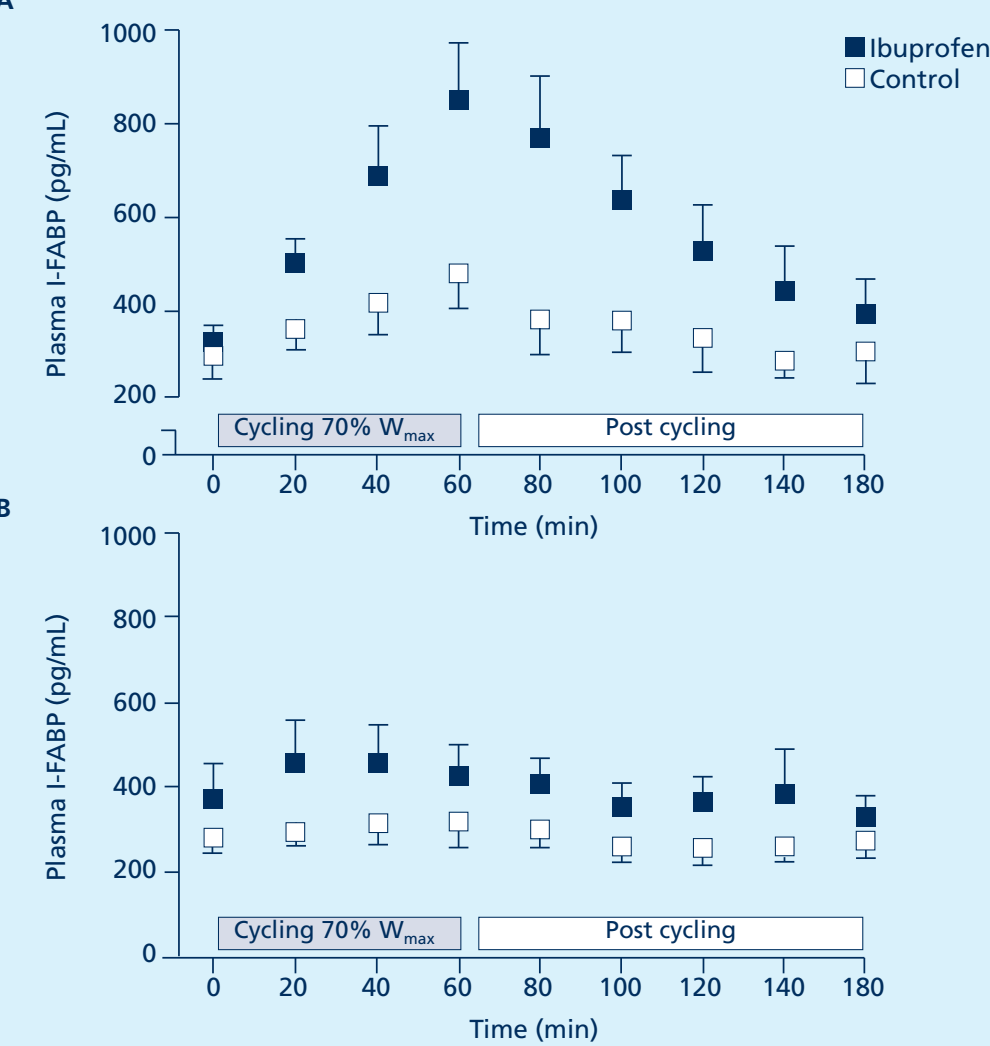

C

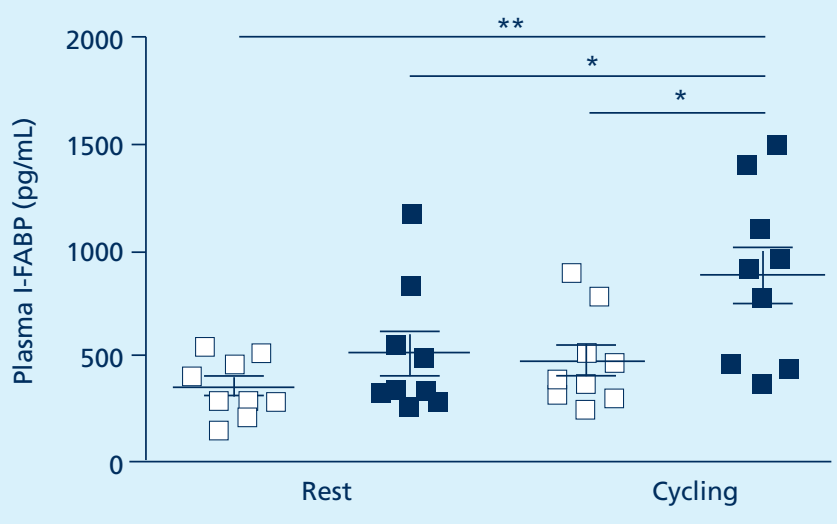

Figure 1: Ibuprofen significantly increases plasma I-FABP levels in rest and during cycling

A) Plasma I-FABP levels in athletes during and after cycling with prior intake of ibuprofen compared to cycling without ibuprofen $(P<0.0001)$. Squares represent mean data, with the SEM as black lines. B) Plasma I-FABP levels after intake of ibuprofen compared to control (i.e. no ibuprofen) in athletes at rest $(P=0.0003)$. Squares represent mean data, with the SEM as black lines. C) Peak plasma I-FABP levels in healthy athletes during rest and cycling conditions with or without ibuprofen. Squares represent individual data, with the mean \pm SEM depicted as black lines $\left({ }^{\star} P<0.05,{ }^{\star}{ }^{\star} P \leq 0.001\right)$. 
A

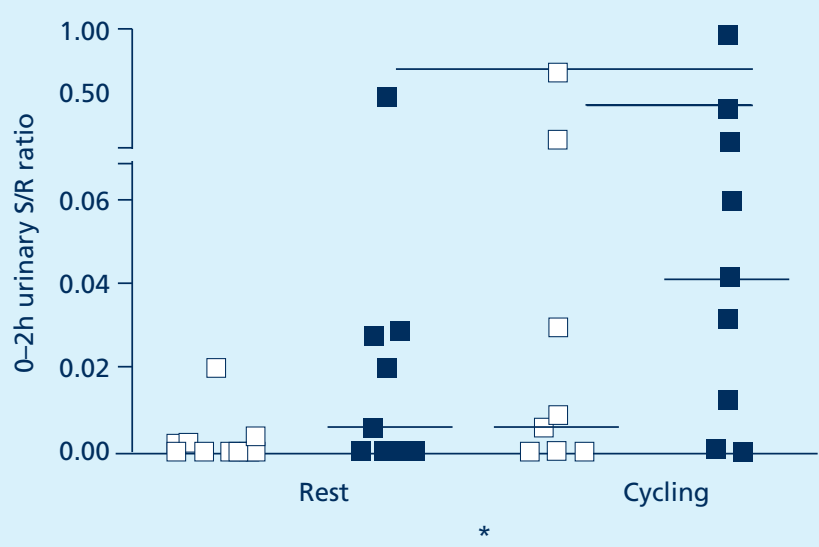

B

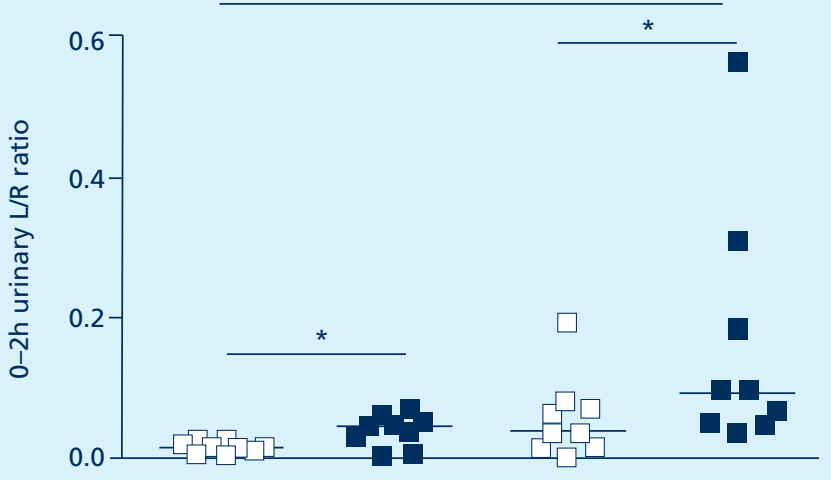

C

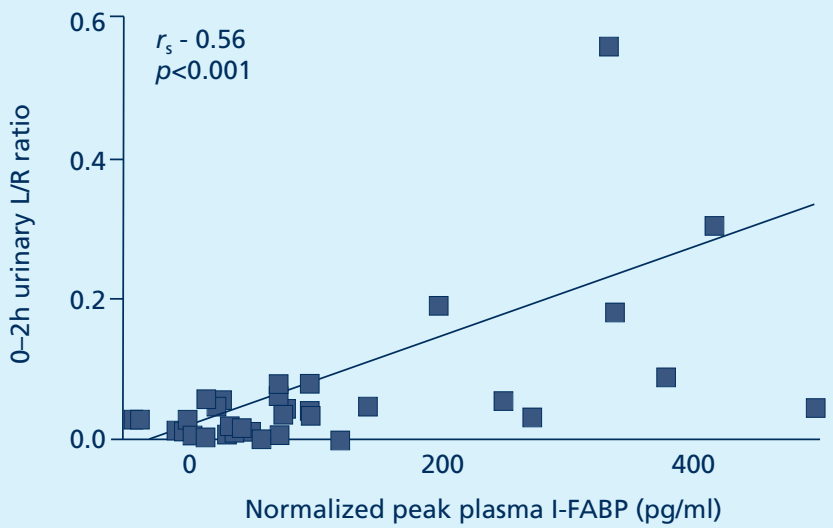

Figure 2: Ibuprofen increased 0-2h urinary sucrose/rhamnose (S/R) and lactulose/rhamnose (L/R) ratios A) $0-2 \mathrm{~h}$ urinary $\mathrm{S} / \mathrm{R}$ ratios in athletes after cycling with ibuprofen compared to rest without ibuprofen $(P=0.06)$. Squares represent individual data, with the median depicted as horizontal line. B) $0-2 \mathrm{~h}$ urinary $\mathrm{L} / \mathrm{R}$ ratios in athletes after cycling with ibuprofen compared to cycling and rest without ibuprofen. Squares represent individual data, with the median depicted as horizontal line $\left({ }^{\star} P<0.05\right)$. C) Correlation of $0-2 \mathrm{~h}$ urinary $\mathrm{L} / \mathrm{R}$ ratios with normalized plasma I-FABP levels. Squares represent individual data. 


\section{Ibuprofen prior to exercise increases gastroduodenal and small intestinal permeability}

Upper GI permeability increases after cycling with ibuprofen (0-2h S/R ratio 0.041(0.000-1.000) vs $0.000(0.000-0.020)$ compared with rest; $P=0.06$; Figure $2 A)$. Urinary $S / R$ ratios showed a weak but statistically significant correlation with peak plasma I-FABP levels $\left(R_{S}=0.49, P<0.005\right)$.

Consistent with gastroduodenal permeability, small intestinal permeability increased after cycling with ibuprofen in $0-2 \mathrm{~h}$ urinary $\mathrm{L} / \mathrm{R}$ ratio to $0.08(0.04-0.56)$ compared with $0.03(0.00$ 0.20 ) for cycling without ibuprofen. In addition, the $0-2 \mathrm{~h}$ urinary $\mathrm{L} / \mathrm{R}$ ration increased at rest with ibuprofen use to 0.05(0.01-0.07) compared with 0.01(0.01-0.04) without ibuprofen $(P<0.05$; Figure $2 B$ ). Interestingly, levels of intestinal injury correlated significantly with small intestinal permeability.

Peak plasma I-FABP levels of each test day correlated with $0-2 \mathrm{~h}$ urinary $\mathrm{L} / \mathrm{R}$ ratios $\left(\mathrm{R}_{\mathrm{S}}=\right.$ $0.56, P<0.001 ;$ Figure $2 C$ ). In line, total shedding of I-FABP from injured enterocytes, reflected by the Area Under the Curve (AUC) of plasma I-FABP levels, correlated with the 0-2h urinary $\mathrm{L} / \mathrm{R}$ ratios $\left(\mathrm{R}_{\mathrm{S}}=0.48, P<0.05\right.$; data not shown). Gastroduodenal and small intestinal permeability outcomes were not significantly different between symptomatic and asymptomatic participants, but individual A did show relatively high permeability ratios after ibuprofen consumption. In rest, the $0-2 \mathrm{~h} \mathrm{~S} / \mathrm{R}$ ratio of individual A was 0.475 , and 0.398 after cycling. The $0-2 \mathrm{~h} \mathrm{~L} / \mathrm{R}$ ratio of this individual was 0.058 in rest and 0.564 after cycling.

In summary, both gastroduodenal and small intestinal permeability increased after cycling following ibuprofen intake. Additionally, a clear relation was found between exercise-induced intestinal injury and small intestinal permeability after exercise. 


\section{DISCUSSION}

NSAID consumption is common among athletes. Alarmingly, its prevalence among athletes has been reported to reach $90 \%$ in specific sports $(16,29,32)$. Especially endurance athletes are well acquainted with the occurrence of GI problems during or after exercise (26,31). However, these athletes have limited awareness of the potentially hazardous effects of NSAIDs on the GI tract and their potential to induce GI discomfort (16). This study demonstrates that the use of ibuprofen prior to exhaustive exercise exacerbates exercise-induced small intestinal injury resulting in the loss of gut barrier function in healthy men.

Small intestinal injury as assessed by plasma I-FABP levels developed within one hour of cycling at $70 \%$ of $\mathrm{W}_{\max }$ and gradually returned to baseline levels during 60 minutes of subsequent post-exercise recovery. These data corroborated the findings of our previous study, in which we demonstrated that the exercise-induced intestinal compromise is at least partly caused by the rapid onset of splanchnic hypoperfusion (33). The latter is a result of the redistribution of splanchnic blood flow that occurs to secure adequate perfusion of muscles, skin, heart and lungs $(24,27)$. Hypoperfusion decreases the supply of oxygen and nutrients to the gut, leading to injury of the enterocytes lining the small intestinal villi $(5,6)$, as reflected by leakage of I-FABP from the affected enterocytes into the circulation (10). In line, injury of the GI tract after exercise has also been established using endoscopy, showing mucosal inflammation and erosive mucosal lesions in athletes after endurance running $(8,23)$.

The exact mechanisms by which NSAID consumption leads to GI damage and increased risk of upper GI complications such as mucosal lesions and perforation $(11,12,14)$ have not yet been fully elucidated. One of the major pathways considered to be involved is the inhibition of COX isotypes 1 and 2, resulting in local inflammation and vascular dysregulation, ultimately reducing perfusion and promoting mucosal integrity loss within the splanchnic area $(9,12,30)$. In addition, interference of NSAIDs with the production of NO due to prostaglandin-mediated microvascular dysfunction may further reduce mucosal blood flow (20). This NSAID-induced reduction of the splanchnic blood flow may deteriorate the exercise-induced state of splanchnic hypoperfusion, putting athletes at risk for serious GI compromise. Endurance athletes have been observed to have significant GI injury after endurance running without using NSAIDs $(8,23)$, and the combination of exercise and NSAIDs may aggravate this intestinal injury. Moreover, the negative effects of NSAIDs may not be restricted to the GI tract. Recent studies have demonstrated an increased risk of cardiovascular adverse events associated with the use of NSAIDs $(7,13,17,22)$.

In the present study, high levels of plasma I-FABP were observed in athletes after cycling, strongly suggesting intestinal injury. This intestinal damage was significantly more pronounced after administration of two $(400 \mathrm{mg}$ ) oral doses of the over-the-counter drug ibuprofen than after cycling without ibuprofen. Furthermore, ibuprofen intake induced abdominal discomfort in 2 out of the 9 athletes. The recommended maximum daily dose for oral ibuprofen intake varies from $1.2 \mathrm{~g}$ for 
non-prescription use to $2.4 \mathrm{~g}$ for prescribed oral administration (28). Our results clearly demonstrate that a considerably lower dose of ibuprofen prior to strenuous exercise aggravates exercise-induced small intestinal injury, leading to intestinal compromise. Since endurance athletes often use higher doses of NSAIDs and exercise for more than 1 hour, it may be expected that the intestinal compromise is more pronounced in daily practice. Although in the current study, the NSAID-induced small intestinal injury is reversible within 2 hours, long-term use of NSAIDs may prolong GI compromise, increasing the risk of GI complications, and potentially affecting performance and recovery.

Another key issue in the pathophysiology of NSAID-induced intestinal compromise is the development of barrier integrity loss and subsequent translocation of bacteria and permeation of harmful digestive enzymes $(4,21)$. These processes contribute to the local inflammatory response via activation of Toll-like receptor 4 and MyD88 (36). In the current study, loss of gut barrier function was demonstrated by an increase in urinary sugar permeability ratios. The compounding effect of NSAID consumption and heavy physical exercise led to increased gastroduodenal and small intestinal permeability, reflected by elevated urinary $S / R$ and $L / R$ ratios. The strong correlation that was found between cellular injury and the loss of barrier function in the small intestine emphasizes the extent of the intestinal compromise in the current study. Additionally, these data lead to the question whether the exercise-induced intestinal injury may also negatively affect the digestive and absorptive function of the GI system, thereby impeding postexercise recovery. In case of extensive intestinal damage, both the intestinal barrier function and the absorptive capacity of the GI tract may be compromised, yet future studies are warranted.

A limitation of the current study may be that we did not perform measurements to assess hydration status or renal function that may potentially affect plasma and urinary markers of intestinal compromise. GI permeability analysis in the current study was based on the urinary excretion of large and small sugar permeability probes, but because these probes are equally affected by factors as intestinal transit and renal function (3), alterations in renal function could not have influenced permeability analysis. Furthermore, all 15 athletes participating in our previous study had normal hydration status and no detectable kidney damage after completion of the same exercise protocol as applied in the current study (33). These data strongly suggest normal renal function in healthy athletes after 1 hour of cycling in our experimental setting. Endurance athletes exercising in a hot environment are more prone to become dehydrated, thereby potentially compromising renal function and clearance of NSAIDs.

In conclusion, the current study shows that in healthy endurance athletes, NSAID consumption can aggravate exercise-induced small intestinal injury and induces loss of gut barrier function. Although the ergogenic properties of NSAIDs remain questionable, evidence is provided to show that NSAIDs consumption is not harmless and may give rise to abdominal distress $(11,12,14)$. We consider it of utmost importance to increase the awareness of athletes and trainers toward the potential negative effects of NSAIDs, and recommend that the use of NSAIDs in the absence of a clear medical indication should be discouraged. 


\section{REFERENCES}

1. Alaranta A, Alaranta H, Heliovaara M, Airaksinen M, Helenius I. Ample use of physician-prescribed medications in Finnish elite athletes. Int F Sports Med. 2006;27(11):919-25.

2. Allison MC, Howatson AG, Torrance CJ, Lee FD, Russell RI. Gastrointestinal damage associated with the use of nonsteroidal antiinflammatory drugs. N Engl J Med. 1992;327(11):749-54.

3. Bjarnason I, MacPherson A, Hollander D. Intestinal permeability: an overview. Gastroenterology. 1995;108(5):1566-81.

4. Bjarnason I, Takeuchi K. Intestinal permeability in the pathogenesis of NSAID-induced enteropathy. $\mathcal{F}$ Gastroenterol. 2009;44 Suppl 19:23-9.

5. Blikslager AT. Life in the gut without oxygen: adaptive mechanisms and inflammatory bowel disease. Gastroenterology. 2008;134(1):346-8.

6. Blikslager AT MA, Gookin JL, Jones SL, Odle J. Restoration of barrier function in injured intestinal mucosa. Physiol Review. 2007;87 (2):545-64.

7. Cheetham TC, Graham DJ, Campen D, et al. Myocardial Infarction and Its Association with the Use of Nonselective NSAIDs: A Nested Case-Control and Time-to-Event Analysis. Perm f. 2008;12(1):16-22.

8. Choi SC, Choi SJ, Kim JA, et al. The role of gastrointestinal endoscopy in long-distance runners with gastrointestinal symptoms. Eur F Gastroenterol Hepatol. 2001;13(9):1089-94.

9. Crofford LJ. COX-1 and COX-2 tissue expression: implications and predictions. F Rheumatol Suppl. 1997;49:159.

10. Derikx JP, Matthijsen RA, de Bruine AP, et al. Rapid reversal of human intestinal ischemia-reperfusion induced damage by shedding of injured enterocytes and reepithelialisation. PLoS ONE. 2008;3(10):e3428.

11. Gabriel SE, Jaakkimainen L, Bombardier C. Risk for serious gastrointestinal complications related to use of nonsteroidal anti-inflammatory drugs. A meta-analysis. Ann Intern Med. 1991;115(10):787-96.

12. Garcia Rodriguez LA, Barreales Tolosa L. Risk of upper gastrointestinal complications among users of traditional NSAIDs and COXIBs in the general population. Gastroenterology. 2007;132(2):498-506.

13. Garcia Rodriguez LA, Gonzalez-Perez A, Bueno H, Hwa J. NSAID use selectively increases the risk of nonfatal myocardial infarction: a systematic review of randomised trials and observational studies. PLoS One. $2011 ; 6(2):$ e16780.

14. Garcia Rodriguez LA, Jick H. Risk of upper gastrointestinal bleeding and perforation associated with individual non-steroidal anti-inflammatory drugs. Lancet. 1994;343(8900):769-72.

15. Garcin M, Mille-Hamard L, Billat V, Imbenotte M, Humbert L, Lhermitte Z. Use of acetaminophen in young subelite athletes. I Sports Med Phys Fitness. 2005;45(4):604-7.

16. Gorski T, Cadore EL, Pinto SS, et al. Use of NSAIDs in triathletes: prevalence, level of awareness and reasons for use. Br F Sports Med. 2011;45(2):85-90.

17. Graham DJ, Campen D, Hui R, et al. Risk of acute myocardial infarction and sudden cardiac death in patients treated with cyclo-oxygenase 2 selective and non-selective non-steroidal anti-inflammatory drugs: nested casecontrol study. Lancet. 2005;365(9458):475-81.

18. Kuipers H, Verstappen FT, Keizer HA, Geurten P, van Kranenburg G. Variability of aerobic performance in the laboratory and its physiologic correlates. Int J Sports Med. 1985;6(4):197-201.

19. Lieberman JM, Sacchettini J, Marks C, Marks WH. Human intestinal fatty acid binding protein: report of an assay with studies in normal volunteers and intestinal ischemia. Surgery. 1997;121(3):335-42.

20. Martin MJ, Jimenez MD, Motilva V. New issues about nitric oxide and its effects on the gastrointestinal tract. Curr Pharm Des. 2001;7(10):881-908.

21. Matsui H, Shimokawa O, Kaneko T, Nagano Y, Rai K, Hyodo I. The pathophysiology of non-steroidal antiinflammatory drug (NSAID)-induced mucosal injuries in stomach and small intestine. $\mathcal{F}$ Clin Biochem Nutr. 2011;48(2):107-11.

22. Ng SC, Chan FK. NSAID-induced gastrointestinal and cardiovascular injury. Curr Opin Gastroenterol. 2010;26(6):611-7.

23. Oktedalen O, Lunde OC, Opstad PK, Aabakken L, Kvernebo K. Changes in the gastrointestinal mucosa after long-distance running. Scand f Gastroenterol. 1992;27(4):270-4.

24. Otte JA, Oostveen E, Geelkerken RH, Groeneveld AB, Kolkman JJ. Exercise induces gastric ischemia in healthy volunteers: a tonometry study. F Appl Physiol. 2001;91(2):866-71.

25. Pelsers MM, Hermens WT, Glatz JF. Fatty acid-binding proteins as plasma markers of tissue injury. Clin Chim Acta. 2005;352(1-2):15-35.

26. Peters HP, Bos M, Seebregts L, et al. Gastrointestinal symptoms in long-distance runners, cyclists, and triathletes: prevalence, medication, and etiology. Am f Gastroenterol. 1999;94(6):1570-81.

27. Qamar MI, Read AE. Effects of exercise on mesenteric blood flow in man. Gut. 1987;28(5):583-7. 
28. Rainsford KD. Ibuprofen: pharmacology, efficacy and safety. Inflammopharmacology. 2009;17(6):275-342.

29. Taioli E. Use of permitted drugs in Italian professional soccer players. Br J Sports Med. 2007;41(7):439-41.

30. Takeuchi K, Tanaka A, Kato S, Amagase K, Satoh H. Roles of COX inhibition in pathogenesis of NSAIDinduced small intestinal damage. Clin Chim Acta. 2010;411(7-8):459-66.

31. ter Steege RW, Van der Palen J, Kolkman JJ. Prevalence of gastrointestinal complaints in runners competing in a long-distance run: an internet-based observational study in 1281 subjects. Scand $\mathcal{f}$ Gastroenterol. 2008;43(12):1477-82.

32. Thuyne WV, Delbeke FT. Declared use of medication in sports. Clin F Sport Med. 2008;18(2):143-7.

33. van Wijck K, Lenaerts K, van Loon LJ, Peters WH, Buurman WA, Dejong CH. Exercise-induced splanchnic hypoperfusion results in gut dysfunction in healthy men. PLoS One. 2011;6(7):e22366.

34. van Wijck K, van Eijk HM, Buurman WA, Dejong CH, Lenaerts K. Novel analytical approach to a multi-sugar whole gut permeability assay. F Chromatogr B Analyt Technol Biomed Life Sci. 2011;879(26):2794-801.

35. Wallace JL. Prostaglandins, NSAIDs, and gastric mucosal protection: why doesn't the stomach digest itself? Physiol Rev. 2008;88(4):1547-65.

36. Watanabe T, Higuchi K, Kobata A, et al. Non-steroidal anti-inflammatory drug-induced small intestinal damage is Toll-like receptor 4 dependent. Gut. 2008;57(2):181-7. 


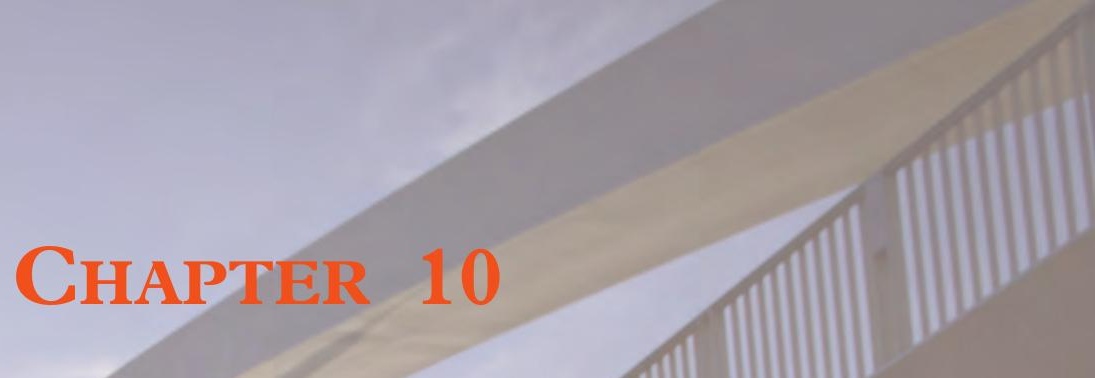

Oral L-citrulline supplementation improves splanchnic perfusion and reduces intestinal injury during strenuous exercise in healthy men
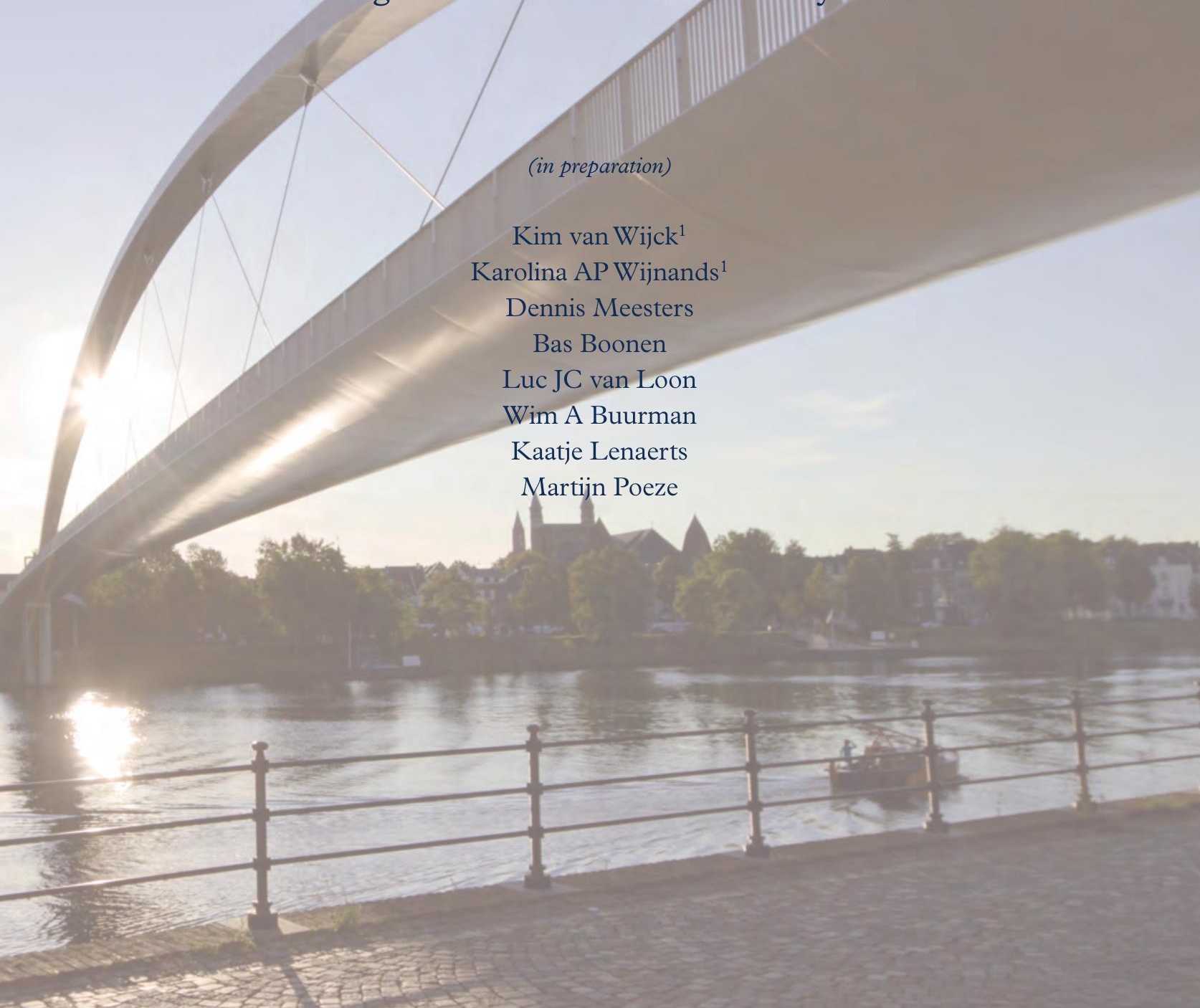


\section{ABSTRACT}

Splanchnic hypoperfusion is a physiological phenomenon during strenuous exercise. It has been associated with gastrointestinal symptoms, intestinal injury, and increased intestinal permeability in healthy athletes. We hypothesize that L-citrulline is able to improve splanchnic perfusion and decrease intestinal injury by enhancing the availability of arginine, the direct precursor of vasodilator nitric oxide. The aim of this study was to determine the effect of oral L-citrulline supplementation on splanchnic perfusion, intestinal injury and GI barrier function during and after exercise.

In this randomized, double-blinded crossover study, ten men cycled for 60 minutes at $70 \%$ of maximum workload after pre-exercise oral supplementation with L-citrulline (10 gram) or placebo (L-alanine) 30 minutes prior to exercise. Splanchnic hypoperfusion was assessed using gastric air tonometry. Sublingual microcirculation was evaluated by sidestream dark-field imaging. Blood, sampled every 10 minutes, was analyzed for amino acid levels and the enterocyte damage parameter intestinal fatty-acid binding protein (I-FABP). Changes in intestinal permeability were assessed using sugar probes.

Oral L-citrulline supplementation enhanced plasma citrulline $(1840.3 \pm 142.3 \mu \mathrm{M})$ and plasma arginine levels $(238.5 \pm 9.1 \mu \mathrm{M})$ during exercise compared to placebo $(45.7 \pm 4.8 \mu \mathrm{M}$ and 101.5 $\pm 6.1 \mu \mathrm{M}$, respectively), resulting in increased arginine availability following L-citrulline. L-citrulline supplementation preserved splanchnic circulation, reflected by stable gap $\mathrm{g}_{\mathrm{a}-\mathrm{a}} \mathrm{pCO}_{2}$ levels $(-1.70 \pm 0.19$ to $-1.45 \pm 0.63 \mathrm{kPa})$, and an increased number of perfused small sublingual vessels ( $7.8 \pm 6.0$ vs. $-2.0 \pm 2.4$ following placebo), while exercise-induced hypoperfusion was observed with placebo. Plasma I-FABP levels were attenuated during exercise following L-citrulline supplementation compared to placebo $\left(\mathrm{AUC}_{0-60 \mathrm{~min}}-185 \pm 506\right.$ vs. $\left.1318 \pm 553 ; P<0.01\right)$. No significant differences were observed for intestinal permeability.

In conclusion, pre-exercise oral L-citrulline supplementation preserves splanchnic perfusion during exercise compared to placebo in athletes, probably via enhanced arginine availability. Interestingly, oral L-citrulline also attenuated hypoperfusion-induced intestinal injury during exercise. These results suggest that oral L-citrulline supplementation is a promising therapy to combat splanchnic hypoperfusion-induced intestinal integrity loss. 


\section{INTRODUCTION}

Gastrointestinal (GI) symptoms are common during strenuous physical exercise, and range from mild nausea to 'angine abdominale' and hemorrhagic stool $(40,49)$. Incidence rates of gastrointestinal symptoms during strenuous exercise vary from 25 to $70 \%$, depending on exercise intensity and duration $(40,49)$. While the etiology of GI symptoms during exercise is thought to be multifactorial, decreased splanchnic perfusion has been postulated as a key mechanism $(15,36$, $48,49)$. During strenuous physical exercise, blood is redistributed away from the splanchnic area towards active muscles, the cardiopulmonary system and skin $(7,44)$, thereby strongly reducing splanchnic blood flow $(38,54)$. We have recently demonstrated that splanchnic hypoperfusion is associated with small intestinal cellular injury and loss of gut barrier function in healthy individuals (54). Intestinal cellular and barrier integrity loss are undesirable phenomena especially for athletes, since they may lead to abdominal distress, compromised food uptake and may interfere with early recovery (53). Disruption of the intestinal barrier may lead to more GI injury, since it exposes the submucosal tissue to bacteria, potentially harmful pancreatic juices and digestive products (4). The splanchnic microcirculatory blood flow is a potential target to prevent exercise-induced splanchnic compromise (26). One way to enhance the intestinal microcirculation is to increase the local availability of nitric oxide (NO), an important endogenous regulator of the microcirculation (32). NO is produced in the intestine via oxidation of L-arginine upon activation of endothelial nitric oxide synthetase (eNOS) in endothelial cells and inducible NOS (iNOS) during ischemia-induced intestinal damage (33). NO inhalation has been applied as therapeutic agent in experimental settings in patients with pulmonary arterial hypertension to induce local vasodilatation (21) and was found to attenuate lung ischemia-reperfusion injury in animal studies (20). However, since NO is a gas, it cannot easily be applied locally to other organs than the lungs. Therefore, many NO-donors have been studied to increase local NO levels to improve mucosal oxygenation. L-arginine was one of the first agents studied in this respect, and while its administration did increase plasma arginine levels and NO availability to some extent, the results were not unequivocally positive $(5,8,41,43)$. Oral supplementation of citrulline, a non-protein amino acid and an important precursor of arginine (50,60), has been shown to increase L-arginine availability for $\mathrm{NO}$ production to a greater extent than L-arginine supplementation itself $(11,45,46)$. The latter is explained by a combination of factors: (a) Lcitrulline is transported more efficiently from the lumen into the circulation than L-arginine; (b) a balanced, probably near null flux across the liver exists for L-citrulline, which means that L-citrulline hepatic uptake is counterbalanced by L-citrulline release $(11,50,51)$ and citrulline released from the gut enters the systemic circulation 'untouched'; and (c) L-citrulline is actively converted to L-arginine within endothelial cells so that L-arginine can be used for NO-production rather than to induce ureagenesis (61). Metabolisation of L-citrulline occurs in the kidneys, where it is converted via argininosuccinate synthetase (ASS) and argininosuccinate lyase (ASL) 
into L-arginine $(1,11)$. Ten gram of oral L-citrulline supplementation in healthy subjects has been shown to increase plasma citrulline and arginine levels and to improve muscle protein synthesis (22), without causing negative side effects such as diarrhea, GI discomfort and nausea, as seen after L-arginine supplementation (24). Therefore, L-citrulline seems to be a promising substrate to enhance the intestinal L-arginine availability $(34,59)$, thereby restoring the intestinal NO production in endothelial cells and increasing the intestinal microcirculatory perfusion (59). Based on these findings, we hypothesized that oral supplementation of 10 gram L-citrulline prior to strenuous exercise increases the arginine availability in healthy athletes; thereby improving splanchnic perfusion and reducing exercise-induced GI injury, and GI barrier loss. This was tested in a randomized, double-blinded, placebo-controlled crossover study.

\section{METHODS AND MATERIALS}

\section{Subjects' characteristics}

Ten healthy recreationally active men (age: $24.9 \pm 1.0$ years, body mass index: $21.1 \pm 0.4 \mathrm{~kg} / \mathrm{m}^{2}$ ) were selected to participate in the present study. Maximal workload capacity $\left(\mathrm{W}_{\max }\right)$, assessed prior to the experiments on a stationary cycle ergometer (Lode Excalibur, Groningen, the Netherlands) (29) was $5.0 \pm 0.1 \mathrm{~W} / \mathrm{kg}$, with maximum heart rate of $194 \pm 2 \mathrm{bpm}$. The selected volunteers all spent 3 to $15 \mathrm{~h}$ per wk performing endurance sports as part of their normal life style. The volunteers had no abdominal complaints during daily activities, had not taken any medication for at least one month prior to participation, had no history of GI disease, and had no history of abdominal surgery. Selected volunteers were informed about the nature and risks of the experiments, after which written consent was obtained at least $5 \mathrm{~d}$ prior to the experiments. This study was approved by the medical ethics committee of Maastricht University Medical Centre+ (MEC 10-3-064), and conducted in accordance with the Declaration of Helsinki (revised version, October 2008, Seoul).

Participants maintained normal activities of daily living, but refrained from strenuous physical activity for $2 \mathrm{~d}$ prior to each test day. Participants were not allowed to consume alcohol and artificial sweeteners the day prior to the experiments. Ranitidine (150 mg, GlaxoSmithKline, Zeist, the Netherlands) was ingested by the participants on the evening prior to each test day to inhibit gastric acid production and secretion. The latter is necessary to enable gastric tonometry measurements, since the presence of acid in the stomach during tonometry can buffer carbon dioxide molecules, thereby interfering with the outcome of the tonometry measurements (28). An additional $150 \mathrm{mg}$ of ranitidine was consumed on the morning of the test days, at 07:00, being 60 min before start of the experiments. 


\section{Study design and nutritional intervention}

In this double-blind randomized controlled crossover study, the participants were randomized for two different interventions, i.e. oral L-citrulline supplementation and placebo supplementation. All subjects performed the two test days in random order: (1) after oral intake of a $10 \mathrm{~g}$ bolus of L-citrulline dissolved in $125 \mathrm{~mL}$ tap water and (2) after intake of the $125 \mathrm{~mL}$ placebo drink, containing $20 \mathrm{~g}$ of L-alanine in tap water. Both L-citrulline and the placebo were tested safe for human oral consumption by Basic Pharma Group (Geleen, the Netherlands), and supplied as powders to be dissolved 30 minutes prior to intake by the participants. The test drinks were isonitrogenous and had no specific taste. No additives or sweeteners were used. Time between test days was at least $7 \mathrm{~d}$.

\section{Experiments and sampling}

All experiments were performed after an overnight fast. Upon arrival to the sports laboratory at 08:00 A.M., participants were instructed to collect a urinary sample. To enable collection of arterialized blood for analysis of arterial $\mathrm{pCO}_{2}$ levels, a catheter (22 Gauge, Braun, Melsungen, Germany) was inserted in a dorsal hand vein of the participant, and the hand was placed in a hot box set at $60^{\circ} \mathrm{C}(27)$. To measure gastric $\mathrm{pCO}_{2}$, an 8 French tonometrics catheter (Datex Ohmeda, Finland) was introduced via the nose into the stomach of the participant, and fixed to the nasal flares. Gastric $\mathrm{pCO}_{2}$ was measured at 10-minute intervals before, during, and after cycling using an automated capnograph (Tonocap TC-200, Datex Ohmeda, Finland). Arterialized blood samples were collected simultaneously with the tonometry measurements in heparin tubes and gastricarterialized $\mathrm{pCO}_{2}\left(\mathrm{p}_{\mathrm{g}-\mathrm{a}} \mathrm{pCO}_{2}\right.$ gap) was calculated. At each time point, a second blood sample was collected into pre-chilled ethylenediaminetetraacetic acid (EDTA) tubes (Vacucontainer, Becton Dickinson (BD), Helsingborg, Sweden) for analysis of plasma parameters. After calibration of the tonometry device, side-stream dark field (SDF) sublingual imaging was performed for baseline assessment of the microcirculation as described below. After baseline measurements, participants ingested the test drink they were assigned to for that day. 30 minutes after intake of the test drink, participants started cycling at a workload of $150 \mathrm{~W}$. After $3 \mathrm{~min}$, workload was increased to $70 \%$ of the individual's pre-assessed $\mathrm{W}_{\max }\left(70 \%\right.$ of $\mathrm{W}_{\max }$ was $\left.248.2 \pm 7.6 \mathrm{~W}\right)$. Subjects maintained pedal rates of at least $60 \mathrm{rpm}$, and workload was decreased by $25 \mathrm{~W}$ if participants were unable to maintain the $60 \mathrm{rpm}$. Participants consumed tap water ad libitum, with a minimum of $50 \mathrm{~mL}$ and a maximum of $150 \mathrm{~mL}$ every $10 \mathrm{~min}$. After 30 minutes of cycling, the test subjects ingested a 150 $\mathrm{mL}$ multi-sugar drink to enable whole gut permeability analysis, while cycling to complete the 60 minute exercise bout. Immediately post-exercise, SDF imaging was performed to measure sublingual microcirculation. Additional SDF imaging was performed at 30 minutes and 1 hour after cycling, while a second urinary sample was collected 90 minutes after cycling. All collected blood and urine samples were centrifuged as soon as possible after collection at $4^{\circ} \mathrm{C}$ at $2300 \mathrm{x} g$ for 15 min, and stored at $-80^{\circ} \mathrm{C}$ until analysis. Any GI complaints of the subjects during the test days 
were registered by the researchers, and subjects were contacted $2 \mathrm{~d}$ after the test day to assess the occurrence of GI complaints on the day following each test day.

\section{Assessment of circulating amino acids}

To evaluate whether the L-citrulline supplementation resulted in an enhanced arginine availability index, plasma amino acids concentrations were measured and the arginine availability index (AAI) was calculated as $(9,35)$

$$
\frac{\text { [arginine }]}{\text { [ornithine] }+ \text { [lysine }]}
$$

and is an index based on the cellular uptake of arginine, ornithine, and lysine by the same transport system, the $\mathrm{y}^{+}$transporter. Plasma amino acid analysis was performed after blood collection and centrifugation. A $100 \mu \mathrm{L}$ plasma aliquot was pipetted into a $1.5 \mathrm{~mL}$ Eppendorf tube that already contained $5.5 \mathrm{mg}$ solid sulfosalicylic acid (SSA), vortex-mixed immediately to deproteinize the plasma samples, snap frozen in liquid nitrogen and stored at $-80^{\circ} \mathrm{C}$ until analysis.

Before analysis, deproteinized plasma samples were thawed, vortex-mixed and centrifuged for $10 \mathrm{~min}$ at $50,000 \mathrm{xg}$ at $4^{\circ} \mathrm{C}$ in a Biofuge Stratos centrifuge (Heraeus, Haarlem, the Netherlands). Next, $5 \mu \mathrm{L}$ of the clear supernatant was diluted 100-fold in ice-cold water into a $1 \mathrm{~mL}$ WISPstyle vial (Waters, Etten-Leur, the Netherlands). Amino acid analysis was performed by HPLC after automated pre-column derivatization using ophthaldialdehyde (OPA) as described before (52). Briefly, at the start of each cycle, $5 \mu \mathrm{L}$ of the diluted sample, stored in the pre-chilled sample compartment of a WISP autosampler, was automatically mixed with $5 \mu \mathrm{L}$ of OPA reagent. The resulting OPA-amino acid derivatives were separated on a 150x4.6 mm (inner diameter) Allsphere ODS $23 \mu \mathrm{M}$ High-Performance Liquid Chromatography (HPLC) column (Grace, Breda, the Netherlands), using an acetonitrile gradient against an aqueous citric acid buffer $(25 \mathrm{mmol} / \mathrm{L}$, $\mathrm{pH}=6.8$ ) and detected by fluorescence ( $330 \mathrm{~nm}$ excitation, $440 \mathrm{~nm}$ emission).

\section{Assessment of microcirculation}

To evaluate the intestinal microcirculation during exercise, the sublingual microcirculation was measured to reflect with an SDF imager $(25,47)$. SDF imaging was used to discriminate between vessels of different diameter and to assess the proportion of perfused sublingual vessels. The SDF imager uses 530nm light absorbed by the hemoglobin in red blood cells which allows observation of these cells in the microcirculation (6). All imaging measurements were done by an experienced investigator. Using a camera magnification of $5 \mathrm{x}$, sharp real-time images of the sublingual microcirculation were obtained in a field of $1000 \times 750 \mathrm{~m}$. In total 20-sec continuous image sequences per time-point, each consisting of 200 images, were recorded at five sublingual sites according to the consensus of a round table conference $(6,14,57)$.Videos were analyzed by 2 independent researchers according to De Backer et al. using the Automated Vascular Analysis (AVA) software 3.0 (Microscan, Amsterdam, The Netherlands) (14). The total number of perfused small vessels and the total number of perfused vessels were measured. 


\section{Assessment of small intestinal injury}

To evaluate the extent of small intestinal injury during and after cycling in both situations, plasma concentrations of human Intestinal Fatty Acid Binding Protein (I-FABP) were determined by a researcher blinded for the specific test conditions. I-FABP is a small, 15-kD cytosolic protein that is present especially in mature enterocytes of the small intestine. Upon enterocyte injury, the protein rapidly diffuses through the interstitial space into the circulation, enabling its detection in plasma samples $(31,39)$. Plasma I-FABP levels were measured by an in-house developed enzyme-linked immunosorbent assay (ELISA): ELISA plates were coated with anti-I-FABP immunoglobulin $\mathrm{G}$ (IgG) overnight at $4^{\circ} \mathrm{C}$; free sites were blocked with $1 \%$ bovine serum albumin (BSA) in PBS. The samples and human recombinant I-FABP for standard calibration curves were incubated at room temperature and biotinylated anti-I-FABP IgG was added. After washing, horseradish peroxidase-streptavidin conjugate (Zymed Laboraties Inc., San Francisco, CA) in $0.1 \%$ BSA-PBS and 3,3,5,5-tetramethylbenzidine (Kirkegaard \& Perry Laboratories, Gaithersburg, MD) were added. The reaction was stopped and color intensity was measured with an ELISA reader at $450 \mathrm{~nm}$. The detection window of the I-FABP assay was 12.5 to 800 $\mathrm{pg} / \mathrm{mL}$.

\section{Assessment of GI permeability}

Permeability analysis was performed as a measure of GI barrier integrity, using a multi-sugar test drink as described previously $(55,56)$. The food grade sugar probes included in the test drink were $1 \mathrm{~g}$ lactulose (Centrafarm, Etten-Leur, the Netherlands), $1 \mathrm{~g}$ sucralose (Brenntag, Sittard, the Netherlands), 1 g erythritol (Danisco, Copenhagen, Denmark), 1 g sucrose (Van Gilse, Dinteloord, the Netherlands), and $0.5 \mathrm{~g} \mathrm{~L}$-rhamnose (Danisco) dissolved in $150 \mathrm{~mL}$ tap water. GI permeability was assessed by determination of urinary concentrations of these orally ingested sugar probes using a combined HPLC (Model PU-1980 pump, Jasco Benelux, Maarsen, the Netherlands) and mass spectrometry (Model LTQ-XL, Thermo Electron, Breda, the Netherlands) approach (55). Small intestinal permeability was reflected by the $0-2 \mathrm{~h}$ urinary excretion ratio lactulose (342 D) and L-rhamnose (164 D), the $\mathrm{L} / \mathrm{R}$ ratio.

\section{Statistical analysis}

Statistical analysis was performed using GraphPad Prism (Version 5.00, GraphPad Software for Windows, San Diego, CA). Normality of all data was verified by the Kolmogorov-Smirnov test. Data are presented as mean \pm standard error of the mean (SEM). Continuous data were analyzed using two-way analysis of variance with Bonferroni post-hoc test for multiple comparisons. For within group comparisons, either one-way analysis of variance with Bonferroni post-hoc test or Friedman with Dunn's post-hoc test were used depending on data normality. A $P<0.05$ was considered statistically significant. 


\section{RESULTS}

\section{Oral L-citrulline supplementation prior to exercise enhances the arginine availability index during exercise compared with placebo}

As expected, oral L-citrulline supplementation led to increased plasma citrulline concentrations (peak 1840.3 $\pm 142.3 \mu \mathrm{M}$ ) compared to baseline $(33.6 \pm 2.9 \mu \mathrm{M}$ ) and compared to placebo L-alanine (peak 45.7 $\pm 4.8 \mu \mathrm{M}$; Figure $1 \mathrm{~A}$ ). Plasma citrulline concentrations remained low throughout the studied period after placebo supplementation (Figure 1A), while L-alanine levels increased profoundly following placebo administration (peak 1639.1 $112.0 \mu \mathrm{M}$ versus peak 434.8 $\pm 25.5 \mu \mathrm{M}$ after L-citrulline; Figure 1B).

The increased citrulline concentrations after L-citrulline supplementation were accompanied by a significant increase in plasma arginine concentrations during exercise $(238.5 \pm 9.1 \mu \mathrm{M}$ directly after exercise compared to $81.8 \pm 3.4 \mu \mathrm{M}$ at baseline; Figure $1 C$ ), while supplementation with placebo only slightly increased plasma arginine concentrations $(101.5 \pm 6.1 \mu \mathrm{M}$ vs. $78.4 \pm 3.4$ $\mu \mathrm{M}$; Figure $1 C$ ). The increase in arginine concentrations following oral L-citrulline supplementation significantly enhanced the AAI (0.76 \pm 0.02 vs. baseline $0.38 \pm 0.02$; Figure $1 D)$, while placebo supplementation did not influence AAI (0.38 \pm 0.02 to $0.42 \pm 0.03$; Figure $1 D)$. Interestingly, both the plasma L-arginine levels and AAI remained elevated after oral L-citrulline supplementation during $1 \mathrm{~h}$ of exercise and at least until the end of our experiments, $1 \mathrm{~h}$ post-exercise (Figure $1 C$ and D). These observations indicate a prolonged effect of a single $10 \mathrm{~g}$ bolus of L-citrulline prior to exercise. No arginine deficiency was observed during exercise after either of the supplementation strategies.

\section{Oral citrulline supplementation prior to exercise maintains adequate splanchnic perfusion during exercise}

$\mathrm{P}_{\mathrm{g}-\mathrm{a}} \mathrm{pCO}_{2}$ gap levels, measured by gastric tonometry, reflect splanchnic perfusion. Tonometry data were obtained from 9 participants, due to inability to introduce the nasogastric catheter into the stomach of one of the participants. Strenuous exercise quickly resulted in a significant change in $\mathrm{p}_{\mathrm{g}-\mathrm{a}} \mathrm{pCO}_{2}$ gap levels during exercise after pre-exercise placebo supplementation $(-1.67 \pm 0.32$ to a peak of $-0.55 \pm 0.46 \mathrm{kPa}$ at 30 minutes of exercise), while no significant change from baseline was observed during exercise after oral L-citrulline supplementation $(-1.70 \pm 0.19$ to $-1.45 \pm 0.63 \mathrm{kPa}$; Figure $2 A$ ), pointing to preserved perfusion if L-citrulline was applied prior to exercise. The inter-individual differences in $\mathrm{p}_{\mathrm{g}-\mathrm{a}} \mathrm{pCO}_{2}$ gap levels were relatively high during exercise, reflected by relatively large SEMs.

Nonetheless, area under the curve (AUC) calculations of $\mathrm{p}_{\mathrm{g}-\mathrm{a}} \mathrm{pCO}_{2}$ gap during exercise revealed a trend $(P=0.10)$ towards a difference between $\mathrm{L}$-citrulline and L-alanine supplementation $(-3.3 \pm$ 20.1 vs. $40.1 \pm 20.6$, respectively; Figure $2 B$ ). After exercise, a trend towards an increase in $\mathrm{p}_{\mathrm{g}-\mathrm{a}} \mathrm{pCO}_{2}$ gap levels was seen after both supplementation strategies, but no statistical significance was reached. 

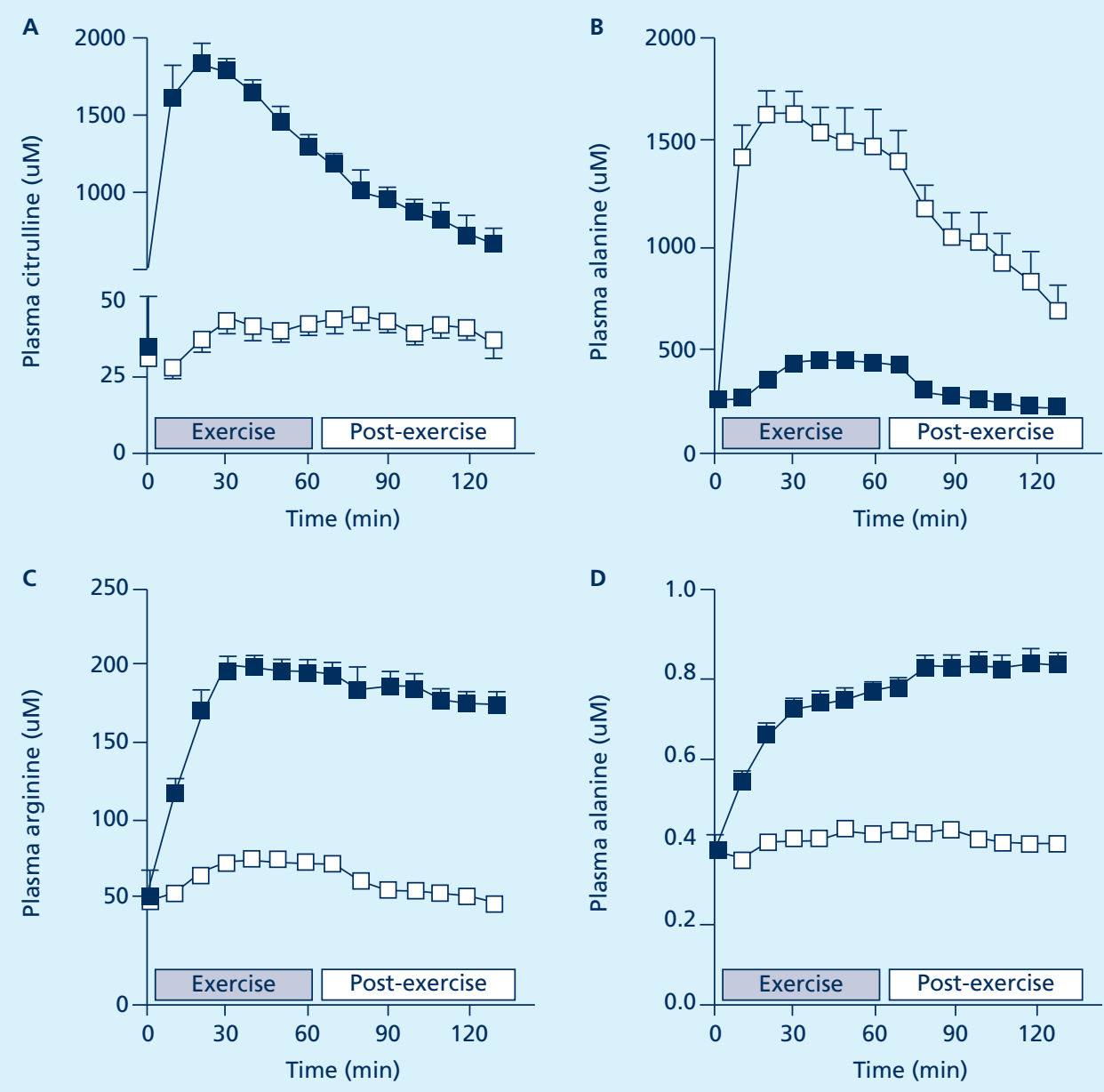

Placebo Citrulline

Figure 1: Mean ( $\pm S E M)$ plasma concentrations of L-citrulline (A), L-alanine (B), L-arginine (C) and L-arginine availability (D) after oral bolus of placebo or L-citrulline in men $(n=10)$ during and after exercise.

The data were analyzed with two-way repeated measures ANOVA. Citrulline: time effect, $P<0.0001$; treatment effect, $P<0.0001$; alanine: time effect, $P<0.0001$; treatment effect, $P<0.0001$; arginine: time effect, $P<0.0001$; treatment effect, $P<0.0001$, and arginine availability: time effect, $P<0.0001$; treatment effect, $P<0.0001$.

\section{Citrulline maintains sublingual vessel perfusion after exercise}

In line with the tonometry data, analysis of the sublingual microcirculation revealed that citrulline preserves the perfusion of the microvessels. After L-citrulline supplementation, the number of perfused small vessels under the tongue was $(P=0.06)$ higher immediately post-exercise and 30 minutes post-exercise than after L-alanine supplementation (Figure $3 \mathrm{~A}$ ), indicating a trend towards a positive change in perfusion of the small sublingual vessels in favor of L-citrulline 


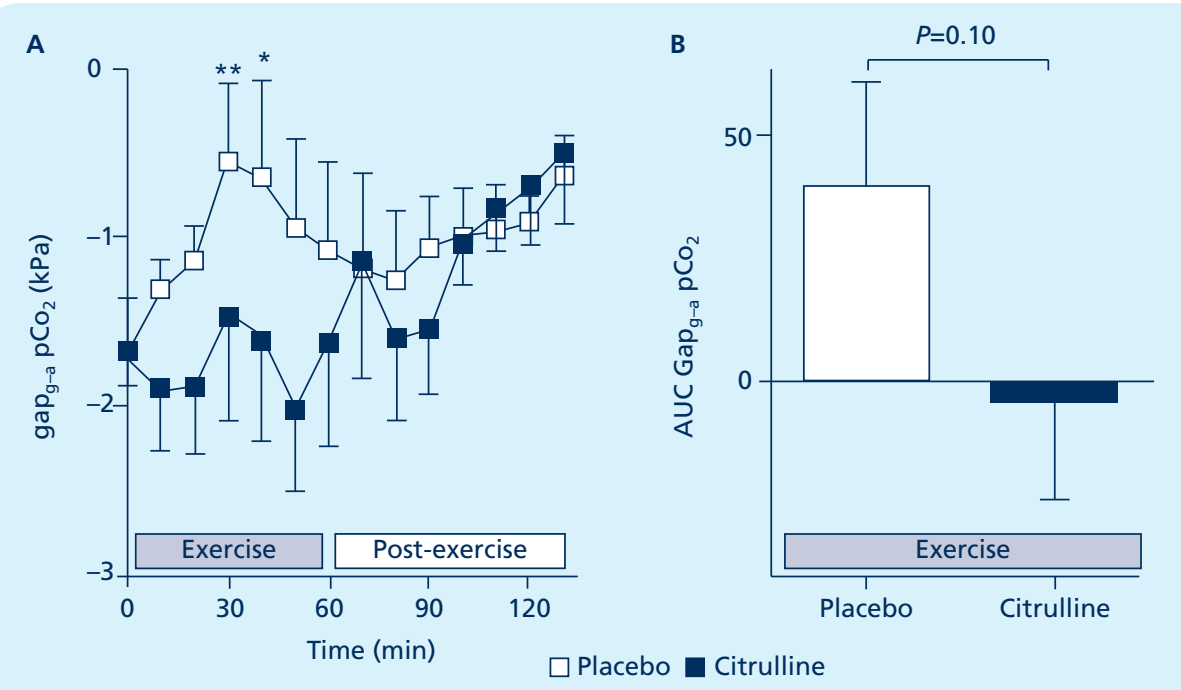

Figure 2: Mean and SEM of gap $p_{g-a} p C \mathrm{O}_{2}$ levels during and after exercise (A) and area under the curve (AUC) of $p_{g-a} p \mathrm{CO}_{2}$ gap levels during exercise $(B)$ in men $(n=10)$ after consumption of placebo or L-citrulline.

The data over time were analyzed with two-way repeated measures ANOVA. Time effect, $P<0.01$; treatment effect, $P=0.44$; asterisks indicate different from baseline $(0 \mathrm{~min}){ }^{\star} P<0.05,{ }^{\star \star} P<0.01$. AUC data over the first 60 minutes were analyzed with paired t-test.

(7.8 \pm 6.0 for L-citrulline vs. $-2.0 \pm 2.4$ following placebo; Figure 3B). Additionally, the total number of perfused vessels significantly improved post-exercise in case of L-citrulline supplementation prior to exercise, compared to a decrease in sublingual perfusion in case of placebo administration ( $50.7 \pm 2.9$ and $38.0 \pm 1.7$, respectively; Figure $3 C$ ). In line, the AUC calculations revealed a significant increase in total sublingual perfusion of vessels post-exercise in case of L-citrulline supplementation, compared to a decrease in perfusion if placebo was supplemented before exercise (12.0 \pm 6.1 vs. $-7.4 \pm 1.7$; Figure $3 D)$.

\section{Citrulline reduces exercise-induced small intestinal injury during exercise}

To assess the effect of oral L-citrulline supplementation on small intestinal epithelium, plasma I-FABP levels were determined every ten minutes before, during and after exercise. The increase in plasma I-FABP levels, depicted as percentage from baseline, indicating small intestinal enterocyte damage, reached significance after 1 hour of strenuous exercise in case of oral placebo supplementation prior to exercise, while the increase in plasma I-FABP levels was less pronounced after L-citrulline administration prior to exercise (Figure $4 A$ ). Post-exercise levels of I-FABP increased by approximately $72 \%$ above baseline after both citrulline and alanine, and gradually declined towards baseline levels in the post-exercise recovery period (Figure $4 \mathrm{~A}$ ). A marked difference of the effect of citrulline- and alanine-supplementation was observed especially during exercise. AUC calculations of the IFABP levels (as percentage from baseline) during 

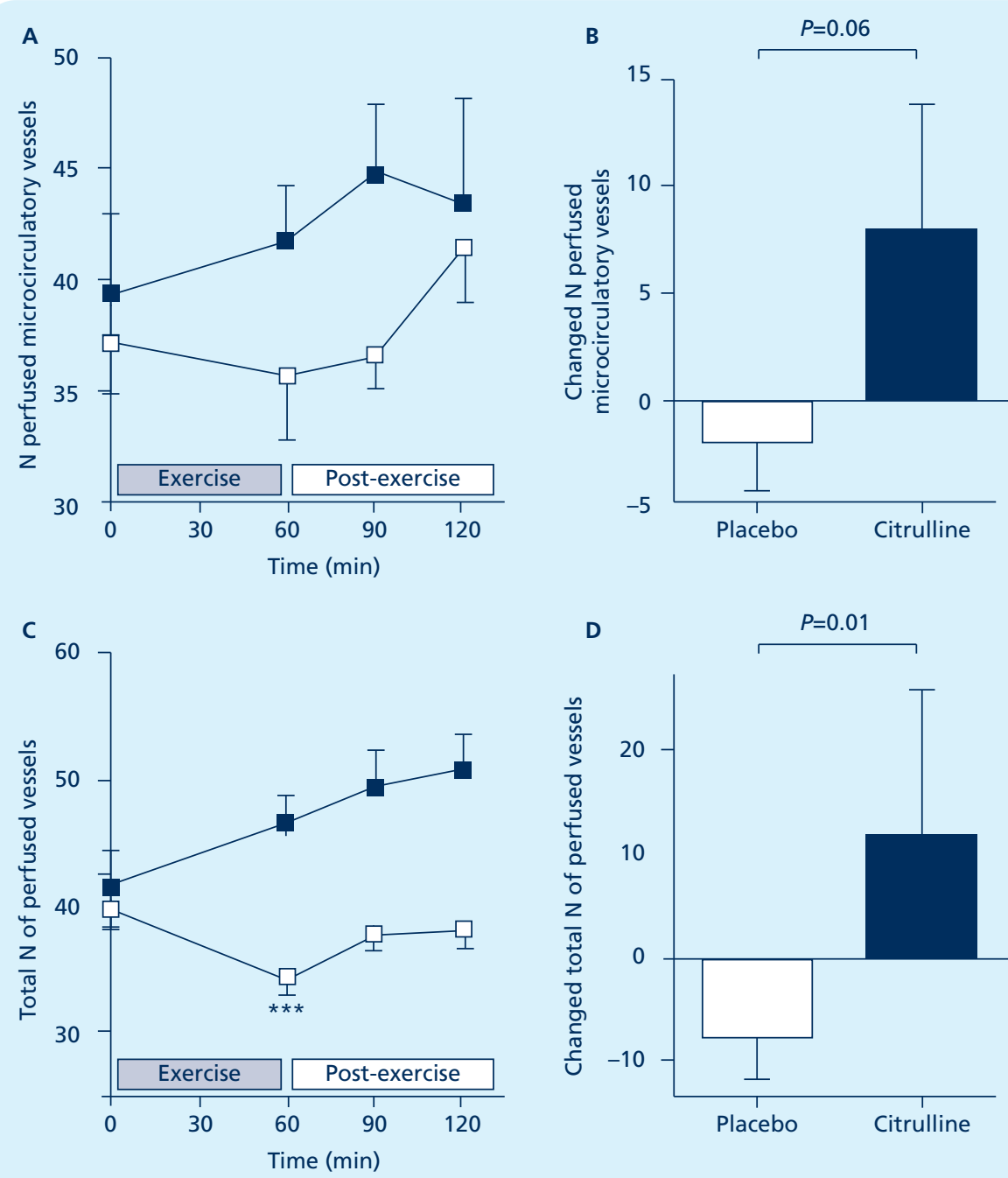

$\square$ Placebo $\square$ Citrulline

Figure 3: Total number of sublingual perfused microcirculatory vessels (mean $\pm S E M)(A)$, change in microcirculatory vessel number (B), total number of perfused sublingual vessels (C), change in total vessel number (D) during and after exercise in men $(n=10)$ after consumption of placebo or L-citrulline.

The data over time were analyzed with two-way repeated measures ANOVA. Microcirculatory vessel number until 30 min post-exercise: time effect, $P=0.37$; treatment effect, $P=0.09$. Total vessels number until 30 min post-exercise: time effect, $P<0.05$; treatment effect, $P<0.001$; asterisks indicate different from baseline $(\mathrm{t}=0 \mathrm{~min}){ }^{\star \star \star} P<0.001$. Changes in microcirculatory and total vessel number until $30 \mathrm{~min}$ post-exercise were analyzed with paired t-test. 


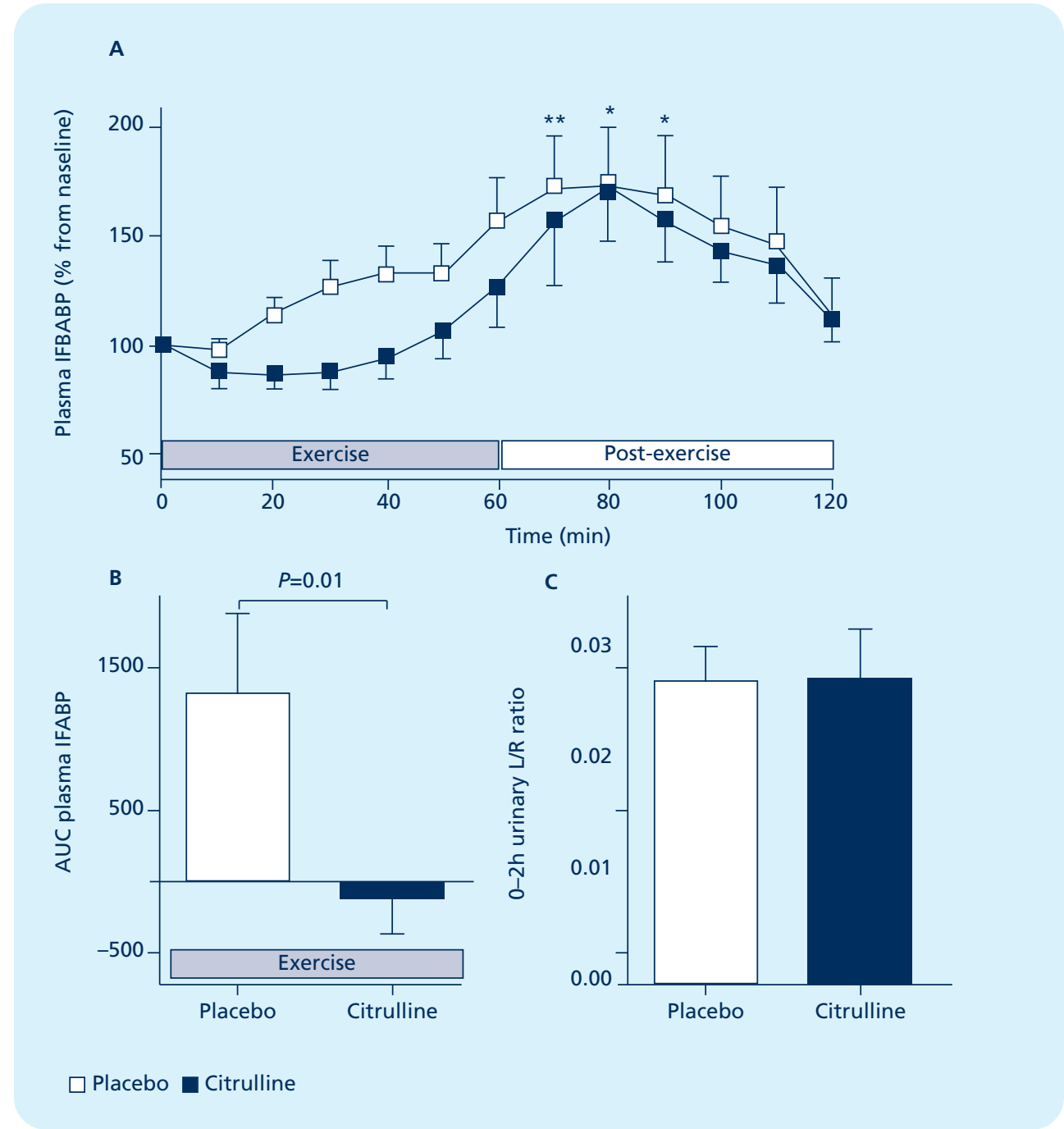

Figure 4: Mean ( $\pm S E M)$ percentage changes in plasma IFABP during and after exercise $(A)$, area under the curve (AUC) of percentage IFABP change during exercise (B), and L/R urinary excretion over 2-hour period $(C)$ in men $(n=10)$ after consumption of placebo or L-citrulline.

The data over time were analyzed with two-way repeated measures ANOVA. Time effect, $P<0.0001$; treatment effect, $P=0.07$; asterisks indicate different from baseline $(0 \mathrm{~min}){ }^{\star} P<0.05,{ }^{\star} P<0.01$. AUC data over the first 60 minutes and urinary sugar excretion were analyzed with paired t-test.

exercise revealed a significant difference $(P<0.01)$ between L-citrulline and L-alanine supplementation (-185 \pm 506 vs. $1318 \pm 553$, respectively; Figure $4 B$ ). Hence, oral L-citrulline supplementation was able to prevent a net increase in plasma I-FABP levels during exercise, compatible with diminished exercise-induced intestinal injury. 


\section{Exercise-induced GI permeability changes}

To analyze whether oral L-citrulline supplementation prior to exercise would maintain GI barrier function during exercise, permeability $\mathrm{L} / \mathrm{R}$ ratios were determined in urine collected in the $0-2 \mathrm{~h}$ post-exercise recovery period. Urinary $\mathrm{L} / \mathrm{R}$ ratio was $0.027 \pm 0.003$ after oral administration of L-alanine prior to exercise, compared to $0.024 \pm 0.002$ after administration of L-citrulline (Figure $4 C$ ). These data indicate that no significant difference was observed between the two nutritional interventions with respect to small intestinal permeability or small intestinal barrier function.

\section{Gastrointestinal complaints and exercise performance}

No gastrointestinal complaints were reported by the individuals when specifically asked for any GI disturbances experienced on the test days or the day following each test day. No differences in exercise performance, exhaustion or heart rate were observed between the two supplementation strategies (data not shown).

\section{DISCUSSION}

In the present randomized controlled study, the effect of oral citrulline supplementation prior to strenuous exercise on the development of splanchnic (micro)circulation, small intestinal injury and gut barrier function was studied. Oral L-citrulline supplementation prior to exercise was demonstrated to significantly increase plasma levels of citrulline and arginine in healthy athletes compared to placebo. As a result, elevated plasma arginine levels following oral L-citrulline supplementation increased the AAI, reflecting an increased availability of the NO-donor L-arginine, which persisted at least until one hour post-exercise. The increased arginine availability after oral L-citrulline supplementation was associated with preserved splanchnic perfusion during exercise compared to placebo, and reduced enterocyte damage. The presumed mechanism by which oral L-citrulline supplementation results in improved integrity in the splanchnic area is via increased intracellular availability of the NO-donor arginine. Intracellular arginine regeneration from citrulline via the conversion of citrulline by the ASS and ASL enzyme was previously found to be essential for eNOS-mediated NO production in endothelial cells (22), which may result in enhanced local blood flow through NO-mediated local vasodilatation (11).

Previously, we demonstrated that one hour of cycling at $70 \% \mathrm{~W}_{\max }$ rapidly increased $\mathrm{p}_{\mathrm{g}-\mathrm{a}} \mathrm{pCO}_{2}$ gap levels by more than $1 \mathrm{kPa}$, reflecting the development of splanchnic hypoperfusion during exercise in the fasted state (54). Although the increase in $\mathrm{p}_{\mathrm{g}-\mathrm{a}} \mathrm{pCO}_{2}$ gap was smaller in the present study than previously reported (54), it was comparable to other studies showing exercise-induced increases in $\mathrm{p}_{\mathrm{g}-\mathrm{a}} \mathrm{pCO}_{2}$ gap in healthy volunteers (38). Importantly, the present study reveals that a single pre-exercise dose of L-citrulline is able to avert the distinct increase in $\mathrm{p}_{\mathrm{g}-\mathrm{a}} \mathrm{pCO}_{2}$ gap 
level during exercise, which is observed after placebo supplementation. Although we could not measure local arginine availability in the healthy participants of the current study, experimental data indicated that intravenous L-citrulline supplementation is able to increase both plasma and intestinal arginine availability and increase the intracellular NO production in the intestine (59). In line with these studies, clinical studies indicated that oral supplementation of L-citrulline was able to increase plasma arginine concentrations and tissue NO-production without causing side effects $(17,18)$. Previously, we demonstrated that the small intestinal microcirculation was maintained during endotoxin-induced inflammation in mice if L-citrulline was applied intravenously (59). In line with these previous findings, the current study revealed that oral supplementation of L-citrulline prior to a trigger for splanchnic hypoperfusion, such as strenuous exercise, improves sublingual microcirculation, strongly suggesting improved intestinal microcirculation $(42,58)$.

Splanchnic hypoperfusion during strenuous cycling was reported to correlate with small intestinal injury in healthy athletes (54). Hypoperfusion-induced intestinal compromise occurring during strenuous exercise may hamper the athlete $(48,49)$ and can jeopardize early post-exercise recovery (53). We hypothesized that oral supplementation of L-citrulline improved splanchnic circulation during exercise, thereby reducing exercise-induced enterocyte injury. The present study revealed significantly lower plasma I-FABP levels during exercise in case of pre-exercise supplementation of L-citrulline compared to placebo, indicating that L-citrulline helps to reduce small intestinal cellular injury. In line with these data, L-citrulline was recently shown to reduce intestinal injury after experimentally induced small bowel obstruction, as reflected by reduced immunoglobulin concentrations in intestinal fluids of mice (2). High plasma I-FABP levels were found in patients surviving cardiac arrest, indicating the presence of profound small intestinal injury, most likely due to a period of little or no splanchnic perfusion (23). Interestingly, these high plasma I-FABP levels were associated with low circulating citrulline concentrations (23) which are considered a parameter of functional enterocyte mass (10), but which may also jeopardize arginine availability in this condition. These observations give emphasis to the promising results of oral L-citrulline supplementation to improve splanchnic perfusion and to diminish intestinal injury in situations of splanchnic hypoperfusion. Additionally, it insinuates that it might be useful to study the applicability of oral L-citrulline supplementation in other situations of splanchnic hypoperfusion, such as systemic hypotension, sepsis and maybe even high-risk surgery, which is associated with a high rate of complications $(3,16)$.

In contrast to the promising effects of oral L-citrulline supplementation on intestinal injury, no statistically significant effect could be found in the current study between L-citrulline and placebo supplementation with respect to GI permeability, reflecting intestinal barrier function. In our previous study, we found a small increase in small intestinal permeability after exercise (54). We consider that the small intestinal injury observed in the current study was not to such an extent that it led to loss of GI barrier function. Prolonged or more extensive reductions in splanchnic perfusion may lead to more profound GI compromise with loss of barrier integrity. 
The results of this study may be useful for athletes suffering from abdominal angina during strenuous exercise. It would be very interesting to test the effects of oral L-citrulline supplementation in symptomatic athletes. If L-citrulline supplementation is found to be beneficial in these athletes, it may also be interesting to extrapolate the results to asymptomatic athletes, and to find out whether oral L-citrulline supplementation also has advantages such as improved nutrient uptake and stimulation of early recovery. It was previously reported that oral L-citrulline supplementation improves muscle protein synthesis, comparable to the stimulating effect of leucine $(13,37)$. Both amino acids seem to exert this effect via the mTOR signaling pathway $(19,30)$. Interestingly, orally administered L-citrulline in combination with a low-protein diet in healthy volunteers was found to increase muscle protein synthesis compared to an isonitrogenous diet (13). Oral supplementation of L-citrulline may be used to improve nitrogen availability for via $\mathrm{L}$-arginine resynthesis from L-citrulline in the kidney (13). A high-protein intake is not likely to cause the same effect, because (a) citrulline is not present in a normal protein-rich diet, and (b) blood with a relatively high concentration of L-arginine floats from the intestines through the portal vein, the highly concentrated L-arginine leads to ureagenesis in the liver, which decreases the systemic L-arginine available for NO production (13). The results of oral L-citrulline supplementation during a high-protein diet may be more promising compared to L-arginine, as circulating L-arginine is scavenged by the liver-derived enzymes, whereas the liver is unable to scavenge citrulline, resulting in more arginine availability for NO-production (12).

In conclusion, the current study demonstrates that oral administration of L-citrulline prior to exercise preserves splanchnic perfusion and reduces intestinal injury during exercise. The mechanism probably entails increased arginine availability for NO-mediated vasodilatation. These data suggest oral L-citrulline supplementation to be a promising strategy to improve splanchnic perfusion and prevent intestinal injury in athletes. 


\section{REFERENCES}

1. Bahri S, Zerrouk N, Aussel C, Moinard C, Crenn P, Curis E, Chaumeil JC, Cynober L, and Sfar S. Citrulline: From metabolism to therapeutic use. Nutrition 2012.

2. Batista MA, Nicoli JR, Martins Fdos S, Machado JA, Arantes RM, Quirino IE, Correia MI, and Cardoso VN. Pretreatment with citrulline improves gut barrier after intestinal obstruction in mice. FPEN $\mathcal{F}$ Parenter Enteral Nutr 36: 69-76, 2012.

3. Beal AL, and Cerra FB. Multiple organ failure syndrome in the 1990s. Systemic inflammatory response and organ dysfunction. JAMA 271: 226-233, 1994.

4. Bjarnason I, MacPherson A, and Hollander D. Intestinal permeability: an overview. Gastroenterology 108: 15661581, 1995.

5. Bode-Boger SM, Boger RH, Galland A, Tsikas D, and Frolich JC. L-arginine-induced vasodilation in healthy humans: pharmacokinetic-pharmacodynamic relationship. Br f Clin Pharmacol 46: 489-497, 1998.

6. Boerma EC, Mathura KR, van der Voort PH, Spronk PE, and Ince C. Quantifying bedside-derived imaging of microcirculatory abnormalities in septic patients: a prospective validation study. Critical care (London, England) 9: R601-606, 2005.

7. Bradley SE, Childs AW, Combes B, Cournand A, Wade OL, and Wheeler HO. The effect of exercise on the splanchnic blood flow and splanchnic blood volume in normal man. Clin Sci (Lond) 15: 457-463, 1956.

8. Castillo L, Chapman TE, Yu YM, Ajami A, Burke JF, and Young VR. Dietary arginine uptake by the splanchnic region in adult humans. Am f Physiol 265: E532-539, 1993.

9. Coburn LA, Gong X, Singh K, Asim M, Scull BP, Allaman MM, Williams CS, Rosen MJ, Washington MK, Barry DP, Piazuelo MB, Casero RA, Jr., Chaturvedi R, Zhao Z, and Wilson KT. L-arginine supplementation improves responses to injury and inflammation in dextran sulfate sodium colitis. PLoS ONE 7: e33546, 2012.

10. Crenn P, Vahedi K, Lavergne-Slove A, Cynober L, Matuchansky C, and Messing B. Plasma citrulline: A marker of enterocyte mass in villous atrophy-associated small bowel disease. Gastroenterology 124: 1210-1219, 2003.

11. Curis E, Crenn P, and Cynober L. Citrulline and the gut. Current opinion in clinical nutrition and metabolic care 10: 620-626, 2007.

12. Curis E, Nicolis I, Moinard C, Osowska S, Zerrouk N, Benazeth S, and Cynober L. Almost all about citrulline in mammals. Amino Acids 29: 177-205, 2005.

13. Cynober L, Moinard C, and De Bandt JP. The 2009 ESPEN Sir David Cuthbertson. Citrulline: a new major signaling molecule or just another player in the pharmaconutrition game? Clinical nutrition (Edinburgh, Scotland) 29: 545-551, 2010.

14. De Backer D, Hollenberg S, Boerma C, Goedhart P, Buchele G, Ospina-Tascon G, Dobbe I, and Ince C. How to evaluate the microcirculation: report of a round table conference. Crit Care 11: R101, 2007.

15. de Oliveira EP, and Burini RC. Food-dependent, exercise-induced gastrointestinal distress. F Int Soc Sports Nutr 8: 12, 2011

16. Derikx JP, van Waardenburg DA, Thuijls G, Willigers HM, Koenraads M, van Bijnen AA, Heineman E, Poeze M, Ambergen T, van Ooij A, van Rhijn LW, and Buurman WA. New Insight in Loss of Gut Barrier during Major Non-Abdominal Surgery. PloS ONE 3: e3954, 2008.

17. El-Hattab AW, Emrick LT, Craigen WJ, and Scaglia F. Citrulline and arginine utility in treating nitric oxide deficiency in mitochondrial disorders. Mol Genet Metab 107: 247-252, 2012.

18. El-Hattab AW, Hsu JW, Emrick LT, Wong LJ, Craigen WJ, Jahoor F, and Scaglia F. Restoration of impaired nitric oxide production in MELAS syndrome with citrulline and arginine supplementation. Mol Genet Metab 105: 607-614, 2012.

19. Faure C, Raynaud-Simon A, Ferry A, Dauge V, Cynober L, Aussel C, and Moinard C. Leucine and citrulline modulate muscle function in malnourished aged rats. Amino Acids 42: 1425-1433, 2012.

20. Gazoni LM, Tribble CG, Zhao MQ, Unger EB, Farrar RA, Ellman PI, Fernandez LG, Laubach VE, and Kron IL. Pulmonary macrophage inhibition and inhaled nitric oxide attenuate lung ischemia-reperfusion injury. Ann Thorac Surg 84: 247-253, 2007.

21. Germann P, Braschi A, Della Rocca G, Dinh-Xuan AT, Falke K, Frostell C, Gustafsson LE, Herve P, Jolliet P, Kaisers U, Litvan H, Macrae DJ, Maggiorini M, Marczin N, Mueller B, Payen D, Ranucci M, Schranz D, Zimmermann R, and Ullrich R. Inhaled nitric oxide therapy in adults: European expert recommendations. Intensive care medicine 31: 1029-1041, 2005.

22. Goodwin BL, Solomonson LP, and Eichler DC. Argininosuccinate synthase expression is required to maintain nitric oxide production and cell viability in aortic endothelial cells. J Biol Chem 279: 18353-18360, 2004.

23. Grimaldi D, Guivarch E, Neveux N, Fichet J, Pene F, Marx JS, Chiche JD, Cynober L, Mira JP, and Cariou A. Markers of intestinal injury are associated with endotoxemia in successfully resuscitated patients. Resuscitation 84: 60-65, 2013. 
24. Grimble GK. Adverse gastrointestinal effects of arginine and related amino acids. $\mathcal{F}$ Nutr 137: 1693S-1701S, 2007.

25. Groner W, Winkelman JW, Harris AG, Ince C, Bouma GJ, Messmer K, and Nadeau RG. Orthogonal polarization spectral imaging: a new method for study of the microcirculation. Nat Med 5: 1209-1212, 1999.

26. Ince C. The microcirculation unveiled. American journal of respiratory and critical care medicine 166: 1-2, 2002.

27. Knowles TP, Mullin RA, Hunter JA, and Douce FH. Effects of syringe material, sample storage time, and temperature on blood gases and oxygen saturation in arterialized human blood samples. Respiratory care 51: 732736, 2006.

28. Kolkman JJ, Groeneveld AB, and Meuwissen SG. Effect of ranitidine on basal and bicarbonate enhanced intragastric PCO2: a tonometric study. Gut 35: 737-741, 1994.

29. Kuipers H, Verstappen FT, Keizer HA, Geurten P, and van Kranenburg G. Variability of aerobic performance in the laboratory and its physiologic correlates. Int F Sports Med 6: 197-201, 1985.

30. Le Plenier S, Walrand S, Noirt R, Cynober L, and Moinard C. Effects of leucine and citrulline versus nonessential amino acids on muscle protein synthesis in fasted rat: a common activation pathway? Amino Acids 43: 1171-1178, 2012.

31. Lieberman JM, Sacchettini J, Marks C, and Marks WH. Human intestinal fatty acid binding protein: report of an assay with studies in normal volunteers and intestinal ischemia. Surgery 121: 335-342, 1997.

32. Luiking YC, Engelen MP, and Deutz NE. Regulation of nitric oxide production in health and disease. Current opinion in clinical nutrition and metabolic care 13: 97-104, 2010.

33. Martin MJ, Jimenez MD, and Motilva V. New issues about nitric oxide and its effects on the gastrointestinal tract. Curr Pharm Des 7: 881-908, 2001.

34. Moinard C, Nicolis I, Neveux N, Darquy S, Benazeth S, and Cynober L. Dose-ranging effects of citrulline administration on plasma amino acids and hormonal patterns in healthy subjects: the Citrudose pharmacokinetic study. Br F Nutr 99: 855-862, 2008.

35. Morris SM, Jr. Enzymes of arginine metabolism. J Nutr 134: 2743S-2747S; discussion 2765S-2767S, 2004.

36. Moses FM. Exercise-associated intestinal ischemia. Curr Sports Med Rep 4: 91-95, 2005.

37. Osowska S, Duchemann T, Walrand S, Paillard A, Boirie Y, Cynober L, and Moinard C. Citrulline modulates muscle protein metabolism in old malnourished rats. Am F Physiol Endocrinol Metab 291: E582-586, 2006.

38. Otte JA, Oostveen E, Geelkerken RH, Groeneveld AB, and Kolkman JJ. Exercise induces gastric ischemia in healthy volunteers: a tonometry study. F Appl Physiol 91: 866-871, 2001.

39. Pelsers MM, Hermens WT, and Glatz JF. Fatty acid-binding proteins as plasma markers of tissue injury. Clinica chimica acta; international journal of clinical chemistry 352: 15-35, 2005.

40. Peters HP, Bos M, Seebregts L, Akkermans LM, van Berge Henegouwen GP, Bol E, Mosterd WL, and de Vries WR. Gastrointestinal symptoms in long-distance runners, cyclists, and triathletes: prevalence, medication, and etiology. The American journal of gastroenterology 94: 1570-1581, 1999.

41. Poeze M, Bruins MJ, Kessels F, Luiking YC, Lamers WH, and Deutz NE. Effects of L-arginine pretreatment on nitric oxide metabolism and hepatosplanchnic perfusion during porcine endotoxemia. Am f Clin Nutr 93: 1237-1247, 2011.

42. Pranskunas A, Koopmans M, Koetsier PM, Pilvinis V, and Boerma EC. Microcirculatory blood flow as a tool to select ICU patients eligible for fluid therapy. Intensive care medicine 2012.

43. Puiman PJ, Stoll B, van Goudoever JB, and Burrin DG. Enteral arginine does not increase superior mesenteric arterial blood flow but induces mucosal growth in neonatal pigs. $\mathcal{F}$ Nutr 141: 63-70, 2011.

44. Qamar MI, and Read AE. Effects of exercise on mesenteric blood flow in man. Gut 28: 583-587, 1987.

45. Romero MJ, Platt DH, Caldwell RB, and Caldwell RW. Therapeutic use of citrulline in cardiovascular disease. Cardiovascular drug reviews 24: 275-290, 2006.

46. Smith HA, Canter JA, Christian KG, Drinkwater DC, Scholl FG, Christman BW, Rice GD, Barr FE, and Summar ML. Nitric oxide precursors and congenital heart surgery: a randomized controlled trial of oral citrulline. F Thorac Cardiovasc Surg 132: 58-65, 2006.

47. Spronk PE, Ince C, Gardien MJ, Mathura KR, Oudemans-van Straaten HM, and Zandstra DF. Nitroglycerin in septic shock after intravascular volume resuscitation. Lancet 360: 1395-1396, 2002.

48. Ter Steege RW, Geelkerken RH, Huisman AB, and Kolkman JJ. Abdominal symptoms during physical exercise and the role of gastrointestinal ischaemia: a study in 12 symptomatic athletes. Br $\mathcal{F}$ Sports Med 2011.

49. ter Steege RW, Van der Palen J, and Kolkman JJ. Prevalence of gastrointestinal complaints in runners competing in a long-distance run: an internet-based observational study in 1281 subjects. Scand F Gastroenterol 43: 14771482, 2008.

50. van de Poll MC, Ligthart-Melis GC, Boelens PG, Deutz NE, van Leeuwen PA, and Dejong CH. Intestinal and hepatic metabolism of glutamine and citrulline in humans. The fournal of physiology 581: 819-827, 2007. 
51. van de Poll MC, Siroen MP, van Leeuwen PA, Soeters PB, Melis GC, Boelens PG, Deutz NE, and Dejong CH. Interorgan amino acid exchange in humans: consequences for arginine and citrulline metabolism. Am $\mathcal{F}$ Clin Nutr 85: 167-172, 2007.

52. van Eijk HM, Rooyakkers DR, and Deutz NE. Rapid routine determination of amino acids in plasma by highperformance liquid chromatography with a 2-3 microns Spherisorb ODS II column. $f$ Chromatogr 620: 143$148,1993$.

53. van Wijck K, Lenaerts K, Grootjans J, Wijnands KA, Poeze M, van Loon LJ, Dejong CH, and Buurman WA. Physiology and pathophysiology of splanchnic hypoperfusion and intestinal injury during exercise: strategies for evaluation and prevention. American journal of physiology Gastrointestinal and liver physiology 303: G155-168, 2012.

54. van Wijck K, Lenaerts K, van Loon LJ, Peters WH, Buurman WA, and Dejong CH. Exercise-induced splanchnic hypoperfusion results in gut dysfunction in healthy men. PLoS ONE 6: e22366, 2011.

55. van Wijck K, van Eijk HM, Buurman WA, Dejong CH, and Lenaerts K. Novel analytical approach to a multisugar whole gut permeability assay. F Chromatogr B Analyt Technol Biomed Life Sci 879: 2794-2801, 2011.

56. van Wijck K, Verlinden TJ, van Eijk HM, Dekker J, Buurman WA, Dejong CH, and Lenaerts K. Novel multisugar assay for site-specific gastrointestinal permeability analysis: A randomized controlled crossover trial. Clinical nutrition 2012.

57. Verdant CL, De Backer D, Bruhn A, Clausi CM, Su F, Wang Z, Rodriguez H, Pries AR, and Vincent JL. Evaluation of sublingual and gut mucosal microcirculation in sepsis: a quantitative analysis. Crit Care Med 37: 2875$2881,2009$.

58. Verdant CL, De Backer D, Bruhn A, Clausi CM, Su F, Wang Z, Rodriguez H, Pries AR, and Vincent JL. Evaluation of sublingual and gut mucosal microcirculation in sepsis: a quantitative analysis. Critical care medicine 37 : 2875-2881, 2009.

59. Wijnands KA, Vink H, Briede JJ, van Faassen EE, Lamers WH, Buurman WA, and Poeze M. Citrulline a more suitable substrate than arginine to restore NO production and the microcirculation during endotoxemia. PloS ONE 7: e37439, 2012.

60. Windmueller HG, and Spaeth AE. Source and fate of circulating citrulline. Am J Physiol 241: E473-480, 1981.

61. Wu G, and Meininger CJ. Regulation of L-arginine synthesis from L-citrulline by L-glutamine in endothelial cells. Am f Physiol 265: H1965-1971, 1993. 


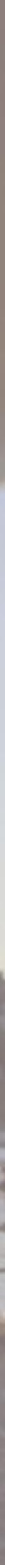

ChaptER 11

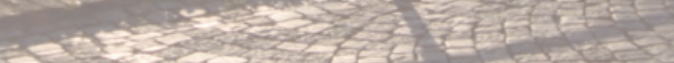

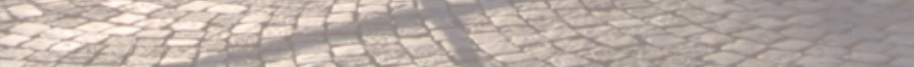

C.

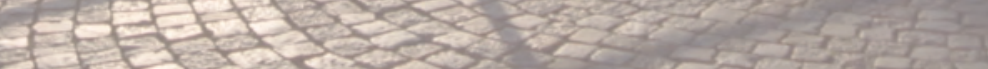

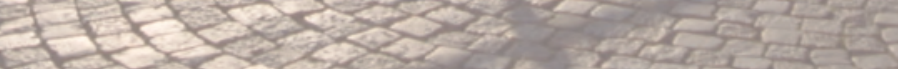

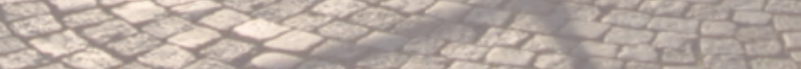

$32025 \mathrm{seng}$ 
The integrity of the gastrointestinal (GI) tract is tightly controlled by its complex and highly dynamic architecture $(22,33)$, resulting in a remarkable flexibility and regenerative capacity to adapt to for example an increased metabolic demand (8) and to recuperate if damage has occurred (12). An important factor that can compromise GI integrity is splanchnic hypoperfusion (46). The experimental in vivo studies described in this thesis focused on splanchnic hypoperfusion and GI integrity loss in man. In the current chapter, I will elaborate on the physiological and pathophysiological aspects of splanchnic hypoperfusion and ischemia, and discuss GI integrity in this context.

\section{Splanchnic hypoperfusion and ischemia}

Splanchnic hypoperfusion is a state in which blood flow in the splanchnic area is reduced to a suboptimal level. Hypoperfusion is inextricably linked to ischemia, a state in which the splanchnic circulation is restricted to such an extent that mucosal hypoxia and depletion of adenosine triphosphate (ATP) occur, leading to local acidosis and tissue injury (12). Both entities are the result of a mismatch of blood supply and demand in the splanchnic area, which may be induced by either a critically reduced blood supply, an increased metabolic demand, or both. Many conditions exist in which the amount of blood flowing through the splanchnic area does not meet the requirements for nutrients and oxygen, ranging from mild to severe hypoperfusion. Included in the current thesis are physiological and pathophysiological conditions of critically reduced splanchnic perfusion, i.e. during exercise-induced redistribution of blood flow at the expense of the splanchnic area in athletes (chapter 7), during exercise with prior intake of non-steroidal anti-inflammatory drugs (NSAIDs; chapter 9) and during acute obstruction of splanchnic vessel(s) in patients with intestinal ischemia (chapter 3). I will discuss the interplay between these physiological and pathophysiological manifestations of splanchnic hypoperfusion and GI integrity loss.

\section{Exercise-induced splanchnic hypoperfusion and GI integrity: physiology or pathophysiology?}

The splanchnic vascular system has a remarkable capacity to adapt to physiological stressors via regulation of the mesenteric vascular resistance by neuroendocrine, humoral, and paracrine mechanisms (34). During strenuous exercise, splanchnic vasoconstriction occurs as a result of a norepinephrine-induced increase in splanchnic vascular resistance $(17,35,64)$, thereby rapidly redistributing blood from the splanchnic area towards heart, lungs, active muscle and skin (5, 43) to enable athletic performance and evaporate excessive heat via the skin. Doppler ultrasound and contrast extraction studies revealed a critical reduction in hepatosplanchnic blood flow during exercise by as much as $80 \%$ during heavy physical exercise (5). Animal studies have demonstrated that ischemia occurs when splanchnic blood flow decreases to less than $40-50 \%$ of normal flow $(25,29)$. In line, it was reported that heavy physical exercise resulted in splanchnic hypoperfusion and ischemia in healthy $(39,43)$. 
These data give rise an intriguing question: Is exercise-induced splanchnic hypoperfusion a physiological or pathophysiological phenomenon?

In the study described in chapter 7 , the pattern of the exercise-induced hypoperfusion during prolonged exercise was studied. Hypoperfusion occurred within the first ten minutes of strenuous exercise, was maintained during the full duration of the 60-minute exercise bout, and was rapidly reversed in the first ten minutes post-exercise (chapter 7), emphasizing the highly responsive adaptive capacity of the splanchnic vascular bed. Alternatively, previous studies reported increased postprandial mesenteric blood flow by as much as $60-250 \%$ to meet the increased circulatory demand associated with digestion and absorption (35), lasting for approximately 2-3h before returning to baseline levels (34). The gut is built to adapt to such changes in splanchnic perfusion, and the quick restoration of blood flow to baseline levels, after fluctuations due to food intake or exercise, strongly suggests that we should consider exercise-induced splanchnic hypoperfusion to be a physiological phenomenon.

Exercise-induced splanchnic hypoperfusion is closely related to loss of enterocyte integrity in the small intestine in our human exercise study (chapter 7), indicating that the physiologically induced hypoperfusion led to GI integrity loss. Critical reductions of the splanchnic blood flow have been demonstrated to enhance the physiological low-flow state in the upper half of the small intestinal villi, resulting in disruption of mucosal integrity $(3,4)$. The mature enterocytes located at the upper half of the intestinal villi are the first cells to be affected, while the undifferentiated intestinal epithelial cells are relatively safe in the crypt and lower part of the villus. In the human exercise studies described in this thesis, we observed significant increases in circulating intestinal fatty acid binding protein (I-FABP) levels (chapter 7-10), reflecting loss of cellular integrity of the especially the mature enterocytes. These differentiated, mature enterocytes are cells that have gained pro-apoptic properties during their journey along the crypt-villus axis (16), making them prone to undergo apoptosis as part of the physiological process of constant epithelial renewal and anoiksis. Deprivation of oxygen in the villus tip as a result of hypoperfusion is one of the triggers to initiate this physiological process (3), suggesting that hypoperfusioninduced loss of cellular integrity, triggered by exercise, may be a physiological phenomenon. Of the athletes participating in our exercise studies, $87 \%$ (26/30) had small intestinal cellular injury after exercise, reflected by a considerable increase in plasma (I-FABP), defined as a rise of at least 35\% above pre-exercise levels.

Compromised intestinal integrity caused by significant reductions in splanchnic flow may comprise more than cell damage. Previous studies in animals reported that energy depletion following ischemia/reperfusion injury led to relocalization of the apical cell membrane and disruption of the apical actin cytoskeleton $(1,51)$, leading to loss of intestinal integrity with paracellular leakage of microbial products and other potentially harmful luminal components (15). More recently, intestinal cytoskeleton disruption and tight junction integrity loss were reported in rats following intestinal hypoperfusion (54). Furthermore, gut barrier dysfunction with compromised 
enterocyte integrity and tight junction protein loss was observed following intestinal mucosal hypoperfusion in patients undergoing major non-abdominal surgery (13). Based on these studies, it was hypothesized that exercise-induced intestinal hypoperfusion and the subsequent small intestinal injury compromise gut barrier function. Urinary tight junction protein levels remained undetectable in the majority of athletes after $1 \mathrm{~h}$ of cycling at $70 \%$ of maximum capacity (unpublished data), and no significant changes in plasma endotoxin antibodies were observed (supplemental data chapter 7), suggesting that the extent of injury was not enough to induce profound tight junction integrity loss with bacterial translocation. These findings corroborate outcomes of permeability assessment using our sensitive multi-sugar assay in the same healthy athletes, showing that plasma appearance of sugar probes and plasma permeability ratios increased, while urinary ratios of these probes were marginally elevated compared to rest (chapter 7 ). The small changes in GI permeability after exercise in healthy athletes can partly explain why data on this subject are controversial $(27,28,32,59,60)$. Only the use of a sensitive permeability test may detect the small changes that occur during strenuous exercise. The variety in exercise-related permeability data may therefore also partly be explained by the variety of tests and experimental study designs used to assess alterations in intestinal permeability after exercise, including the moment of sugar probe intake. The modest permeability changes observed after one hour of cycling (chapter 7) are apparently part of the physiological fluctuations in GI permeability. More profound increases in GI permeability were observed if cycling was combined with prior intake of NSAIDs (chapter 9), indicating aggravated loss of gut barrier function in healthy athletes. Does the combination of exercise and NSAIDs raise the exercise-induced GI compromise to a pathophysiological level? The fact that I-FABP levels approximate baseline levels one hour postexercise points in the opposite direction. Our exercise studies emphasize the capacity of the human gut to withstand short periods of splanchnic hypoperfusion and other potentially harmful influences such as NSAID consumption without major GI compromise. Nonetheless, it is important to point athletes and trainers to the potentially harmful NSAIDs, and to discourage the use of these drugs as ergogenic aids, especially in the absence of a clear medical indication.

Recurrent episodes of intestinal injury and barrier integrity loss (caused by exercise or a combination of exercise and NSAIDs) require a remarkable regenerative capacity of the gut. While exercise itself increases the need for adequate energy supply and nutrient uptake in the intestine, such renewal of the epithelium also increases the metabolic demand of the GI system. Data of the study described in chapter 7 gave rise to the intriguing question whether the exerciseinduced intestinal injury also compromises the digestive and absorptive capacities of the GI tract. In the study described in chapter 8 , we used specifically produced intrinsically labeled dairy protein in combination with continuous intravenous tracer infusion to confirm that the exercise-induced intestinal injury impairs in vivo dietary protein digestion and absorption in the acute post-exercise recovery phase. Especially in professional athletes and in patients, such impaired uptake of energy and nutrients caused by hypoperfusion-induced small intestinal injury 
in combination with an increased metabolic demand may delay early and complete recovery. In line with the extent of intestinal injury and gut barrier integrity loss, the observed changes in the absorptive function of the gut are considered to be within the physiological range.

Naturally, there are cases in which strenuous exercise led to extensive mucosal injury, including mucosal erosions $(7,42)$, (bloody) diarrhea and haemorrhagic colitis (36-38). Additionally, healthy individuals with exercise-induced pain that is explained by the celiac artery compression syndrome (CACS), in which flow through the celiac artery is significantly reduced due to external compression most often by a crus of the diaphragm, are considered to suffer from a pathophysiological condition that is relatively easily resolved (14). In these cases, the physiological exercise-induced in vivo changes have evolved to pathophysiological phenomena.

\section{Splanchnic hypoperfusion and GI integrity in other conditions}

Splanchnic hypoperfusion induced by exercise in healthy individuals leads to small intestinal injury and loss of GI integrity within the physiological range (chapter 7 and 9). Other examples in which splanchnic hypoperfusion occurs are trauma patients especially when in shock (10), burn victims (30), and patients undergoing cardiopulmonary bypass (40, 41, 47). Additionally, patients undergoing major non-abdominal surgery were described to develop gut barrier dysfunction associated with systemic hypotension and splanchnic hypoperfusion if no specific attention was paid to maintain the mean arterial pressure during surgery above $60 \mathrm{~mm} \mathrm{Hg}$ (13). The hypoperfusion-associated small intestinal injury during surgery in these patients was of similar extent as the exercise-induced intestinal damage that was observed in healthy athletes of our exercise studies (chapter 7 and 10). It seems logical that patients with cardiopulmonary dysfunction have an increased risk to develop splanchnic hypoperfusion for example during surgery, even when they are relatively fit and found to be eligible for surgery according to the classifications of the American Society of Anesthesiologists (9). In case of chronic underlying disease such as chronic heart failure (CHF) or obstructive pulmonary disease, hypoperfusion likely results in more profound or prolonged intestinal compromise, due to a more delicate balance in splanchnic perfusion and GI integrity, and a reduced capacity to recover following recurrent episodes of hypoperfusion-induced intestinal injury (48, 49). CHF leads to increased sympathetic activity, which decreases splanchnic blood flow (49). In line with these results, intestinal ischemia has been observed in patients with CHF during lowintensity exercise (26), emphasizing the susceptibility of this population to GI compromise due to decreased splanchnic circulation.

Severely impaired splanchnic perfusion in critically ill patients in the intensive care unit is associated with poor outcome $(23,58)$. Specific routine nursing procedures such as airway suctioning, determining the patient's level of sedation, and changing the patient's position were associated with decreases in hepatosplanchnic oxygen saturation, suggesting impaired perfusion in this area (23). Other therapeutic interventions that may critically reduce splanchnic circulation are the administration of vasoactive drugs such as vasopressine (57) and hemodialysis (24). 
Patients with acute intestinal ischemia probably form the archetypical population at the end of the splanchnic hypoperfusion scale, i.e. severe splanchnic hypoperfusion. In the study described in chapter 3, we have demonstrated that these patients have very high levels of circulating gut-derived FABPs, indicating profound intestinal damage, which may be located in a specific part of the intestine or may affect the whole intestinal area. From the patients with intestinal ischemia in this study, 7 patients died due to too profound damage or too late intervention, indicating a serious life-threatening condition. Studies aiming at improving the diagnostic workup for splanchnic hypoperfusion and its sequelae in athletes during and after exercise may help to develop strategies to enable early diagnosis of the problem, to improve splanchnic blood flow, and reduce or prevent hypoperfusion-induced small intestinal injury and loss of barrier integrity in patients.

\section{Improving the diagnostic work-up of splanchnic hypoperfusion and its sequelae}

The anatomy of the splanchnic circulation, including any vascular abnormalities may be visualized using computed tomography angiography, classic or magnetic resonance angiography, or transcutaneous abdominal duplex ultrasound (35). Alternative emerging techniques to assess GI ischemia, analyze the mucosal oxygen saturation of hemoglobin in red blood cells of the intestinal microcirculation by emission and detection of backscattered light during endoscopy (chapter 2). The only functional test currently available to determine the presence of splanchnic hypoperfusion is gastric air tonometry (35). The latter is considered most useful in combination with assessment of the sequelae of splanchnic hypoperfusion through determination of GI integrity and functionality.

GI barrier integrity assessment was briefly discussed in the light of exercise-induced intestinal compromise. Barrier integrity can be assessed by of (a) urinary or plasma levels of components of the GI barrier, by (b) plasma concentrations or urinary excretion of permeability probes after oral ingestion, and (c) bacterial products in the systemic circulation (21). In chapter 3, the loss of intestinal cellular integrity in patients with intestinal ischemia was determined using intestinal fatty acid binding proteins, which are small, $15-\mathrm{kD}$ cytosolic proteins present in the intact enterocytes of the intestine. These proteins, especially I-FABP, have been found to be reliable parameters of small intestinal cellular injury in many different situations, including in patients with intestinal ischemia (chapter 3), celiac disease (63), intestinal necrosis after aortic surgery (61) and during exercise in healthy individuals (chapter 7-10). However, the GI barrier consists of many more components than the intestinal epithelial cells (IECs), one example being the highly dynamic tight junctional complexes that seal the paracellular space between the epithelial cells.

Intestinal ischemia and reperfusion have been found to change myosin light-chain kinase levels, suggesting contraction of the actomyosin skeleton in the IECs and tight junction opening at sites where IECs have lost contact with the basal membrane (20). In case of prolonged ischemia, epithelial barrier loss was observed, with loss of tight junction proteins and significant translo- 
cation of bacterial endotoxin (19). Accordingly, both determination of tight junction proteins such as claudin-3 in urine or plasma and determination of circulating endotoxin levels can be used to assess GI barrier function (55), but only in case of profound barrier integrity loss, i.e. in mainly in pathophysiological conditions. In addition, results should be interpreted with caution, as these components are not specific for the intestine.

Novel GI permeability tests were developed in an attempt to improve the available diagnostics for site-specific GI barrier integrity assessment both in physiological and pathophysiological conditions. Three tests for GI permeability assessment were described in this thesis, all building on the same principle: urinary or plasma permeability probe levels are determined following oral intake of these probes by the test subject (2). The application of differently sized probes allows assessment of the severity of the GI barrier dysfunction, while the use of degradable and nondegradable probes enables analysis of specific parts of the GI system (2). The three tests described in this thesis are a permeability test based on polyethylene glycols (PEG), a novel multi-sugar (MS) assay, and the classical dual sugar (DS) lactulose-rhamnose test. Since we optimized the method of detection and changed the composition of the PEG test and MS test, the first question to be answered was whether these novel tests could detect experimentally increased GI permeability in healthy individuals. Test outcomes and accuracy were evaluated and compared with the most commonly used DS test. The study results reported in chapter 4 demonstrated that the PEG test and DS test had similar accuracy rates when used for permeability analysis in healthy individuals after NSAID intake. Since we sought to improve the techniques available for permeability testing, we were not satisfied with these results. In chapter 5, our novel analytical approach towards a multi-sugar permeability assay is described, which is validated in chapter 6 . With a reduced lactulose load, additional sugars for site-specific GI permeability analysis, and the possibility to determine sugar probe levels in plasma samples to allow detection of smaller, transient permeability changes, this test has clear advantages over the other two permeability tests (chapter 5 and 6). The accuracy of this test in healthy individuals with experimentally increased GI permeability was slightly higher than the classical DS test. This, in combination with the previously mentioned advantages, led us to conclude that the MS test is a promising tool for accurate, site-specific GI permeability analysis. The MS test gave reliable results in healthy individuals under different circumstances, such as during strenuous exercise and after intake of NSAIDs. Future research is needed to prove whether the MS test provides advantages for permeability assessment in pathophysiological conditions as well. 


\section{Strategies to treat or prevent hypoperfusion-induced GI integrity loss}

Preventive and therapeutic strategies for splanchnic hypoperfusion-induced GI integrity loss are important in case of pathophysiological hypoperfusion. Additionally, such strategies may be beneficial in specific physiological conditions such as professional athletes and symptomatic recreationally active individuals. Up to $70 \%$ of athletes have exercise-associated GI symptoms (44), and studies so far have mainly focused on the prevention of these symptoms. To my knowledge, no studies have demonstrated a direct relationship between exercise-induced GI integrity loss and abdominal symptoms. However, most strategies to resolve the exercise-induced problems are likely to reduce intestinal injury as well, potentially due to their beneficial effect on splanchnic blood flow during exercise and directly post-exercise. Recurrent episodes of intestinal injury and barrier integrity loss require a remarkable regenerative capacity of the gut. While exercise itself increases the need for adequate energy supply and nutrient uptake in the intestine, such renewal of the epithelium also increases the metabolic demand of the GI system. In chapter 8 , we have demonstrated that exercise and the associated small intestinal injury may impair the digestion and absorption of proteins consumed immediately post-exercise in healthy young athletes compared to resting conditions. Especially in professional athletes and in patients, such impaired uptake of nutrients caused by hypoperfusion-induced small intestinal injury in combination with an increased metabolic demand may delay early and complete recovery. Training the gut for the intake of small amounts of food during exercise-induced splanchnic hypoperfusion is thought to maintain blood flow at a higher level than in fasted state (53) due to the food-induced physiological rise in GI blood flow (65). Additionally, specific nutritional strategies may be initiated to improve splanchnic perfusion, reduce small intestinal integrity loss, and maintain adequate energy intake in athletes and patients. An overview of such strategies is included in chapter 2 of this thesis. As stated, most of the supplementation strategies are still being developed and should be tested in larger groups of athletes and patients before clear recommendations for individual use can be done.

Oral supplementation of L-citrulline 30 minutes prior to cycling was observed to maintain adequate splanchnic perfusion during cycling, while oral supplementation of L-alanine prior to exercise did not prevent from exercise-induced splanchnic hypoperfusion (chapter 10). Preserved splanchnic blood flow after L-citrulline supplementation reduced the extent of exercise-induced loss of enterocyte integrity. Interestingly, five individuals of the citrulline supplementation study also participated in the study in which no supplementation was given prior to exercise (chapter 7), allowing a comparison of data with and without nutrient supplementation. It was clear that the increase in gap ${ }_{\mathrm{g}-\mathrm{a}} \mathrm{pCO}_{2}$ levels, reflecting splanchnic hypoperfusion, was more obvious during exercise if no supplementation was given prior to exercise compared to data after L-alanine or L-citrulline supplementation, respectively (Figure 1A). In line with these data, plasma I-FABP levels during exercise were higher in case of cycling without supplementation, while cycling with prior intake of either L-alanine of L-citrulline resulted in lower plasma I-FABP levels during 
A
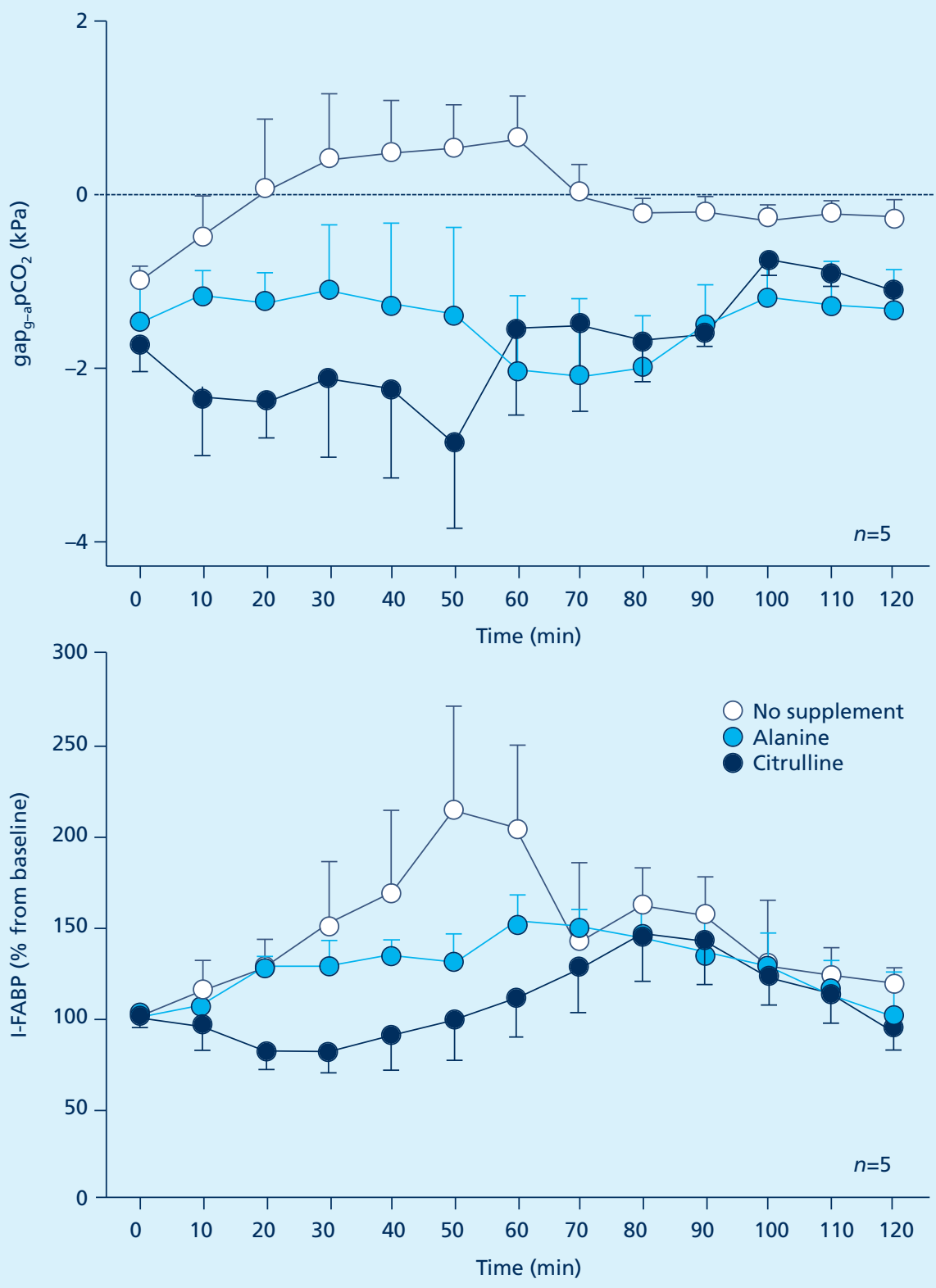

Figuur 1: Oral intake of amino acids, especially citrulline, improves splanchnic perfusion and preserves intestinal integrity during exercise

A) Pre-exercise oral intake of amino acids, especially citrulline, preserves gap ${ }_{\mathrm{g}-\mathrm{a}} \mathrm{pCO}_{2}$ levels at pre-exercise levels compared to gap $\mathrm{g}_{\mathrm{g}-\mathrm{a}} \mathrm{pCO}_{2}$ levels measured in the same individuals during exercise without prior oral supplementation. B) Plasma I-FABP levels are maintained at pre-exercise levels if prior to exercise, oral supplementation of L-alanine of L-citrulline is administered, while cycling without supplementation raised plasma I-FABP levels. Circles represent mean data of five participants, with the SEM as black lines. 
exercise, suggesting better preserved small intestinal integrity (Figure 1B). These data suggest that oral intake of amino acids, and possibly other nutrients, prior to exercise improves splanchnic perfusion and GI integrity during exercise compared to the situation in which no supplementation is used. Maintenance of splanchnic blood flow may also stimulate nutrient uptake during exercise and in the acute post-exercise recovery phase, but specific future studies are warranted to prove such an effect after pre-exercise oral citrulline supplementation. Additionally, while we observed a $100 \%$ tolerance for the oral intake of citrulline and the placebo alanine prior to exercise, future studies should take into account the additional burden that a pre-exercise oral citrulline upload may put on the gastrointestinal tract.

An additional potentially useful nutritional supplementation strategy is lipid-rich enteral nutrition, which has been shown to prevent GI integrity loss and subsequent intestinal inflammation during an episode of splanchnic hypoperfusion in rats (11). Recently, these data have been confirmed in a highly interesting study using lipid-rich enteral nutrition in experimentally-induced endotoxemia in otherwise healthy individuals (31). These effects have yet to be confirmed in athletes. Studies on lipid-rich nutrition in athletes have mainly focused on the utilization of lipids for enhancement of fat oxidation during exercise in order to improve endurance capacity (6, 18), but an additional beneficial effect on endurance capacity via stimulation of splanchnic blood flow, maintained GI integrity and preserved digestive and absorptive GI functioning is well possible. It would be interesting to apply the recent experiences on lipid-rich enteral nutrition to other situations with splanchnic hypoperfusion, more specifically in case of exercise-induced splanchnic hypoperfusion.

In addition to food intervention strategies, both in athletes and in (surgical) patients, adequate hydration helps to maintain circulating blood volume $(45,50,52,62)$, thereby contributing to the prevention of GI compromise. Avoiding the use of NSAIDs also helps to avoid profound intestinal damage in case of splanchnic hypoperfusion in athletes (chapter 9) and may do so in patients with hypoperfusion. An effective measure to improve splanchnic perfusion in surgical patients was demonstrated by Thuijls et al (56). Maintenance of the mean arterial pressure above the critical value of $60 \mathrm{~mm} \mathrm{Hg}$ was reported to secure splanchnic perfusion throughout the surgical procedure, thereby preserving mucosal integrity of the small intestine (56). This strategy may also be used in critically ill patients and in trauma patients on the intensive care unit or during resuscitation.

In conclusion, at this stage various promising strategies are available that may reduce or even prevent GI compromise associated with splanchnic hypoperfusion. Future studies are warranted to determine the specific usefulness of these strategies for application in athletes and patients. 


\section{REFERENCES}

1. Ashworth SL, Sandoval RM, Hosford M, Bamburg JR, and Molitoris BA. Ischemic injury induces ADF relocalization to the apical domain of rat proximal tubule cells. American journal of physiology Renal physiology 280: F886-894, 2001.

2. Bjarnason I, MacPherson A, and Hollander D. Intestinal permeability: an overview. Gastroenterology 108: 15661581, 1995.

3. Blikslager AT. Life in the gut without oxygen: adaptive mechanisms and inflammatory bowel disease. Gastroenterology 134: 346-348, 2008.

4. Blikslager AT, Moeser AJ, Gookin JL, Jones SL, and Odle J. Restoration of barrier function in injured intestinal mucosa. Physiological reviews 87: 545-564, 2007.

5. Bradley SE, Childs AW, Combes B, Cournand A, Wade OL, and Wheeler HO. The effect of exercise on the splanchnic blood flow and splanchnic blood volume in normal man. Clin Sci (Lond) 15: 457-463, 1956.

6. Brouns F, and van der Vusse GJ. Utilization of lipids during exercise in human subjects: metabolic and dietary constraints. The British journal of nutrition 79: 117-128, 1998.

7. Choi SC, Choi SJ, Kim JA, Kim TH, NahYH, Yazaki E, and Evans DF. The role of gastrointestinal endoscopy in long-distance runners with gastrointestinal symptoms. European journal of gastroenterology $\&$ hepatology 13: 10891094, 2001.

8. Cox GR, Clark SA, Cox AJ, Halson SL, Hargreaves M, Hawley JA, Jeacocke N, Snow RJ, Yeo WK, and Burke LM. Daily training with high carbohydrate availability increases exogenous carbohydrate oxidation during endurance cycling. Fournal of applied physiology 109: 126-134, 2010.

9. Davenport DL, Bowe EA, Henderson WG, Khuri SF, and Mentzer RM, Jr. National Surgical Quality Improvement Program (NSQIP) risk factors can be used to validate American Society of Anesthesiologists Physical Status Classification (ASA PS) levels. Annals of surgery 243: 636-641; discussion 641-634, 2006.

10. de Haan JJ, Lubbers T, Derikx JP, Relja B, Henrich D, Greve JW, Marzi I, and Buurman WA. Rapid development of intestinal cell damage following severe trauma: a prospective observational cohort study. Critical care 13: R86, 2009.

11. de Haan JJ, Thuijls G, Lubbers T, Hadfoune M, Reisinger K, Heineman E, Greve JW, and Buurman WA. Protection against early intestinal compromise by lipid-rich enteral nutrition through cholecystokinin receptors. Critical care medicine 38: 1592-1597, 2010.

12. Derikx JP, Matthijsen RA, de Bruine AP, van Bijnen AA, Heineman E, van Dam RM, Dejong CH, and Buurman WA. Rapid reversal of human intestinal ischemia-reperfusion induced damage by shedding of injured enterocytes and reepithelialisation. PloS ONE 3: e3428, 2008.

13. Derikx JP, van Waardenburg DA, Thuijls G, Willigers HM, Koenraads M, van Bijnen AA, Heineman E, Poeze M, Ambergen T, van Ooij A, van Rhijn LW, and Buurman WA. New Insight in Loss of Gut Barrier during Major Non-Abdominal Surgery. PloS ONE 3: e3954, 2008.

14. Duffy AJ, Panait L, Eisenberg D, Bell RL, Roberts KE, and Sumpio B. Management of median arcuate ligament syndrome: a new paradigm. Annals of vascular surgery 23: 778-784, 2009.

15. Fink MP DR. Epithelial barrier dysfunction: a unifying theme to explain the pathogenesis of multiple organ dysfunction at the cellular level. Critical Care Clinic 21: 177-196, 2005.

16. Gassler N, Roth W, Funke B, Schneider A, Herzog F, Tischendorf JJ, Grund K, Penzel R, Bravo IG, Mariadason J, Ehemann V, Sykora J, Haas TL, Walczak H, Ganten T, Zentgraf H, Erb P, Alonso A, Autschbach F, Schirmacher P, Knuchel R, and Kopitz J. Regulation of enterocyte apoptosis by acyl-CoA synthetase 5 splicing. Gastroenterology 133: 587-598, 2007.

17. Gil SM, Yazaki E, and Evans DF. Aetiology of running-related gastrointestinal dysfunction. How far is the finishing line? Sports medicine (Auckland, NZ) 26: 365-378, 1998.

18. Gonzalez JT, and Stevenson EJ. New perspectives on nutritional interventions to augment lipid utilisation during exercise. The British journal of nutrition 107: 339-349, 2012.

19. Grootjans J, Lenaerts K, Derikx JP, Matthijsen RA, de Bruine AP, van Bijnen AA, van Dam RM, Dejong CH, and Buurman WA. Human intestinal ischemia-reperfusion-induced inflammation characterized: experiences from a new translational model. The American journal of pathology 176: 2283-2291, 2010.

20. Grootjans J, Thuijls G, Derikx JP, van Dam RM, Dejong CH, and Buurman WA. Rapid lamina propria retraction and zipper-like constriction of the epithelium preserves the epithelial lining in human small intestine exposed to ischaemia-reperfusion. The Journal of pathology 224: 411-419, 2011.

21. Grootjans J, Thuijls G, Verdam F, Derikx JP, Lenaerts K, and Buurman WA. Non-invasive assessment of barrier integrity and function of the human gut. World f Gastrointest Surg 2: 61-69, 2010.

22. Guan Y, Watson AJ, Marchiando AM, Bradford E, Shen L, Turner JR, and Montrose MH. Redistribution of the tight junction protein ZO-1 during physiological shedding of mouse intestinal epithelial cells. American journal of physiology Cell physiology 300: C1404-1414, 2011. 
23. Jakob SM, Parviainen I, Ruokonen E, Hinder R, Uusaro A, and Takala J. Increased splanchnic oxygen extraction because of routine nursing procedures. Critical care medicine 37: 483-489, 2009.

24. Jakob SM, Ruokonen E, Vuolteenaho O, Lampainen E, and Takala J. Splanchnic perfusion during hemodialysis: evidence for marginal tissue perfusion. Critical care medicine 29: 1393-1398, 2001.

25. Knichwitz G, Rotker J, Mollhoff T, Richter KD, and Brussel T. Continuous intramucosal PCO2 measurement allows the early detection of intestinal malperfusion. Critical care medicine 26: 1550-1557, 1998.

26. Krack A, Richartz BM, Gastmann A, Greim K, Lotze U, Anker SD, and Figulla HR. Studies on intragastric $\mathrm{PCO}_{2}$ at rest and during exercise as a marker of intestinal perfusion in patients with chronic heart failure. European journal of heart failure 6: 403-407, 2004.

27. Lambert GP. Intestinal barrier dysfunction, endotoxemia, and gastrointestinal symptoms: the 'canary in the coal mine' during exercise-heat stress? Med Sport Sci 53: 61-73, 2008.

28. Lambert GP, Lang J, Bull A, Pfeifer PC, Eckerson J, Moore G, Lanspa S, and O'Brien J. Fluid restriction during running increases GI permeability. International journal of sports medicine 29: 194-198, 2008.

29. Larson MV, Ahlquist DA, Karlstrom L, and Sarr MG. Intraluminal measurement of enteric mucosal perfusion: relationship to superior mesenteric artery flow during basal and postprandial states in the dog. Surgery 115: 118126, 1994.

30. Lorente JA, Ezpeleta A, Esteban A, Gordo F, de la Cal MA, Diaz C, Arevalo JM, Tejedor C, and Pascual T. Systemic hemodynamics, gastric intramucosal $\mathrm{PCO}_{2}$ changes, and outcome in critically ill burn patients. Critical care medicine 28: 1728-1735, 2000.

31. Lubbers T, Kox M, de Haan JJ, Greve JW, Pompe JC, Ramakers BP, Pickkers P, and Buurman WA. Continuous Administration of Enteral Lipid- and Protein-Rich Nutrition Limits Inflammation in a Human Endotoxemia Model. Critical care medicine 2013.

32. Marchbank T, Davison G, Oakes JR, Ghatei MA, Patterson M, Moyer MP, and Playford RJ. The nutriceutical bovine colostrum truncates the increase in gut permeability caused by heavy exercise in athletes. Am f Physiol Gastrointest Liver Physiol 300: G477-484, 2011.

33. Marchiando AM, Shen L, Graham WV, Edelblum KL, Duckworth CA, Guan Y, Montrose MH, Turner JR, and Watson AJ. The epithelial barrier is maintained by in vivo tight junction expansion during pathologic intestinal epithelial shedding. Gastroenterology 140: 1208-1218 e1201-1202, 2011.

34. Matheson PJ, Wilson MA, and Garrison RN. Regulation of intestinal blood flow. J Surg Res 93: 182-196, 2000.

35. Mensink PB, Moons LM, and Kuipers EJ. Chronic gastrointestinal ischaemia: shifting paradigms. Gut 60: $722-$ $737,2011$.

36. Moses FM. The effect of exercise on the gastrointestinal tract. Sports medicine (Auckland, NZ) 9: 159-172, 1990.

37. Moses FM. Exercise-associated intestinal ischemia. Current sports medicine reports 4: 91-95, 2005.

38. Moses FM. Gastrointestinal bleeding and the athlete. The American journal of gastroenterology 88: 1157-1159, 1993.

39. Nielsen HB, Svendsen LB, Jensen TH, and Secher NH. Exercise-induced gastric mucosal acidosis. Medicine and science in sports and exercise 27: 1003-1006, 1995.

40. Ohri SK, Becket J, Brannan J, Keogh BE, and Taylor KM. Effects of cardiopulmonary bypass on gut blood flow, oxygen utilization, and intramucosal $\mathrm{pH}$. The Annals of thoracic surgery 57: 1193-1199, 1994.

41. Ohri SK, Somasundaram S, Koak Y, Macpherson A, Keogh BE, Taylor KM, Menzies IS, and Bjarnason I. The effect of intestinal hypoperfusion on intestinal absorption and permeability during cardiopulmonary bypass. Gastroenterology 106: 318-323, 1994.

42. Oktedalen O, Lunde OC, Opstad PK, Aabakken L, and Kvernebo K. Changes in the gastrointestinal mucosa after long-distance running. Scand $\mathcal{F}$ Gastroenterol 27: 270-274, 1992.

43. Otte JA, Oostveen E, Geelkerken RH, Groeneveld AB, and Kolkman JJ. Exercise induces gastric ischemia in healthy volunteers: a tonometry study. F Appl Physiol 91: 866-871, 2001.

44. Peters HP, Bos M, Seebregts L, Akkermans LM, van Berge Henegouwen GP, Bol E, Mosterd WL, and de Vries WR. Gastrointestinal symptoms in long-distance runners, cyclists, and triathletes: prevalence, medication, and etiology. The American journal of gastroenterology 94: 1570-1581, 1999.

45. Poeze M, Greve JW, and Ramsay G. Meta-analysis of hemodynamic optimization: relationship to methodological quality. Critical care 9: R771-779, 2005.

46. Rahman SH, Ammori BJ, Holmfield J, Larvin M, and McMahon MJ. Intestinal hypoperfusion contributes to gut barrier failure in severe acute pancreatitis. F Gastrointest Surg 7: 26-35; discussion 35-26, 2003.

47. Rapp-Kesek D, Joachimsson PO, and Karlsson T. Splanchnic blood flow and oxygen consumption: effects of enteral nutrition and dopexamine in the elderly cardiac surgery patient. Acta anaesthesiologica Scandinavica 51: 570-576, 2007.

48. Sandek A, Anker SD, and von Haehling S. The gut and intestinal bacteria in chronic heart failure. Curr Drug Metab 10: 22-28, 2009.

49. Sandek A, Bauditz J, Swidsinski A, Buhner S, Weber-Eibel J, von Haehling S, Schroedl W, Karhausen T, Doehner W, Rauchhaus M, Poole-Wilson P, Volk HD, Lochs H, and Anker SD. Altered intestinal function in patients with chronic heart failure. F Am Coll Cardiol 50: 1561-1569, 2007. 
50. Sawka MN, Young AJ, Francesconi RP, Muza SR, and Pandolf KB. Thermoregulatory and blood responses during exercise at graded hypohydration levels. fournal of applied physiology 59: 1394-1401, 1985.

51. Schwartz N, Hosford M, Sandoval RM, Wagner MC, Atkinson SJ, Bamburg J, and Molitoris BA. Ischemia activates actin depolymerizing factor: role in proximal tubule microvillar actin alterations. The American journal of physiology 276: F544-551, 1999.

52. Shoemaker WC, Appel PL, Kram HB, Waxman K, and Lee TS. Prospective trial of supranormal values of survivors as therapeutic goals in high-risk surgical patients. Chest 94: 1176-1186, 1988.

53. Ter Steege RW, Geelkerken RH, Huisman AB, and Kolkman JJ. Abdominal symptoms during physical exercise and the role of gastrointestinal ischaemia: a study in 12 symptomatic athletes. Br f Sports Med 2011.

54. Thuijls G, de Haan JJ, Derikx JP, Daissormont I, Hadfoune M, Heineman E, and Buurman WA. Intestinal cytoskeleton degradation precedes tight junction loss following hemorrhagic shock. Shock 31: 164-169, 2009.

55. Thuijls G, Derikx JP, de Haan JJ, Grootjans J, de Bruine A, Masclee AA, Heineman E, and Buurman WA. Urinebased detection of intestinal tight junction loss. Fournal of clinical gastroenterology 44: e14-19, 2010.

56. Thuijls G, Derikx JP, de Kruijf M, van Waardenburg DA, van Bijnen AA, Ambergen T, van Rhijn LW, Willigers HM, and Buurman WA. Preventing enterocyte damage by maintenance of mean arterial pressure during major nonabdominal surgery in children. Shock 37: 22-27, 2012.

57. van Haren FM, Rozendaal FW, and van der Hoeven JG. The effect of vasopressin on gastric perfusion in catecholamine-dependent patients in septic shock. Chest 124: 2256-2260, 2003.

58. van Haren FM, Sleigh JW, Pickkers P, and Van der Hoeven JG. Gastrointestinal perfusion in septic shock. Anaesth Intensive Care 35: 679-694, 2007.

59. van Nieuwenhoven MA, Brouns F, and Brummer RJ. The effect of physical exercise on parameters of gastrointestinal function. Neurogastroenterol Motil 11: 431-439, 1999.

60. van Nieuwenhoven MA, Brouns F, and Brummer RJ. Gastrointestinal profile of symptomatic athletes at rest and during physical exercise. European journal of applied physiology 91: 429-434, 2004.

61. Vermeulen Windsant IC, Hellenthal FA, Derikx JP, Prins MH, Buurman WA, Jacobs MJ, and Schurink GW. Circulating intestinal fatty acid-binding protein as an early marker of intestinal necrosis after aortic surgery: a prospective observational cohort study. Annals of surgery 255: 796-803, 2012.

62. von Duvillard SP, Arciero PJ, Tietjen-Smith T, and Alford K. Sports drinks, exercise training, and competition. Current sports medicine reports 7: 202-208, 2008.

63. Vreugdenhil AC, Wolters VM, Adriaanse MP, Van den Neucker AM, van Bijnen AA, Houwen R, and Buurman WA. Additional value of serum I-FABP levels for evaluating celiac disease activity in children. Scandinavian journal of gastroenterology 46: 1435-1441, 2011.

64. Wright $\mathrm{H}$, Collins M,Villiers RD, and Schwellnus MP. Are splanchnic hemodynamics related to the development of gastrointestinal symptoms in ironman triathletes? A prospective cohort study. Clin f Sport Med 21: 337-343, 2011.

65. Zacho HD, Kristensen NB, Henriksen JH, and Abrahamsen J. Validation of (9) (9)mTechnetium-labeled mebrofenin hepatic extraction method to quantify meal-induced splanchnic blood flow responses using a porcine model. Fournal of applied physiology 112: 877-882, 2012. 
Gastrointestinal (GI) integrity is essential to preserve the two key functions of the gut, i.e. the absorption of dietary nutrients and water and the maintenance of an effective barrier against harmful luminal compounds. Chapter 1 outlines the anatomy and regulation of the GI epithelial barrier, including a brief overview of situations associated with GI barrier dysfunction. The anatomical architecture of the epithelial barrier mainly builds on small intestinal epithelial cells and junctional complexes between these cells. Rapid renewal of the epithelium along the small intestinal crypt-villus axis contributes to the remarkable ability of the intestinal epithelium to adapt to physiological and pathophysiological stimuli such as non-steroid anti-inflammatory drug (NSAID) consumption and strenuous physical exercise (chapter 2).

Intestinal epithelial integrity loss can be established by detection of components of the epithelial barrier in blood or urine, detection of circulating (anti)bacterial products or by permeability analysis. Part II of this thesis focused on markers for enterocyte injury and permeability analysis. Enterocyte injury was measured by detection of fatty acid binding proteins (FABP). Data of previous human studies revealed that intestinal (I)-FABP and liver (L)-FABP are expressed in the mucosa of the small intestine, especially the jejunum, while ileal-bile acid binding protein (I-BABP) is specifically expressed in the ileum. Additionally, it was shown that especially the mature enterocytes at the top of the intestinal villi contain I-FABP. Since these proteins are rapidly released upon cellular injury, they are established as useful, early markers of intestinal integrity loss. The use of I-FABP, L-FABP and I-BABP for diagnosis of intestinal ischemia with ischemia-induced intestinal injury was studied in chapter $\mathbf{3}$ of this thesis. All three markers were found to be increased in urine of patients with intestinal ischemia compared to patients with other final diagnoses, and plasma I-FABP and L-FABP levels were also higher in case of intestinal ischemia. Detection of these proteins above their cut-off point increased post-test probability for intestinal ischemia, strongly suggesting that detection of I-FABP, L-FABP and I-BABP can improve early diagnosis of intestinal ischemia.

Permeability analysis is an additional, more functional measure of GI epithelial barrier integrity. Detection of orally ingested permeability probes with different molecular weights in urine or plasma reflects the permeability of the GI mucosa. Many permeability tests are available, but currently no consensus exists. An important aim of this thesis was to compare probe excretion and accuracy of different permeability tests and to determine whether one of the tested assays could be recommended above the others. Chapter 4 describes the comparison of two permeability tests in a randomized controlled trial: The first is based on the urinary excretion of three differently sized polyethylene glycol (PEG) probes, while the dual sugar test uses $5 \mathrm{~g}$ of lactulose and $0.5 \mathrm{~g}$ of rhamnose to evaluate small intestinal permeability. Healthy individuals received two oral doses of NSAID indomethacin, a drug known to increase GI permeability. Loss of barrier integrity was confirmed by significantly increased plasma I-FABP levels in the indomethacin-challenged state compared to placebo. Urinary excretion of PEG and sugars increased after indomethacin. Urinary excretion data and diagnostic accuracy of the tests showed 


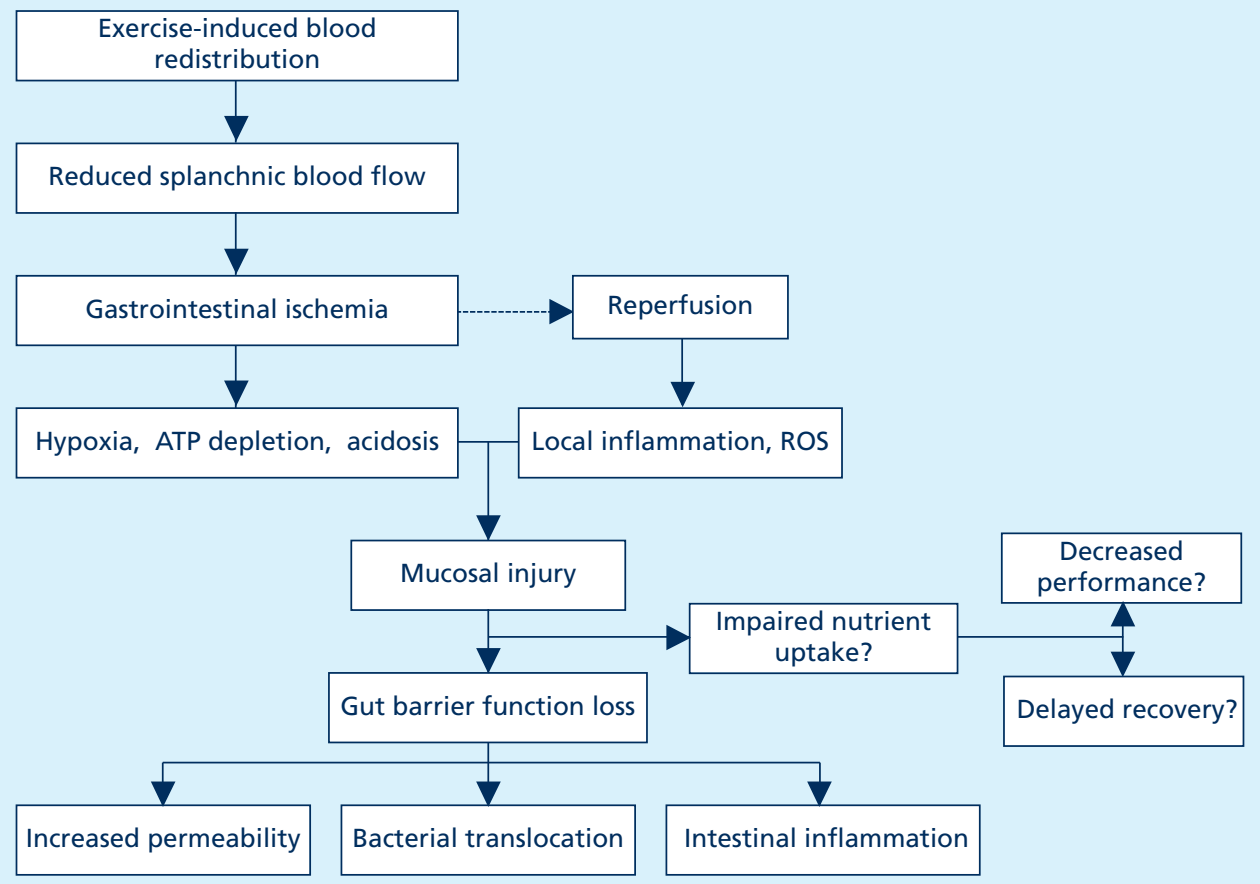

Figure 1: Schematic overview of the processes proposed to play a role in the development of exercise-induced gastrointestinal injury

Strenuous physical exercise leads to redistribution of blood, shunting blood away from the splanchnic area, thereby significantly reducing splanchnic blood flow. The ensuing ischemia leads to tissue hypoxia, local depletion of adenosinetriphosphate (ATP), and acidosis. After exercise, when splanchnic circulation is restored, reperfusion injury may develop, characterized by local inflammation and formation of reactive oxygen species (ROS). Both ischemia and reperfusion sequelae lead to mucosal damage and gut wall integrity loss, which is accompanied by increased permeability, bacterial translocation and intestinal inflammation.

It remains to be clarified whether the exercise-induced mucosal injury impairs nutrient uptake, potentially decreasing athletic performance and delaying post-exercise recovery.

high similarity for detection of indomethacin-induced increases in gastrointestinal permeability. These tests do not allow site-specific GI permeability analysis and require the oral intake of relatively large probe doses, thereby possibly affecting epithelial homeostasis. A novel multi-sugar test was developed to allow site-specific assessment in a more physiological setting, i.e. with a reduced oral probe load. In chapter 5 and $\mathbf{6}$, the improved analytical work-up and validation of this multi-sugar test are reported. The multi-sugar assay contains sucrose for gastroduodenal permeability, lactulose and rhamnose to reflect small intestinal permeability, and sucralose and erythritol for large intestinal permeability. Probes were first separated using liquid chromatography (LC), after which mass spectrometry (MS) was used for final detection. This LC-MS approach resulted in a considerably decreased limit of detection, allowing a 5-fold reduction of 
the oral lactulose dose. The novel multi-sugar test was tested against the classical dual sugar test in a randomized crossover study in chapter 6. Urinary sucrose excretion and the sucrose/rhamnose ratio increased after indomethacin intake compared to placebo, suggesting increased gastroduodenal permeability determined by the multi-sugar test. Increased small intestinal permeability was detected by both tests after indomethacin intake, as reflected by elevated lactulose/rhamnose (L/R) ratios compared to placebo. However, individual probe analysis suggested that the high lactulose dose of the dual sugar test decreases monosaccharide permeability. Large intestinal permeability was unchanged after indomethacin. Altogether, the multi-sugar test was found to provide a physiological test condition for site-specific whole-gut permeability analysis. While clinical validation studies are essential to determine the usefulness of the multisugar test in different patient groups, the comparative studies described in this thesis improved our knowledge of GI permeability analysis in man.

Part III of this thesis focused on exercise-induced splanchnic hypoperfusion and GI integrity loss, and a comprehensive overview of these phenomena was given in chapter 2 of this thesis, summarized in Figure 1.

In short, heavy physical exercise leads to redistribution of the GI blood flow in favor of active muscle, heart and skin, thereby significantly reducing perfusion in the splanchnic area. This phenomenon was studied and described in chapter 7 using gastric air tonometry. During one hour of intensive cycling, splanchnic hypoperfusion rapidly developed and continued to exist in the post-exercise recovery period. Intestinal ischemia leads to cellular hypoxia, energy depletion and tissue acidosis, ultimately leading to small intestinal injury, reflected by significantly elevated plasma I-FABP levels. The latter significantly correlated with loss of GI barrier integrity, reflected by increased plasma L/R ratios. Additionally, intestinal damage may impair nutrient uptake, which may in turn affect physical performance and post-exercise recovery. In chapter $\mathbf{8}$, this hypothesis was studied. In line with the results in chapter 7, exercise induced small intestinal injury in healthy young men, and this intestinal injury correlated negatively with in vivo rates of protein digestion and absorption, when proteins are consumed directly post-exercise. These data imply that exercise attenuates protein digestion and absorption kinetics during acute post-exercise recovery compared to resting conditions due to enterocyte damage. These findings suggest an additional challenge for professional athletes and patients with chronic diseases, since especially these populations benefit from nutritional stimulation of early and complete recovery after physical exertion. While from chapter 7 and 8 it is clear that exercise itself induces significant small intestinal injury and compromises GI functioning, the intake of the NSAID ibuprofen prior to exercise was found to significantly worsen exercise-induced intestinal injury and increase GI permeability (chapter 9). These data are important, since many athletes use NSAIDs on a regular basis to prevent anticipated pain without being aware of the adverse effects of these drugs. Our findings in combination with the alarming indications that NSAIDs may induce cardiac 
events strongly discourage the use of NSAIDs, especially for non-existing pain. In chapter 10, oral supplementation of L-citrulline prior to exercise was shown to improve splanchnic perfusion during exercise in healthy athletes compared to placebo amino acid L-alanine. Consequently, enterocyte damage was attenuated during exercise in case of pre-exercise L-citrulline supplementation, while parameters of cardiac function and performance did not change. These results suggest L-citrulline administration to be a promising therapy for improvement of splanchnic blood flow in case of hypoperfusion. 


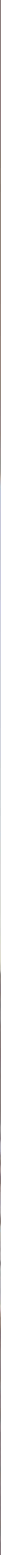

Chapter 13 
Een intacte darmbarrière is van essentieel belang om de twee belangrijkste functies van de darm te behouden. Deze functies zijn (1) opname van voedingsstoffen en water en (2) preventie van het binnendringen van potentieel schadelijke stoffen die zich in het darmkanaal bevinden, zoals bacteriële producten en verteringssappen. Hoofdstuk 1 schetst de anatomie en de regulatie van de darmbarrière, inclusief een kort overzicht van enkele situaties waarin sprake is van darmbarrièreverlies. Belangrijke onderdelen van de darmbarrière zijn de darmepitheelcellen en de verbindende complexen tussen deze cellen. Het darmepitheel wordt volledig vernieuwt in 3-4 dagen. Deze snelle vernieuwing draagt bij aan het opmerkelijke vermogen van de darm om zich aan te passen aan veranderende omstandigheden, zoals het gebruik van 'non-steroid antiinlammatory drugs' (NSAIDs) en zware fysieke inspanning (hoofdstuk 2).

Darmbarrièreverlies kan worden vastgesteld door het detecteren van componenten van deze barrière in bloed of urine, door detectie van (anti)bacteriële producten in de bloedbaan, of door analyse van de doorlaatbaarheid van de darmwand, ook wel darmpermeabiliteit genoemd.

Deel II van dit proefschrift richt zich op markers voor darmcelschade en op het testen van de darmpermeabiliteit. Darmcelschade werd gemeten middels 'intestinal' en 'liver fatty acid binding proteins' (I-FABP en L-FABP, respectievelijk), en 'ileal bile acid binding protein' (I-BABP), kleine eiwitten die zich bevinden in het epitheel van de dunne darm. Eerdere studies toonden aan dat met name de volgroeide darmcellen op de toppen van de darmvilli deze eiwitten bevatten, en dat deze snel worden afgegeven wanneer darmcelschade optreedt. Hierdoor zijn deze specifieke eiwitten goed bruikbaar als vroege herkenners van darmcelschade. Het gebruik van I-FABP, L-FABP en I-BABP voor de diagnose van darmischemie en door ischemie ontstane darmschade werd bestudeerd in hoofdstuk 3 van dit proefschrift. Darmischemie is een aandoening waarbij er geen of te weinig bloed naar de darm gaat, waardoor darmschade optreedt. Alle drie de markers bleken verhoogd aanwezig te zijn in de urine van patiënten met darmischemie, vergeleken met patiënten met andere diagnoses. Daarnaast waren de bloedwaarden voor I-FABP en L-FABP ook hoger in geval van darmischemie. Wanneer de bloed- of urinewaarden van deze eiwitten boven hun drempelwaarde uitstegen, bleek er sprake te zijn van een verhoogde kans op darmischemie bij deze patiënten. Deze bevindingen suggereren dat de detectie van I-FABP, L-FABP en I-BABP kan bijdragen aan het vroeg vaststellen van darmischemie in patiënten.

Permeabiliteitstesten zijn een andere manier om informatie te krijgen over de darmbarrière. De proefpersoon of patiënt drinkt een oplossing met teststoffen van verschillende grootte, waarna de concentraties van deze stoffen in bloed of urine bepaald wordt als weerspiegeling van de doorlaatbaarheid van de darmwand. Er zijn veel verschillende tests beschikbaar, maar er is geen consensus over welke test gebruikt moet worden. Een belangrijk doel van dit proefschrift was om de uitkomsten en de nauwkeurigheid van de verschillende permeabiliteitstesten te vergelijken en om te bepalen of een van de onderzochte tests kon worden aanbevolen boven de anderen.

Hoofdstuk 4 beschrijft de vergelijking van twee permeabiliteittesten, elk bestaande uit verschillende teststoffen: De eerste bestaat uit drie verschillende polyethyleenglycol-polymeren 
(PEG); de andere, klassieke test bestaat uit $5 \mathrm{~g}$ lactulose en $0,5 \mathrm{~g}$ rhamnose, allebei suikers. Voor het onderzoek ontvingen de proefpersonen tweemaal indometacine, een NSAID waarvan bekend is dat het de doorlaatbaarheid van de darmwand tijdelijk verhoogt. Dit laatste werd bevestigd door een evidente stijging van I-FABP na inname van indomethacine in vergelijking met placebo. PEG en suikerwaarden in urine namen beiden toe na inname van indomethacine. Beide testen bleken veel gelijkenis te vertonen, zowel in de testuitkomsten als in de diagnostische nauwkeurigheid van de testen voor de detectie van door indomethacine veroorzaakte toegenomen darmpermeabiliteit. Helaas kan de permeabiliteit van specifieke delen van de darm niet bepaald worden met deze testen. Bovendien dient de proefpersoon relatief grote hoeveelheden van de teststof in te nemen, waardoor mogelijk het natuurlijk evenwicht in de darm verstoord wordt. Zodoende werd er een nieuwe multi-suikertest ontwikkeld om wel specifieke informatie te kunnen verkrijgen en waarbij kleinere hoeveelheden teststof ingenomen hoeft te worden.

In hoofdstuk 5 en 6 van dit proefschrift werden de verbeterde detectiemethode en de validatie van deze multi-suikertest beschreven. De multi-suikertest bevat sucrose voor bepaling van de permeabiliteit van de maag en twaalfvingerige darm, lactulose en rhamnose voor de dunne darmpermeabiliteit, en sucralose en erytritol voor de dikke darmpermeabiliteit. Voor detectie van de suikers wordt er eerst gebruik gemaakt van vloeistofchromatografie (LC), waarna middels massaspectrometrie (MS) de suikergehaltes in bloed en urine worden bepaald. Deze LC-MS aanpak verlaagde de minimale detectiegrens aanzienlijk, waardoor het mogelijk werd om lagere suikerconcentraties accuraat te meten en bovendien een 5-voudige reductie van de in te nemen dosis lactulose mogelijk was. De nieuwe multi-suikertest werd getoetst aan de klassieke suikertest in een gerandomiseerde cross-over studie beschreven in hoofdstuk 6. Zowel sucrose als de sucrose/rhamnose ratio in urine steeg na inname van indomethacine in vergelijking met de placebo, wat erop wijst dat er sprake was van een verhoogde gastroduodenale permeabiliteit na indomethacine inname, gemeten middels de multi-suikertest. Verhoogde dunne darmpermeabiliteit werd gevonden na indomethacine inname met beide tests, zoals weerspiegeld door hogere lactulose/rhamnose (L/R) ratio's, vergeleken met placebo. Echter, de resultaten suggereerden ook dat de hoge dosis lactulose in de klassieke suikertest ervoor zorgt dat er minder rhamnose wordt uitgescheiden, wat betekent dat de testsubstanties zelf de resultaten kunnen beïnvloeden. Concluderend gaven de studieresultaten aan dat de multi-suikertest een geschikte en vooral betrouwbare test is om de permeabiliteit van het gehele maagdarmstelsel te beoordelen. Hoewel klinische validatiestudies essentieel zijn om het nut van de multi-suikertest in verschillende patiëntengroepen te bepalen, zijn de studies beschreven in dit proefschrift belangrijk omdat zij onze kennis van de darmpermeabiliteitsanalyses in de mens vergroten.

Deel III van dit proefschrift heeft betrekking op de verminderde doorbloeding van het maagdarmstelsel en de daaropvolgende darmbarrièreverlies die optreden tijdens intensieve lichame lijke inspanning. Een uitgebreid overzicht van deze fenomenen werd gegeven in hoofdstuk 2 van dit proefschrift. Kort samengevat leidt zware lichamelijke inspanning tot een herverdeling 
van de bloedvoorziening. Het maagdarmstelsel wordt tijdelijk minder goed doorbloed, zodat de actieve spieren, het hart en de huid meer bloed kunnen ontvangen. De kritieke vermindering van de doorbloeding van het maagdarmstelsel wordt hypoperfusie genoemd, en is onderzocht in de studie beschreven in hoofdstuk 7. Gedurende een uur intensief fietsen, ontwikkelde de hypoperfusie in het maagdarmstelsel zich snel, en bleef bestaan in de herstelperiode na het sporten. Hypoperfusie kan leiden tot een tekort aan zuurstof en energie in de darmcellen, waardoor verzuring van het weefsel en uiteindelijk dunne darmschade ontstaan, weerspiegeld door verhoogde I-FABP-waarden in het bloed. Deze darmschade gaf aanzet tot darmbarrièreverlies, weerspiegeld door verhoogde L/R ratio's ten opzichte van rust. Darmschade kan de opname van voedingsstoffen bemoeilijken, waardoor mogelijk ook de fysieke prestaties en hersteltijd na het sporten negatief beïnvloed worden.

In hoofdstuk 8 werd deze hypothese onderzocht. In lijn met hoofdstuk 7, leidde intensieve inspanning tot darmschade bij gezonde jonge mannen, en deze darmschade had een negatief effect op de vertering en absorptie van eiwitten die de atleten innamen direct na de fysieke inspanning. Met andere woorden, inspanning vermindert de verterings- en absorptiecapaciteit voor eiwit in de herstelperiode na inspanning vergeleken met rust. Deze bevindingen suggereren een extra uitdaging voor professionele atleten en patiënten met chronische ziekten, omdat vooral bij deze mensen voeding een belangrijk middel is om het herstel na de fysieke inspanning te bevorderen.

Uit hoofdstuk 7 en $\mathbf{8}$ is reeds gebleken dat inspanning dunne darmschade en verminderde darmfunctie veroorzaakt.

De resultaten in hoofdstuk 9 lieten zien dat de inname van de NSAID ibuprofen vóór inspanning deze darmschade en darmbarrièreverlies aanzienlijk verergert. Deze gegevens zijn van belang, omdat veel atleten regelmatig NSAIDs gebruiken in de hoop geanticipeerde pijn te voorkomen, zonder dat daar bewijs voor is en zonder zich bewust te zijn van de nadelige effecten van deze geneesmiddelen. Onze bevindingen in combinatie met de alarmerende aanwijzingen dat NSAID's hartproblemen kunnen veroorzaken, ontraden het gebruik van NSAID's sterk, indien hier geen medisch aanwijzing voor is.

Ten slotte trachten we in de crossover studie beschreven in hoofdstuk 10 middels een specifieke voedingsinterventie voorafgaand aan de inspanning de doorbloeding van het maagdarm-stelsel tijdens en na inspanning te verbeteren en de inspannings-gerelateerde darmschade te reduceren of zelfs te voorkomen. Inderdaad toonden de resultaten van deze studie aan dat hypoperfusie tijdens inspanning kon worden voorkomen door voorafgaand aan de inspanning L-citrulline te consumeren, terwijl inname van placebo aminozuur L-alanine dit effect niet had. Het behoud van de circulatie in het maagdarmstelsel tijdens inspanning zorgde ervoor dat ook de integriteit van het darmepitheel minder verstoord was, terwijl de parameters van de hartfunctie en de inspanningsprestatie niet verslechterden. Deze studieresultaten suggereren dat L-citrulline inname een veelbelovend middel is voor verbetering van de circulatie in geval van hypoperfusie van het maagdarmstelsel. 


\section{DANKWOORD}

Graag wil ik aan het einde van mijn promotietraject met trots terugkijken op wat we samen bereikt hebben.

Prof. Buurman, beste Wim, ik wil je graag bedanken voor de mogelijkheden en de begeleiding die je me door de jaren geboden hebt. We moesten wat op elkaar ingespeeld raken, maar toen dat voor elkaar was, liep het ook goed. Je veelvuldige pogingen om mij woordelijk uit te dagen hebben me geholpen om altijd mijn stappen te kunnen onderbouwen en mijn eigen grenzen aan te geven. Dank voor je tijd, je gekkigheden en je vertrouwen!

Prof. Dejong, beste Kees, ik wil je hartelijk danken voor je vertrouwen en de mogelijkheden die je me hebt aangereikt, allereerst bij het maken van dit proefschrift en vervolgens bij de vlotte inlijving in het team van de heelkunde. Jouw enthousiasme en interesse voor het onderzoek, voor mij en mijn sportprestaties heb ik altijd erg gewaardeerd. Ik hoop nog veel van je te mogen leren!

Beste Kaatje! Dank voor de vele uurtjes overleggen, manuscripten doorlezen, en nóg eens kritisch naar de stukken, de referenties en de figuren te kijken! Je hebt een engelengeduld! Ook heel veel dank voor je hulp bij de perikelen onderweg. Het is klaar!

Prof. Stassen, beste Laurents, toen ik eerst als Kim Holland en later gewoon als Kim van Wijck met je sprak over mijn voornemen om na mijn promotie-onderzoek het team van de heelkunde aan te sterken, was jij meteen enthousiast. Ik wil je graag bedanken voor dat enthousiasme en voor het vertrouwen dat ik kreeg om zo vlot aan de slag te mogen in de kliniek. Ik hoop nog veel van je te mogen leren; binnenkort een eerste laparoscopische ingreep samen?

Dr. Hoofwijk, beste Ton. Bedankt voor je steun en vertrouwen in de Blitzkrieg van onderzoeker tot clinicus. Je wijze woorden over het vak en het leven daaromheen heb ik graag ter harte genomen. Ik kom met plezier terug naar het Orbis na mijn eerste jaren in het Maastrichtse, en dan gaan we er iets moois van maken!

Prof. Van Loon, beste Luc. Onderzoeker en sportman in hart en spieren. Dank voor je zeer toegankelijke hulp bij onze projecten samen! De snelheid en secuurheid waarmee jij de manuscripten doornam, was elke keer weer verademend. $\mathrm{Nu}$ ik weer in het Maastrichtse verblijf, misschien binnenkort een keer samen zitten om te brainstormen over mooie vervolgplannen? 
Prof. Soeters, beste Peter. Toen wij u benaderden en vroegen om op te treden als voorzitter van de leescommissie zei u direct ja. Hartelijk dank voor uw bereidwilligheid om deze taak te vervullen en voor het flitsend optreden om de antwoorden van alle commissieleden tijdelijk te verkrijgen. Ik ben vereerd dat $\mathrm{u}$ aanwezig bent bij de oppositie.

Ook de overige leden van de corona en van de leescommissie wil ik graag hartelijk danken voor de tijd en de interesse voor het lezen en beoordelen van dit proefschrift.

De studies beschreven in dit proefschrift zijn gedaan binnen het framework van Top Institute Food and Nutrition (TIFN). Ik wil met name graag Jan Dekker en de leden van de Maastrichtse TIFN (MDL) groep graag bedanken voor de mogelijkheden die mij geboden zijn en voor de brainstormsessies op de woensdagochtenden.

Prof. Kolkman, beste Jeroen. Dank voor je inwijding in de wondere wereld van de tonometrie zo'n 5 jaar geleden. Toen ik vroeg of ik de kunst eens af zou mogen komen kijken, nodigde je me meteen uit in Twente waarbij je alle tijd nam om me de kunsten van de darmischemie diagnostiek te laten zien. Dank voor je enthousiasme en voor je tijd bij het lezen en beoordelen van mijn promotieboekje. Ik zie ernaar uit met je van gedachten te wisselen!

Dr. Poeze, beste Martijn. Ook jou wil ik graag bedanken voor je introductie in de tonometrie en voor de samenwerking over de jaren om toch samen een aantal mooie artikelen te publiceren. $\mathrm{Nu}$ die ene laatste nog!

Beste Nina, partner in de citrulline crime, dank voor je hulp bij het review en voor al je werk voor ons artikel. Ik hoop dat je snel ook op de promotie-sneltrein kan stappen, want dat heb je na de doorgemaakte tegenspoed wel verdiend!

Naast bovenstaande personen zijn er natuurlijk nog veel meer mensen die bijgedragen hebben aan dit promotiewerk. Graag wil ik al mijn collega's in het onderzoek en in de kliniek, zowel in Sittard als in Maastricht, hartelijk danken voor de gezelligheid en steun! En iets minder voor het moment waarop ik weer eens in de papierbak belandde.

Graag wil ik bij naam noemen Annemarie, de ELISA-koningin; Bas, dan minstens prins van de ELISA en natuurlijk proefpersoon ten top; Babs, PEG-liefhebber, en Hans - dank voor alle analyses die jullie met zoveel precisie hebben uitgevoerd! Ook dank aan alle student-onderzoekers die hun bijdrage hebben geleverd aan de tot standkoming van dit proefschrift, met name Thomas, Nele, Carola, Nadine, Ilja, Esther en Ben. 
Paula, hartelijk dank voor je enorme hulp bij de layout van boekje en website! Er gingen geen drie dagen voorbij of ik had alweer een nieuwe mail terug, zodat ik in mijn nachtelijke uurtjes altijd iets te doen had. Het ziet er super uit!

Proefpersonen! Veel dank ben ik jullie verschuldigd, want zonder proefpersonen die zich voor mij in het zweet wilden werken, waren we mooi aan de kade blijven liggen. Gelukkig is een kinderhand al snel gevuld, en waren we na wat dikbelegde boterhammen al snel weer on speaking terms. Natuurlijk wilden jullie over een week wel weer die tonometriekatheter in de neus. Heren, en dan vooral proefpersonen van het eerste uur, Bas en Kostan, heel veel dank voor al jullie inzet en het doortrappen als ik jullie naar de denkbeeldige finish stond te schreeuwen. Wees gerust, het MEC protocol van deze studie is afgesloten.

Vrienden en familie, hopelijk ben ik er door de jaren heen in geslaagd om duidelijk te maken hoeveel jullie voor mij betekenen. Met jullie om mij heen, life is good! 


\section{CURRICULUM VITAE EN PUBLICATIES}

Kim van Wijck was born on June 18th 1984 in Ouderkerk aan de Amstel, the Netherlands. She started high school at the Keizer Karel College (Gymnasium) in Amstelveen. After her graduation in 2002, she started her medical training at the Maastricht University Medical Center (MUMC+) from September 2002 to July 2008. After being elected with an international scholarship by SWG/LAD, she did a prolonged internship of Pediatrics and Pediatric Surgery at the University Hospital of Pretoria, South Africa. December 2006 until January 2007, Kim did a short internship at the Wilhemina Children's hospital in Utrecht at the Department of Pediatric Surgery (D van der Zee, MD PhD). During her senior year, Kim combined a clinical and a research elective at the Department of Surgery and Pediatric Surgery at MUMC+, leading to oral presentations at national and international conferences and publishment of data in scientific journals. In July 2008, Kim started her PhD project at the Department of Surgery at MUMC+ (WA Buurman, MD PhD and CHC Dejong, MD PhD) for Top Institute Food and Nutrition. Multiple studies were performed, of which data have been presented and published both nationally and internationally. During her years as $\mathrm{PhD}$ student, Kim worked as a medical volunteer for Service Médical at multiple sports events, and she was personally active as a triathlete competing at national and international races. In July 2012, the scientific work described in this thesis was completed and Kim started working at the Surgical Department of Orbis Medical Center in Sittard (TG Hoofwijk, MD PhD), the Netherlands, and in January 2013 she was accepted as surgical trainee, and continued her career at MUMC+ (LPS Stassen, MD PhD). 


\section{SCIENTIFIC OUTPUT}

van Wijck K, Lenaerts K, Grootjans J, Wijnands KA, Poeze M, van Loon LJ, Dejong CH, Buurman WA. Physiology and pathophysiology of splanchnic hypoperfusion and intestinal injury during exercise: strategies for evaluation and prevention. Am J Physiol Gastrointest Liver Physiol 2012; 303:G155-68.

Adriaanse MP, Tack GJ, Passos VL, Damoiseaux JG, Schreurs MW, van Wijck K, Riedl RG, Masclee AA, Buurman WA, Mulder CJ, Vreugdenhil AC. Serum I-FABP as marker for enterocyte damage in coeliac disease and its relation to villous atrophy and circulating autoantibodies. Aliment Pharmacol Ther 2013;37:482-490.

van Wijck K, Pennings B, van Bijnen AA, Senden JM, Buurman WA, Dejong CH, van Loon LJ, Lenaerts K. Dietary protein digesion and absorption are impaired during acute post-exercise recovery in young men. Am J Physiol Regul Integr Comp Physiol 2013;304:R356-361.

van Wijck K, Bessems BA, van Eijk HM, Buurman WA, Dejong CH, Lenaerts K. Polyethylene glycol versus dual sugar assay for gastrointestinal permeability analysis: is it time to choose? Clin Exp Gastroenterol 2012;5:139-150.

van Wijck K, Lenaerts K, van Bijnen AA, Boonen B, van Loon LJ, Dejong CH, Buurman WA. Aggravation of exercise-induced intestinal injury by ibuprofen in athletes. Med Sci Sports Exerc 2012;44:2257-2262.

Highlighted in The New York Times (online version, December 5th, 2012 and printed version, December 12th, 2012), Medscape alert issue December 2012 and several sports magazines.

van Wijck K, Buurman WA. Ischemia-Reperfusion Injury (chapter). Encyclopedia of Exercise Medicine in Health and Disease. 2012. DOI 10.1007/978-3-540-29807-6.

van Wijck K, Verlinden TJM, van Eijk HMH, Dekker J, Buurman WA, Dejong CHC, Lenaerts K. Novel multi-sugar assay for site-specific gastrointestinal permeability analysis: a randomized controlled crossover trial. Clin Nutr 2013;32:245-251.

van Wijck K, van Eijk HM, Buurman WA, Dejong CH, Lenaerts K. Novel analytical approach to a multi-sugar whole gut permeability assay. J Chromatogr B AnalytTechnol Biomed Life Sci 2011;879:2794-801.

van Wijck K, Lenaerts K, van Loon LJ, Peters WH, Buurman WA, Dejong CH. Exercise-induced splanchnic hypoperfusion results in gut dysfunction in healthy men. PLoS ONE 2011;6:e22366. 
Thuijls G, van Wijck K, Grootjans J, Derikx JP, van Bijnen AA, Heineman E, Dejong CH, Buurman WA, Poeze M. Early diagnosis of intestinal ischemia using urinary and plasma fatty acid binding proteins. Ann Surg 2011;253:303-8.

Thuijls G, Derikx JP, van Wijck K, Zimmerman LJ, Degraeuwe PL, Mulder TL, van der Zee DC, Brouwers HA, Verhoeven BH, van Heurn LW, Kramer BW, Buurman WA, Heineman E. Non-invasive markers for early diagnosis and determination of the severity of necrotizing enterocolitis. Ann Surg 2010;251:1174-80.

van Wijck K, de Jong JR, van Heurn LW, van der Zee DC. Prolonged antibiotic treatment does not prevent intra-abdominal abscesses in perforated appendicitis. World J Surg 2010;34: 3049-53.

\section{Scientific presentations}

van Wijck K, Verlinden TJM, Dekker J, Buurman WA, Dejong CH, Lenaerts K. Multi-sugar permeability test: a promising new tool for accurate gut permeability assessment in health and disease UEGW 2011, Stockholm, Sweden; DDW 2011, Chicago, USA. Published in Gastroenterology 2011;140(5): S-88.

van Wijck K, Lenaerts K, van Loon LJ, Senden JM, Buurman W, Dejong CH. Increased small intestinal permeability and liver damage after exhaustive physical exercise in healthy individuals (poster of distinction) DDW 2011, Chicago, USA. Gastroenterology 2011;140(5): S-647. Medical Postgraduate Conference 2010, Hradec Kralove, Czech.

van Wijck K, Lenaerts K, van Loon LJ, Senden JM, Dejong CH, Buurman W. Functional splanchnic hypoperfusion precedes intestinal epithelial cell damage during strenuous exercise (oral) NVGE 2010, Veldhoven, Netherlands; (oral) DDW 2010, New Orleans, USA. Gastroenterology 2010;138(5): S-72.

van Wijck K, Lenaerts K, van Loon LJC, Buurman WA, Dejong CHC. Exercise induces splanchnic hypoperfusion in healthy men. (oral) MedicalPostgraduate Conference 2010, HradecKralove, Czech.

van Wijck K, de Jong JR, van Heurn LW, van der Zee DC. Antibiotics based on CRP does not reduce abscess rate in children operated for perforated appendicitis. EurCongress of PaediatricSurgery 2009, Graz, Austria.

van Wijck K, Thuijls GT, van Heurn LW. Non-invasive detection of gut wall inflammation in neonates suspected for necrotizing enterocolitis. (oral) Chirurgendagen 2008, Veldhoven, Netherlands; (oral) European Congress of Paediatric Surgery 2008, Istanbul, Turkey. 


\section{GRANDS AND AWARDS}

2012 Award for novel study of broad interest to sports physiology - Highlighted commentary Sports Medicine Bulletin (SMB).

2011 Poster of distinction Digestive Disease Week 2011, Chicago, USA.

2011 Best of Digestive Disease Week session held by dr. PR Holt at UEGW 2011, Stockholm, Sweden.

2011 ASNEMGE Young Investigators Initiative 2011, Stockholm, Sweden.

2009 Travel Grant Duch Gastroenterology Federation (NVGE).

2007 Travel award Stichting Werkgelegenheid Geneeskundigen/Landelijke vereniging Artsen in Dienstverband Subsidie 